FWS SSR No.2337

Copy 1

\author{
- \\ THW COPY \\ DDFDOFNOF \\ NON-CIRC. \\ OCEANOGRAPHIC OBSERVATIONS, \\ 1956, EAST COAST \\ OF THE UNITED STATES
}

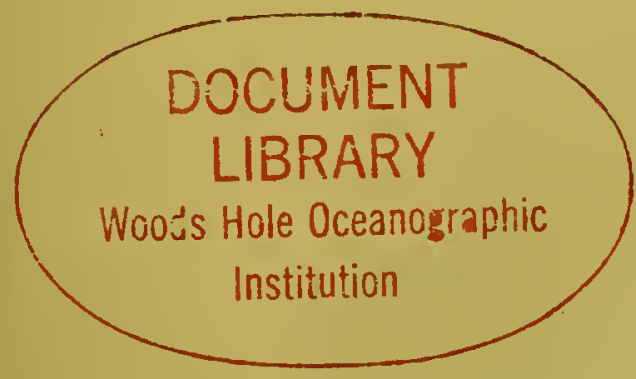

SPECIAL SCIENTIFIC REPORT-FISHERIES NNo. 233
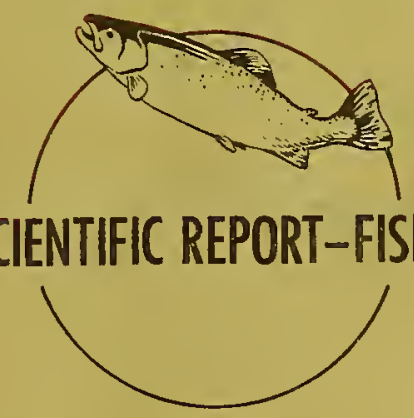


\section{EXPLANATORY NOTE}

The series embodies results of investigations, usually of restricted scope, intended to aid or direct management or utilization practices and as guides for administrative or legislative action. It is issued in limited quantities for official use of Federal, State or cooperating agencies and in processed form for economy and to avoid delay in publication.

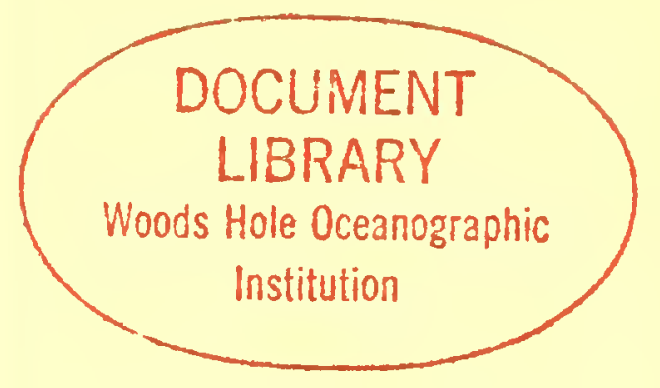


United States Department of the Interior, Fred A. Seaton, Secretary U. S. Fish and Wildifife Service

OCEANOGRAPHIC OBSERVATIONS, 1956

EAST COAST OF THE UNITED STATES

by

D. F. Bumpus Oceanographer

Woods Hole Oceanographic Institution

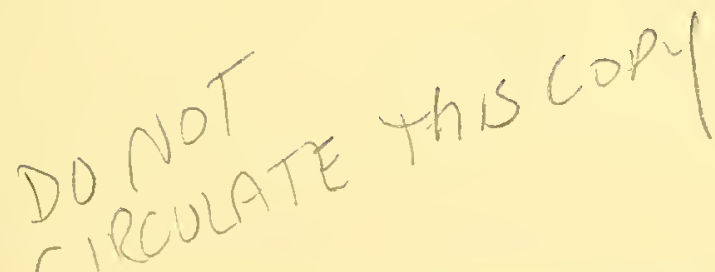

Special Scientific Report--Fisheries No. 233

ntribution No. 918 from the Woods Hole Oceanographic Institution (This work was supported by the U. S. Fish and Wildlife Service under Contract No. 14-19-008-2377)

Washington, D. C.

November 1957 
CONTENTS

Page

Abstract . . . . . . . . . . . . . . . . . . 1

Introduction . . . . . . . . . . . . . . . . . . . . 1

Commentary, figures and data... . . . . . . . . . 6

Grand Harbour, Grand Manan .. . . . . . . . . . 8

Portland Lightship . . . . . . . . . . . . . . . Il

Boston Lightship . . . . . . . . . . . . . . . 26

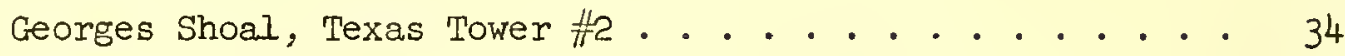

Nantucket Lightship . . . . . . . . . . . . . . 37

Woods Hole, Massachusetts . . . . . . . . . . . 51

Buzzards Lightship . . . . . . . . . . . . . 53

Kingston, Rhode Island ............ . 56

Ambrose Lightship . . . . . . . . . . . . . 58

Bamegat Lightship . . . . . . . . . . . . 66

Five Fathom Bank Lightship . . . . . . . . . . . 74

Winter Quarter Iightship . . . . . . . . . . 83

Chesapeake Lightship . . . . . . . . . . . . 92

Diamond Shoals Iightship . . . . . . . . . . . 101

Frying Pan Shoals Lightship . . . . . . . . . . . 116

Savannah Lightship . . . . . . . . . . . . 125

Literature Cited . . . . . . . . . . . . . . . 132 
LIST OF FIGURES

No.

Page

1. Locations along the Atlantic Coast reported herein . . . 4

2. Nomal cycle of temperature at surface and bottom along the string of observation posts for 1950 . . . . . . 7

3. Grand Harbour, Grand Manan . . . . . . . . . . . . 8

4. Portland Lightship . . . . . . . . . . . . . . 12

5. Boston Lightship . . . . . . . . . . . . . 26

6. Georges Shoal . . . . . . . . . . . . . 34

7. Nantucket Lightship . . . . . . . . . . . . . 38

8. Woods Hole . . . . . . . . . . . . . . . . . 58

9. Buzzards Lightship . . . . . . . . . . . . 53

10. Kingston . . . . . . . . . . . . 56

11. Ambrose Lightship . . . . . . . . . . . . . 59

12. Barnegat Iightship . . . . . . . . . . . . 66

13. Five Fathom Bank Lightship . . . . . . . . . . . 75

14. Vinter Quarter Lightship . . . . . . . . . . . 84

15. Chesapeake Lightship . . . . . . . . . . . . 93

16. Diamond Shoals Lightship . . . . . . . . . . . 102

17. Frying Pan Shoals Lightship . . . . . . . . . . . 117

18. Savannah Lightship . . . . . . . . . . . . 125 
No.

Page

1. Surface water temperatures-monthly and annual means for 1956 . . . . . . . . . . . . . . . . . 5

2. Grand Harbour . . . . . . . . . . . . . . . . 9

3. Fortland Lightship . . . . . . . . . . . . . . 13

4. Boston Lightship . . . . . . . . . . . . . . . 27

5. Georges Shoal . . . . . . . . . . . . . . 35

6. Nantucket Lightship . . . . . . . . . . . . . . 39

7. Voods Hole . . . . . . . . . . . . . . . . 52

6. Buzzards Lightship . . . . . . . . . . . . . . 54

9. Kingston . . . . . . . . . . . . . . . 57

10. Ambrose Lightship . . . . . . . . . . . . . 60

11. Bamegat Lightship . . . . . . . . . . . . 67

12. Five Fathom Bank Lightship . . . . . . . . . . . 76

13. Vinter Quarter Lightship . . . . . . . . . . . . 85

14. Chesapeake Lightship . . . . . . . . . . . . . . 94

15. Diamond Shoals Lightship . . . . . . . . . . . 103

16. Frying Pan Shoals Lightship . . . . . . . . . . 118

17. Savannah Lightship . . . . . . . . . . . . . . 126 
Oceanographic Observations, 1956

East Coast of the United States

\section{Abstract}

The daily water temperature and salinity observations fron sixteen locations along the Atlantic seaboard during 1956 are tabulated and discussed. Diagrams of the one-third monthly means are presented. This is the first time that it has been possible to present a synoptic description of the annual cycle of temperature and salinity for the coastal waters of the United States.

\section{Introduction}

Through the cooperation of the U. S. Coast Guard, the Woods Hole Oceanographic Institution established in late 1955 a series of oceanographic observation Posts at a number of the lightship stations along the east coast of the United States. Each lightship was furnished with a kit of oceanographic equipment and was requested to make the following observations:

Daily bathythermograph lowering.

Daily surface water temperature reading and surface water sample.

Daily record of air temperature, weather, wind and clouds.

Weekly bottom water sample.

These data have been forwarded on a monthly basis to Woods Hole where they have been processed. The bathythermograms have been read at several levels and tabulated in the accompanying tables. The surface water temperatures were used as a check on the bathythermograph. The water samples were titrated for salinity and tabulated. The records of air temperature, weather, wind and clouds were used in studying the other data, but are not presented here as they are the same as those published in the daily weather maps issued by the U. S. Weather Bureau. In addition to tabulating these data, mean temperatures for each level for 3 equal time periods per month have been determined and plotted as time profiles for the year for each station. The one-third monthly mean surface temperatures have also been plotted in comparison with the monthly mean surface temperature for the period of record of each station. The surface salinity one-third monthly means have been appended to the profiles, together with the weekly bottom salinity values.

The monthly mean surface water temperatures for the year 1956 for all stations are listed in Table 1 , for comparison with previous records (Bumpus, 1957). 
Certain independent observers have kindly forwarded to us daily water temperature readings at shore stations. These data are also tabulated herein and the one-third monthly means depicting the annual progression of surface water temperatures are presented.

The following stations are reported herein. (See Fig. 1)

Station

Period

Grand Harbour,

Grand Manan

Portland Iightship

Boston Lightship

12 Nov $1955-31$ Dec 1956

21 Apr 1956-31 Dec 1956

$3 \mathrm{Jan} 1956-12$ Dec 1956

Nantucket Lightship

Georges Shoal,

Texas Tower \#2

1 Aug 1955-31 Dec 1956

17 Dec $1955-28$ Dec 1956

3 Jan $1956-12$ Dec 1956

Woods Hole, Mass.

Buzzards Lightship

Kingston, R. I.

Ambrose Lightship

Barnegat Lightship

14 Dec $1955-16$ Dec 1956

2 Jan 1956-31 Dec 1956

1 Mar 1956-31 Dec 1956

6 Sent 1956-31 Dec 1956

$14_{4}$ Dec $1955-30$ Nov 1956

Five Fathom Bank

Lightship
15. Dec 1955-31 Dec 1956
Data

Tot

$\mathrm{T}_{01}, \mathrm{~T}_{30}, \mathrm{~T}_{50}, \mathrm{~T}_{100}$,

$T_{150}, S_{01}, S_{150}$

$T_{01}, T_{30}, T_{501}, T_{951}$,

$s_{01}, s_{951}$

$\mathrm{T}_{01}, \mathrm{~S}_{01}$

$\mathrm{T}_{0}, \mathrm{~T}_{30}$, $\mathrm{T}_{50}, \mathrm{~T}_{1001}$,

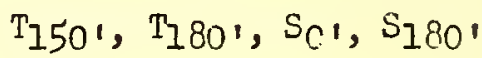

$T_{0}$

$\mathrm{T}_{\mathrm{O}}, \mathrm{S}_{\mathrm{O}}$

$T_{0}$

$$
\begin{aligned}
& \mathrm{T}_{01}, \mathrm{~T}_{30}, \mathrm{~T}_{501,} \mathrm{~T}_{951,} \\
& \mathrm{~S}_{01}, \mathrm{~S}_{95}, \\
& \mathrm{~T}_{0}, \mathrm{~T}_{30}, \mathrm{~T}_{501,} \mathrm{~T}_{70}, \\
& \mathrm{~S}_{01}, \mathrm{~S}_{70}
\end{aligned}
$$

$T_{0}, T_{30}, T_{50}, T_{85}$,

$\mathrm{S}_{0}, \mathrm{~S}_{851}$ 
Winter Quarter

Iight.ship

1 Dec 1955-31 Dec 1956

29 Nov 1955-29 Dec 1956

Diamond Shoals

Lightship

Frying Pan Shoals

Lightship

30 Nov 1955-31 Dec 1956

$18 . \operatorname{Tan} 1956-31$ Dec 1956
T01, T30', T50', T95',

$S_{01}, S_{95}$,

$T_{01}, T_{30}, T_{50}, T_{65}$,

$s_{0}, s_{65}$

$\mathrm{T}_{01}, \mathrm{~T}_{30}, \mathrm{~T}_{501}, \mathrm{~T}_{100}$,

$T_{150}, T_{180}, S_{0}, S_{180}$ '

$\mathrm{T}_{0}, \mathrm{~T}_{30}, \mathrm{~T}_{50}, \mathrm{~T}_{70}$,

$S_{0}, S_{70}$

$\mathrm{T}_{0}, \mathrm{~T}_{301}, \mathrm{~T}_{48}, \mathrm{~S}_{0}, \mathrm{~S}_{48}$, 


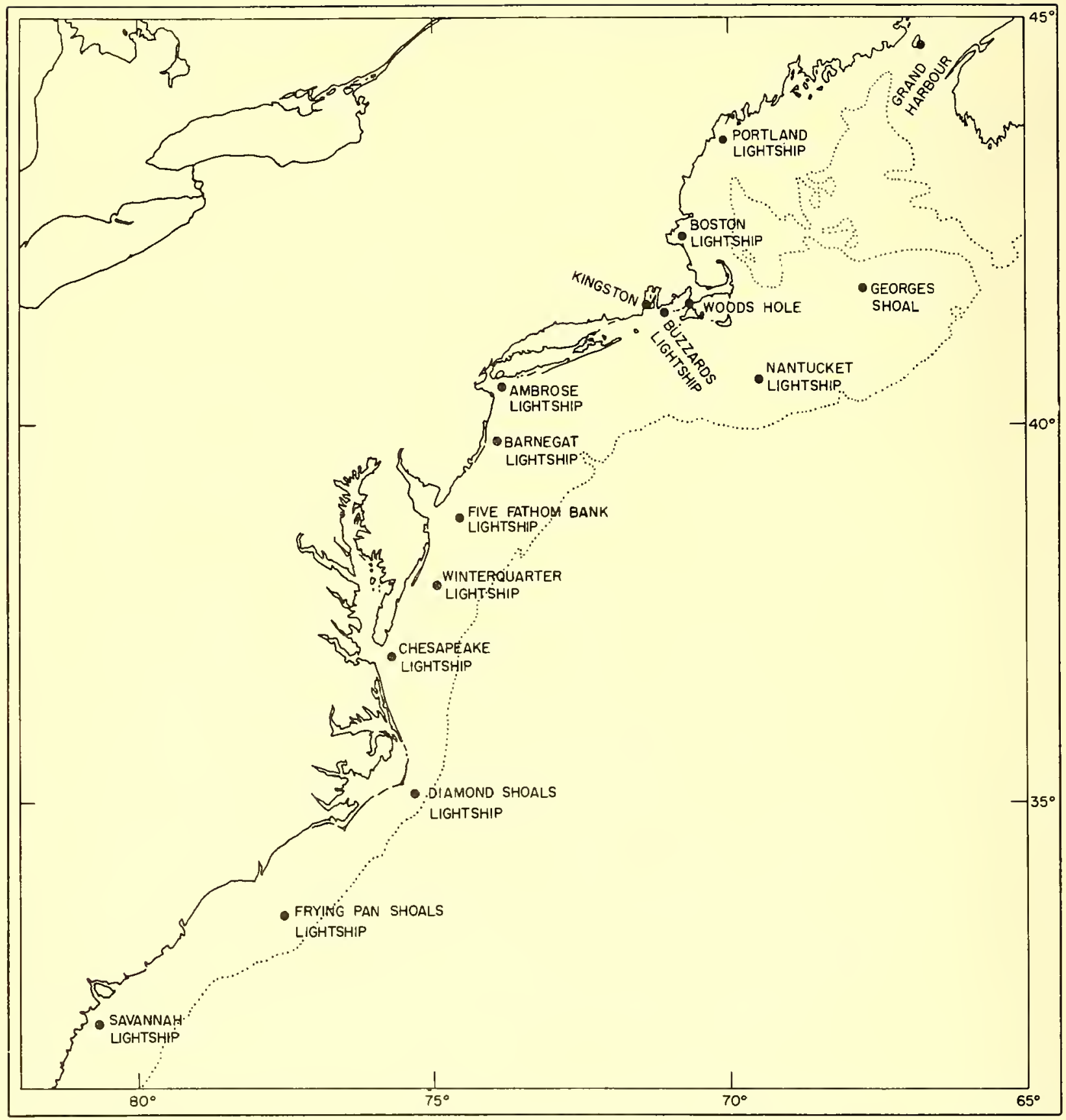

Figure 1. Locations along the Atlantic Coast reported herein. 


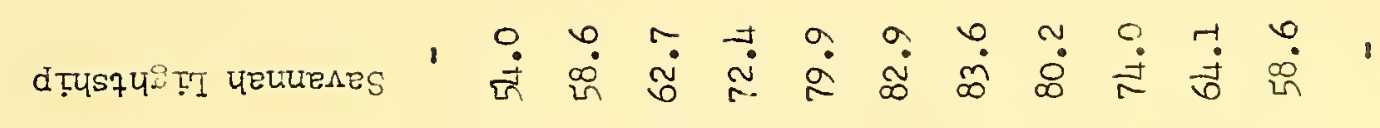

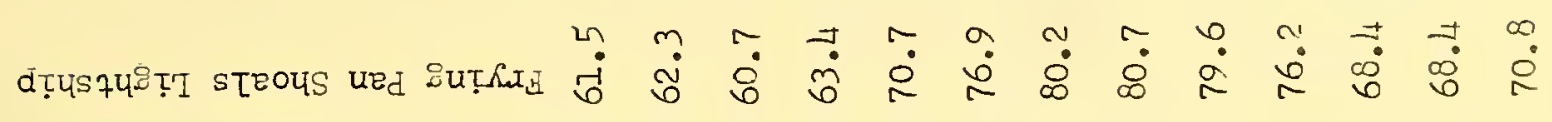

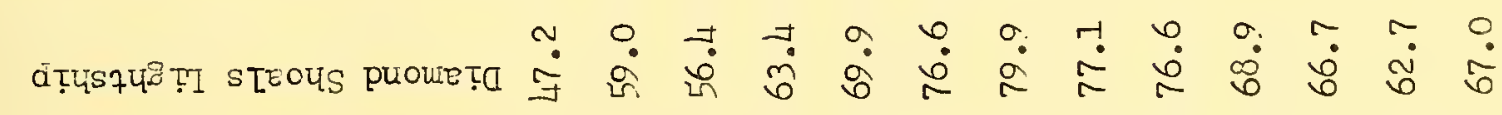

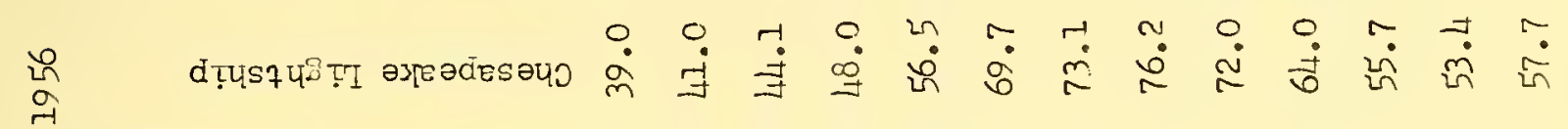

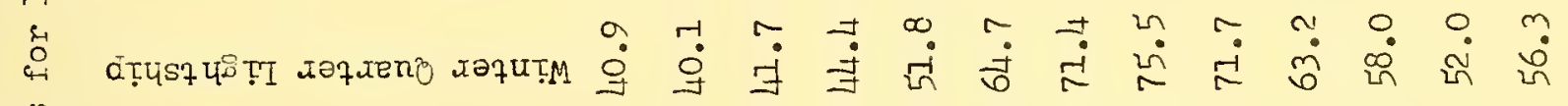

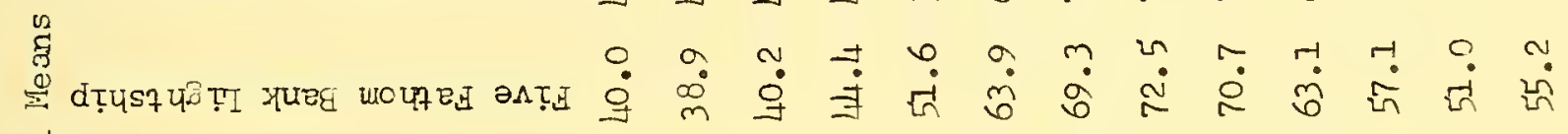
窎

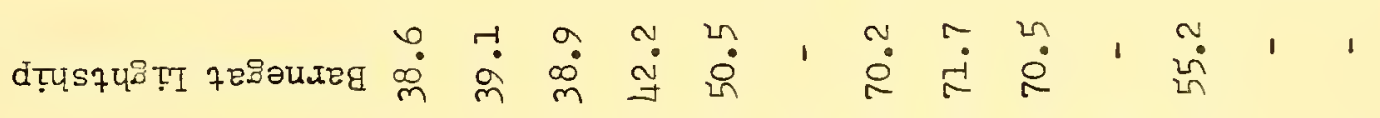
芯 ri
त्
0
0
0
0
E⿱

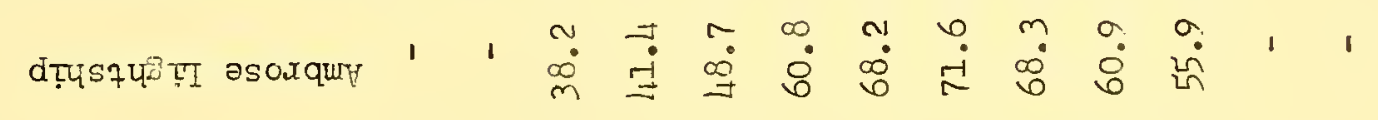

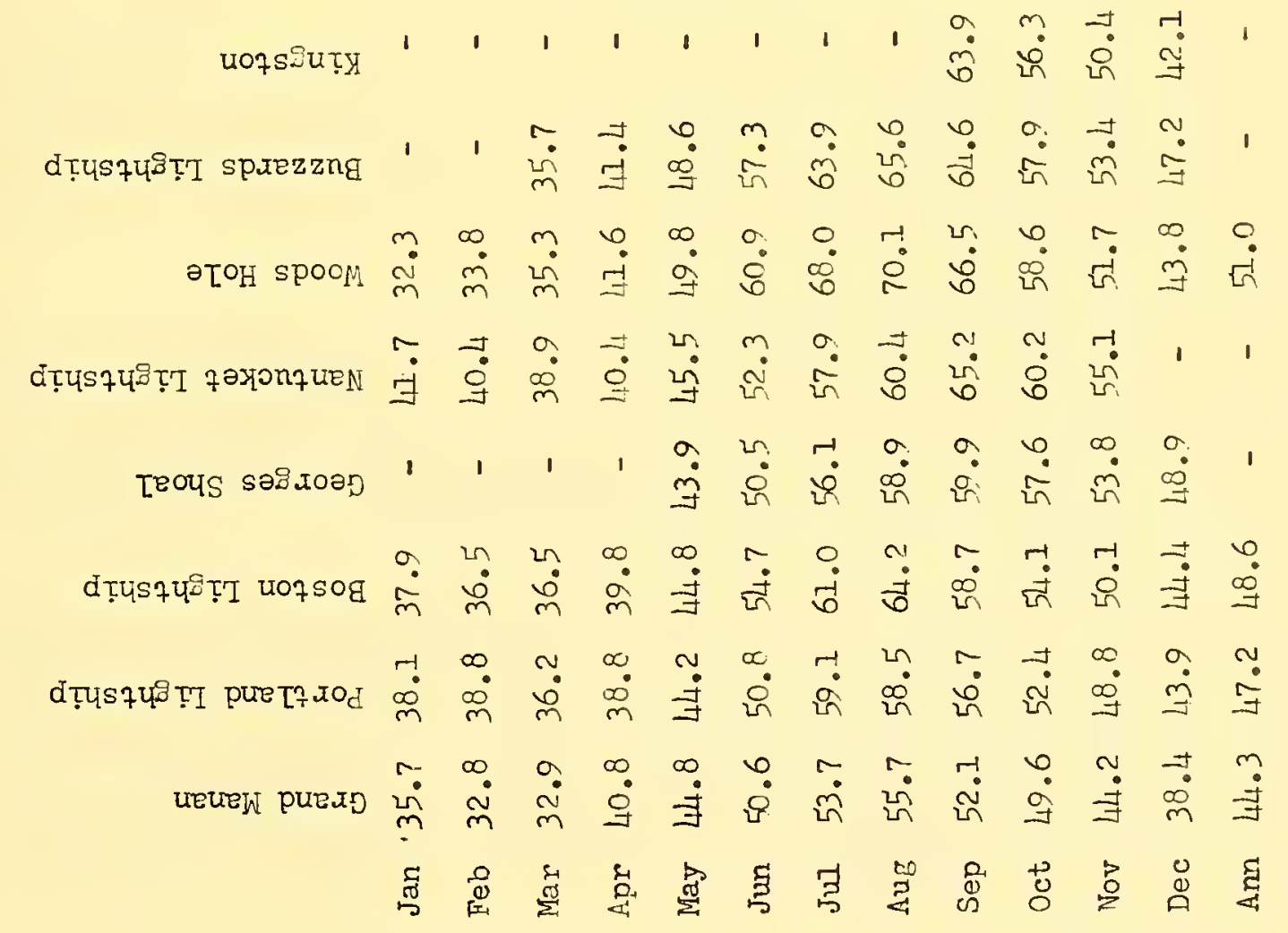


This is the first time that it has been possible to present a synoptic description of the temperature and salinity regime surface to bottom for the east coast of the United States. Rathbun (1887) and Parr (1933) have previously described and discussed the surface temperatures along this same coastline for earlier periods. Bumpus, 1957, has tabulated the monthly and annual means of all the available surface temperature data for the period of record.

We are particularly indebted to the U. S. Coast Guard personnel manning the lightships for their careful participation in this responsibility which is in addition to their regular chores; to Mr. Edward Green at Grand Harbour, Grand Manan; Mr. George Ferris at Woods Hole; Mr. Steacy Hicks at Kingston, R. I., and the l2th Weather squadron USAF at Georges Shoal and Otis AFB.

We are desirous of continuing the collection and publication of these data on an annual basis. We should be glad to include data of comparable nature from other locations were they available.

This work was supported by the U. S. Fish and Wildlife Service under Contract No. 14-19-008-2377 with the Woods Hole Oceanographic Institution.

\section{Commentary}

We have attempted below in the commentary for each station to point out the obvious features which characterize in general the hydrographic conditions during the year 1956. Obviously the biological investigator concerned with a particular group of plants or animals, or a more prescribed area, may wish to look to the data for more details than we have generalized here.

Parr (1933) went into some detail about the reliability of estimating the bottom temperature at lightship positions along the Atlantic Coast from the surface temperatures. He had no continuous records of bottom temperature at these positions. We now have, for the first time, a diagram of the surface and bottom temperature throughout the year (Figure 2) along the string of lightship positions from Portland to Savannah.

The major feature which Parr did not discuss is the displacement of the maximum bottom temperature to much later than the time of maximum surface temperature; from one to three months later at all locations from Diamond Shoals northward except Georges Shoals where the water is always isothermal. Because the thermocline is so weakly developed on the shelf south of Hatteras the time of maximum temperature is practically the same at the bottom as at the surface. 


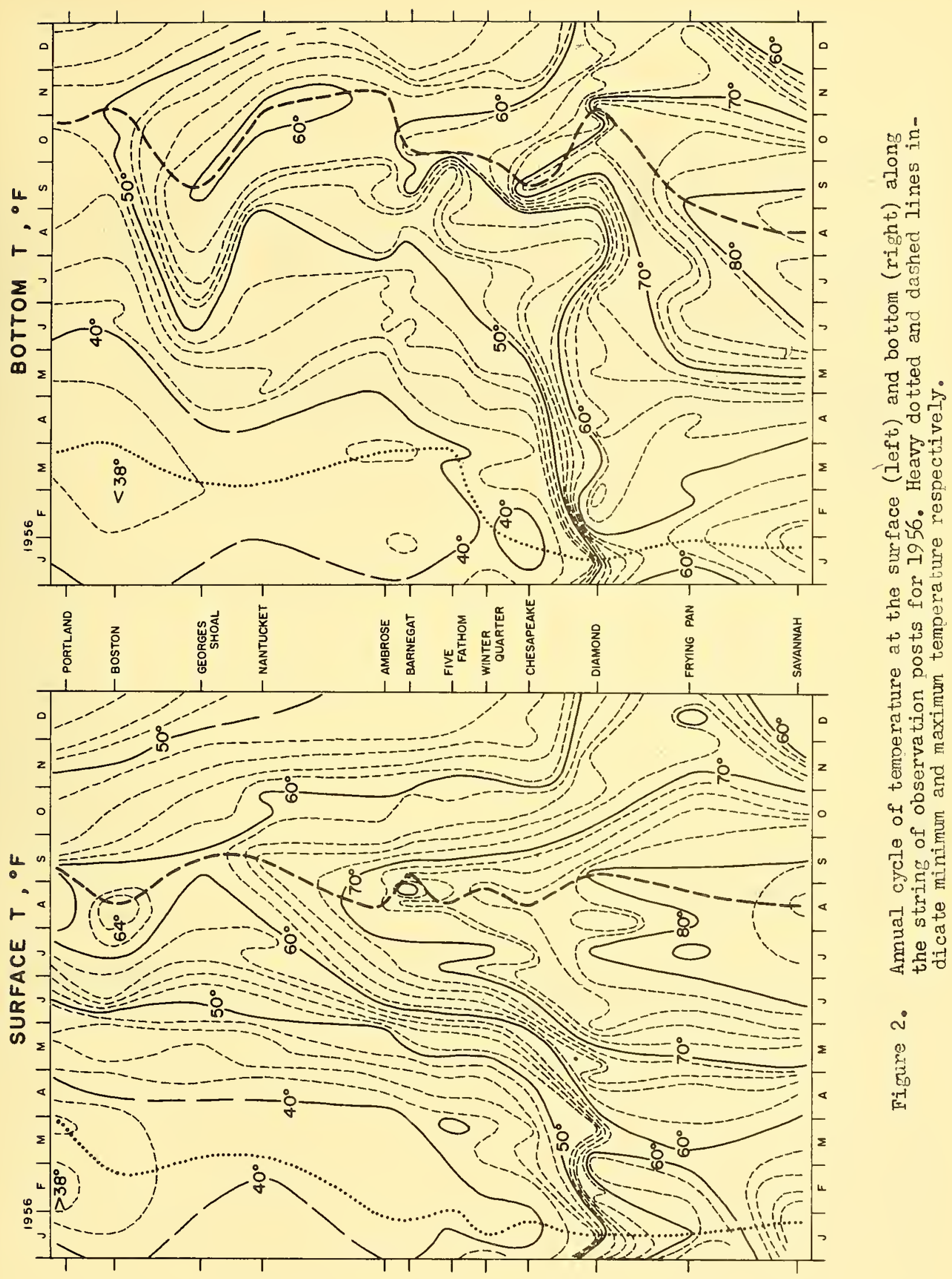


Grand Harbour, Grand Manan

The monthly means tabulated here sugrest that the temperatures in Grand Harbour are lower during the winter months and hi gher during the summer months than those recorded at Southwest Head, Grand Manan, almost eight miles away during the period 1929-1936 (Hachey, 1939). The annual mean temperatures are almost the same for both places.

Temperature fluctuations at Grand Harbour are subject to day to day variations of several desrees due to the broad expanse of shallow water where extrome heating or cooling may take place, the large tidal range and broad opening to the South and thoroughfare to the northeast permit substantial exchange with the open sea dependent on the wind and tide.

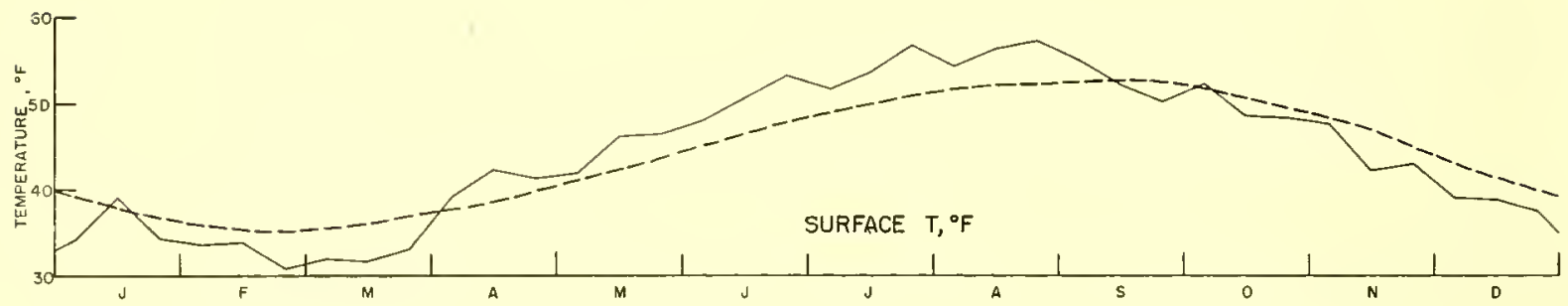

Fiture 3. Grand Harbor, Grand Manan, 1956 (thin line); Soithwest Head, Grand Manan, 1929-1936 (dashed line). 


\section{妾}

ơ

:

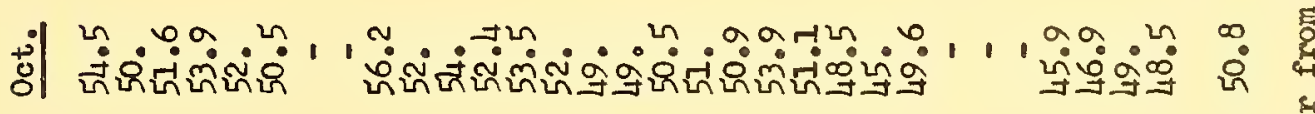

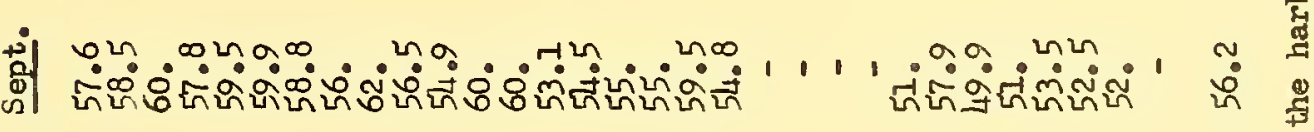

敞

ปี

ำ

㫄

害市

鿷

연

닌

这

ริ)

密

กง 
뎐|

$\stackrel{m}{\exists}$

岁

o|

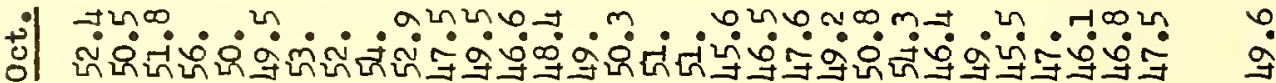

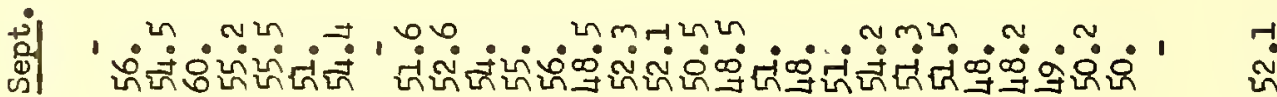

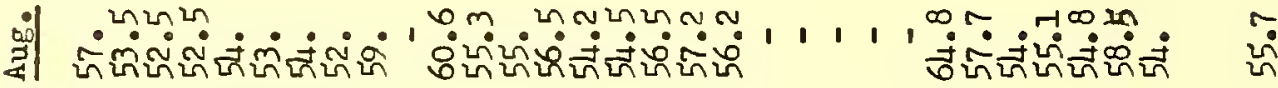

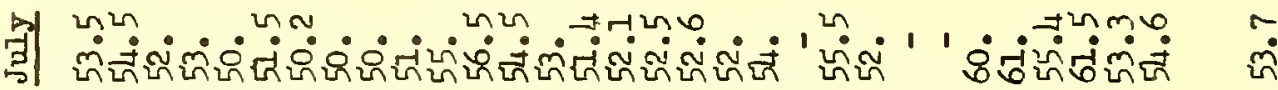

|)

囟

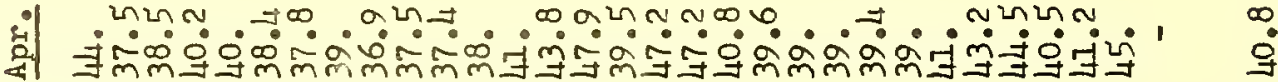

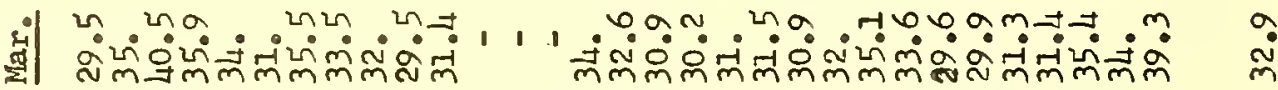

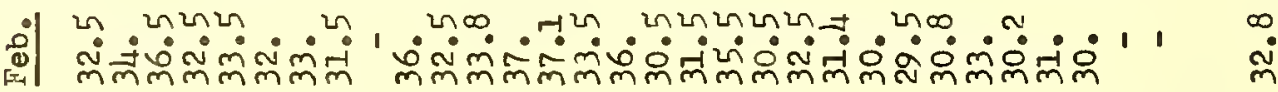

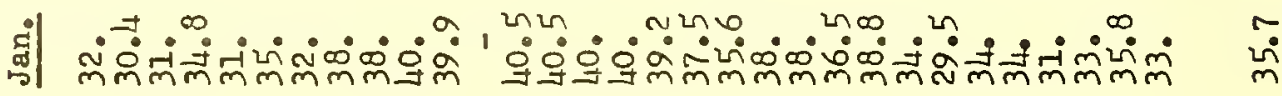

䗒 


\section{Portland Lightship}

Surface temperatures for 1956 were, on the whole, warmer than the means for the period 1925-1941, but cooler than the means for 1947-1956. (Bumpus, 1957).

Minimum temperatures occurred during the latter two-thirds of January with temperatures of slightly less than $37^{\circ} \mathrm{F}$ and during the last third of March at less than 360. By mid-April, a thermocline had begun to develop which reached a maximum in mid-August with surface temperatures in the high 50's and bottom temperatures in the low $40^{\prime} \mathrm{s}$. During the first third of September, the bottom temperatures suddenly increased 7 or $8^{\circ} \mathrm{F}$ and remained high, ca. $48^{\circ} \mathrm{F}$, until mid-November when temperatures at the surface hal dropped to such a level that complete turnover was takin place in the water column. Throughout the remainder of the year, temperatures between the surface and bottom were within a degree of each other, the surface being slightly cooler.

The year commenced with surface and bottom salinity at approximately 33\% 00 . The salinity gradually declined toward the end of March to ca.32\%/00. Beginning in April, the surface salinity lowered abruptly, reaching a minimum of $290 / 00$ during the first third of May. Surface salinity tended to remain less than 310/00 until August, followed by a gradual increase to $32.5 \% / 00$ at year end.

Dottom salinities tended to remain at about $32 \%$ between April and August but descended to less than $31 \% / 00$ during the first third of May and arain during the last half of July whereupon bottom salinities remained slightly higher than surface salinities until the last third of December. 

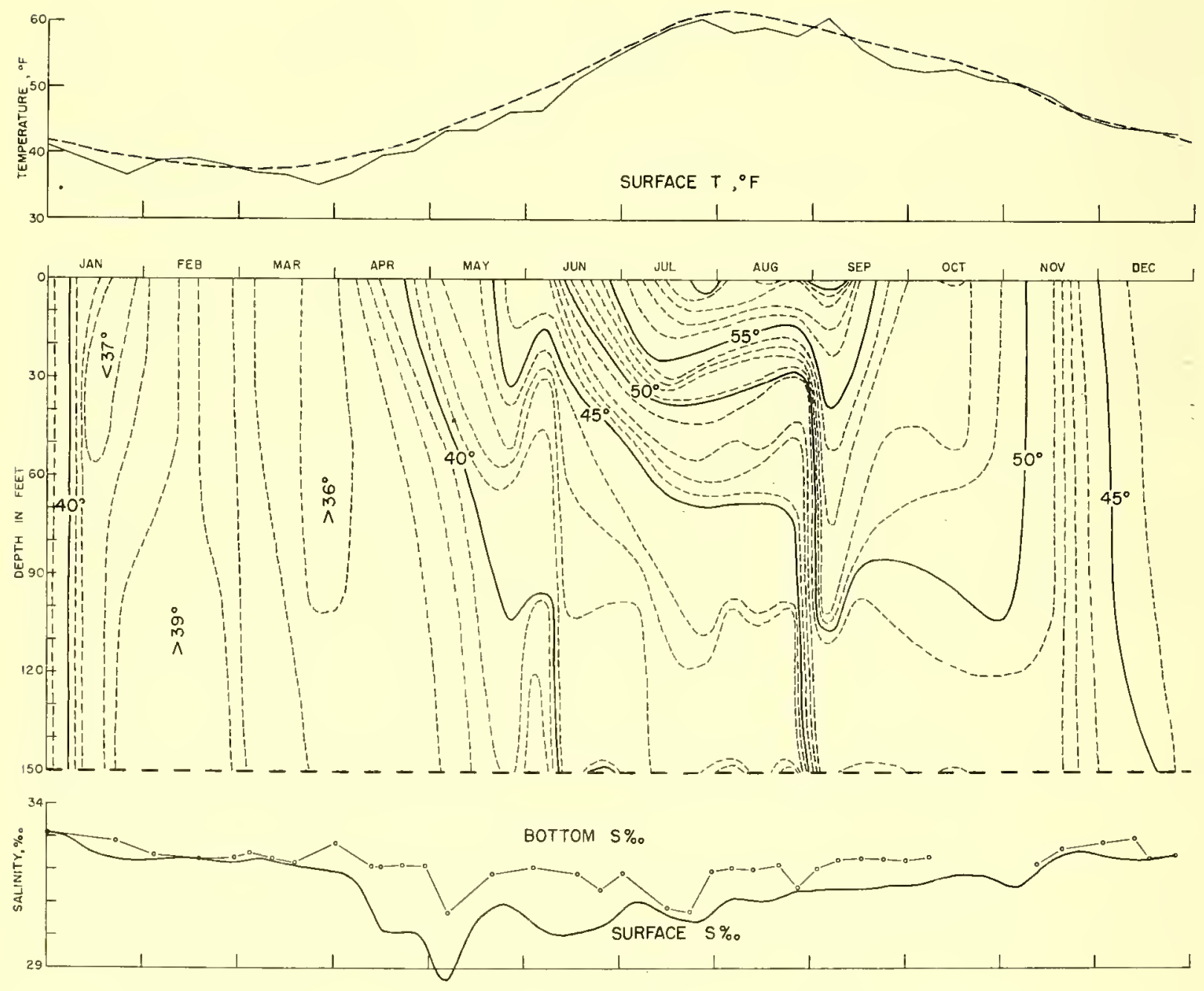

Figure 4. Portland Ijightship, 1956. (Dashed line in upper diagram, mean for period $1947-1,56)$ 
PORTIAND LIGHTSHIP $43^{\circ} 31.6 \mathrm{~N}^{\mathrm{N}} \cdot 70^{\circ} 05^{\circ} 5^{\mathrm{W}} \mathrm{W}$ Depth of water - 150 feet December 1955

\author{
Temperature ${ }^{\circ} \mathrm{F} \quad$ Salinity $\%$
}

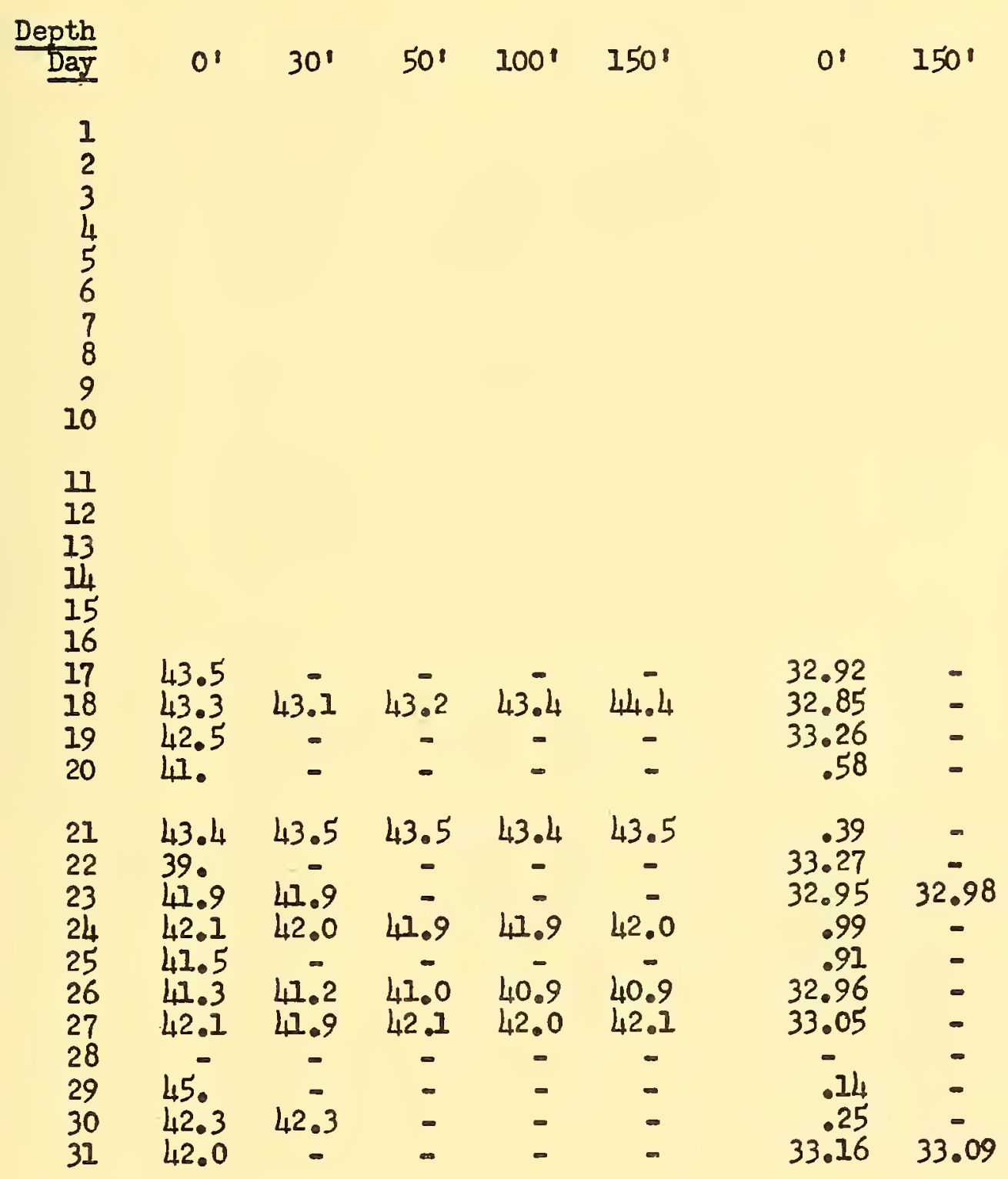

Mean 
PORTIAND LIGHTSHIP $43^{\circ} 31.6 \mathrm{~N}$. $70^{\circ} 05.5 \mathrm{w}$. Depth of water - 150 feet January 1956

$$
\text { Temperature oF }
$$

\section{$\frac{\text { Depth }}{\text { Day }}$}

$$
\begin{array}{r}
1 \\
2 \\
3 \\
4 \\
5 \\
6 \\
7 \\
8 \\
9 \\
10
\end{array}
$$

$\begin{array}{cccccc}11 & 40.7 & - & - & - & - \\ 12 & 38.1 & - & - & - & - \\ 13 & - & - & - & - & - \\ 14 & - & - & - & - & - \\ 15 & 38.1 & - & - & - & - \\ 16 & - & - & - & - & - \\ 17 & 42.5 & - & - & - & - \\ 18 & 36.7 & 37.0 & 37.2 & 37.2 & 37.1 \\ 19 & 36.5 & 36.5 & 36.4 & 37.5 & 38.0 \\ 20 & 36.2 & 36.7 & 36.9 & 37.3 & 38.0\end{array}$

$\begin{array}{llllll}21 & 36.4 & 36.8 & 37.7 & 38.3 & 39.0\end{array}$

$\begin{array}{llllll}22 & 37.2 & 36.9 & 37.2 & 38.6 & 39.4\end{array}$

$\begin{array}{llllll}23 & 35.0 & 38.5 & 38.9 & 39.6 & 39.4\end{array}$

$\begin{array}{llllll}24 & 36.8 & 36.8 & 36.9 & 39.9 & 40.0\end{array}$

$\begin{array}{llllll}25 & 35.9 & 36.1 & 39.1 & 39.4 & 39.9\end{array}$

$\begin{array}{llllll}26 & 37.9 & 38.1 & 38.1 & 39.4 & 40.4\end{array}$

2735.6 - $\quad$ - $\quad$ -

$\begin{array}{llll}28 & 35.5 & - & -\end{array}$

29

30

30

37.5

$-$

38.5 
PORTLAND LIGHTSHIP $43^{\circ} 31.6 \mathrm{~N} \cdot 70^{\circ} 05.5^{\prime} \mathrm{W}$. Depth of water -150 feet February 1956

Temperature OF

Depth $0^{\prime} \quad 30^{\prime} \quad 50^{\prime} 100^{\prime} \quad 150^{\prime}$

$\begin{array}{llllll}1 & 39.1 & 39.1 & 39.0 & 39.0 & 39.3\end{array}$

$\begin{array}{llllll}2 & 39.0 & 39.0 & 39.0 & 39.4 & 39.8\end{array}$

$\begin{array}{llllll}4 & 38.8 & 38.8 & 38.8 & 39.0 & 39.2 \\ 5 & 38.5 & 38.7 & 39.0 & 39.5 & -\end{array}$

6 - -2 - -

$\begin{array}{llllll}7 & - & - & - & - & -\end{array}$

$\begin{array}{llllll}9 & 39.0 & 38.8 & 38.5 & 38.8 & 39.2\end{array}$

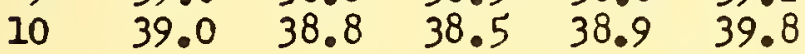

$\begin{array}{llllll}11 & 39.9 & 39.9 & 40.3 & 40.5 & 40.6\end{array}$

$\begin{array}{llllll}12 & - & - & - & - & -\end{array}$

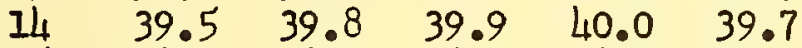

$\begin{array}{llllll}15 & 38.1 & 38.8 & 38.9 & 38.9 & 39.0\end{array}$

$\begin{array}{llllll}16 & 38.4 & 38.8 & 39.0 & 39.1 & 39.1\end{array}$

$\begin{array}{llllll}17 & 38.7 & 39.0 & 39.1 & 40.0 & -\end{array}$

$\begin{array}{llllll}18 & 39.1 & 39.1 & 39.2 & 39.5 & 39.8\end{array}$

$\begin{array}{llllll}19 & 39.6 & 39.6 & 39.5 & 39.5 & 39.5\end{array}$

$\begin{array}{llllll}20 & 38.5 & 38.8 & 39.0 & 39.0 & 39.2\end{array}$

$\begin{array}{llllll}21 & 38.5 & 38.7 & 39.0 & 39.8 & 40.0\end{array}$

$\begin{array}{llllll}22 & 38.4 & 38.5 & 38.6 & 39.1 & 39.5\end{array}$

$\begin{array}{llllll}23 & 38.2 & 38.2 & 38.2 & 39.3 & 39.7\end{array}$

$\begin{array}{llllll}24 & 37.9 & 37.9 & 37.9 & 38.9 & 39.9\end{array}$

25 - - - - -

26

27

28

29

30

31
Salinity $\%$

$0^{\prime} 150^{\prime}$

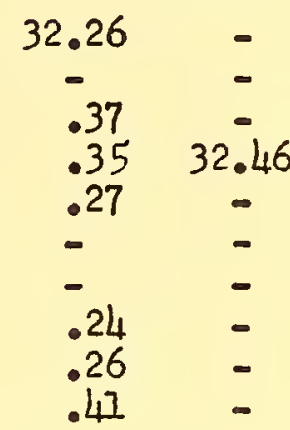

$.70 \quad-$

$\begin{array}{cc}. & = \\ .50 & = \\ .15 & = \\ .17 & = \\ .47 & = \\ .44 & = \\ .17 & 32.34 \\ .17 & = \\ .40 & =\end{array}$

74

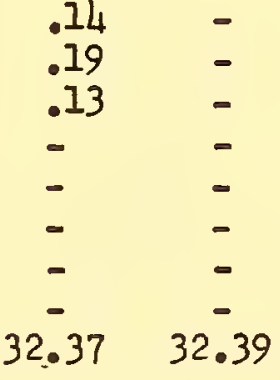

$\begin{array}{llllllll}\text { Mean } & 38.8 & 38.9 & 38.9 & 39.4 & 39.6 & 32.30 & 32.40\end{array}$ 
PORTLAND LIGHTSHIP $43^{\circ} 31.6 \mathrm{~N} \cdot 70^{\circ} 05.5 \mathrm{~W}$. Depth of water - 150 feet March 1956

$$
\text { Temperature }{ }^{O} \mathrm{~F}
$$

$\underline{\text { Depth }}$

Da

$\begin{array}{llllll}1 & 37.5 & 37.5 & 37.7 & 38.0 & 38.1 \\ 2 & 37.8 & 37.6 & 37.5 & 37.5 & 37.6\end{array}$

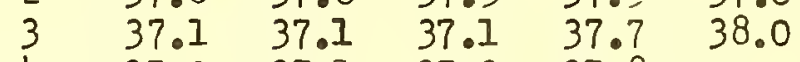

$\begin{array}{lllll}4 & 37.0 & 37.1 & 37.2 & 37.8\end{array}$

$\begin{array}{llllll}5 & 36.6 & 37.0 & 37.0 & 37.6 & 37.6\end{array}$

$\begin{array}{llllll}6 & 36.8 & 36.8 & 36.8 & 37.2 & 37.8\end{array}$

$\begin{array}{cccccc}7 & - & - & - & - & - \\ 8 & - & - & - & - & - \\ 9 & - & - & - & - & - \\ 10 & 36.4 & 36.2 & 36.3 & 37.7 & -\end{array}$

$\begin{array}{llllll}11 & 36.8 & 36.8 & 36.9 & 37.4 & 38.1\end{array}$

$\begin{array}{llllll}12 & 37.1 & 37.1 & 37.2 & 37.7 & 38.5\end{array}$

$\begin{array}{llllll}13 & 36.5 & 36.6 & 36.5 & 36.8 & 37.8\end{array}$

$\begin{array}{llllll}14 & 36.7 & 36.7 & 36.8 & 36.9 & 37.9\end{array}$

$\begin{array}{llllll}15 & 36.2 & 36.2 & 36.4 & 37.0 & 37.8\end{array}$

$\begin{array}{llllll}16 & 36.5 & 36.7 & 36.7 & 36.7 & 36.8\end{array}$

$\begin{array}{lllll}17 & - & - & - & -\end{array}$

19 - - - $=$

20

$\begin{array}{llllll}21 & 35.6 & 35.6 & 35.7 & 35.8 & 35.9\end{array}$

$\begin{array}{llllll}22 & 35.7 & 35.7 & 35.8 & 35.8 & 35.8\end{array}$

$\begin{array}{llllll}23 & 36.7 & 36.3 & 36.1 & 36.0 & 36.0\end{array}$

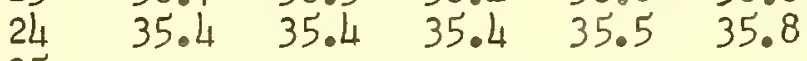

$25 \quad--3500$

$\begin{array}{llllll}26 & 34.7 & 34.2 & 34.9 & 35.2 & 35.9\end{array}$

$\begin{array}{llllll}27 & 34.8 & 34.5 & 34.9 & 35.8 & 36.1\end{array}$

$\begin{array}{llllll}28 & 34.5 & 34.4 & 34.4 & 35.2 & 36.3\end{array}$

$\begin{array}{llllll}29 & 35.0 & 34.8 & 34.8 & 35.2 & 36.4\end{array}$

$\begin{array}{llllll}30 & 34.9 & 34.9 & 34.9 & 35.9 & 36.3\end{array}$

$\begin{array}{llllll}31 & 35.0 & 35.0 & 35.5 & 36.3 & 36.4\end{array}$

$\begin{array}{llllll}\text { Mean } & 36.2 & 36.1 & 36.2 & 36.6 & 37.0\end{array}$
Salinity $\%$

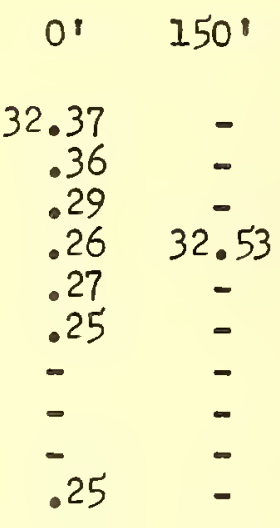

$.11 \quad 32.37$

$\begin{array}{ll}.17 & - \\ .17 & - \\ .18 & - \\ .05 & - \\ - & -\end{array}$

$.25 \quad 32.24$

-

$\begin{aligned} .22 & - \\ .14 & = \\ .80 & = \\ 32.15 & = \\ 31.74 & = \\ .73 & = \\ .86 & = \\ .84 & = \\ .85 & = \\ 31.79 & =\end{aligned}$

$32.13 \quad 32.38$ 
PORTLAND LIGHTSHIP $43^{\circ} 31.6^{\prime} \mathrm{N} \cdot 70^{\circ} 05.5 \mathrm{w}$. Depth of water - 150 feet April 1956

$$
\text { Temperature }{ }^{\circ} \mathrm{F}
$$

$\frac{\text { Depth }}{\text { Day }}$

Day

$\begin{array}{llllll}1 & 35.2 & 35.0 & 35.0 & 36.1 & 36.6\end{array}$

$235.9 \quad 35.3$

$3 \quad 36.0 \quad 35.8$

$4 \quad 36.4 \quad 36.0$

35.1

36.2

$35.8 \quad 35.6$

$36.8 \quad 36.4$

35.6

36.3

$7 \quad 37.1 \quad 37.0$

36.3

36.6

36.9

$36.3 \quad 36.8$

8

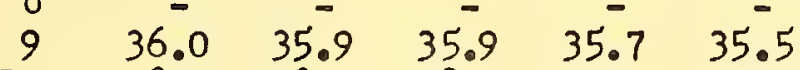

$\begin{array}{llllll}10 & 38.9 & 38.1 & 38.0 & 37.9 & 37.9\end{array}$

$\begin{array}{llllll}11 & 38.0 & 36.1 & 35.9 & 36.0 & 36.0\end{array}$

$\begin{array}{llllll}12 & 38.1 & 36.3 & 35.9 & 35.9 & 35.9\end{array}$

$\begin{array}{llllll}13 & 39.1 & 36.9 & 36.1 & 36.0 & 36.0\end{array}$

$\begin{array}{llllll}14 & 38.2 & 36.8 & 36.3 & 36.3 & 36.1\end{array}$

$\begin{array}{llllll}15 & 40.1 & 37.1 & 36.6 & 36.1 & 36.0\end{array}$

16 - $\quad$ - $\quad$ - $\quad$ -

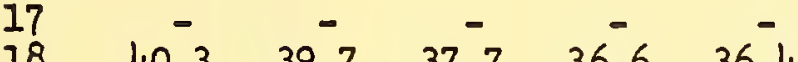

$\begin{array}{llllll}18 & 40.3 & 39.7 & 37.7 & 36.6 & 36.4\end{array}$

$\begin{array}{llllll}19 & 41.1 & 38.3 & 37.4 & 36.9 & 36.5\end{array}$

$\begin{array}{llllll}20 & 41.9 & 37.9 & 36.9 & 36.8 & 36.7\end{array}$

$\begin{array}{llllll}21 & 42.1 & 40.7 & 37.3 & 36.9 & 36.9\end{array}$

$\begin{array}{llllll}22 & 41.1 & 37.1 & 37.1 & 36.7 & 36.7\end{array}$

$\begin{array}{llllll}23 & 38.7 & 38.3 & 36.9 & 36.8 & 36.8\end{array}$

$\begin{array}{llllll}24 & 39.1 & 38.5 & 37.2 & 37.1 & 37.1\end{array}$

$\begin{array}{llllll}25 & 39.7 & 38.7 & 37.1 & 36.9 & 36.9\end{array}$

$\begin{array}{llllll}26 & 39.8 & 39.3 & 37.2 & 37.1 & 37.1\end{array}$

$\begin{array}{llllll}27 & 39.8 & 37.3 & 36.8 & 36.9 & 37.0\end{array}$

28 - $\quad-\quad$ - $\quad$ -

29 47. - - - -

$\begin{array}{llllll}30 & 40.1 & 39.3 & 38.7 & 37.2 & 37.1\end{array}$

31

Mean

$\begin{array}{lllll}38.8 & 37.3 & 36.6 & 36.5 & 36.6\end{array}$
Salinity $\%$

$0^{\prime} \quad 150^{\prime}$

$31.87 \quad 32.84$

32.23 -

31.81 -

$\begin{array}{rr}.70 & = \\ .58 & = \\ 31.68 & = \\ 32.48 & = \\ - & = \\ 31.79 & = \\ 31.10 & =\end{array}$

$29.50 \quad-$

$29.64 \quad 32.15$

30.66 =

$.65 \quad 32.10$

$\begin{array}{cc}- & - \\ .28 & - \\ 30.18 & - \\ 29.11 & -\end{array}$

$\begin{array}{lc}27.43 & - \\ 29.34 & 32.19\end{array}$

30.65 -

$.63=$

$.23=$

$.33=$

.53 -

$30.64 \quad 32.16$

31.23 -

$30.69 \quad 32.29$ 
PORTLAND LIGHTSHIP $43^{\circ} 31.6^{\prime} \mathrm{N} .70^{\circ} 05.5^{\prime} \mathrm{W}$. Depth of water - 150 feet

\section{May 1956}

Temperature ${ }^{\circ} \mathrm{F}$

Depth

Day

147.5

42.2

43.3

45.6

43.3

6 4 43.8

843.2

944.2

10

$\begin{array}{llllll}11 & 41.2 & 40.7 & 40.4 & 39.1 & 38.1\end{array}$

12

13

14

15

16

17

18

19

20

$\begin{array}{lllllll}21 & 46.4 & 45.1 & 42.6 & 40.0 & 39.1\end{array}$

$\begin{array}{llllll}22 & 46.5 & 45.0 & 43.0 & 40.2 & 39.0\end{array}$

23

24

25

26

27

29

30

31
$43.2 \quad 41.7 \quad 40.0 \quad 38.1 \quad 38.0$

$\begin{array}{lllll}43.6 & 43.5 & 47.2 & 38.8 & -\end{array}$

43.5

44.

47.6

42.3

4.1

44.

44.0

45.0

43.8

43.9

43.9

44.0

39.8

40.3

38.5

39.8

39.7

42.6

43.6

43.1

40.0

38.938 .3

40.2

$=$

-

45.4

46.0

45.8

47.7

$-$

45.1

45.2

45.

46.2
$-$

40.7

44.7

45.2

44.7

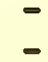

Salinity $\%$

$0^{\prime} \quad 150^{\prime}$

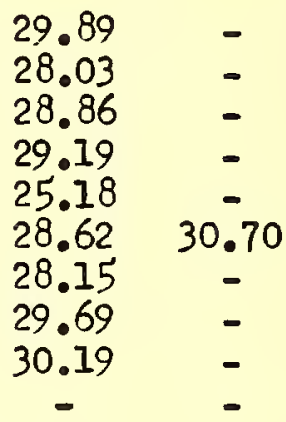

$31.17=$

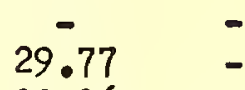

30.36 -

30.54 -

29.96 -

$30.49=$
$.75=$

$.60 \quad-$

.60 .91
$-\quad 31.9$

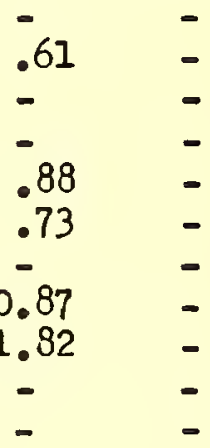

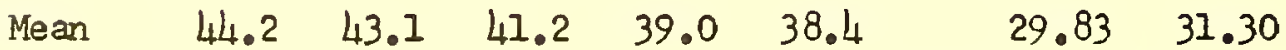


PORTLAID LIGHTSHP $43^{\circ} 31.6^{\prime} \mathrm{N} \cdot 70^{\circ} 05.5^{\prime} \mathrm{W}$. Depth of water -150 feet June 1956

$$
\text { Temperature }{ }^{\circ} \mathrm{F}
$$

Salinity $\%$

Depth

$\begin{array}{llllll}1 & 45.6 & 42.5 & 40.4 & 37.1 & 37.0\end{array}$

$245.0-10$.

$345.0-$

$\begin{array}{llllll}4 & 46.6 & 42.0 & 39.7 & 37.2 & -\end{array}$

$\begin{array}{llllll}6 & 46.8 & 47.3 & 39.7 & 38.7 & 37.5\end{array}$

$\begin{array}{llllll}7 & 48.0 & 42.1 & 40.7 & 39.9 & 38.8\end{array}$

$\begin{array}{llllll}8 & 47.5 & 42.7 & 40.9 & 39.3 & 38.9\end{array}$

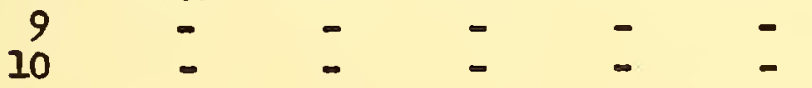

$\begin{array}{llllll}17 & 44.1 & 42.5 & 42.5 & 47.4\end{array}$

$\begin{array}{lllll}12 & 46.9 & 41.9 & 42.2 & 47.5\end{array}$

$\begin{array}{lllll}13 & 52.2 & 43.4 & 42.0 & 41.5\end{array}$

$\begin{array}{llllll}14 & 46.5 & 44.2 & 43.0 & 42.1 & - \\ 15 & 56.8 & 44.2 & 42.9 & 42.0 & 40.1\end{array}$

$\begin{array}{lllll}16 & 50.2 & 47.6 & 45.5 & 44.5\end{array}$

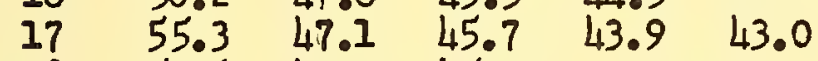

$\begin{array}{lllll}18 & 5.6 \quad 47.0 \quad 45.1 \quad\end{array}$

$\begin{array}{llllll}19 & 54.9 & - & - & - & -\end{array}$

$21 \quad 51.0 \quad 50.0 \quad 45.6 \quad-\quad$ -

$\begin{array}{llllll}22 & 53 . & 50 . & - & -\end{array}$

$\begin{array}{llllll}24 & 53.8 & 45.5 & 44.7 & 47.6 & 39.9\end{array}$

$\begin{array}{llllll}25 & 54.0 & 49.5 & 44.2 & 42.7 & -\end{array}$

$\begin{array}{llllll}26 & 55.5 & 50.9 & 44.0 & 47.7 & -\end{array}$

$\begin{array}{llllll}27 & 55.4 & 46.7 & 44.1 & 47.9 & 40.1\end{array}$

$\begin{array}{llllll}28 & 54.7 & 50.3 & 45.4 & 47.8 & -\end{array}$

$\begin{array}{llllll}29 & 54.5 & - & - & - & -\end{array}$

Mean

$\begin{array}{lllll}50.8 & 45.6 & 43.0 & 47.1 & 39.4\end{array}$

$30.19 \quad 31.82$ 
PORTLAND LIGHTSHIP $43^{\circ} 31.6 \mathrm{~N} \cdot 70^{\circ} 05.5^{\mathrm{N}} \mathrm{N}$. Depth of water - 150 feet July 1956

$$
\text { Temperature oF }
$$

Salinity $\%$

Depth

Day

$0^{\prime} \quad 30^{\prime} 50^{\prime}$ ìn' $150^{\prime}$

$0^{\prime} 150^{\prime}$

$\begin{array}{llllll}1 & 58.2 & 49.7 & 44.7 & 42.4 & 41.7\end{array}$

$\begin{array}{llllll}2 & 53.9 & 52.3 & 45.3 & 42.7 & 41.5\end{array}$

$\begin{array}{llllll}3 & 56.0 & 45.6 & 43.8 & 42.1 & -\end{array}$

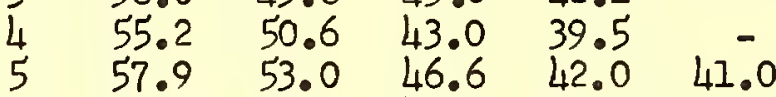

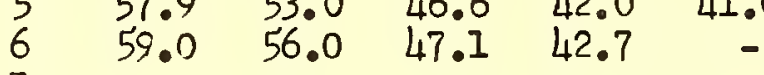

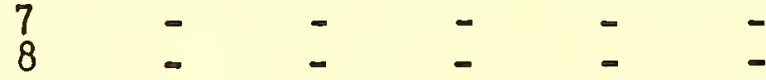

$\begin{array}{lllll}9 & - & - & - & -\end{array}-$

$\begin{array}{llllll}11 & 57.1 & 51.2 & 48.7 & 44.0 & 42.4\end{array}$

$\begin{array}{llllll}12 & 58.0 & 52.8 & 47.9 & 44.6 & 42.5\end{array}$

13 - $\quad$ - $\quad$ - $\quad$ -

$\begin{array}{llllll}14 & - & - & - & - & -\end{array}$

$\begin{array}{llllll}16 & 59.7 & 57.4 & 47.0 & 43.8 & -\end{array}$

$\begin{array}{llllll}17 & 57.8 & 56.9 & 46.9 & 43.7 & =\end{array}$

$\begin{array}{lllll}18 & 60.5 & 56.8 & 51.0 & 43.8\end{array}$

$\begin{array}{lllll}19 & 60.0 & 5.7 & 48.0 & 47.7\end{array}$

$\begin{array}{lllll}20 & 61.8 & 53.6 & 48.3 & 44.9\end{array}$

$\begin{array}{llllll}21 & 64.6 & 51.2 & 47.0 & 44.8 & 42.0\end{array}$

22 - - - -

$\begin{array}{lllll}23 & 58.0 & 48.1 & 46.9 & 44.3\end{array}$

$\begin{array}{lllll}24 & 56.0 & 50.5 & 47.6 & 45.0\end{array}$

25 60. 51.249 .1 - -

$2659 . \quad-\quad-\quad-$

$\begin{array}{llllll}27 & 60 . & - & - & - & -\end{array}$

29 62. - $\quad-\quad-\quad-$

$\begin{array}{lllll}30 & 61 . & - & - & -\end{array}$

$\begin{array}{llllllll}\text { Mean } & 59.1 & 52.8 & 47.3 & 43.4 & (42.0) & 30.65 & 31.38\end{array}$ 
PORTLAID LIGHTSHIP $43^{\circ} 31.61 \mathrm{~N} \cdot 70^{\circ} 05.5 \mathrm{~W}$. Depth of water - 150 feet

\section{August 1956}

$$
\text { Temperature oF }
$$

$\frac{\text { Depth }}{\text { Day }}$

$0^{\prime} \quad 30^{\prime} \quad 50^{\prime} 100^{\prime} 150^{\prime}$

$\begin{array}{lllll}1 & 61.8 & 55.3 & 52.6 & 45.2\end{array}$

$\begin{array}{lllll}2 & 61.0 & 54.5 & 47.8 & 42.0\end{array}$

$\begin{array}{lllll}3 & 57.0 & 49.3 & 45.5 & 47.3\end{array}$

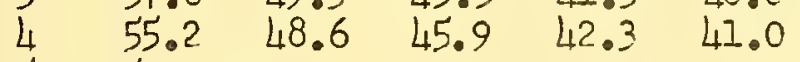

5 57. - - - -

659 - 5 - -2

$\begin{array}{lllll}7 & 57.8 & 49.0 & 46.7 & 43.0\end{array}$

$\begin{array}{llllll}8 & 50.9 & 50.2 & 47.0 & 42.9 & -\end{array}$

$\begin{array}{lllll}10 & 60.0 & 51.7 & 49.5 & 44.3\end{array}$

$\begin{array}{llllll}11 & 56.5 & 48.9 & 47.0 & 43.8 & \text { - }\end{array}$

$\begin{array}{llll}12 & 59.0 \quad 50.2 \quad 47.9 \quad 44.0\end{array}$

13 - $\quad$ - - - -

14

$\begin{array}{llllll}15 & - & - & - & - & -\end{array}$

$\begin{array}{lllll}17 & 61.2 & 55.0 & 51.2 & 46.8\end{array}$

$\begin{array}{lllll}18 & 62.0 & 52.4 & 49.3 & 45.0\end{array}$

$\begin{array}{lllll}19 & - & - & - & -\end{array}$

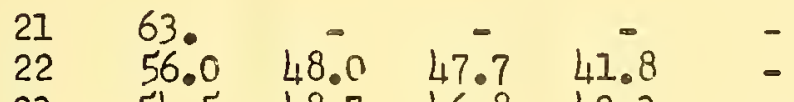

$\begin{array}{lllll}23 & 54.5 & 48.7 & 46.8 & 42.3\end{array}$

$\begin{array}{llllll}24 & - & - & - & - & -\end{array}$

26 60. - - - -

2757.0 - -

28 56.5 - - - -

$2960 . \quad-\quad-$

30 60: $\quad-\quad-\quad-5$

Mean

$$
\begin{array}{lllll}
58.5 & 50.8 & 47.9 & 43.4 & 40.7
\end{array}
$$

Salinity $\%$

$0^{\prime} \quad 150^{\prime}$

$\begin{array}{rc}30.72 & - \\ 30.82 & - \\ 31.07 & - \\ .15 & - \\ .78 & 32.06 \\ 31.23 & - \\ 30.25 & - \\ - & - \\ 31.43 & - \\ .82 & -\end{array}$

$31.10 \quad 32.05$

$\begin{array}{cc}- & = \\ - & = \\ 30.06 & = \\ 30.85 & = \\ 31.49 & 32.20\end{array}$

$31.44 \div \quad 32.20$

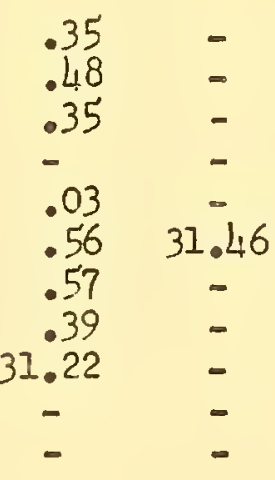

$31.20 \quad 31.94$ 
PORTIAND LIGHTSHIP $43^{\circ} 31.6 \mathrm{~N} .70^{\circ} 05.5 \mathrm{~m}$. Depth of water - 150 feet

\section{September 1956}

\begin{tabular}{|c|c|c|c|c|c|c|c|}
\hline \multirow[b]{2}{*}{$\frac{\text { Depth }}{\underline{\text { Day }}}$} & \multicolumn{5}{|c|}{ Tenperature oF } & \multicolumn{2}{|c|}{ Salinity $\% / 00$} \\
\hline & $0^{\prime}$ & $30^{\prime}$ & $50^{\prime}$ & $100^{\prime}$ & $150^{\prime}$ & $0^{\prime}$ & $150^{\prime}$ \\
\hline $\begin{array}{r}1 \\
2 \\
3 \\
4 \\
5 \\
6 \\
7 \\
8 \\
9 \\
10\end{array}$ & $\begin{array}{l}61.7 \\
62.8 \\
62.2 \\
64.0 \\
60.9 \\
62.0 \\
62.0 \\
59.8 \\
57.5 \\
56.5\end{array}$ & $\begin{array}{l}60.7 \\
60.5 \\
60.1 \\
61.0 \\
57.2 \\
58.0 \\
58.8 \\
58.4 \\
57.2 \\
55.1\end{array}$ & $\begin{array}{l}59.7 \\
57.3 \\
58.3 \\
58.9 \\
54.5 \\
56.8 \\
56.1 \\
54.5 \\
55.0 \\
53.8\end{array}$ & $\begin{array}{l}50.0 \\
49.1 \\
49.8 \\
51.7 \\
59.7 \\
53.3 \\
51.2 \\
50.9 \\
50.9 \\
49.1\end{array}$ & $\begin{array}{l}48.0 \\
48.7 \\
47.8 \\
49.0 \\
48.5 \\
50.0 \\
48.9 \\
48.6 \\
48.0 \\
47.0\end{array}$ & $\begin{array}{r}31.64 \\
.65 \\
.55 \\
.18 \\
.67 \\
.19 \\
.17 \\
.67 \\
.37 \\
.46\end{array}$ & $\begin{array}{c}32.08 \\
- \\
- \\
- \\
- \\
32.33 \\
-\end{array}$ \\
\hline $\begin{array}{l}11 \\
12 \\
13 \\
14 \\
15 \\
16 \\
17 \\
18 \\
19 \\
20\end{array}$ & $\begin{array}{l}55.4 \\
59.7 \\
57.0 \\
56.7 \\
55.8 \\
53.1 \\
56.5 \\
56.0 \\
55.8 \\
54.0\end{array}$ & $\begin{array}{l}53.2 \\
55.3 \\
54.9 \\
56.3 \\
55.5 \\
52.8 \\
5 \\
56.0 \\
54.8 \\
53.9\end{array}$ & $\begin{array}{l}51.6 \\
53.5 \\
53.2 \\
50.8 \\
53.2 \\
50.3 \\
- \\
55.7 \\
52.6 \\
52.7\end{array}$ & $\begin{array}{l}46.1 \\
51.1 \\
50.6 \\
47.1 \\
47.9 \\
46.4 \\
50.2 \\
49.5 \\
49.5\end{array}$ & $\begin{array}{c}48.6 \\
- \\
46.2 \\
46.9 \\
44.3 \\
- \\
49.0 \\
48.7 \\
48.5\end{array}$ & $\begin{array}{l}.16 \\
.48 \\
.31 \\
.45 \\
.59 \\
.58 \\
.59 \\
.63 \\
.56 \\
.39\end{array}$ & $\begin{array}{c}- \\
- \\
- \\
- \\
32.35 \\
= \\
- \\
-\end{array}$ \\
\hline $\begin{array}{l}21 \\
22 \\
23 \\
24 \\
25 \\
26 \\
27 \\
28 \\
29 \\
30\end{array}$ & $\begin{array}{l}52.9 \\
53.1 \\
53.3 \\
54.7 \\
55.0 \\
54.4 \\
51.8 \\
51.9 \\
52.2 \\
53.5\end{array}$ & $\begin{array}{l}52.9 \\
52.3 \\
52.9 \\
54.6 \\
54.2 \\
54.0 \\
51.8 \\
51.6 \\
51.8 \\
52.2\end{array}$ & $\begin{array}{l}51.7 \\
49.0 \\
51.0 \\
53.3 \\
53.8 \\
53.6 \\
51.7 \\
51.5 \\
51.7 \\
52.0\end{array}$ & $\begin{array}{l}48.1 \\
47.9 \\
48.6 \\
50.4 \\
1.9 .7 \\
50.4 \\
49.7 \\
49.0 \\
49.2 \\
50.2\end{array}$ & $\begin{array}{l}47.3 \\
46.9 \\
47.2 \\
49.2 \\
47.9 \\
48.9 \\
47.0 \\
47.5 \\
47.5 \\
49.1\end{array}$ & $\begin{array}{r}.61 \\
.74 \\
.66 \\
.68 \\
.80 \\
.82 \\
.58 \\
.42 \\
.41 \\
31.21\end{array}$ & $\begin{array}{c}- \\
- \\
32.35 \\
- \\
- \\
= \\
- \\
- \\
- \\
-\end{array}$ \\
\hline
\end{tabular}

$\begin{array}{llllllll}\text { Mean } & 56.7 & 55.4 & 53.7 & 49.6 & 48.0 & 31.51 & 32.29\end{array}$ 
PORTLAND LIGHTSHIP $43^{\circ} 31.6^{\prime} \mathrm{N} .70^{\circ} 05.5 \mathrm{~W}$. Depth of water - 150 feet

\section{October 1956}

$$
\text { Temperature }{ }^{\circ} \mathrm{F}
$$

Depth

$\begin{array}{rrrrrc}\text { Day } & 0^{\prime} & 30^{\prime} & 50^{\prime} & 100^{\prime} & 150^{\prime} \\ 1 & 54.9 & 53.9 & 53.2 & 51.3 & 50.0 \\ 2 & 54.9 & 52.7 & 52.2 & 51.5 & - \\ 3 & 54.2 & 54.1 & 53.8 & 51.3 & 48.9 \\ 4 & 52.8 & 52.5 & 50.9 & 49.1 & 47.4 \\ 5 & 53.2 & 53.0 & 52.9 & 51.1 & 47.9 \\ 6 & 53.0 & 52.2 & 52.5 & 50.5 & - \\ 7 & 53.6 & 53.1 & 52.8 & 50.4 & - \\ 8 & 47.9 & 47.8 & 47.2 & 44.1 & - \\ 9 & 53.9 & 53.8 & 53.1 & 49.3 & 47.9 \\ 10 & 48.1 & 47.6 & 47.1 & 43.8 & -\end{array}$

11

12

13

14

15

16

1753

18

19

$\begin{array}{llllll}21 & 52.0 & 51.8 & 5.8 & 48.9 & 47.4\end{array}$ $\begin{array}{llllll}22 & 52.3 & 52.0 & 51.4 & 48.8 & 47.1\end{array}$

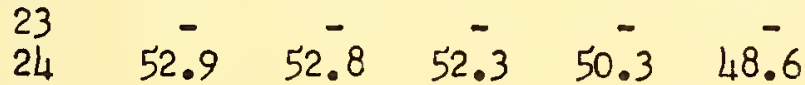
$25-0-30$.

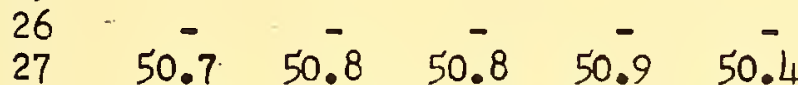

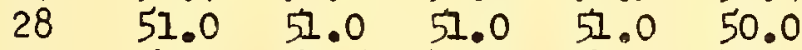

$\begin{array}{llllll}29 & 50.7 & 50.5 & 49.9 & 50.2 & 49.6\end{array}$

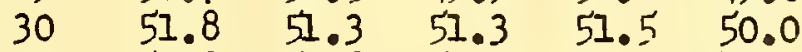

$\begin{array}{llllll}31 & 50.8 & 50.8 & 50.1 & 50.0 & 47.7\end{array}$

$\begin{array}{llllll}\text { Mean } & 52.4 & 52.0 & 51.6 & 49.6 & 48.4\end{array}$
Salini ty $\%$

$0^{\prime} 150^{\prime}$

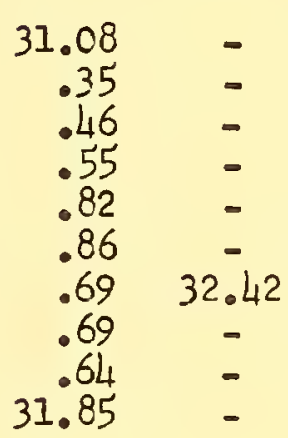

$\begin{array}{cc}- & = \\ - & = \\ 31.94 & - \\ .78 & = \\ .66 & = \\ .95 & = \\ 31.89 & - \\ -84 & = \\ 32.00 & =\end{array}$

$\begin{array}{cc}31.98 & = \\ .88 & = \\ 0 & = \\ .95 & = \\ - & = \\ .79 & = \\ 31.92 & = \\ 32.46 & = \\ 31.92 & = \\ - & =\end{array}$

$31.74 \quad(32.42)$ 
PORTLAND LIGHTSHIP $43031.6 \mathrm{~N} \cdot 70005.5 \mathrm{~W}$. Depth of water - 150 feet

\section{November 1956}

$$
\text { Temperature of }
$$

Deoth $\begin{array}{llllll}1 & 51.1 & 50.8 & 50.7 & 50.1 & 48.5\end{array}$

$$
5
$$

2

25

$0^{\prime} \quad 30^{\prime} \quad 50^{\prime} 100^{\prime} 150^{\prime}$ - - - - $\begin{array}{ccccc}50.7 & 50 . \overline{4} & 50.3 & 50.2 & 48.7\end{array}$ $\begin{array}{lllll}50.6 & 50.2 & 50.0 & 49.7 & 48.2\end{array}$ $\begin{array}{lllll}50.5 & 50.2 & 50.0 & 49.7 & 47.7\end{array}$ $\begin{array}{lllll}50.4 & 50.2 & 50.0 & 49.4^{\circ} & 48.5\end{array}$ $\begin{array}{lllll}50.9 & 50.8 & 50.7 & 50.0 & \text { - }\end{array}$ $\begin{array}{lllll}51.3 & 51.2 & 50.9 & 49.9 & 49.0\end{array}$ 10 49.0 50.7 49.0

$49.3 \quad 49.4 \quad 47.6$ $\begin{array}{lllll}50.1 & 50.0 & 49.8 & 50.0 & 49.0\end{array}$ $\begin{array}{lllll}48.4 & 48.3 & 48.3 & 49.0 & -\end{array}$ $\begin{array}{lllll}49.6 & 49.6 & 49.2 & 49.2 & 48.7\end{array}$ $\begin{array}{lllll}49.2 & 49.1 & 49.0 & 48.6 & 47.8\end{array}$

7 - 7 - - -

9 48.$$
-
$$$$
-\quad-
$$$$
-
$$

$$
-
$$

$\begin{array}{lll}-1 & -1 \\ -12 & -1\end{array}$ $46.2 \quad 46.2$

\section{$47.1 \quad 47.4$} 46.3 47.0

47.3 $\begin{array}{ccccc}- & - & - & - & -\end{array}$ 44.5 45.4 45.5 46.4 $-$
Salinity $\%$

$0^{\prime} \quad 150^{\prime}$

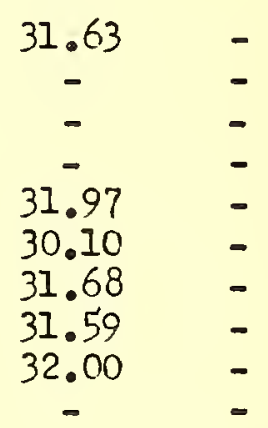

$.11 \quad 32.19$
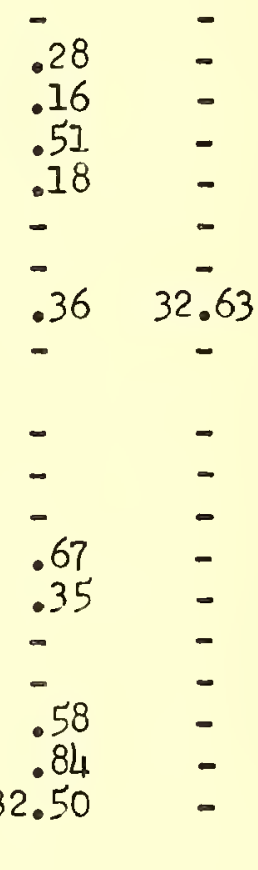

$32.09 \quad 32.41$ 
PORTLAND LIGHTSHIP $43^{\circ} 31.6^{\prime} \mathrm{N} .70^{\circ} 05.5^{\prime} \mathrm{W}$. Depth of water - 150 feet

\section{December 1956}

\section{Temperature of}

Day $0^{\prime} 30^{\prime} 50^{\prime} 100^{\prime} 150^{\prime}$

$\begin{array}{llllll}1 & 44.3 & 44.9 & 45.0 & 45.0 & 45.7\end{array}$

$\begin{array}{llllll}2 & 44.2 & 45.1 & 45.3 & 46.0 & 46.3\end{array}$

3 - $\quad$ - $\quad$ - $\quad$ -

$\begin{array}{llllll}4 & 4.0 & - & - & - & - \\ 6 & -2 & 4.3 & 45.0 & 45.5\end{array}$

$\begin{array}{llllll}6 & 45.0 & 45.0 & 45.0 & 45.7 & 45^{-}\end{array}$

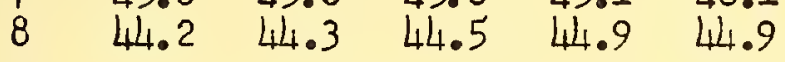

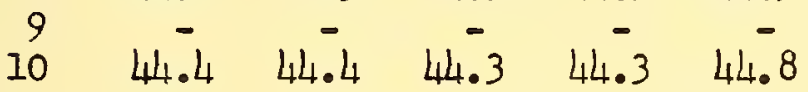

11
12

13

14

15

$\begin{array}{llllll}17 & 43.5 & 43.9 & 43.9 & 44.0 & 44.0\end{array}$

18

19

20

$\begin{array}{llllll}21 & 43.5 & 43.7 & 43.9 & 44.0 & 44.0\end{array}$

22 - $\quad-\quad-\quad-\quad$ -

23

24

26

27
28

29

30

31

$\begin{array}{lllll}43.8 & 43.8 & 43.9 & 45.0 & 45.8\end{array}$

$44.0 \quad 44.0 \quad 44.0 \quad 44.9 \quad 45.9$

$\begin{array}{lllll}43.9 & 43.9 & 43.9 & 44.5 & 45.1\end{array}$

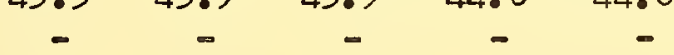

- $\quad-\quad-$

$\begin{array}{ccccc}- & - & - & - & -\end{array}$

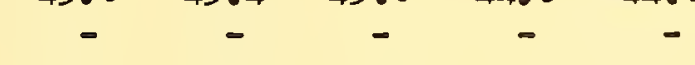

$\begin{array}{lllll}43.1 & 43.5 & 43.5 & 43.8 & 43.9\end{array}$

Mean
$43.9 \quad 44.2 \quad 44.3 \quad 44.7 \quad 45.7$

$32.38 \quad 32.66$
Salinity $\%$

$0^{\prime} 150^{\prime}$

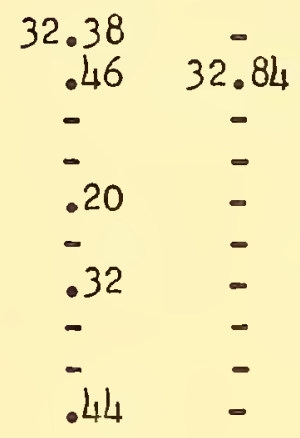

$.15 \quad 32.98$

$\begin{array}{cc}.30 & - \\ - & - \\ - & - \\ .55 & 32.39 \\ = & - \\ - & - \\ - & -\end{array}$

$.53 \quad-$

$-\quad-$

$.40 \quad 32.44$

$\begin{array}{ll}- & - \\ .48 & - \\ - & - \\ - & -\end{array}$ 
Mean surface temperatures for 1956 exhibited little departure from the means for the period 1925-1941 (Bumpus, 1957) except for the last of May and early June which were several degrees cooler and the last of July and first two-thirds of August which were several degrees warmer than the means for the period of record. The autumn temperatures cooled more slowly than the mean.

Water temperatures at this position ranged from slightly less than 370 , surface to bottom, in March, to $680 \mathrm{~F}$ at the surface in Ausust and almost $520 \mathrm{~F}$ at the bottom in October. The thermocline was well developed from mid-June to mid-September.

Surface salinity ranzed from $32.5 \%$ in January to $30.5 \%$ in mid-June with recovery to nearly $32.0 \%$ by the end of the year.

Bottom salinities were $1 / 2$ to $3 / 4 \% / 00$ higher than the surface salinities during the period of thermocline development, but generally tended to follow the surface salinity at each end of the year.
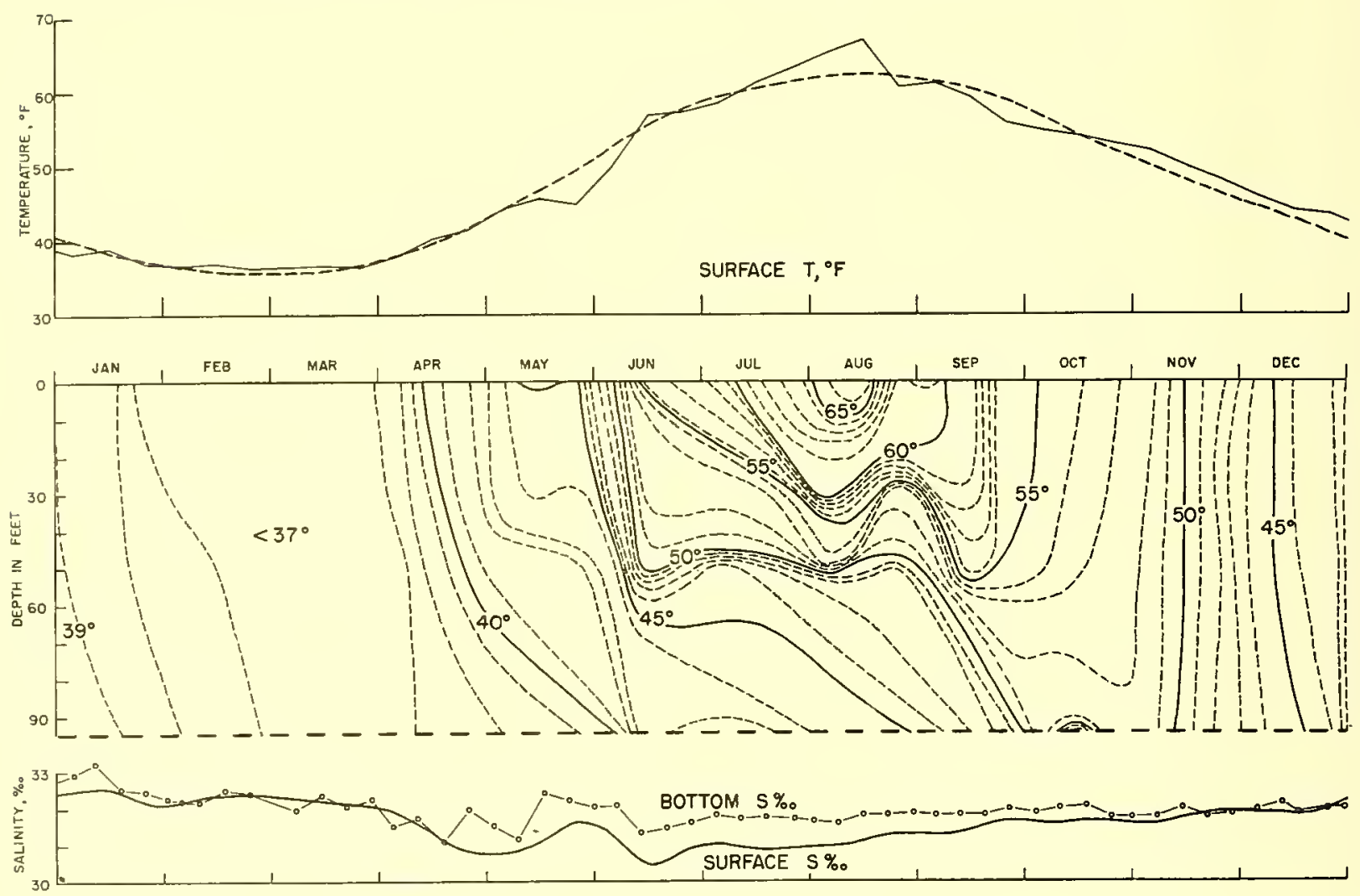

Figure 5. Boston Lightship, 1956. (Dashed line in upper diagram, mean for period $1025-1041)$ 
Table 4

BOSTON LIGHTSHIP

1955

November

Temperature F
Salinity $/ 00$

$0^{\prime} \quad 95^{\prime}$

Depth

Das

1

2

3

4

6

7
8

8

10

11

12

$\begin{array}{lllll}13 & 50.2 & 50.2 & 50.3 & 51.0\end{array}$

$\begin{array}{lllll}14 & 50.0 & 50.2 & 50.3 & 50.9\end{array}$

$\begin{array}{lllll}15 & 50.2 & 50.1 & 50.2 & 50.4\end{array}$

$\begin{array}{lllll}16 & 49.9 & 49.9 & 50.1 & 50.4\end{array}$

$\begin{array}{lllll}17 & 48.9 & 49.5 & 49.7 & 49.4\end{array}$

$\begin{array}{lllll}18 & 49.3 & 49.2 & 49.2 & 49.2\end{array}$

$\begin{array}{lllll}19 & 47.5 & 49.0 & 49.2 & 49.2\end{array}$

20

21 22 23

$48.5 \quad 48.5 \quad 48.5 \quad 48.5$

$24 \quad 48.0 \quad 48.0 \quad 48.0 \quad 48.0$

25. $48.4 \quad 48.5 \quad 48.5 \quad 48.6$

$\begin{array}{lllll}26 & 47.4 & 47.9 & 47.9 & 47.9\end{array}$

$\begin{array}{lllll}27 & 46.9 & 46.9 & 46.9 & 46.9\end{array}$

$\begin{array}{llllll}28 & 45.4 & 47.8 & 48.0 & 48.0\end{array}$

$\begin{array}{lllll}29 & 47.2 & 47.2 & 47.2 & 47.1\end{array}$

30

31.

\section{$\frac{\text { December }}{0}$}

Temperature $\mathrm{F}$
Salinity $\stackrel{0}{100}$

$0^{\prime} \quad 30^{\prime} 50^{\prime} 95^{\prime} 0^{\prime} 95^{\prime}$

$\begin{array}{lllll}47.0 & 47.0 & 46.9 & 46.9 & 31.93\end{array}$

$\begin{array}{llllll}46.9 & 46.9 & 46.9 & 46.9 & .95 & -\end{array}$

$\begin{array}{lllll}46.4 & 46.3 & 46.2 & 46.6 & 27.01\end{array}$

$\begin{array}{lllll}45.1 & 45.1 & 45.1 & 45.5 & 31.80\end{array}$

$\begin{array}{lllll}45.1 & 45.1 & 45.2 & 45.7 & .82\end{array}$

$\begin{array}{lllll}45.0 & 45.0 & 45.0 & 45.0 & .86\end{array}$

$\begin{array}{llllll}44.2 & 44.1 & 44.1 & 44.1 & .86 & = \\ 45.7 & 45.7 & 45.7 & 45.7 & .92 & =\end{array}$

$\begin{array}{rrrrr}44.9 & 45.0 & 45.0 & 45.9 & .93 \\ 44.0 & 44.0 & 44.0 & 44.0 & 31.91\end{array}$

$\begin{array}{lllll}44.7 & 44.7 & 44.7 & 44.7 & 32.01\end{array}$

$\begin{array}{rrrrr}44.9 & 44.9 & 44.9 & 44.9 & .03\end{array}$

$44.7 \quad 44.7 \quad 44.8 \quad 44.9 \quad .12=$

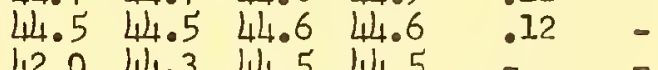

$\begin{array}{llllll}42.0 & 44.3 & 44.5 & 44.5 & - & - \\ 43.2 & 43.2 & 43.2 & 43.2 & 32.08 & -\end{array}$

$\begin{array}{lllll}42.9 & 42.9 & 42.9 & 43.2 & 30.79\end{array}$

$\begin{array}{lllll}42.4 & 42.4 & 42.3 & 44.0 & 32.02\end{array}$

$42.0 \quad 42.0 \quad 42.0 \quad 44.0 \quad .00 \quad-$

$41.441 .4 \quad 41.4 \quad 41.4 \quad .07 \quad-$

$\begin{array}{llllll}40.1 & 40.1 & 40.1 & 39.9 & .06 & 32.06 .\end{array}$

$\begin{array}{llllllll}39.9 & 40.1 & 40.1 & 40.0 & .18 & \text { - }\end{array}$

$\begin{array}{lllll}39.2 & 39.3 & 39.3 & 39.6 & .18\end{array}$

$\begin{array}{llllll}39.0 & 39.1 & 39.2 & 41.0 & .21 & -\end{array}$

$\begin{array}{llllll}39.3 & 39.8 & 41.0 & 41.0 & .24 & -\end{array}$

$\begin{array}{lllll}39.7 & 40.0 & 40.0 & 40.0 & .36\end{array}$

$\begin{array}{llllll}39.6 & 40.0 & 40.0 & 40.0 & .46 & -\end{array}$

$\begin{array}{llllll}40.1 & 40.1 & 40.1 & 40.0 & .56 & 32.67\end{array}$

$\begin{array}{llllll}39.7 & 39.9 & 40.0 & 40.2 & .47 & =\end{array}$

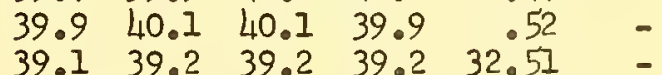

$\begin{array}{lllllllllllll}\text { Meant } & 48.3 & 48.7 & 48.7 & 48.8 & 31.71 & - & 42.7 & 42.8 & 42.8 & 43.1 & 31.89 & 32.36\end{array}$ 


\section{6}

Temperature $\mathrm{F}$

Depth

Day $0^{\prime} \quad 30^{\prime} 50^{\prime} \quad 95^{\prime} \quad 0^{\prime} \quad 95$

$\begin{array}{llllll}1 & 39.2 & 39.3 & 39.4 & 39.2 & 32.52\end{array}$

$\begin{array}{lllll}2 & 39.0 & 39.0 & 39.0 & 38.7\end{array}$

$\begin{array}{lllll}3 & 38.8 & 38.9 & 38.9 & 38.8\end{array}$

$\begin{array}{llllll}4 & 38.0 & 38.0 & 38.0 & 40.1\end{array}$

$\begin{array}{lllll}5 & 38.8 & 38.8 & 38.8 & 39.0\end{array}$

$\begin{array}{llllll}6 & 38.6 & 38.6 & 38.6 & 38.6\end{array}$

$\begin{array}{lllll}7 & 38.2 & 38.3 & 38.3 & 38.3\end{array}$

$\begin{array}{lllll}8 & 37.5 & 38.1 & 38.1 & 38.2\end{array}$

$\begin{array}{lllll}9 & 37.7 & 37.7 & 37.7 & 37.7\end{array}$

$\begin{array}{lllll}10 & 36.4 & 36.8 & 36.8 & 36.8\end{array}$

$\begin{array}{llllll}11 & 39.7 & 39.9 & 40.0 & 40.9\end{array}$

$\begin{array}{lllll}12 & 38.7 & 39.1 & 39.1 & 39.1\end{array}$

$\begin{array}{lllll}13 & 39.1 & 39.1 & 39.1 & 39.1\end{array}$

$\begin{array}{lllll}14 & 38.8 & 39.0 & 39.0 & 39.1\end{array}$

$\begin{array}{lllll}15 & 38.9 & 39.0 & 39.0 & 39.1\end{array}$

$\begin{array}{lllll}16 & 38.8 & 38.9 & 39.0 & 39.1\end{array}$

$\begin{array}{lllll}17 & 38.9 & 39.0 & 39.1 & 39.2\end{array}$

$\begin{array}{lllll}18 & 38.7 & 38.8 & 38.8 & 38.8\end{array}$

$\begin{array}{lllll}19 & 38.3 & 38.5 & 38.5 & 38.7\end{array}$

$\begin{array}{lllll}20 & 37.8 & 38.2 & 38.2 & 38.8\end{array}$

$\begin{array}{llllll}21 & 37.7 & 38.2 & 38.2 & 38.3\end{array}$

$\begin{array}{llllll}22 & 37.9 & 37.9 & 38.1 & 38.3\end{array}$

$\begin{array}{lllll}23 & 37.2 & 37.6 & 38.0 & 38.9\end{array}$

$\begin{array}{lllll}24 & 36.7 & 38.0 & 38.1 & 39.0\end{array}$

$\begin{array}{lllll}25 & 36.6 & 37.0 & 37.0 & 38.0\end{array}$

$\begin{array}{lllll}26 & 36.3 & 36.7 & 36.8 & 37.9\end{array}$

$\begin{array}{llllll}27 & 36.7 & 37.0 & 37.1 & 38.0\end{array}$

$\begin{array}{lllll}28 & 36.7 & 37.1 & 37.1 & 38.1\end{array}$

$\begin{array}{lllll}29 & 36.7 & 37.1 & 37.2 & 37.8\end{array}$

$\begin{array}{lllll}30 & 37.1 & 37.2 & 37.5 & 38.9\end{array}$

$\begin{array}{lllll}31 & 36.5 & 37.1 & 37.1 & 37.3\end{array}$

\section{February}

Salinity $/ 00$ Temperature F
Salinity /oo

$0^{\prime} \quad 30^{\prime} \quad 50^{\prime} 95^{\prime} \quad 0^{\prime} 95^{\prime}$

$\begin{array}{llllll}36.9 & 37.0 & 37.0 & 37.5 & 32.19 & 32.25\end{array}$

$\begin{array}{lllll}36.8 & 37.0 & 37.1 & 37.8\end{array}$

$\begin{array}{llll}36.5 & 36.6 & 36.8 & 39.1\end{array}$

$\begin{array}{llll}37.0 & 36.8 & 36.8 & 39.0\end{array}$

$\begin{array}{llll}37.0 & 37.0 & 37.1 & 38.4\end{array}$

$\begin{array}{llll}36.6 & 37.0 & 37.0 & 37.5\end{array}$

$\begin{array}{llll}36.0 & 36.5 & 37.1 & 38.0\end{array}$

$\begin{array}{llll}37.0 & 37.0 & 37.0 & 37.8\end{array}$

$\begin{array}{llll}36.1 & 36.5 & 36.6 & 37.2\end{array}$

$\begin{array}{llll}36.5 & 37.0 & 37.0 & 37.3\end{array}$

$\begin{array}{llll}36.8 & 37.0 & 37.0 & 37.3\end{array}$

$\begin{array}{llll}36.9 & 36.5 & 37.0 & 37.1\end{array}$

$\begin{array}{llll}36.7 & 36.6 & 36.6 & 36.6\end{array}$

$\begin{array}{llll}37.0 & 37.0 & 37.0 & 37.1\end{array}$

$37.0 \quad 37.0 \quad 37.0 \quad 37.1$

$36.7 \quad 37.1 \quad 37.1 \quad 37.1$

$\begin{array}{llll}36.0 & 37.0 & 37.0 & 37.0\end{array}$

$37.0 \quad 37.0 \quad 37.0 \quad 37.5$

$\begin{array}{llll}36.9 & 37.0 & 37.0 & 37.2\end{array}$

$\begin{array}{llll}37.0 & 37.0 & 37.1 & 37.8\end{array}$

$\begin{array}{llll}36.7 & 37.0 & 37.0 & 38.9\end{array}$

$36.0 \quad 37.0 \quad 37.0 \quad 38.0$

$\begin{array}{llll}35.8 & 36.6 & 36.6 & 36.8\end{array}$

$35.8 \quad 36.0$

36.036 .2

$\begin{array}{lllll}36.0 & 35.9 & 36.5 & 37.1 & 31.96\end{array}$

$\begin{array}{lllll}36.5 & 36.8 & 36.9 & 36.9 & 32.37\end{array}$

$\begin{array}{lllll}36.4 & 36.9 & 36.9 & 37.0\end{array}$

$\begin{array}{llll}36.2 & 36.8 & 37.0 & 37.0\end{array}$

$\begin{array}{llll}36.0 & 36.0 & 36.0 & 36.0\end{array}$

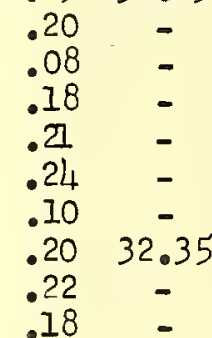

.56 . -

$.20=$

$.70:-$

$.05 \quad 32.77$

.4632 .17

.37

$.35 \quad-$

$.31 \quad-$

$\begin{array}{cc}.32 & - \\ .45 & 32.50 \\ .42 & - \\ 32.40 & - \\ 31.96 & - \\ 32.37 & = \\ .39 & = \\ .41 & = \\ .43 & 32.42\end{array}$

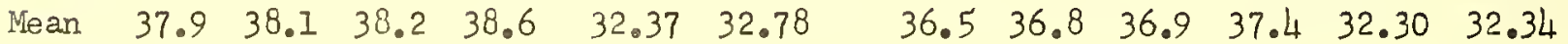




\section{6}

March

April

Temperature ${ }^{\circ} \mathrm{F}$

Salinity 100

Temperature $\mathrm{F}$

Salinity $/ 00$

Depth

Day $0^{\prime} \quad 30^{\prime} \quad 50^{\prime} \quad 95^{\prime} \quad 0^{\prime} \quad 95^{\prime} \quad 0^{\prime} \quad 30^{\prime} \quad 50^{\prime} \quad 95^{\prime} \quad 0^{\prime} \quad 95^{\prime}$

$1 \quad 36.7 \quad 36.7 \quad 36.6 \quad-\quad 32.47 \quad-$

$\begin{array}{lllll}36.5 & 36.2 & 36.1 & 36.3 & 31.92\end{array}$

$2 \quad 35.5 \quad 35.5 \quad 35.8 \quad 36.4$

$\begin{array}{llllll}36.0 & 36.0 & 36.0 & 37.0\end{array}$

.15

$.16-$

$\begin{array}{lllll}4 & 36.8 & 36.8 & 36.8 & 37.4\end{array}$

$\begin{array}{lllll}5 & 37.0 & 36.7 & 37.0 & 37.0\end{array}$

$\begin{array}{lllll}6 & 36.5 & 37.0 & 37.0 & 37.0\end{array}$

$\begin{array}{lllll}7 & 36.8 & 37.0 & 37.0 & 37.1\end{array}$

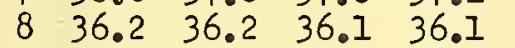

$\begin{array}{lllll}9 & 36.7 & 36.9 & 37.0 & 36.9\end{array}$

$\begin{array}{lllll}10 & 36.8 & 36.7 & 36.9 & 36.9\end{array}$

$.21 \quad-$

.38

32.28

$31.96 \quad 31.99$

32.44

.36

.31

$\begin{array}{lllll}17 & 37.0 & 37.0 & 37.2 & 37.2\end{array}$

$\begin{array}{lllll}12 & 37.0 & 37.1 & 37.1 & 37.2\end{array}$

$\begin{array}{lllll}13 & 36.6 & 36.8 & 37.0 & 37.0\end{array}$

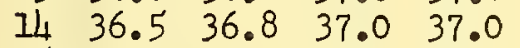

$\begin{array}{lllll}15 & 37.0 & 37.0 & 36.9 & 37.0\end{array}$

$\begin{array}{lllll}16 & 37.2 & 37.2 & 37.1 & 37.3\end{array}$

$\begin{array}{lllll}17 & 36.7 & 36.8 & 36.9 & 37.0\end{array}$

$\begin{array}{lllll}18 & 36.1 & 36.6 & 36.6 & 36.7\end{array}$

$\begin{array}{lllll}19 & 36.2 & 37.0 & 36.9 & 37.0\end{array}$

$\begin{array}{lllll}20 & 36.0 & 36.0 & 36.0 & 35.8\end{array}$

$21 \quad 36.4 \quad 36.2 \quad 36.0 \quad 36.8$

$22 \quad 36.8 \quad 36.8 \quad 37.0 \quad 37.0$

$\begin{array}{lllll}23 & 36.5 & 36.4 & 36.3 & 36.3\end{array}$

$\begin{array}{lllll}24 & 36.0 & 36.5 & 36.8 & 36.8\end{array}$

$\begin{array}{lllll}25 & 36.0 & 36.5 & 36.5 & 36.5\end{array}$

$\begin{array}{lllll}26 & 36.2 & 36.2 & 36.2 & 36.3\end{array}$

$\begin{array}{lllll}27 & 36.7 & 36.7 & 36.7 & 36.9\end{array}$

$\begin{array}{lllll}28 & 36.7 & 36.8 & 36.8 & 36.8\end{array}$

$\begin{array}{lllll}29 & 37.2 & 37.0 & 37.0 & 37.0\end{array}$

$\begin{array}{lllll}30 & 36.0 & 36.0 & 36.1 & 36.2\end{array}$

$\begin{array}{lllll}31 & 36.0 & 36.0 & 36.0 & 36.1\end{array}$

$\begin{array}{cc}.36 & - \\ .27 & - \\ .23 & - \\ .00 & 32.37 \\ .38 & - \\ .38 & - \\ .28 & - \\ .17 & - \\ 32.10 & - \\ 31.87 & -\end{array}$

$\begin{array}{cc}.90 & 32.07\end{array}$

$32.18=$

$\begin{array}{ll}.27 & - \\ .12 & -\end{array}$

.24

.22

.07

.08

32.03

31.90

32.24

$-$

$-$ $\begin{array}{llll}37.2 & 36.6 & 36.2 & 36.3 \\ 37.2 & 37.0 & 36.6 & 36.5\end{array}$

$\begin{array}{llllll}37.2 & 37.0 & 36.6 & 36.5 & 31.77\end{array}$

$\begin{array}{lllll}37.7 & 37.0 & 36.7 & 36.3 & 32.16\end{array}$

$\begin{array}{lllll}37.3 & 37.4 & 36.9 & 36.3 & 31.80\end{array}$

$\begin{array}{lllll}42.0 & 37.8 & 36.9 & 36.3 & .72\end{array}$

$\begin{array}{lllll}38.1 & 38.1 & 37.9 & 36.4 & .85\end{array}$

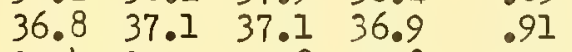

$\begin{array}{lllll}37.4 & 37.7 & 37.8 & 37.8 & .79\end{array}$

$\begin{array}{lllll}39.1 & 37.1 & 37.0 & 37.1 & .76\end{array}$

$39.4 \quad 37.9 \quad 37.9 \quad 37.1$

$\begin{array}{llll}38.7 & 38.5 & 38.3 & 37.2\end{array}$

$\begin{array}{llll}39.3 & 38.3 & 37.9 & 37.1\end{array}$

$\begin{array}{llll}39.3 & 38.3 & 38.2 & 37.9\end{array}$

$40.1 \quad 38.3$

$39.9 \quad 39.8$

$40.9 \quad 40.1$

39.0

37.5

$\begin{array}{cc}.72 & 31.74 \\ .78 & - \\ .48 & = \\ 31.35 & - \\ 30.91 & = \\ 31.26 & = \\ .12 & - \\ .27 & 31.10 \\ .28 & = \\ .28 & =\end{array}$

$\begin{array}{ll}40.4 & 39.1 \\ 47.7 & 39.4\end{array}$

38.

41.8

40.3

$\begin{array}{llll}40.9 & 40.1 & 39.6 & 37.1\end{array}$

.30
31.24

$41.2 \quad 41.0$

$\begin{array}{ll}40.5 & 40.7 \\ 40.7 & 40.9\end{array}$

$41.3 \quad 40.8$

37.2
40.7

30.85

$41.2 \quad 41.1$

40.8

39.9

41.3

$41.7 \quad 41.3$

$\begin{array}{ll}42.4 & 47.9 \\ 42.5 & 42.5\end{array}$

31.51

$-$

$-$

$-$

$\begin{array}{lllllll}\text { Mean } & 36.5 & 36.6 & 36.7 & 36.8 & 32.18 & 32.17\end{array}$

$\begin{array}{llllll}39.8 & 39.1 & 38.6 & 37.0 & 31.35 & 31.60\end{array}$ 
Table 4 (cont'd)

BOSTON LIGHTSHIP
Depth of water - 96 feet
1956

May

Temperature $\stackrel{\circ}{F}$

Salinity $\stackrel{\circ}{\circ}$
4220.4 N. $7045.5 \mathrm{w}$.
Temperature $\stackrel{\circ}{F} \quad$ Saline $^{\circ} \stackrel{\circ}{100}$

Temperature $\stackrel{\circ}{F} \quad$ Saline $^{\circ} \stackrel{\circ}{100}$

Depth

Da

$\begin{array}{llllll}1 & 44.0 & 43.0 & 41.7 & 37.6\end{array}$

$\begin{array}{lllll}2 & 44.3 & 43.3 & 42.8 & 38.2\end{array}$

$\begin{array}{lllll}3 & 44.4 & 43.5 & 39.6 & 38.0\end{array}$

$\begin{array}{lllll}4 & 44.9 & 43.7 & 40.2 & 38.0 \\ 5 & 44.1 & 43.9 & 41.2 & 38.1\end{array}$

$\begin{array}{lllll}6 & 43.2 & 43.5 & 42.0 & 37.7\end{array}$

$\begin{array}{lllll}7 & 43.4 & 43.1 & 40.8 & 38.0\end{array}$

$\begin{array}{lllll}8 & 44.0 & 43.8 & 43.5 & 38.1\end{array}$

$9 \quad 44.0 \quad 43.8 \quad 41.0 \quad 38.1$

$\begin{array}{lllll}10 & 44.0 & 43.9 & 39.9 & 38.1\end{array}$

$\begin{array}{lllll}11 & 45.3 & 44.0 & 40.0 & 38.0\end{array}$

$\begin{array}{lllll}12 & 45.6 & 44.0 & 40.6 & 38.0\end{array}$

$\begin{array}{lllll}13 & 46.5 & 44.3 & 39.8 & 38.1\end{array}$

$\begin{array}{lllll}14 & 45.6 & 45.5 & 42.2 & 38.7\end{array}$

$\begin{array}{lllll}15 & 45.7 & 45.2 & 40.3 & 38.5\end{array}$

$\begin{array}{lllll}16 & 46.0 & 45.3 & 42.7 & 38.6\end{array}$

$\begin{array}{lllll}17 & 45.2 & 44.9 & 44.8 & 38.8\end{array}$

$\begin{array}{lllll}18 & 44.5 & 44.1 & 41.5 & 38.0\end{array}$

$\begin{array}{lllll}19 & 45.0 & 44.5 & 39.5 & 38.0\end{array}$

$\begin{array}{lllll}20 & 46.1 & 45.9 & 40.4 & 38.1\end{array}$

$0^{\prime} \quad 95^{\prime}$

$0^{\prime} 30^{\prime} 50^{\prime} 95^{\prime} 0^{\prime}$

951

$\begin{array}{cc}30.69 & - \\ .75 & 31.53 \\ .75 & - \\ .81 & - \\ .79 & - \\ .85 & - \\ .74 & - \\ .87 & - \\ .91 & 31.19\end{array}$

$\begin{array}{lllll}48.2 & 44.4 & 41.8 & 39.0 & 31.71\end{array}$

$\begin{array}{lllll}48.7 & 47.1 & 43.1 & 39.0 & .59\end{array}$

$\begin{array}{lllll}49.0 & 46.2 & 40.5 & 38.9 & .57\end{array}$

$\begin{array}{llll}50.6 & 46.9 & 39.8 & 39.0\end{array}$

$\begin{array}{llll}49.5 & 47.1 & 41.8 & 38.8\end{array}$

$\begin{array}{llll}50.2 & 46.4 & 40.9 & 38.1\end{array}$

$51.1 \quad 45.5 \quad 42.6 \quad 38.2$

$\begin{array}{llll}50.8 & 49.3 & 43.3 & 39.0\end{array}$

$\begin{array}{lllll}50.2 & 50.1 & 47.9 & 40.2 & 31.08\end{array}$

.01

.0132 .10

.71

$51.0 \quad 50.7 \quad 49.0 \quad 44.4 \quad 30.64$

$.68-$

$\begin{array}{llll}51.7 & 50.4 & 49.7 & 43.4\end{array}$

$\begin{array}{llll}52.7 & 50.8 & 49.7 & 44.0\end{array}$

$\begin{array}{llll}56.1 & 51.1 & 50.5 & 45.0\end{array}$

$.80=$
$30.83=$
$31.07=$

$.13 \quad 32.42$

$56.3 \quad 51.3 \quad 50.6$

$58.8 \quad 52.9 \quad 51.5$

$63.6 \quad 55.7 \quad 51.54 .5$

$\begin{array}{llll}63.6 & 55.7 & 52.7 & 41.5\end{array}$

$56.1 \quad 55.9 \quad 53.8 \quad 46.0$

.62

.55

. 31 31. 33

$.08 \quad-$

$.15=$

$\begin{array}{llllll}27 & 45.8 & 44.0 & 41.7 & 38.0\end{array}$

$22 \quad 46.0 \quad 44.4 \quad 39.0 \quad 38.1$

$\begin{array}{lllll}23 & 43.2 & 42.8 & 41.9 & 38.1\end{array}$

$\begin{array}{lllll}24 & 45.2 & 43.8 & 40.8 & 38.9\end{array}$

$\begin{array}{lllll}25 & 45.6 & 43.6 & 42.7 & 38.9\end{array}$

$\begin{array}{lllll}26 & 44.0 & 43.6 & 42.7 & 38.5\end{array}$

$\begin{array}{lllll}27 & 44.9 & 44.5 & 42.7 & 38.5\end{array}$

$\begin{array}{lllll}28 & 43.5 & 41.7 & 40.2 & 39.0\end{array}$

$\begin{array}{lllll}29 & 45.0 & 43.4 & 42.1 & 38.5\end{array}$

$3044.1 \quad 44.1 \quad 43.7$ -

$\begin{array}{llll}31 & 46.6 & 43.2 & 41.3\end{array}$

$\begin{array}{llll}56.1 & 55.9 & 53.8 & 46.0\end{array}$

$\begin{array}{llll}56.5 & - & - & -\end{array}$

.44

.37

- 50

.23

.43

.48

$\begin{array}{llll}56.4 & 55.6 & 48.5 \quad 44.3\end{array}$

$\begin{array}{llll}56.1 & 54.1 & 48.2 \quad 43.3\end{array}$

$54.9 \quad 50.1 \quad 47.3 \quad 42.8$

$\begin{array}{llll}56.6 & 52.5 & 50.2 & 41.5\end{array}$

.62

$.33 \quad-.66$

$\begin{array}{ll}.66 & 32.22 \\ .55 & - \\ .74 & -\end{array}$

$57.3 \quad 56.1 \quad 50.6$

$\begin{array}{llll}59.3 & 53.1 & 47.4 & 40.8\end{array}$

$\begin{array}{llll}57.4 & 51.9 & 48.2 & 40.2\end{array}$

.64

30.62

31.16

.67 -

$.63-$

$58.9 \quad 53.1 \quad 46.9$

30.72

$.78=$

$.76 \quad-$

$.76 \quad 32.06$

$\begin{array}{llll}56.8 & 50.8 & 45.3 & 40.8\end{array}$

$58.2 \quad 48.3$

43.3

41.0

.69
.82

.8231 .63

31.73

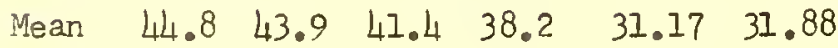

$54.7 \quad 50.8 \quad 47.0 \quad 47.5 \quad 30.83 \quad 31.64$ 
BOSTON LIGHTSHIP

\section{6}

\section{July}

Temperature F

\author{
$\frac{\text { August }}{0}$

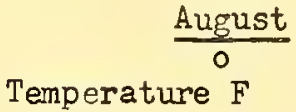

Salinity 100

Depth

Day $0^{\prime} \quad 30^{\prime} 50^{\prime} 95^{\prime} \quad 0^{\prime} \quad 95^{\prime}$

$\begin{array}{llllll}1 & 60.3 & 48.0 & 44.3 & 41.2 & 30.93\end{array}$

Salinity $/ 00$

$0^{\prime} 30^{\prime} 50^{\prime} 95^{\prime} 0^{\prime}$

$95^{\prime}$

$\begin{array}{lllll}2 & 56.7 & 50.0 & 43.2 & 41.4\end{array}$

$\begin{array}{lllll}3 & 59.0 & 54.2 & 43.9 & 41.8\end{array}$

$\begin{array}{lllll}4 & 60.5 & 5.5 & 46.0 & 47.5\end{array}$

$\begin{array}{lllll}5 & 60.8 & 54.9 & 46.2 & 47.9\end{array}$

$\begin{array}{lllll}6 & 57.1 & 55.8 & 47.6 & 42.1\end{array}$

$\begin{array}{lllll}7 & 56.6 & 53.9 & 48.3 & 42.8\end{array}$

$\begin{array}{lllll}8 & 59.1 & 57.2 & 49.4 & 42.2\end{array}$

$\begin{array}{lllll}9 & 56.7 & 48.4 & 43.2 & 42.4\end{array}$

$\begin{array}{lllll}10 & 58.6 & 46.9 & 43.7 & 42.2\end{array}$

$\begin{array}{lllll}11 & 60.9 & 49.2 & 43.2 & 42.8\end{array}$

$\begin{array}{lllll}12 & 60.9 & 50.0 & 45.6 & 42.4\end{array}$

$\begin{array}{lllll}13 & 62.9 & 57.5 & 48.4 & 42.9\end{array}$

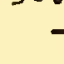

.99

.9831 .84

.97

.96

.98

30.97

31.02

31.

$30.91 \quad 31.72$

$.85 \quad-$
$.87=$

.91

$\begin{array}{llllll}15 & 61.5 & 53.9 & 49.2 & 42.7 & .89\end{array}$

$\begin{array}{lllll}16 & 61.1 & 50.3 & 45.0 & 41.8\end{array}$

$\begin{array}{llllll}17 & 60.5 & 5.3 & 45.7 & 42.0\end{array}$

$\begin{array}{lllll}18 & 60.7 & 52.9 & 45.1 & 47.9\end{array}$

$\begin{array}{lllll}19 & 61.3 & 55.0 & 46.6 & 41.9\end{array}$

$20 \quad 61.8 \quad 54.5 \quad 44.9 \quad 42.1$

$\begin{array}{llllll}21 & 62.1 & 55.6 & 46.0 & 42.4\end{array}$

$\begin{array}{lllll}22 & 59.3 & 47.9 & 45.0 & 42.9\end{array}$

$\begin{array}{lllll}23 & 59.8 & 53.9 & 47.3 & 42.8\end{array}$

$\begin{array}{lllll}24 & 61.4 & 52.2 & 45.8 & 43.1\end{array}$

$\begin{array}{lllll}25 & 62.3 & 53.3 & 46.2 & 42.9\end{array}$

$\begin{array}{lllll}26 & 64.9 & 54.0 & 46.7 & 43.1\end{array}$

$\begin{array}{lllll}27 & 63.3 & 52.5 & 46.9 & 43.7\end{array}$

$\begin{array}{lllll}28 & 65.7 & 60.2 & 50.5 & 43.5\end{array}$

$\begin{array}{lllll}29 & 64.7 & 56.0 & 46.3 & 43.5\end{array}$

$\begin{array}{lllll}30 & 65.1 & 54.7 & 49.0 & 43.9\end{array}$

$\begin{array}{lllll}31 & 65.5 & 63.0 & 49.3 & 43.9\end{array}$
$.87 \quad-$

.8931 .78

-

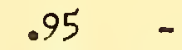

\section{.97}

.97

.92

$.93-$

30.95

31.01

30.9

.2

30.74

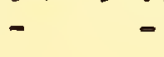

$\begin{array}{lllllll}\text { Mean } \quad 61.0 & 53.3 & 46.3 & 42.5 & 30.91 & 31.76\end{array}$

$\begin{array}{rrrrrc}64.6 & 57.6 & 49.3 & 43.8 & 30.79 & 31.66 \\ 65.1 & 63.9 & 54.2 & 43.9 & .75 & - \\ 65.7 & 56.7 & 48.1 & 44.2 & .81 & - \\ 65.8 & 60.6 & 50.3 & 43.9 & .89 & - \\ 65.8 & 57.1 & 49.9 & 43.8 & .92 & - \\ 65.4 & 53.9 & 50.6 & 44.0 & .91 & - \\ 64.0 & - & - & - & .98 & - \\ 64.1 & 63.3 & 57.1 & 46.2 & 30.97 & 31.60 \\ 66.9 & 65.2 & 64.7 & 46.1 & 31.05 & - \\ 66.9 & 66.1 & 58.8 & 43.8 & 31.05 & -\end{array}$

$\begin{array}{lllll}67.3 \quad 63.8 & 49.0 & 44.4 & 30.97 \quad-\end{array}$ $\begin{array}{lllll}68.0 & 60.2 & 52.2 & 44.2 \quad .98 & -\end{array}$

$\begin{array}{llllll}67.1 & 61.0 & 52.0 & 44.5 & 30.99 & -\end{array}$

$\begin{array}{lllll}67.7 & 56.1 & 49.9 & 45.1 & 31.03\end{array}$

$\begin{array}{llll}67.5 & 57.1 & 49.2 & 44.8\end{array}$

$\begin{array}{llll}66.9 & 52.2 & 46.4 & 44.8\end{array}$

$64.9 \quad 52.2 \quad 48.6 \quad 44.2$

$64.1 \quad 57.8 \quad 49.4 \quad 44.2$

$\begin{array}{llll}68.2 & 53.9 & 49.3 \quad 43.9\end{array}$

$\begin{array}{llll}68.1 & 55.2 \quad 47.9 \quad 44.5\end{array}$

.0331 .84

.05

$.10 \quad-$

$.08-$

$.10 \quad-$

$\begin{array}{llllll}66.3 & 53.8 & 50.6 & 44.8 & .05 & -\end{array}$

$63.0-0.722-$

$\begin{array}{llllll}63.0 & 51.1 & 46.2 & 44.3 & .22 & . \\ 61.2 & 51.3 & -\end{array}$

$\begin{array}{lllll}61.4 & 52.3 & 47.2 & 44.0 & .22\end{array}$

60.0 - $-\quad$ - .29 -

$60.5-0.36$ -

$\begin{array}{llllll}57.7 & 47.6 & 46.4 & 44.3 & .34 & - \\ 59.0 & 50.4 & 48.6 & 44.8 & .40 & 31.88\end{array}$

$\begin{array}{llll}58.1 & 49.9 & 46.7 & 45.1\end{array}$ $\begin{array}{llll}58.4 & 53.1 & 45.8 & 45.2\end{array}$
.48

.26

$\begin{array}{llllll}64.2 & 56.7 & 50.2 & 44.5 & 31.08 & 31.76\end{array}$ 


\section{6}

\section{September}

Temperature ${ }^{\circ} \mathrm{F}$
Salinity $\%$
October

Temperature ${ }^{\circ} \mathrm{F}$.
Salinity $\%$

Depth

Dayr

Day $0^{\prime} \quad 30^{\prime} 50^{\prime} 95^{\prime} \quad 0^{\prime} \quad 95$

$161.459 .649 .8-31.32$ -

$\begin{array}{lllllr}2 & 62.0 & 55.0 & 51.7 & 46.0 & .32\end{array}$

$\begin{array}{llllll}3 & 61.0 & 55.0 & 49.6 & 46.3 & .21\end{array}$

$\begin{array}{lllll}4 & 64.0 & 56.3 & 47.0 & 45.5\end{array}$

$\begin{array}{lllll}5 & 61.2 & 59.7 & 47.3 & 46.0\end{array}$

$\begin{array}{lllll}6 & 62.5 & 58.8 & 49.7 & 45.5\end{array}$

$\begin{array}{lllll}7 & 61.2 & 61.0 & 48.4 & 46.1\end{array}$

$.32-$

.2731 .80

$\begin{array}{lllll}8 & 60.0 & 59.7 & 55.5 & 46.0\end{array}$

31.30 -
30.90 -

$959.0 \quad 59.0 \quad 58.4$ -

$10 \quad 59.1 \quad 58.5 \quad 58.5$

31.20

.35

1

1

$\begin{array}{llll}59.0 & 58.3 \quad 58.2 \quad 48.7\end{array}$

$\begin{array}{llll}59.2 & 58.4 & 58.2 & 47.5\end{array}$

$\begin{array}{lll}58.4 & 58.2 & 47.5 \\ 58.3 & 57.2 & 47.7\end{array}$

1459.6

$59.3 \quad 57.8 \quad 47.0$

1560.0

$16 \quad 59.5$

$-\quad-$

$5 \overline{7} .5$

$59.5 \quad 57.5 \quad 46.5$

$\begin{array}{lllll}17 & 59.6 & 59.5 & 58.8 & 46.6 \\ 18 & 59.2 & 58.5 & 55.3 & 46.1\end{array}$

$\begin{array}{lllll}19 & 58.8 & 57.8 & 57.4 & 46.6\end{array}$

20

58.0

$\begin{array}{lll}57.9 & 50.6 \quad 46.1\end{array}$

2

22

22

2

2

25

27

27
28

29

29

31

$\begin{array}{llll}57.0 \quad 56.8 & 54.0 \quad 46.2\end{array}$

$\begin{array}{llll}57.0 & 56.4 & 56.2 & 45.8\end{array}$

$\begin{array}{llll}57.0 & 56.8 & 52.9 & 46.0\end{array}$

$\begin{array}{llll}56.6 & 56.2 \quad 50.2 \quad 45.1\end{array}$

$\begin{array}{llll}56.0 & 56.4 & 55.7 & \overline{1} \\ 55.6 & 55.4 & 55.0 & 46.8\end{array}$

$54.5 \quad 54.3 \quad 53.9 \quad 47.8$

54.2

$54.5 \quad 54.4 \quad 54.0 \quad 53.9$

$55.6 \quad 54.9 \quad 54.8 \quad 54.5$
.35

.3

$.38 \quad-$

31.82

.33

.31

.46

.46

.46

$.46 \quad 31.82$

.44

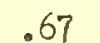

.77

.68

.56

.63

.56

.51

.58
.66

31.73

-

-

$\begin{array}{lllllll}\text { Mean } & 58.7 \quad 57.4 & 54.1 & 47.1 & 31.43 & 31.86\end{array}$

54.153 .7

$-$

$-$ $\begin{array}{ll}55.1 & 55.0 \\ 55.4 & 54.7 \\ 54.8 & 54.8 \\ 54.9 & 54.6 \\ 54.9 & 54.9 \\ 54.7 & 54.5 \\ 54.9 & 54.7 \\ 54.6 & 54.5 \\ 55.0 & 54.5 \\ 54.5 & 54.3\end{array}$

$54.0 \quad 53.9 \quad 53.9 \quad 47.4$

$\begin{array}{llll}53.5 & 53.2 & 53.2 & 48.5 \\ 54.0 & 53.0 & 53.0 & 48.2\end{array}$

$53.5 \quad 53.0 \quad 53.0 \quad 47.3$

$53.9 \quad 53.5 \quad 53.1 \quad 47.2$

$54.454 .1 \quad 53.1 \quad 47.2$

$\begin{array}{llll}56.1 & 55.5 \quad 54.0 & 46.8\end{array}$

$56.1 \quad 55.1 \quad 53.9 \quad 47.3$

$53.1 \quad 53.1 \quad 53.0 \quad 48.2$

$\begin{array}{llll}53.6 & 52.7 & 52.7 & 49.0\end{array}$

$54.0 \quad 53.0 \quad 53.0 \quad 49.7$

$\begin{array}{llll}53.5 & 53.1 & 52.5 & 50.0\end{array}$

$\begin{array}{llll}53.4 & 53.1 & 53.1 & 49.4\end{array}$

$\begin{array}{llll}53.8 & 53.8 & 53.5 & 50.8\end{array}$

$\begin{array}{llll}52.9 & 52.9 & 52.8 & 52.7\end{array}$

$52.4 \quad 52.3 \quad 52.3 \quad 52.3$

$53.0 \quad 52.8 \quad 52.5 \quad 52.5$

$52.9 \quad 52.8 \quad 52.8 \quad 52.5$

$\begin{array}{llll}53.0 & 52.3 & 52.3 & 52.2\end{array}$

$52.4 \quad 52.4 \quad 52.4 \quad 52.0$

53.3

52.3

$52.3 \quad 51.9$ $0^{\prime}$

951

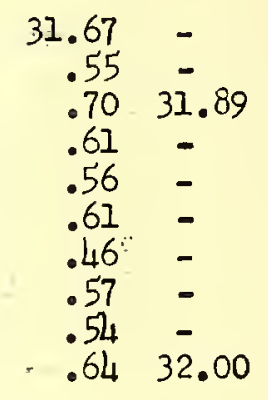

.09.

$.74-$

$.72-$

$.72-$

.63

$.56 \quad 32.06$

$.53-$

$.06-$

$\begin{array}{ll}.74 & - \\ .73 & - \\ .57 & - \\ .58 & 31.76 \\ .62 & - \\ .67 & - \\ .60 & - \\ .59 & - \\ .66 & - \\ .44 & - \\ .39 & 31.78\end{array}$


BOSTON LIGHTSIP

Depth of water - 96 feet

\section{6}

November

Temperature ${ }^{\mathrm{O}} \mathrm{F}$
Salinity $\%$

\section{December}

Temperature ${ }^{\circ} \mathrm{F}$ Salinity $\%$

Depth

Da

$\begin{array}{lllll}1 & 53.3 & 52.3 & 52.2 & 52.0 \\ 2 & 52.8 & 52.8 & 52.8 & 52.2\end{array}$

$\begin{array}{lllll}3 & 52.2 & 52.2 & 52.2 & 52.2\end{array}$

$\begin{array}{lllll}4 & 52.2 & 52.1 & 52.0 & 52.0\end{array}$

$\begin{array}{lllll}5 & 51.9 & 51.9 & 51.9 & 51.8\end{array}$

$\begin{array}{lllll}6 & 52.5 & 51.9 & 51.8 & 51.9\end{array}$

$\begin{array}{lllll}7 & 52.1 & 51.9 & 51.9 & 51.8\end{array}$

$\begin{array}{lllll}8 & 52.4 & 51.9 & 51.8 & 51.4\end{array}$

$\begin{array}{llllll}9 & 52.0 & 51.9 & 51.9 & 51.1\end{array}$

$\begin{array}{lllll}10 & 51.1 & 51.0 & 50.9 & 50.9\end{array}$

$\begin{array}{lllll}\text { II } & 50.4 & 50.3 & 50.3 & 50.1\end{array}$

$\begin{array}{llllll}12 & 50.8 & 50.7 & 50.7 & 49.2\end{array}$

$\begin{array}{lllll}13 & 50.2 & 50.2 & 50.2 & 48.9\end{array}$

$\begin{array}{lllll}14 & 49.9 & 49.9 & 49.9 & 49.8\end{array}$

$\begin{array}{llllll}15 & 50.3 & 49.7 & 49.9 & 49.9\end{array}$

$\begin{array}{lllll}16 & 50.1 & 50.1 & 50.1 & 49.5\end{array}$

$\begin{array}{lllll}17 & 50.0 & 50.0 & 50.0 & 48.3\end{array}$

$\begin{array}{lllll}18 & 49.0 & 49.8 & 40.8 & 49.4\end{array}$

$\begin{array}{llllll}19 & 49.7 & 49.6 & 49.5 & 49.3\end{array}$

$\begin{array}{lllll}20 & 49.2 & 49.2 & 49.2 & 49.5\end{array}$

$\begin{array}{llllll}21 & 49.7 & 49.7 & 49.7 & 49.8\end{array}$

$\begin{array}{llllll}22 & 49.7 & 49.7 & 49.7 & 48.7\end{array}$

$\begin{array}{llllll}23 & 48.9 & 48.9 & 48.9 & 48.0\end{array}$

$\begin{array}{llllll}24 & 48.7 & 48.7 & 48.7 & 48.7\end{array}$

$\begin{array}{lllll}25 & 48.6 & 48.5 & 48.4 & 48.0\end{array}$

$\begin{array}{llllll}26 & 48.0 & 47.0 & 46.9 & 46.9\end{array}$

$\begin{array}{llllll}27 & 46.5 & 46.7 & 46.7 & 47.2\end{array}$

$\begin{array}{lllll}28 & 46.5 & 46.5 & 46.6 & 46.8\end{array}$

$\begin{array}{lllll}29 & 47.3 & 46.2 & 47.2 & 47.4\end{array}$

$\begin{array}{lllll}30 & 47.5 & 47.5 & 47.5 & 47.5\end{array}$ 31 $0^{\prime} \quad 951$

31.28

.52

.67

.61

.73

.63

$\begin{array}{ll}.36 & 31.79\end{array}$

$.30 \quad-$

$.59 \quad-$

$.74 \ldots$

.74

$.71-$

$\begin{array}{ll}.88 & - \\ .92 & 32.01\end{array}$

$.77 \quad-$

$.80 \quad-$

$.77 \quad-$

$.74 \quad-$

$.74 \quad-$

.76

.83

.93

.94

.95

.83

.73

.82

$31.92=$

31.78

$-$

$-$

-

$-$

$-82$

$-$

Mean
$50.1 \quad 50.0 \quad 50.0 \quad 49.6$
31.73

31.85 o' $30^{\prime} 50^{\prime} \quad 95^{\prime}$ o' $95^{\circ}$

$\begin{array}{llllll}47.1 & 47.1 & 47.1 & 47.1 & 31.97 & -\end{array}$

$\begin{array}{llllll}46.9 & 46.9 & 46.9 & 47.1 & .96 & -\end{array}$

$\begin{array}{llllll}47.0 & 47.0 & 47.0 & 46.9 & .99 & -\end{array}$

$\begin{array}{llllll}46.7 & 46.6 & 46.5 & 46.6 & .99 & -\end{array}$

$\begin{array}{llllll}46.0 & 45.9 & 45.9 & 45.9 & .92 & 31.94\end{array}$

$\begin{array}{lllllll}45.3 & 45.6 & 45.9 & 46.8 & .80 & -\end{array}$

$\begin{array}{lllllll}45.0 & 45.0 & 45.0 & 47.1 & .71 & -\end{array}$

$\begin{array}{lllll}4.8 & 44.744 .9 & - & .70 & -\end{array}$

$\begin{array}{llllllll}45.1 & 45.1 & 45.1 & 46.2 & .77 & -\end{array}$

$\begin{array}{lllllll}45.1 & 45.1 & 45.2 & 45.8 & .91 & -\end{array}$

$\begin{array}{lllll}4.4 & 44.5 & 45.0 & 46.0 \quad & .78\end{array}$

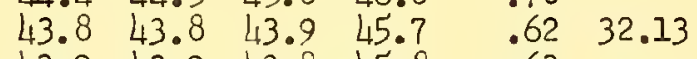

$\begin{array}{llllll}43.9 & 43.9 & 43.8 & 45.8 & .63 & -\end{array}$

$\begin{array}{lllllll}4.0 & 44.0 & 44.0 & 45.7 & .79 & -\end{array}$

$\begin{array}{lllll}44.8 & 44.6 & 44.7 \quad 44.8 & 31.87 & -\end{array}$

$\begin{array}{llllll}46.2 & 46.1 & 46.2 & 46.7 & .76 & 31.83\end{array}$

$\begin{array}{lllll}46.2 & 46.7 & 46.7 & 42.4 & .76\end{array}$

$\begin{array}{lllll}43.9 & 43.8 & 44.1 & 45.5 & .82\end{array}$

$41.0 \quad 47.0 \quad 47.0 \quad 47.8 \quad .86$

$\begin{array}{lllll}41.4 & 41.6 & 41.8 & 42.3 \quad .88 & =\end{array}$

$\begin{array}{lllllll}46.5 & 46.9 & 47.2 & 47.1 & .91 & -\end{array}$

$42.342 .342 .3-.95=$

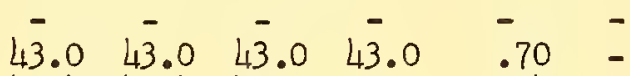

$47.447 .441 .5-94=$

$\begin{array}{llllll}40.1 & 40.1 & 40.2 & 40.9 & 31.92 & -\end{array}$

$\begin{array}{lllll}44.8 & 45.4 & 45.6 & 45.9 & 32.02\end{array}$

$\begin{array}{llllll}44.5 & 45.0 & 46.2 & 46.6 & 31.58 & -\end{array}$

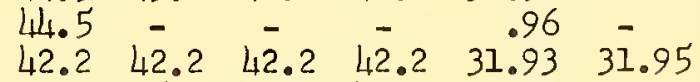
$\begin{array}{llllll}45.4 & 45.5 & 45.5 & 45.7 & 32.08 & \text { - }\end{array}$

$\begin{array}{llllll}44.4 & 44.5 & 44.6 & 45.5 & 31.85 & 31.96\end{array}$ 
Surface water temperature readinss commenced on Georges Shoal on 21 April 1956, well after the winter minimum, and surface and bottom salinities are available for short periods from time to time.

Surface temperatures reached slightly over $60^{\circ} \mathrm{F}$ in mid-September. Values for other depths are not given herein inasmuch as all bathythermomrams indicated an isothermal condition.

Surface salinities ranged between 32.3 and $33.0 \%$. The occasional bottom salinity samples collected yielded salinity values within several hundredths of a $\%$ or the surface salinity, so it is reasonable to assume the water at this location was completely mixed from top to bottom ( $56^{\prime}$ ).

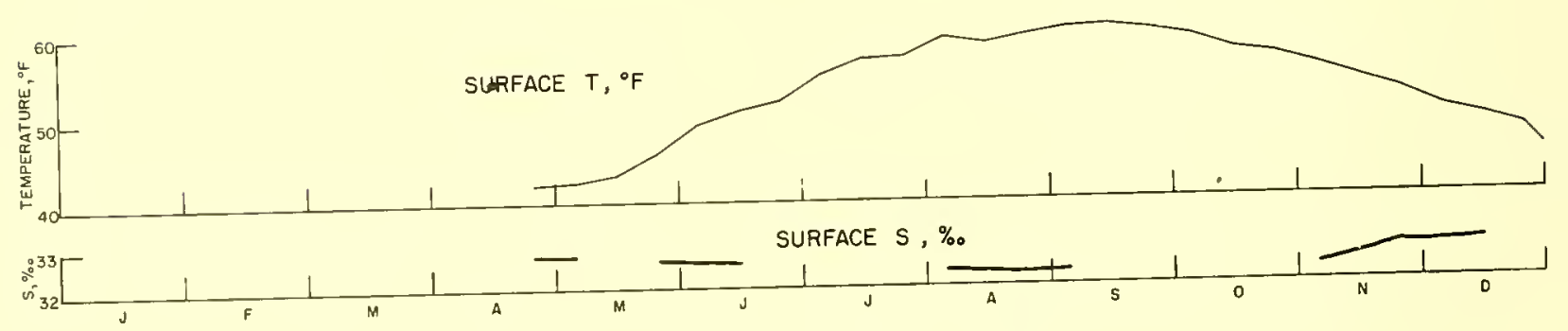

Figure 6. Georres Shoal, 1956. 
TEXAS TOWER \#2

$41^{\circ} 41176.3^{\prime \prime N} .67^{\circ} 45^{\prime} 36.2^{\prime \prime W}$

Depth of water - 56 feet

GEORGES SHOAL

Surface Temperature ${ }^{\circ} \mathrm{F}$

1956

Apri1

May

June

Juzy

August

Month

Da

3

10

\section{1}

\section{2}

13

11)

15

16

17

18

19

20

Temp Sal

Temp Sal

Temp Sal

$42.3 \quad 32.83$

42.5

42.3

42.4

42.2

42.4

42.5

42.4

42.9

43.4

.78

$48.1 \quad 32.60$

48.1

48.7

49.0

49.6

49.0

49.7

49.5

49.7

.77

43.2

42.6

43.6

42.3

42.4

42.7

43.5

43.2

43.8

44.3

32.77

49.2

.66

.65

.58

.56

.62

.56

.56

.56

49.0

-

-

48.9

.70
.56

50.4

51.1

32.56

50.4

它. 6

52.6

52.5

50.4

50.2

-

$-$

$-$

44.4

4.8

44.8

45.4

45.5

45.3

46.7

47.2

50.0

50.1

50.8

51.2

57.0

52.1

$$
52.1
$$

52.5

28 42. $\quad .78$

45.8

46.4

47.7

32.61

.70

53.6

53.6

31

Mean

43.9

50.5

56.1

56.8

56.5

56.2

56.2

56.2

56.4

56.1

55.8

57.0

58.5

59.2

Sal

Temp Sal

$53.4-59.6$

54.5

59.0

58.4

59.5

59.7

59.6

59.0

58.6

58.9

32.37

.37

.38

.37

.36

.34

.32

$53.8=$

$55.4-56.2 \quad .36$

$55.2 \quad-56.4 \quad .37$

56.3

59.7
$-\quad 58.7$

.36

.37

$58.3 \quad .34$

$58.5 \quad .32$

$59.2 \quad .31$

$59.5 \quad .32$

$59.7 \quad .32$

Water Temperatures means of four observations per day, courtesy 12 th Wea. Sqdn., USAF. Water samples collected by U. S. N., Hydro graphic Office, one per day. 
TEXAS TOWER \#2 GEORGES SHOAL $41^{\circ} 41116.3^{\prime \prime N} .67 \% 5^{\prime} 36.2^{\prime \prime}$. Depth of water -56 feet

\section{Surface Temperature OF}

1956

Sept.

$\underline{\text { Oct. }}$

Nov.

Dec.

$\frac{\text { Month }}{\text { Day }}$

\begin{tabular}{lll}
3 & 60.2 & .30 \\
\hline & 60.4 & .29
\end{tabular}

$4 \quad 60.3 \quad .30$

$5 \quad 60.7 \quad 32.38$

$660.1=$

$760.6=$

$860.1=$

$\begin{array}{rrr}9 & 59.1 & -\end{array}$

$\begin{array}{llll}11 & 59.4 & - & 56.1 \\ 12 & 59.8 & - & 56.0\end{array}$

$12 \quad 59.8=$

$\begin{array}{lll}13 & 60.2 & - \\ 15 & 60.7 & -\end{array}$

$1560.2=$

$16 \quad 60.0$ -

1760.7 -

1860.5 -

$19 \quad 59.8$ -

2060.6 -

$21 \quad 60.7$

2260.6 -

$23 \quad 59.7$

$24 \quad 59.9$ -

$\begin{array}{lll}25 & 60.1 \\ 26 & 59.4 & -\end{array}$

$\begin{array}{lll}26 & 59.4 & -\end{array}$

$\begin{array}{lll}28 & 58.4 & -\end{array}$

2959.0 -

$30 \quad 59.6$ -

31
Temp Sal

59.8

59.6

59.4
59.1

58.8

57.8

58.7

58.5

58.8

58.2

57.1

57.6

58.2

58.3

58.2

58.2

57.2

56.4 -

$57.6=$

58.3

58.0

57.4

55.6

55.5

56.2

56.4

56.0

56.4

56.8
$-$

$-$

$-$

$-$

-

-

$-$

$-$

三

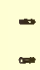

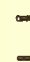

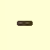

-

$-$

$-$

$-$

$-$

-

$-$

$-$

-

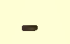

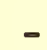

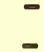

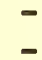

-

-
Temp Sal

$56.8 \quad 32.30$

55.8

55.1

55.2

55.1

54.9

55.5

55.7

55.1

54.0

53.4

54.2

53.6

53.4

54.4

54.8

54.8

54.5

5. 8

52.2

53.5

54.2

53.3

5.8

51.4

52.1

51.8

52.0

51.8
50.9
.28

.30

.47

.31

.35

.37

.44

.46

.54

.52

.66

.53

.49

.61

.66

.64

.57

.76

.84

.73

.73

.79

.78

.78

32.95

33.04

32.94

32.94

$-$
Temp Sal

$51.1 \quad 32.94$

$50.3 \quad .83$

$50.9 \quad .82$

5.5.84

$48.8 \quad .84$

$49.5 \quad .84$

$50.4 \quad .82$

$50.3 \quad .85$

$49.2 \quad .85$

$48.7 \quad .81$

$48.0 \quad .84$

$49.4 \quad .88$

$50.0 \quad 32.91$

$49.3 \quad 33.02$

$49.2 \quad 32.90$

$49.2 \quad 32.84$

49.4 -

$49.6=$

48.0 -

$47.6=$

$47.4=$

$47.9=$

$47.3=$

48.2 -

49.1 -

$47.9=$

47.4

47.2

47.6 -

$46.1=$

Mean $59.9=57.6$ - $53.832 .6 I 48.9$ - 
Nantucket Shoal Lightship

Note: Nantucket Lightship moved at the first of the year from $40^{\circ} 37^{\prime} 00^{\prime \prime N}, 69^{\circ} 18130^{\prime \prime} \mathrm{W}$, a position she had occupied for nearly three years, to $40^{\circ} 33^{\prime} 00^{\prime \prime N}, 69^{\circ} 28^{\prime} 00^{\prime \prime W}$.

Minimum surface temperatures of $38^{\circ} \mathrm{F}$ were recorded in early March and maximum temperatures of $70^{\circ} \mathrm{F}$ were recorded in mid-September.

The whole water column was virtually isothermal durin the first five months of the year. The thermocline commenced development in early June and reached its peak in mid-Seotember. Maximum bottom temperatures occurred during the first third of November.

Surface salinity ranged between $32 \%$ and $34.50 / 00$, the minimum occurring in mid-summer and the maximum in mid-september. Bottom salinities were close to the surface salinities for the first two-thirds of the year but were slightly higher during the autumn. 

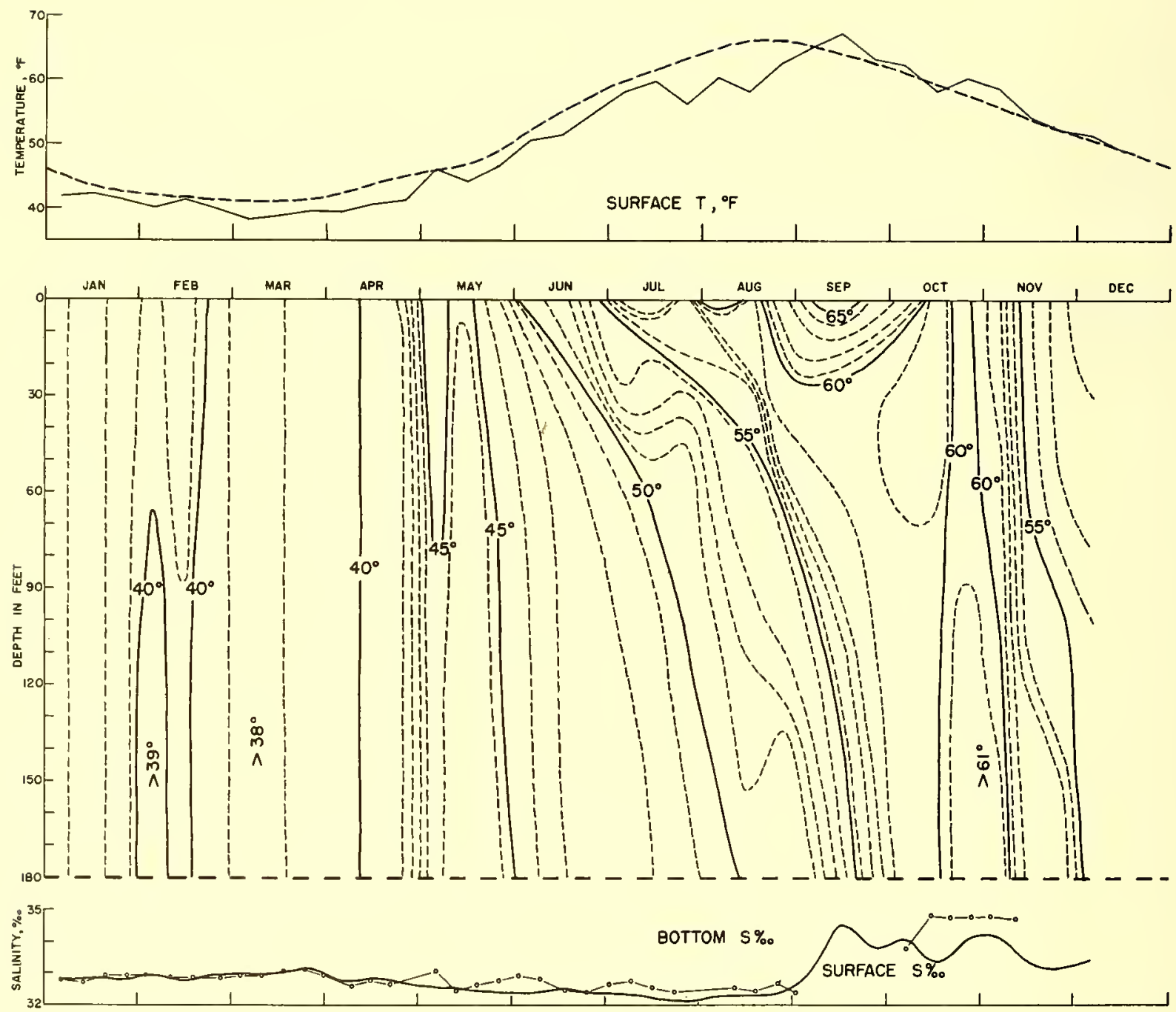

Figure 7. Nantucket Lightship, 1956. (Dashed line in upper diagram, mean for period 1947-1952, 8 miles WSW of present position) 
NANTUCKET SHOALS LICHTSHIP $40^{\circ} 33^{\prime} 00^{\prime \prime} \mathrm{N} .69^{\circ} 28^{\prime} 00^{\prime \prime} \mathrm{W}$.

\section{January 1956}

Temperatare oF

Salinity $\%$

Depth

Day $0^{\prime} \quad 30^{\prime} \quad 50^{\prime} 100^{\prime} 150^{\prime} 180^{\prime}$

1

$4 \quad 41.7 \quad 41.9$

6

7

8

$\begin{array}{ccccccc}9 & - & - & - & - & - & - \\ 10 & 42.3 & 42.3 & 42.3 & 42.4 & 42.4 & 42.4\end{array}$

$\begin{array}{lllllll}11 & 42.0 & 42.1 & 42.1 & 42.1 & 42.0 & 42.0\end{array}$

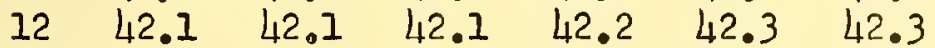

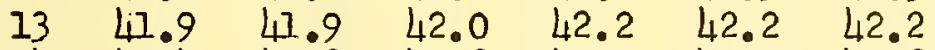

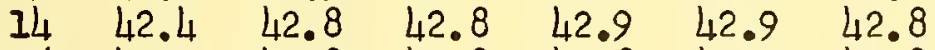

$\begin{array}{lllllll}15 & 42.7 & 42.8 & 42.8 & 42.8 & 42.7 & 42.8\end{array}$

$\begin{array}{lllllll}16 & 42.5 & 42.7 & 42.7 & 42.7 & 42.7 & 42.7\end{array}$

$\begin{array}{lllllll}17 & 42.8 & 42.8 & 42.9 & 42.8 & 42.9 & 42.9\end{array}$

$\begin{array}{lllllll}18 & 42.0 & 42.1 & 42.3 & 42.4 & 42.5 & 42.5\end{array}$

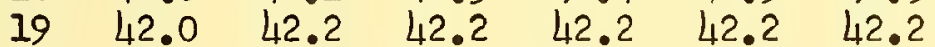

$\begin{array}{lllllll}20 & 42.0 & 42.1 & 42.1 & 42.1 & 42.1 & 42.1\end{array}$

$\begin{array}{lllllll}21 & 42.1 & 42.2 & 42.2 & 42.2 & 42.2 & 42.2\end{array}$

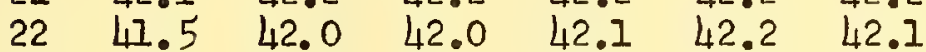

$\begin{array}{lllllll}23 & 41.9 & 41.9 & 41.9 & 42.0 & 47.9 & 42.0\end{array}$

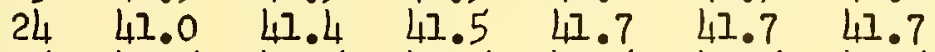

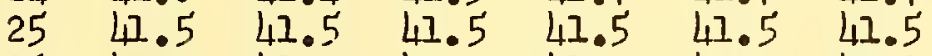

$\begin{array}{lllllll}26 & 41.3 & 47.3 & 47.3 & 47.3 & 47.3 & 47.3\end{array}$

$\begin{array}{lllllll}27 & 40.8 & 40.8 & 40.8 & 47.1 & 41.2 & 41.2\end{array}$

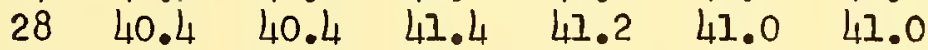

$\begin{array}{lllllll}29 & 40.3 & 40.3 & 40.4 & 41.2 & 41.2 & 41.2\end{array}$

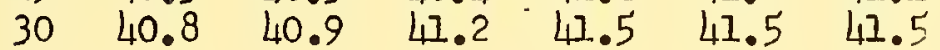

31 - $\quad$ - $\quad$ - $\quad$ - $\quad$ -

$\begin{array}{lllllll}\text { Mean } & 41.7 & 41.8 & 41.9 & 42.0 & 42.0 & 42.0\end{array}$

$32.81 \quad 32.84$ 
NANTUCKET SHOALS LIGHTSHIP $40^{\circ} 33^{\prime} 00^{\prime \prime} \mathrm{N} .69^{\circ} 28^{\prime} 00^{\prime \prime} \mathrm{W}$.

\section{February 1956}

Depth of water-192 feet

Depth

Day $0^{\prime} \quad 30^{\prime} \quad 50^{\prime} 10^{\prime} \quad 150^{\prime} \quad 180^{\prime}$

$\begin{array}{lllllll}1 & 39.8 & 40.2 & 40.1 & 40.1 & 40.2 & 40.0\end{array}$

$\begin{array}{lllllll}2 & 40.4 & 40.4 & 40.3 & 38.9 & 38.8 & 38.8\end{array}$

$\begin{array}{lllllll}3 & 40.7 & 40.6 & 40.7 & 40.7 & 40.7 & 40.7\end{array}$

$\begin{array}{lllllll}4 & 39.4 & 39.7 & 39.7 & 39.7 & 39.5 & 39.6\end{array}$

$\begin{array}{lllllll}5 & 39.4 & 39.4 & 39.5 & 39.7 & 39.7 & 39.7\end{array}$

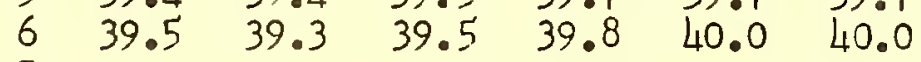

$\begin{array}{lllllll}7 & - & - & - & - & - & - \\ 8 & - & - & - & - & - & -\end{array}$

$\begin{array}{lllllll}9 & 40.2 & 40.1 & 40.1 & 40.0 & 39.9 & 39.9\end{array}$

$\begin{array}{lllllll}10 & 40.3 & 40.2 & 40.2 & 40.1 & 40.1 & 40.1\end{array}$

$\begin{array}{lllllll}11 & 47.8 & 41.7 & 47.7 & 40.8 & 40.4 & 39.3\end{array}$

$\begin{array}{lllllll}12 & 41.5 & 41.5 & 41.4 & 41.3 & 40.7 & 40.6\end{array}$

$\begin{array}{lllllll}13 & 47.6 & 41.6 & 47.4 & 40.9 & 40.8 & 40.8\end{array}$

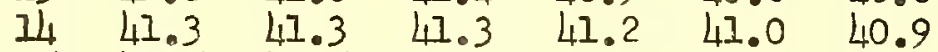

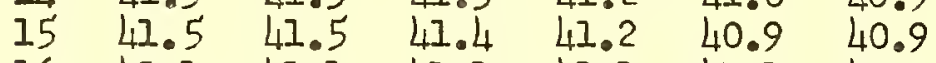

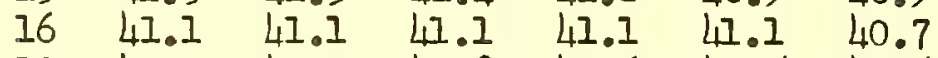

$\begin{array}{lllllll}17 & 40.7 & 40.7 & 40.8 & 40.6 & 40.5 & 40.5\end{array}$

$\begin{array}{lllllll}28 & 40.7 & 40.8 & 40.8 & 40.8 & 40.7 & 40.7\end{array}$

$\begin{array}{lllllll}19 & 40.5 & 40.9 & 40.8 & 40.4 & 40.2 & 40.2\end{array}$

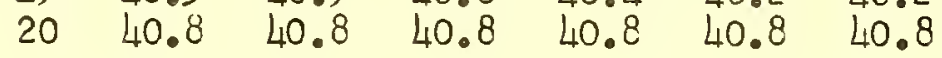

Salinity $\%$

$\begin{array}{lllllll}21 & 40.8 & 40.7 & 40.7 & 40.7 & 40.7 & 40.7\end{array}$

$\begin{array}{lllllll}22 & 40.3 & 40.3 & 40.4 & 40.4 & 40.4 & 40.4\end{array}$

$\begin{array}{lllllll}23 & 39.5 & 39.7 & 39.7 & 39.6 & 39.6 & 39.6\end{array}$

$\begin{array}{lllllll}24 & 39.0 & 39.3 & 39.4 & 39.4 & 39.4 & 39.4\end{array}$

$25-0 \quad-\quad-\quad-\quad-\quad-$

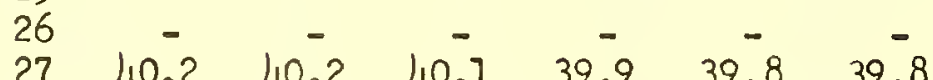

$\begin{array}{lllllllll}28 & 40.2 & 40.2 & 40.1 & 39.9 & 39.8 & 39.8\end{array}$

$\begin{array}{lllllll}29 & 38.8 & 39.0 & 39.0 & 39.0 & 38.9 & 38.9\end{array}$

30

31

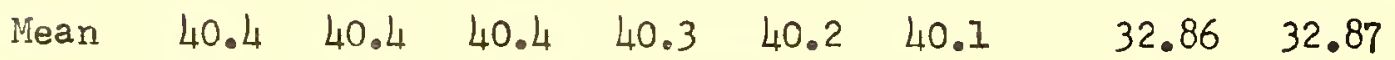


NANTUCKET SHOALS LIGHTSHIP $40^{\circ} 33^{\prime} 00^{\prime \prime N}$. $69^{\circ} 28^{\prime} 00^{\prime \prime}$. Depth of water - 192 feet March 1956

$$
\text { Temperature }{ }^{\circ} \mathrm{F}
$$

Depth $\begin{array}{lllllll}1 & 38.6 & 38.5 & 38.5 & 38.5 & 38.5 & 38.5\end{array}$

$\begin{array}{lllllll}2 & 38.3 & 38.3 & 38.3 & 38.3 & 38.2 & 38.2\end{array}$

$\begin{array}{lllllll}3 & 38.3 & 38.3 & 38.3 & 38.3 & 38.5 & 38.3\end{array}$

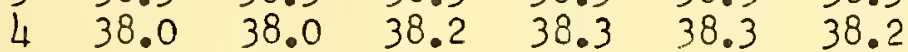

$\begin{array}{lllllll}5 & 38.0 & 37.7 & 38.0 & 38.0 & 38.0 & 38.0\end{array}$

$\begin{array}{lllllll}6 & 38.0 & 37.7 & 38.0 & 38.2 & 38.2 & 38.2\end{array}$

$\begin{array}{lllllll}7 & 38.7 & 38.4 & 38.4 & 38.5 & 38.3 & 38.4\end{array}$

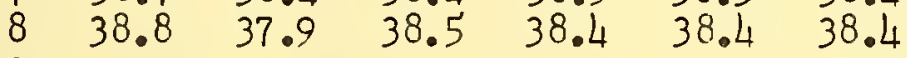

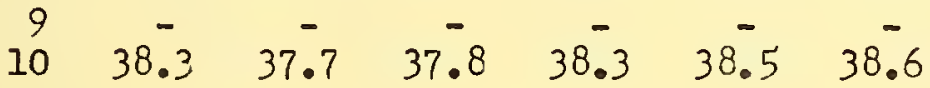

$\begin{array}{lllllll}17 & 38.0^{\circ} & 38.2 & 38.1 & 38.0 & 38.0 & 38.0\end{array}$

$\begin{array}{lllllll}12 & 38.3 & 38.3 & 38.3 & 38.3 & 38.4 & 38.4\end{array}$

$\begin{array}{lllllll}13 & 38.4 & 38.4 & 38.5 & 38.5 & 38.5 & 38.5\end{array}$

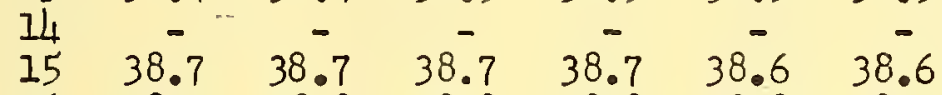

$\begin{array}{lllllll}16 & 38.7 & 38.8 & 38.8 & 38.8 & 38.8 & 38.9\end{array}$

$\begin{array}{lllllll}17 & 39.7 & 39.7 & 39.7 & 39.6 & 39.6 & 39.6\end{array}$

$\begin{array}{lllllll}18 & 39.6 & 39.6 & 39.6 & 39.5 & 39.6 & 39.4\end{array}$

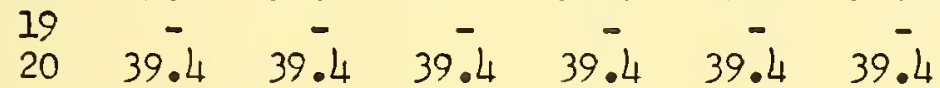

$\begin{array}{lllllll}21 & 39.6 & 39.7 & 39.7 & 39.6 & 39.5 & 39.5\end{array}$

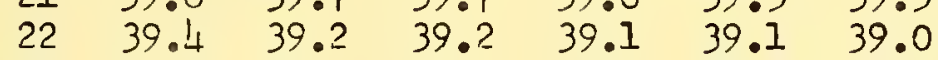

$\begin{array}{lllllll}23 & 39.7 & 39.7 & 39.7 & 39.7 & 39.7 & 39.6\end{array}$

$\begin{array}{lllllll}24 & 39.7 & 39.7 & 39.7 & 39.6 & 39.5 & 39.5\end{array}$

$\begin{array}{lllllll}25 & 39.2 & 39.8 & 39.8 & 39.8 & 39.6 & 39.5\end{array}$

$\begin{array}{lllllll}26 & 39.7 & 39.7 & 39.7 & 39.7 & 39.7 & 39.7\end{array}$

$\begin{array}{lllllll}27 & 39.8 & 39.8 & 39.8 & 39.8 & 39.7 & 39.8\end{array}$

$\begin{array}{lllllll}28 & 39.5 & 39.5 & 39.5 & 39.5 & 39.5 & 39.5\end{array}$

$\begin{array}{lllllll}29 & 39.5 & 39.3 & 39.3 & 39.2 & 39.2 & 39.2\end{array}$

$\begin{array}{lllllll}30 & 39.3 & 39.5 & 39.4 & 39.3 & 39.2 & 39.2\end{array}$

$\begin{array}{lllllll}31 & 39.0 & 39.3 & 39.2 & 39.2 & 39.1 & 39.1\end{array}$

$\begin{array}{lllllll}\text { Hean } & 38.9 & 38.9 & 38.9 & 38.9 & 38.9 & 38.9\end{array}$
Salinity $\%$

$0^{\prime} \quad 180^{\prime}$

$\begin{array}{rc}32.97 & - \\ .99 & - \\ .89 & 32.93 \\ .98 & - \\ 32.87 & - \\ 33.04 & - \\ 32.89 & - \\ .90 & - \\ - & -\end{array}$

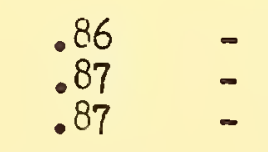

$32.88=$

33.02

$.11 \quad 33.09$

$.14 \quad-$

$.07 \quad-$

$\begin{array}{cc}.15 & - \\ .16 & - \\ .16 & - \\ .05 & 33.11 \\ .16 & - \\ .13 & - \\ .10 & - \\ .14 & - \\ .09 & - \\ .33 & - \\ .23 & 32.95\end{array}$

$33.03 \quad 33.00$ 
Table 6 (cont'd)

NANTUCKET SHOALS LIGHTSHIP 40033'00"N. 69028100"W. Depth of water - 192 feet April 1956

$$
\text { Temperature }{ }^{\circ} \mathrm{F}
$$

Depth

Day

$\frac{10}{2}$

3

4

5

$\begin{array}{lllllll}8 & 39.0 & 30.0 & 39.0 & 39.1 & 39.1 & 39.1\end{array}$

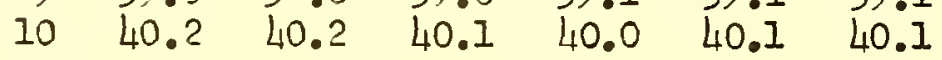

$\begin{array}{lllllll}11 & 40.1 & 39.9 & 39.9 & 39.9 & 39.9 & 39.9\end{array}$

$\begin{array}{lllllll}I 2 & 40.0 & 40.0 & 40.1 & 40.1 & 40.0 & 40.0\end{array}$

$\begin{array}{lllllll}13 & 40.9 & 40.6 & 40.5 & 40.4 & 40.4 & 40.4\end{array}$

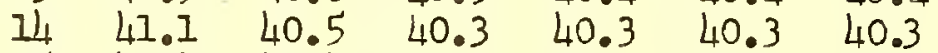

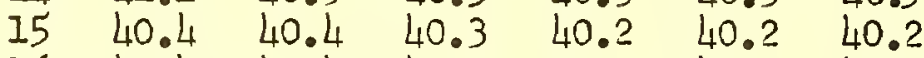

$\begin{array}{lllllll}16 & 40.4 & 40.4 & 40.3 & 40.3 & 40.3 & 40.3\end{array}$

$\begin{array}{lllllll}17 & 39.9 & 39.7 & 39.5 & 39.5 & 39.5 & 39.8\end{array}$

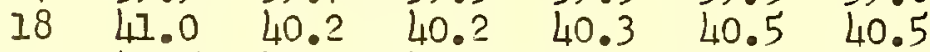

$\begin{array}{lllllll}19 & 41.5 & 40.5 & 40.4 & 40.4 & 40.4 & 40.4\end{array}$

$\begin{array}{lllllll}20 & 40.8 & 40.6 & 40.5 & 40.5 & 40.5 & 40.5\end{array}$

$\begin{array}{lllllll}21 & 40.5 & 40.5 & 40.5 & 40.5 & 40.7 & 40.6\end{array}$

$\begin{array}{lllllll}22 & 40.7 & 40.7 & 40.7 & 40.5 & 40.5 & 40.5\end{array}$

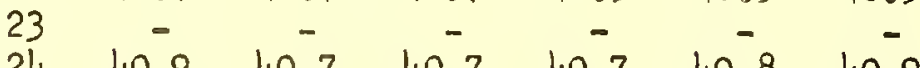

$\begin{array}{lllllll}24 & 40.9 & 40.7 & 40.7 & 40.7 & 40.8 & 40.9\end{array}$

$\begin{array}{lllllll}25 & 41.5 & 47.1 & 47.0 & 40.9 & 41.0 & 41.0\end{array}$

$\begin{array}{lllllll}26 & 40.9 & 47.0 & 41.0 & 41.0 & 47.0 & 41.0\end{array}$

$\begin{array}{lllllll}27 & 41.3 & 41.1 & 40.9 & 41.0 & 41.0 & 41.0\end{array}$

$\begin{array}{lllllll}28 & 41.3 & 40.9 & 40.9 & 41.1 & 41.0 & 41.1\end{array}$

$\begin{array}{lllllll}29 & 47.8 & 47.5 & 41.5 & 41.5 & 41.3 & 47.4\end{array}$

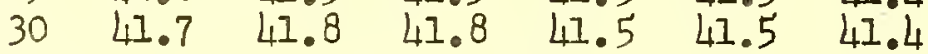

31
Salinity $\%$

$0^{\prime} \quad 180^{\prime}$

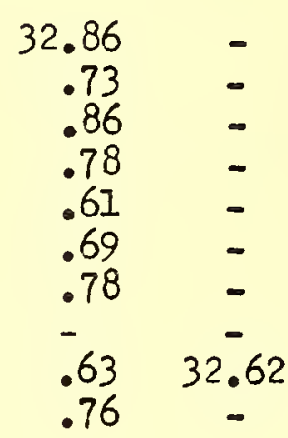

$\begin{array}{rc}.74 & - \\ .88 & - \\ 32.81 & - \\ 33.02 & - \\ 32.79 & 32.77 \\ .85 & - \\ .71 & - \\ .88 & - \\ .66 & - \\ .84 & -\end{array}$

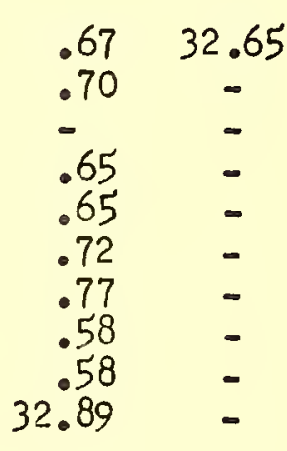

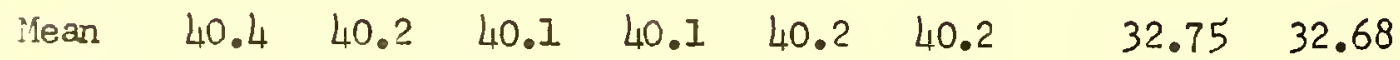




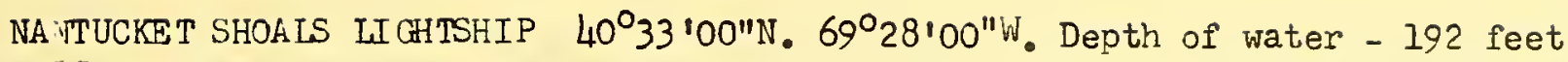
May 1956

Depth

Day $0^{\prime} \quad 30^{\prime} 50^{\prime} 100^{\prime} 150^{\prime} 180^{\prime} \quad 0^{\prime} 180^{\prime}$

$1 \quad 46.0 \quad 45.2 \quad 45.0 \quad 45.0 \quad 45.0 \quad 45.0$

$\begin{array}{lllllll}2 & 46.6 & 45.2 & 45.1 & 45.0 & 45.1 & 45.1\end{array}$

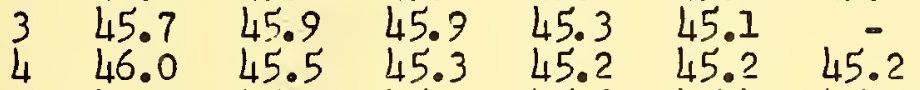

$\begin{array}{lllllll}5 & 47.0 & 45.7 & 45.5 & 45.8 & 45.4 & 45.3\end{array}$

$\begin{array}{lllllll}6 & 47.0 & 46.0 & 45.8 & 45.6 & 45.3 & 45.2\end{array}$

$\begin{array}{lllllll}7 & 46.4 & 46.1 & 46.1 & 45.1 & 45.1 & 45.0\end{array}$

$\begin{array}{lllllll}8 & - & - & - & - & - & -\end{array}$

10

$\begin{array}{llllll}11 & 43.9 & 43.2 & 43.0 & 42.8 & 42.8\end{array}$

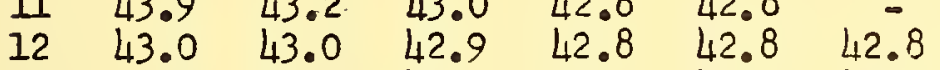

$\begin{array}{lllllll}13 & 43.5 & 43.1 & 43.0 & 43.0 & 43.0 & 43.0\end{array}$

$\begin{array}{lllllll}14 & 44.0 & 43.5 & 43.2 & 43.1 & 43.1 & 43.1\end{array}$

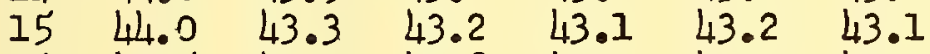

$\begin{array}{lllllll}16 & 43.5 & 42.7 & 42.8 & 43.0 & 43.0 & 43.0\end{array}$

17 44. - $\quad-\quad$ - $\quad$ -

18 44. -

19 46. - $\quad-\quad-\quad-$

20 45. - - - - - -

2146.5 - $\quad$ - $\quad$ - $\quad$ -

$22 \quad 47.5 \quad-\quad-\quad-5.5$

$23 \quad 47.5 \quad-\quad-\quad-\quad-\quad-$

$\begin{array}{lllll}24 & 45.5 & - & - & -\end{array}$

$26 \quad 47.5=-\quad-\quad-$

$2746.5=-\quad-$

$28 \quad 45$.

2945.

$30 \quad 46.5$

$31 \quad 46.5$

Mean 45.5 - $\quad$ - $\quad$ - $\quad$ - $\quad 32.5132 .74$

Mean 45.5 - $\quad$ - $\quad$ - $\quad$ - 32.5132 .74

$-\quad-\quad-\quad-$

$-$

$-$

$\begin{array}{ll}.55 & - \\ .48 & 32.47\end{array}$

$.51-$

.080

$.47=$

$.53-$

$.53 \quad 32.65$

$\begin{array}{rrc}.54 & - \\ - & .57 & - \\ & .49 & - \\ - & .52 & - \\ - & .59 & - \\ - & .42 & 32.77 \\ - & .38 & - \\ - & .40 & - \\ - & .36 & - \\ - & .46 & - \\ - & 32.38 & -\end{array}$ 
NATTUCKET SHOALS LIGHTSHIP $40^{\circ} 33^{1} 00^{\prime \prime N}$. 69028100"W. Depth of water - 192 feet June 1956

Temperature ${ }^{\circ} \mathrm{F}$

Depth $0^{\prime} \quad 30^{\prime}$ 50

$\begin{array}{ll}1 & 46.5 \\ 2 & 48 . \\ 3 & 48 . \\ 4 & 50 . \\ 5 & 51.5 \\ 6 & 52 . \\ 7 & 53 . \\ 8 & 55 . \\ 9 & 50 . \\ 10 & 52 .\end{array}$

17

12

1350.

1450 .

1551.

16

17

18

19

20

$21 \quad 54 . \quad 48.9 \quad 47.2 \quad 47.2 \quad 47.3 \quad 47.3$ $\begin{array}{lllllll}22 & 55.9 & 42.9 & 49.6 & 46.2 & 46.2 & 46.2\end{array}$

$\begin{array}{lllllll}23 & 56.6 & 53.3 & 49.7 & 47.2 & 47.3 & 47.2\end{array}$

$\begin{array}{lllllll}24 & 53.8 & 48.9 & 47.9 & 47.8 & 47.7 & 47.6\end{array}$

$\begin{array}{lllllll}25 & 54.0 & 48.7 & 48.3 & 47.8 & 47.7 & 47.7\end{array}$

$\begin{array}{lllllll}26 & 51.2 & 48.9 & 48.5 & 48.2 & 47.9 & 47.8\end{array}$

$\begin{array}{lllllll}27 & 51.0 & 49.4 & 49.0 & 48.4 & 48.1 & 48.0\end{array}$

$\begin{array}{lllllll}28 & 55.9 & 50.9 & 49.4 & 48.7 & 48.4 & 48.0\end{array}$

$\begin{array}{lllllll}29 & 57.8 & 50.7 & 48.9 & 48.1 & 48.0 & 48.0\end{array}$

$\begin{array}{lllllll}30 & 57.3 & 51.0 & 49.1 & 48.5 & 47.9 & 48.1\end{array}$
Salinity $\%$

$0^{\prime} \quad 180^{\prime}$

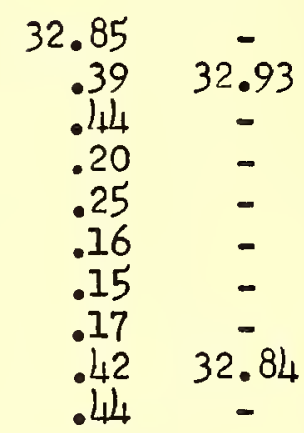

$.38=$
$.75=$
.48
.69
.39

$.30 \quad 32.47$

$.53 \quad-$

$.61 \quad-$

$.54 \quad$.

$\begin{array}{cc}.56 & = \\ .54 & = \\ .47 & - \\ .19 & 32.47 \\ .33 & = \\ .26 & = \\ .26 & - \\ .30 & - \\ .33 & = \\ 2.33 & =\end{array}$

Mean $52.3 \quad$ - $\quad$ - $\quad$ - $\quad$ - $\quad$ - $\quad 32.4732 .66$ 
NANTUCKE T SHOALS LIGHTSHIP $40^{\circ} 33^{\prime} 00^{\prime \prime} \mathrm{N}_{0} 69^{\circ} 28^{\prime} 00^{\prime \prime} \mathrm{W}$. Depth of water - 192 feet

\section{Juzy 1956}

Temperatare ${ }^{\circ} \mathrm{F}$

Depth

Day

$\frac{1}{2}$

11

12

1

14

15

16

17

18

20

21

22

23

24

25

26

27

28

29

30

31

11 $62.0 \quad 52.7$

54.05

50.7

49.0 58.5 50.6 49.8 48.5 60.0 51.2 53.9 50.3 49.1 60.6 54.0 64.1 61.0 51.1 48.3

1

57.8 54.0 52.7 55.0 62.7 53.5 58.8 58.8

55.0

51.0 $50.9 \quad 50.6$ 51.950 .2 51.0 50.5 51.0 51.151 .0 51.450 .9 $55.7 \quad 51.3$ 52.0 53.3 52.3 $0^{\prime} 30^{\prime} 50^{\prime} 100^{\prime} 150^{\prime} \quad 180^{\prime}$

$\begin{array}{llllll}55.7 & 49.0 & 48.7 & 48.4 & 47.4 & 47.0\end{array}$ $\begin{array}{llllll}59.5 & 51.3 & 48.9 & 47.6 & 47.1 & 47.0\end{array}$ $\begin{array}{llllll}61.0 & 56.8 & 48.9 & 48.6 & 47.1 & 47.1\end{array}$

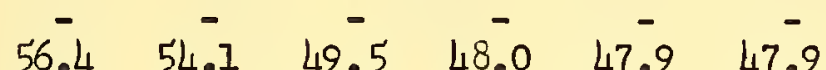

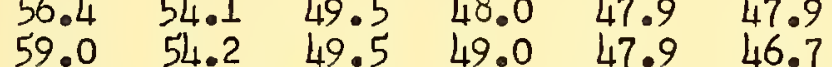
$\begin{array}{llllll}57.0 & 56.0 & 50.0 & 48.0 & 47.5 & 47.3\end{array}$ $\begin{array}{llllll}57.0 & 50.0 & 48.7 & 48.8 & 48.6 & 48.5\end{array}$ $\begin{array}{llllll}58.8 & 58.2 & 51.0 & 48.1 & 48.1 & 48.1\end{array}$

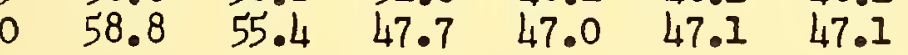

$4 \overline{7} . \overline{7} \quad 4 \overline{7.5}$ $48.2 \quad 48.2$ 48.348 .3 $50.8 \quad 49.0 \quad 48.1 \quad 48.1$ $47.5 \quad 47.3$ $55.0 \quad 48.4 \quad 47.5 \quad 47.5$

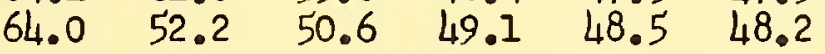
$\begin{array}{cccccc}53.8 & 50.8 & 50.2 & 49.2 & 49.0 & 48.9\end{array}$ 51.8
51.3

51.1
$49.1 \quad 49.1 \quad 48.6$ $49.9 \quad 49.1 \quad 49.0$ $\begin{array}{lll}49.8 & 49.2 \quad 49.2\end{array}$ $\begin{array}{lll}50.0 & 49.1 & 48.3\end{array}$ $50.4 \quad 50.0 \quad 50.0$ $50.0 \quad 49.2 \quad 49.0$ $50.5 \quad 49.1 \quad 48.9$ $50.4 \quad 48.0 \quad 47.7$ $50.6 \quad 49.0 \quad 47.8$ $\begin{array}{lll}50.7 & 49.8 \quad 48.7\end{array}$
Salinity $\%$

$0^{\prime} \quad 180^{\prime}$

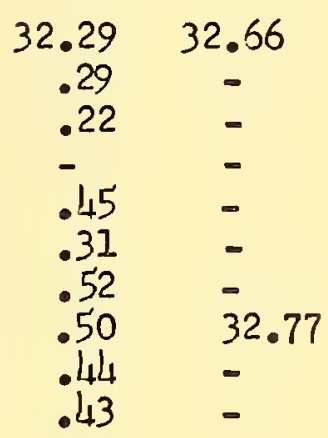

.42

$\begin{array}{cc}.422 & - \\ .30 & - \\ . & - \\ .29 & 32.54 \\ .26 & - \\ .08 & = \\ .16 & - \\ . & - \\ .23 & -\end{array}$

$\begin{array}{rc}.748 & 32.44 \\ .20 & = \\ .14 & = \\ 32.16 & = \\ 31.19 & - \\ 32.21 & - \\ .18 & - \\ .20 & - \\ .22 & - \\ 32.28 & -\end{array}$ 
NA TUCKET SHOALS IIGH TSHIP $40^{\circ} 33^{\prime} 00^{\prime \prime N}$. 69028 $8^{\prime} 00^{\prime \prime}$ W. Depth of water - 192 feet August 1956

Temperature ${ }^{\circ} \mathrm{F}$

Salini ty $\%$

Depth

Day $0^{\prime} \quad 30^{\prime} 50^{\prime} 100^{\prime} 150^{\prime} 180^{\prime}$

$0^{\prime} \quad 180^{\prime}$

$\begin{array}{lllllll}1 & 62 . & 53.4 & 42.0 & 51.5 & 50.8 & 50.5\end{array}$

$\begin{array}{lllllll}2 & 57.0 & 52.3 & 51.4 & 50.5 & 49.7 & 49.0\end{array}$

$\begin{array}{lllllll}3 & 54.3 & 53.8 & 52.9 & 52.2 & 49.8 & 48.1\end{array}$

$\begin{array}{lllllll}4 & 63.6 & 61.5 & 58.0 & 49.3 & 48.8 & 48.6\end{array}$

$\begin{array}{lllllll}5 & 64.3 & 56.3 & 52.6 & 50.1 & 49.2 & 49.2\end{array}$

$\begin{array}{lllllll}6 & 63.5 & 55.7 & 53.0 & 50.7 & 49.3 & 49.3\end{array}$

$7 \quad-\quad-\quad-\quad-\quad-$

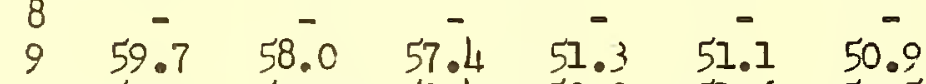

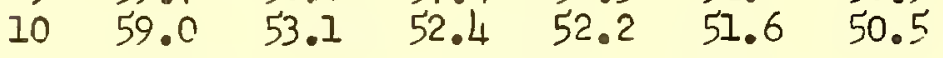

$\begin{array}{lllllll}11 & 55.7 & 53.3 & 52.3 & 52.1 & 51.3 & 50.6\end{array}$

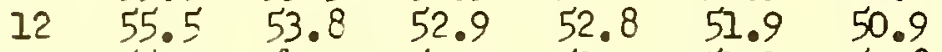

$\begin{array}{lllllll}13 & 55.3 & 54.0 & 52.9 & 52.0 & 51.1 & 50.8\end{array}$

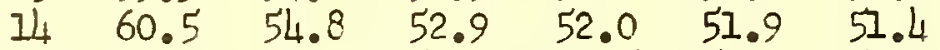

$\begin{array}{lllllll}15 & 59.0 & 54.2 & 53.0 & 52.5 & 52.1 & 52.0\end{array}$

$\begin{array}{lllllll}16 & 55.3 & 52.8 & 53.0 & 52.8 & 52.7 & 51.0\end{array}$

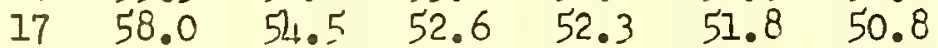

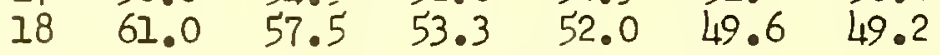

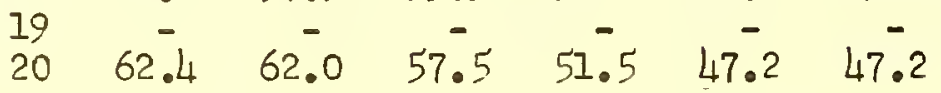

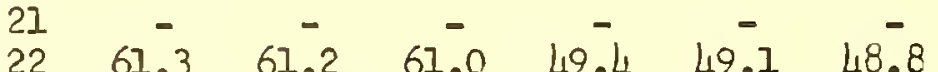

$\begin{array}{lllllll}22 & 61.3 & 61.2 & 61.0 & 49.4 & 49.1 & 48.8 \\ 23 & 61.3 & 61.1 & 61.0 & 54.0 & 50.5 & 50.1\end{array}$

$\begin{array}{lllllll}24 & 62.4 & 60.9 & 60.5 & 53.1 & 50.8 & 50.4\end{array}$

$\begin{array}{lllllll}25 & 59.0 & 57.9 & 56.8 & 53.0 & 49.8 & 49.8\end{array}$

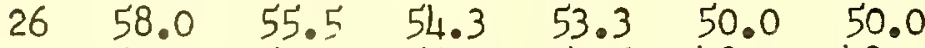

$\begin{array}{lllllll}27 & 62.7 & 57.9 & 56.0 & 52.6 & .48 .7 & 48.3\end{array}$

$\begin{array}{lllllll}28 & 63.5 & 59.2 & 58.6 & 53.5 & 50.3 & 49.0\end{array}$

$\begin{array}{lllllll}29 & 64.9 & 63.7 & 59.4 & 53.0 & 49.9 & -48.9\end{array}$

$\begin{array}{lllllll}30 & 65.4 & 62.8 & 59.9 & 56.9 & 50.0 & 49.3\end{array}$

$\begin{array}{lllllll}31 & 65.0 & 58.7 & 56.0 & 53.7 & 51.0 & 50.5\end{array}$

$\begin{array}{lllllllll}\text { Mean } & 60.4 & 57.0 & 55.3 & 52.2 & 50.4 & 49.8 & 32.30 & 32.57\end{array}$ 
NANTUCKET SHOALS LIGHTSHIP $40^{\circ} 33^{\prime} 00^{\prime \prime N}$. $69^{\circ} 28^{\prime} \mathrm{C} 0^{\prime \prime} \mathrm{W}$. Depth of water - 192 feet September 1956

Temperature of

Depth $\begin{array}{lllllll}1 & 65.5 & 56.8 & 55.7 & 54.1 & 52.0 & 51.1\end{array}$

$\begin{array}{lllllll}2 & 59.5 & 54.9 & 54.2 & 53.7 & 52.8 & 52.4\end{array}$

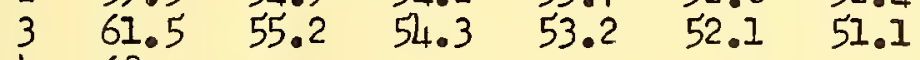

5

468

566

765

867.5

966.

1065.5

1163.

1264.

1365.

I4 69 .

1570 .

1670.

1769.

1870.

1966 .

2067.

2163.

22 64.

23 61.

2459.

2560.

2667.

2765.

2866.

2963.

$30 \quad 66$.

31

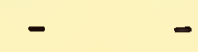

$-$

-

-

-

- $\quad-$

-

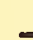

$-$

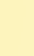

$-$

-

-

-

$-$

$-$

$-$

-

-

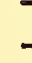

-

$-$

$-$

-

$-$

-

$-$

$-$

-

$-$
Salinity $\%$

$0^{\prime} \quad 180^{\prime}$

$32.41 \quad 32.40$

$.25 \quad-$

$32.18=$

$33.34=$

33.05 -

32.55

32.32

$33.89=$

38.84

$33.85=$

$33.61=$

$33.76=$

$35.10=$

35.07 -

35.43

$34.72=$

35.18 -

34.01 -

34.22 -

$33.76=$

$33.65=$

$32.89=$

$32.89=$

32.77

34.62

34.23

34.83

34.15

34.51

Mean

65.2

- $\quad-$

$-$

33.77 
NANTUCKET SHOALS LIGHTSHIP $40^{\circ} 33^{\prime} 0^{\prime} 0^{\prime N}$. $69^{\circ} 28^{\prime} 00^{\prime \prime} \mathrm{W}$. Depth of water - 192 feet

\section{October 1956}

Temperature ${ }^{\circ} \mathrm{F}$

Depth $0^{\prime} 30^{\prime} 50^{\prime} 100^{\prime} 150^{\prime} 180^{\prime}$

166

265 .

364.

464 .

564

$659.5 \quad 59.5$

7
8

57.7

59.0

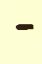

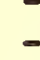

9
10

17

12

13

14

15

16

17

18

19

20

27

22

2

24

25

26

27

28

29

30

31

$57.1 \quad 57.0$

57

58.8

58.9

55.2

58.4

58.4

$57.6 \quad 57.7$

62.0

59.3

60.4

60.9
59.960 .1

$57.2 \quad 57.1 \quad 57.4$

$59.1 \quad 59.1 \quad 59.2$

58.7

58.6

58.9

59.2

59.1

55.3

58.1

55.3

58.4

$62.7 \quad 62.7$

62.0

$58.1 \quad 58.6$

62.962 .9

60.1

59.9

60.9

61.3

62.7

57.962 .1

61.9

$62.9 \quad 62.9$

$58.0 \quad 58.8 \quad 58.7$

59.5

59.2

59.3

59.5

$59.1 \quad 59.2$

$60.1 \quad 60.1 \quad 60.0$

$59.7 \quad 59.3$

59.2

$55.9 \quad 56.0 \quad 56.1$

$58.7 \quad 58.8 \quad 58.7$

$\begin{array}{llll}52.1 & 60.1 & 59.6 & 59.6 \\ & 62.1 & 62.1 & 62.1\end{array}$

$58.6 \quad 58.9 \quad 58.9$

62.9

63.1

63.4

58.8

63.7

$\begin{array}{lll}60.2 & 62.1 & 62.9\end{array}$

63.0

$60.1 \quad 62.1 \quad 62.9$

63.1

61.2

61.8

62.0

62.0

$61.7 \quad 61.9$

61.9

62.0

Mean
59. $\begin{array}{llll}59.4 & 60.2 & 60.5 & 60.5\end{array}$
Salinity $\%$

$0^{\prime} 180^{\prime}$

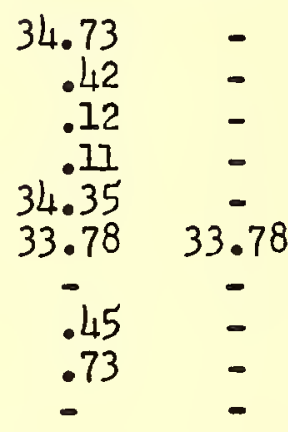

$.16=$

$.37 \quad-$

. $37 \quad 34.83$

.33 -

$.70=$

$.76 \quad-$

$\begin{array}{ll}.35 & - \\ .16 & -\end{array}$

$\begin{array}{ll}.13 & 34.77\end{array}$

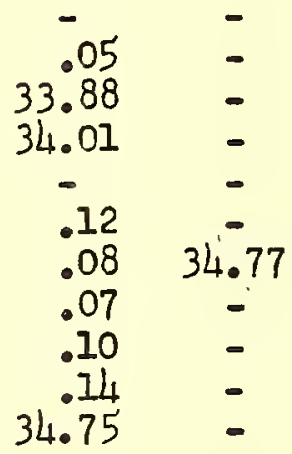

$33.82 \quad 34.54$ 
Table 6 (cont'd)

NANTUCKET SHOALS LIGHTSHIP $40^{\circ} 33^{\prime} 00^{\prime \prime N} .69^{\circ} 28^{\prime} 00^{\prime \prime} \mathrm{W}$. Depth of water - 192 feet November 1956

$$
\text { Temperature OF }
$$

Salinity $\%$

Depth

$\underline{\text { Day }} 0^{\prime} \quad 30^{\prime} \quad 50^{\prime} 100^{\prime} 150^{\prime} 180^{\prime} \quad 0^{\prime} 180^{\prime}$

$\begin{array}{lllllll}1 & 57.9 & 58.2 & 59.9 & 61.7 & 61.9 & 61.8\end{array}$

$34.07=$

$\begin{array}{lllllll}2 & 58.0 & 58.9 & 60.3 & 61.3 & 61.5 & 61.6\end{array}$

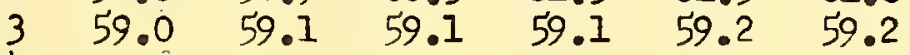

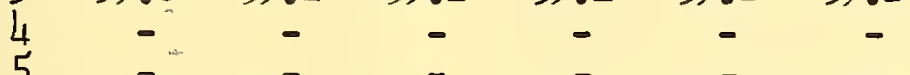

$\begin{array}{lllllll}6 & 59 . & - & - & - & - & -\end{array}$

$\begin{array}{lllll}7 & 61.9 & 61.9 & 61.9 & 61.9\end{array}$

61.961 .9

$\begin{array}{llllll}59.2 & 59.1 & 59.4 & 61.9 & 63.9 & 63.9\end{array}$

$\begin{array}{lllllll}9 & 55.1 & 55.2 & 55.5 & 56.7 & 63.1 & 63.2\end{array}$

$\begin{array}{lllllll}11 & 55.0 & 55.0 & 55.1 & 56.8 & 63.0 & 63.1\end{array}$

12 - $\quad$ - $\quad$ - $\quad$ - $\quad$ -

$\begin{array}{lllllll}13 & - & - & - & - & - & -\end{array}$

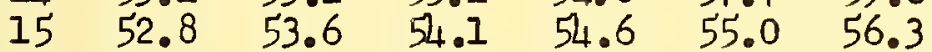

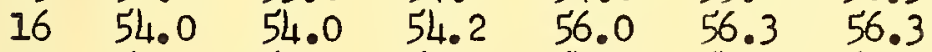

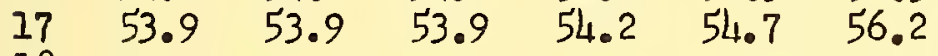

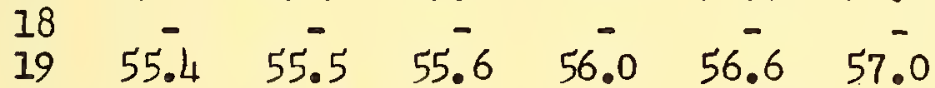

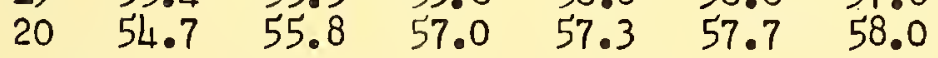

$\begin{array}{ll}33.81 & - \\ 34.32 & 34.81\end{array}$

$\begin{array}{lllllll}21 & 55.9 & 56.6 & 56.9 & 57.6 & 57.7 & 57.9\end{array}$

22 - $\quad$ - $\quad$ - $\quad$ - $\quad$ -

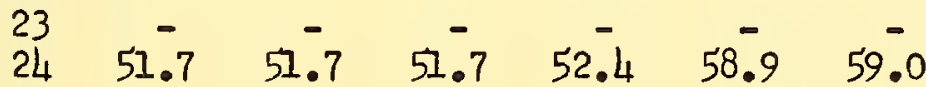

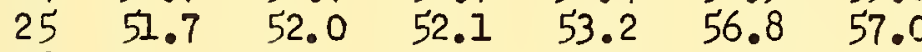

$\begin{array}{lllllll}26 & 5 & - & - & - & - & -\end{array}$

28 - - - - -

$\begin{array}{lllllll}29 & 49.4 & 50.0 & 50.8 & 57.3 & 58.2 & 58.7\end{array}$

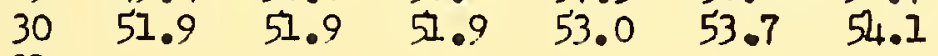

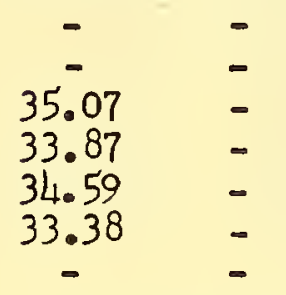

31

Mean

$\begin{array}{llllll}55.1 & 55.4 & 55.9 & 57.4 & 59.1 & 59.4\end{array}$

$.36 \quad 34.71$

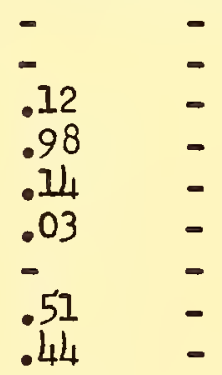

$33.48=$

$-$

$32.98-$

$32.96-$

$33.23=$

32.82 -

33.47

$33.58=$ 
NANTUCKET SHOALS LIGHTSHIP $40^{\circ} 33^{\prime} 00^{\prime \prime N}$. $69^{\circ} 28^{\prime} 00^{\prime \prime}$ W. Depth of water - 192 feet

\section{December 1956}

Temperature ${ }^{\circ} \mathrm{F}$

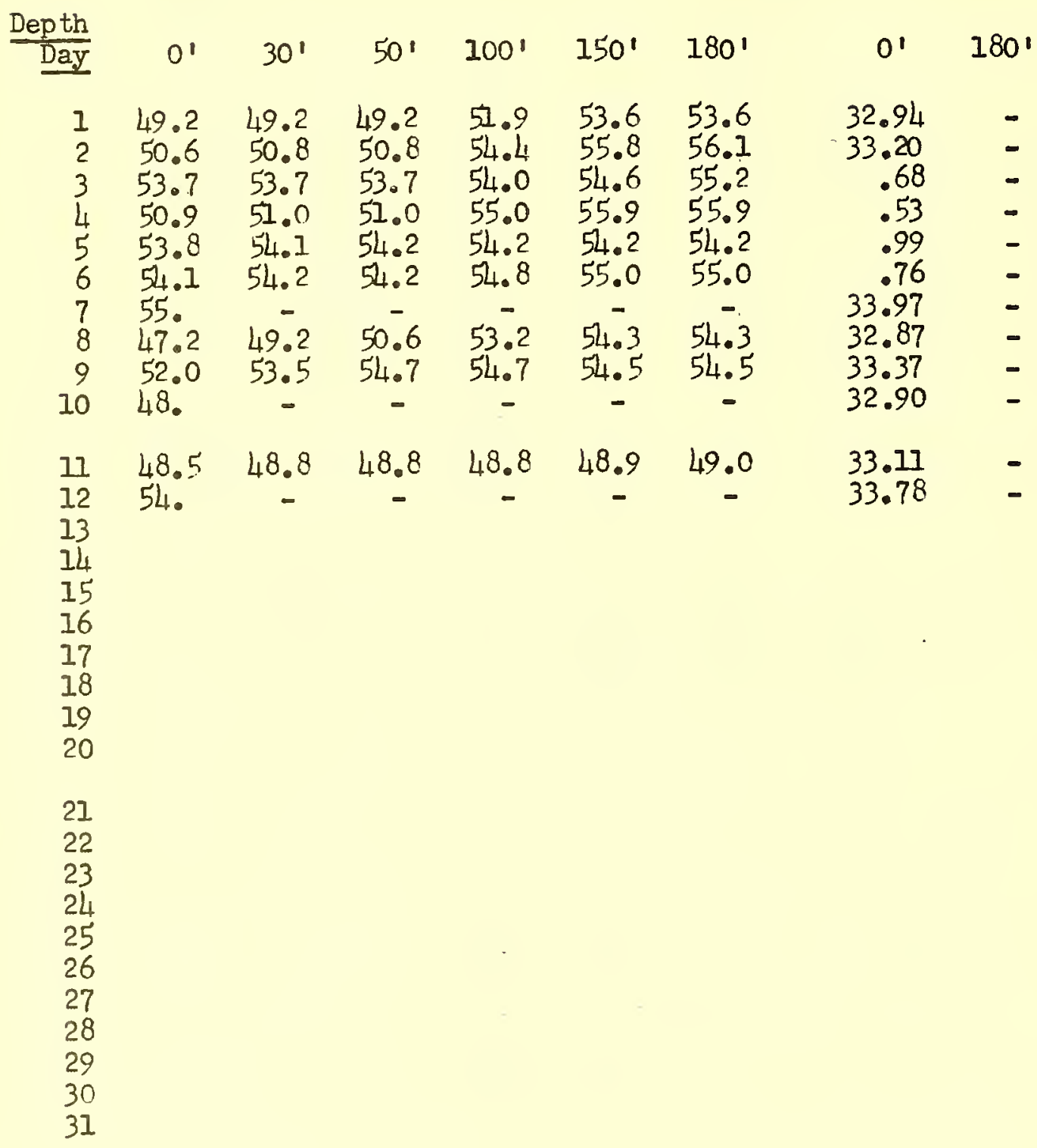




\section{Woods Hole Massachusetts}

Water temperatures at Woods Hole were, on an annual basis, substantially lower than had been observed since 1941, but were comparable to the mean for the period of record. (See Bumpus, 1957) A relatively cold January was followed by a slightly retarded spring. Summer temperatures were comparable to the mean for the period of record, but the autum temperatures cooled more slowly than the mean.

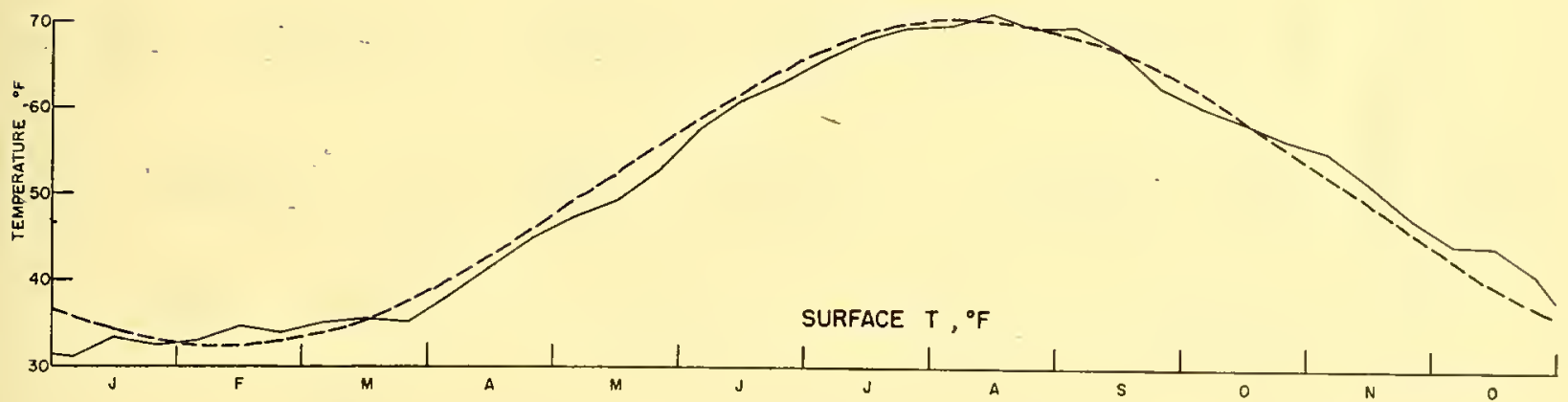

Figure 8. Woods Hole, 1956. (Dashed line, mean for period 1880-1956) 
㪹

نำ

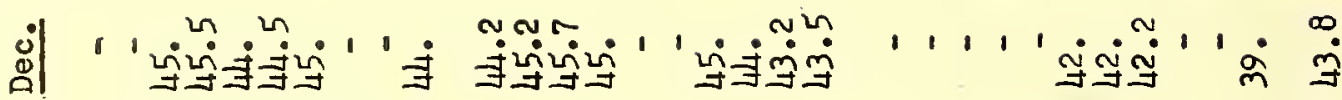

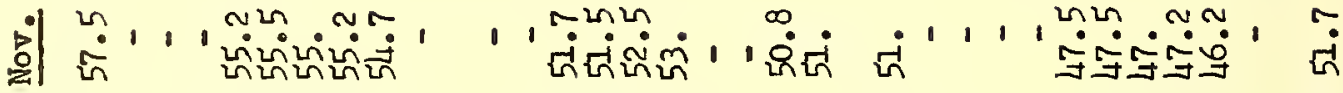

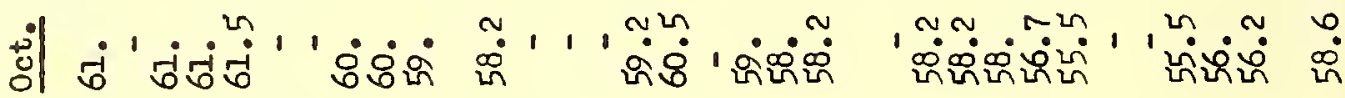

离

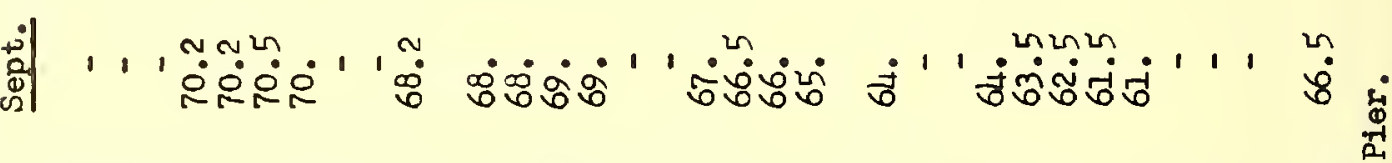

婂

妾

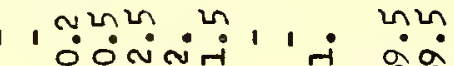

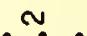

-1. :

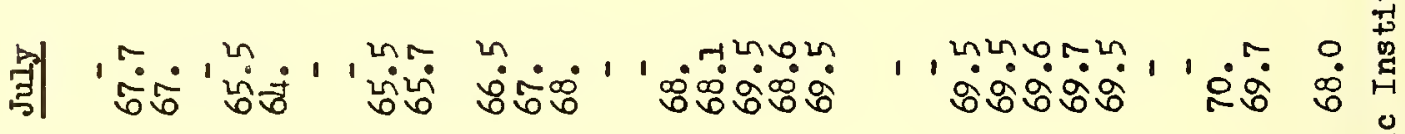

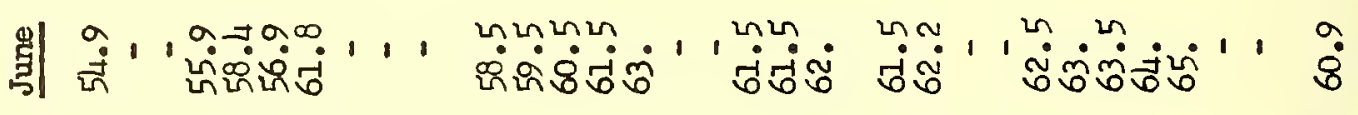

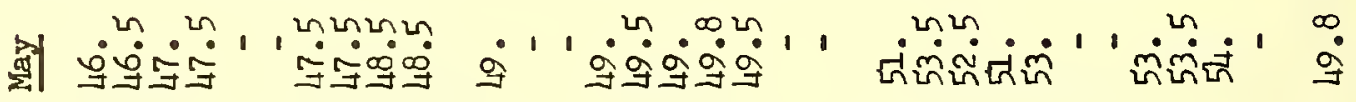

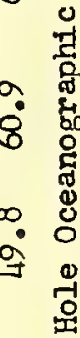

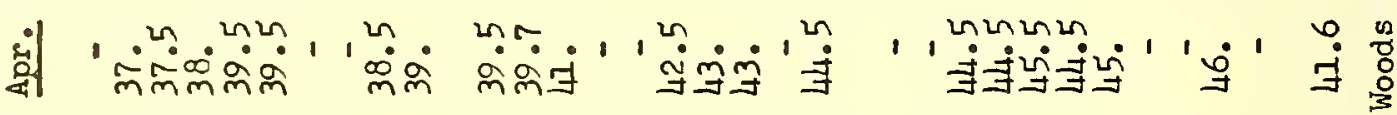

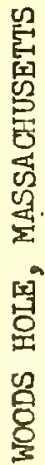

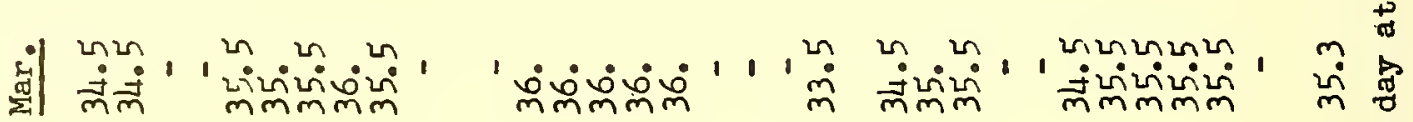

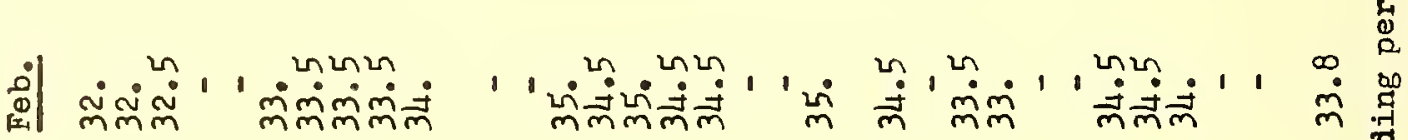

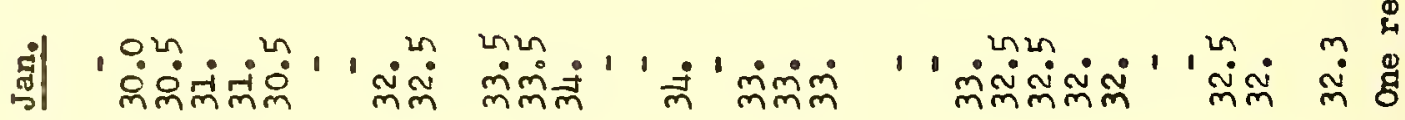

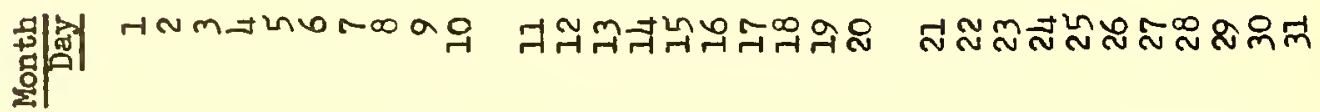




\section{Buzzards Lightship}

This is the first set of data from Buzzards lightship. However, the reader may wish to refer to the fine record from Vineyard Sound Lightship for the period 1878-1910, at a distance of less than four miles (Bumpus, 1957).

The record of surface water temperatures commenced in March with readings in the vicinity of $35^{\circ} \mathrm{F}$, rose to a maximum in late August of $680 \mathrm{~F}$. The temperature decline in the autumn apparently was retarded as mentioned above, inasmuch as the November mean was comparable to the maximum Vineyard Sound Lightship. November mean and the December mean was substantially higher than any Vineyard Sound Lightship December mean for the period 1878-1910.

Surface salinity ranged from a minimum mean for March of $31.6 \%$ to a monthly maximum of 32.3 in December.
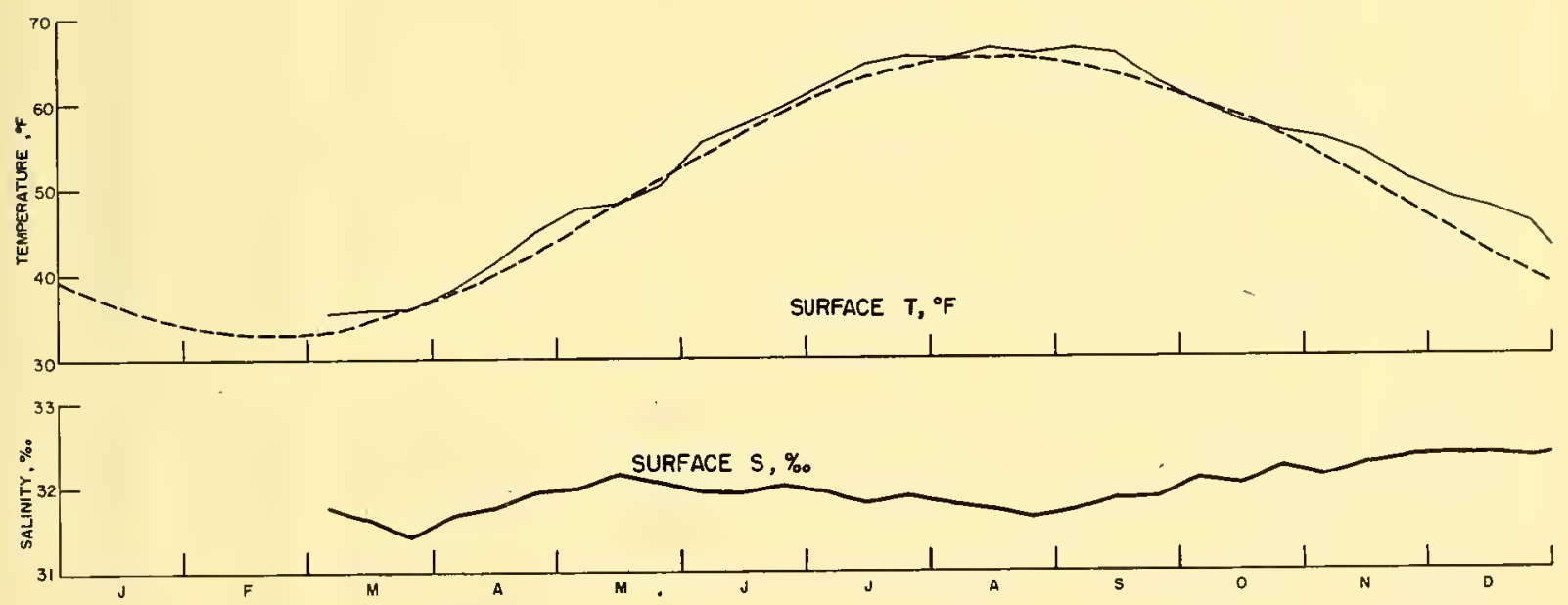

Figure 9. Buzzards Iightship, 1956. (Dashed line, mean for Vineyard Sound Lightship, 1878-1910) 
BUZZARDS LIGHTSHIP $41^{\circ} 24^{\prime} 00^{\prime} \mathrm{N}$. $71^{\circ} 03^{\prime} 00^{\prime} \mathrm{T}$.

Depth of water - 74 feet

\section{Surface Water - 1956}

Month

\begin{tabular}{|c|c|c|c|c|c|c|c|c|c|c|}
\hline$y$ & \multicolumn{2}{|c|}{ March } & \multicolumn{2}{|c|}{ April } & \multicolumn{2}{|c|}{ May } & \multicolumn{2}{|c|}{ June } & \multicolumn{2}{|c|}{ July } \\
\hline & $T^{\circ} F$ & Sal\% & $T^{\circ} \mathrm{F}$ & Sal\% & $\mathrm{T}^{\circ} \mathrm{F}$ & Sal\% & TOF & Sal\% & ${ }^{\infty} \mathrm{F}$ & Sal\% \\
\hline$\frac{1}{2}$ & $\begin{array}{l}34.8 \\
34.8 \\
35.0 \\
35.4 \\
35.7 \\
35.3 \\
35.6 \\
35.7 \\
35.3 \\
35.9\end{array}$ & $\begin{array}{r}31.78 \\
.74 \\
.79 \\
.78 \\
.70 \\
.77 \\
.85 \\
.84 \\
.76 \\
.57\end{array}$ & $\begin{array}{l}7.0 \\
7.8 \\
7.1 \\
7.0 \\
9.0 \\
9.0 \\
8.4\end{array}$ & $\begin{array}{l}1.55 \\
.82 \\
.86 \\
.86 \\
.39 \\
.21 \\
.87 \\
.96 \\
.40 \\
.54\end{array}$ & $\begin{array}{l}45 \\
46 \\
45 \\
54 \\
52 \\
47 \\
48\end{array}$ & $\begin{array}{l}.85 \\
.80 \\
.06 \\
.89 \\
.04 \\
.76 \\
.69 \\
.10\end{array}$ & $\begin{array}{l}54 \\
54 \\
54 \\
56 \\
57 \\
56 \\
55 \\
55 \\
55 \\
56\end{array}$ & $\begin{array}{r}31.93 \\
.82 \\
31.90 \\
32.03 \\
31.72 \\
- \\
32.00 \\
31.91 \\
31.94 \\
32.03\end{array}$ & $\begin{array}{l}62.7 \\
62.9 \\
63.2 \\
61.5 \\
61.3 \\
61.0 \\
61.8 \\
60.8 \\
63.1 \\
61.1\end{array}$ & $\begin{array}{r}.9 \\
.9 \\
31.9 \\
32.0 \\
31.8 \\
.8\end{array}$ \\
\hline 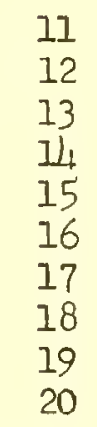 & $\begin{array}{l}35.7 \\
36.4 \\
36.1 \\
36.1 \\
36.2 \\
36.7 \\
35.2 \\
35.8 \\
35.1 \\
35.0\end{array}$ & $\begin{array}{l}.65 \\
.39 \\
.78 \\
.72 \\
.70 \\
.83 \\
.51 \\
.50 \\
.50\end{array}$ & 2 & $\begin{array}{l}.35 \\
.83 \\
.67 \\
.87 \\
.90 \\
.99 \\
.96 \\
.49 \\
.75 \\
.57\end{array}$ & $\begin{array}{l}47 \\
47 \\
48 \\
48 \\
49 \\
50 \\
48 \\
48^{\circ} \\
48 \\
48\end{array}$ & $\begin{array}{r}.05 \\
.29 \\
.20 \\
32.15 \\
31.98 \\
32.23 \\
.32 \\
32.14 \\
31.95 \\
32.06\end{array}$ & $\begin{array}{l}57 * \\
56 \\
58 \\
55 \\
58 \\
61 \\
58 \\
55 \\
57 \\
58\end{array}$ & $\begin{array}{r}32.02 \\
31.92 \\
.85 \\
.72 \\
.68 \\
31.69 \\
32.03 \\
.05 \\
.02 \\
.10\end{array}$ & $\begin{array}{l}65.0 \\
65.5 \\
65.3 \\
64.8 \\
64.0 \\
65.5\end{array}$ & $\begin{array}{l}.8 \\
.8 \\
.7 \\
.7 \\
.8 \\
.8 \\
.80 \\
.80\end{array}$ \\
\hline
\end{tabular}

$\begin{array}{lllllllllll}21 & 35.1 & .27 & 45 & .44 & 49 & 31.94 & 59 & 32.02 & 64.0 & .80\end{array}$

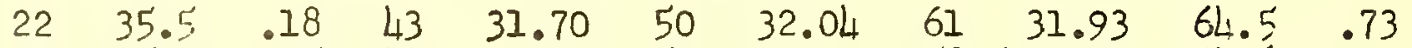

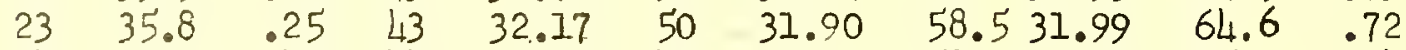

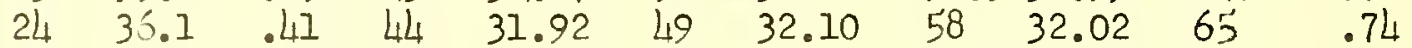

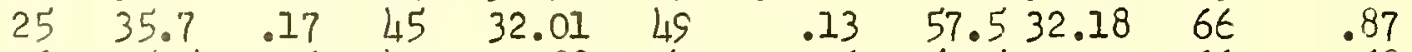

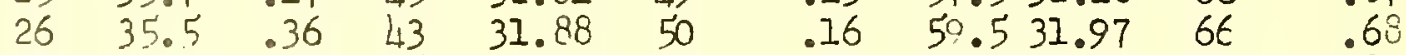

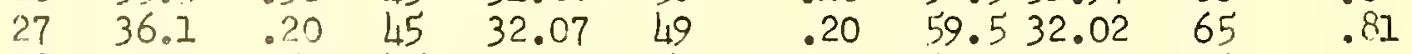

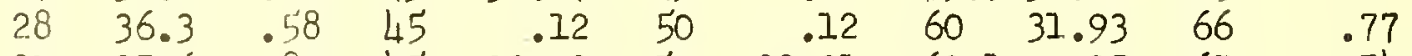

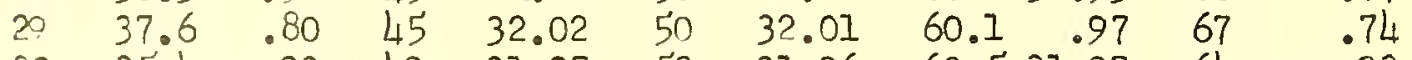

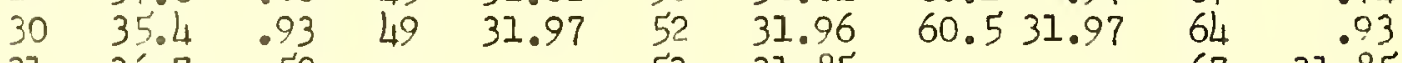

$3136.7 .52-5331.85-5731.85$

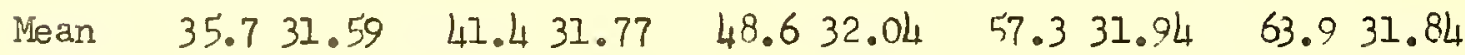

* Moved 11 Jun 500 yds. $220^{\circ}$ from above position to $41^{\circ} 23150^{\prime}$ 'N. $71^{\circ} 03 ' 15$ ' W. 
BUZZARDS LICHTSHIP $47^{\circ} 24^{\prime} 00^{\prime}$ 'N. $77^{\circ} 03^{\prime} 00^{\prime}$ 'W.* Depth of water - 74 feet Surface Water - 1956

Month

\begin{tabular}{|c|c|c|c|c|c|c|c|c|c|c|}
\hline \multirow[t]{2}{*}{ Day } & \multicolumn{2}{|c|}{ August } & \multicolumn{2}{|c|}{ September } & \multicolumn{2}{|c|}{ October } & \multicolumn{2}{|c|}{ Novernber } & \multicolumn{2}{|c|}{ December } \\
\hline & $\mathrm{T}^{\circ} \mathrm{F}$ & Sal\% & TOF & Sal\% & $\mathrm{T}_{\mathrm{F}}$ & Sal\% & $\mathrm{T}^{\mathrm{O}} \mathrm{F}$ & Sal\% & $T^{O} F$ & Sal: \\
\hline $\begin{array}{l}1 \\
2 \\
3 \\
4 \\
5 \\
6 \\
7 \\
8 \\
9 \\
10\end{array}$ & $\begin{array}{l}65 \\
65 \\
64 \\
65 \\
64 \\
64 \\
65 \\
64 \\
68 \\
64\end{array}$ & $\begin{array}{r}31.75 \\
.83 \\
- \\
.86 \\
.82 \\
.87 \\
.70 \\
.82 \\
.78 \\
.76\end{array}$ & $\begin{array}{l}67 \\
67 \\
68 \\
68 \\
65 \\
65 \\
65 \\
66 \\
65 \\
66\end{array}$ & $\begin{array}{r}31.79 \\
.58 \\
.90 \\
.57 \\
.61 \\
.63 \\
.64 \\
.65 \\
.91 \\
.79\end{array}$ & $\begin{array}{l}60 \\
60 \\
62 \\
60 \\
59 \\
59 \\
59 \\
59 \\
60 \\
60\end{array}$ & $\begin{array}{l}31.87 \\
32.00 \\
32.02 \\
32.08 \\
31.97 \\
32.05 \\
31.99 \\
31.97 \\
32.02 \\
31.92\end{array}$ & $\begin{array}{l}55 \\
55 \\
54 \\
54 \\
54 \\
57 \\
57 \\
57 \\
57 \\
55\end{array}$ & $\begin{array}{r}32.18 \\
.11 \\
.18 \\
.09 \\
- \\
.13 \\
.10 \\
32.08 \\
31.99\end{array}$ & $\begin{array}{l}48 \\
48 \\
48 \\
48 \\
48 \\
50 \\
49 \\
50 \\
1.9 \\
48\end{array}$ & $\begin{array}{r}32.03 \\
32.35 \\
31.99 \\
32.53 \\
.02 \\
.61\end{array}$ \\
\hline $\begin{array}{l}11 \\
12 \\
13 \\
14 \\
15 \\
16 \\
17 \\
18 \\
19 \\
20\end{array}$ & $\begin{array}{l}66 \\
65 \\
66 \\
66 \\
67 \\
67 \\
67 \\
67 \\
66\end{array}$ & $\begin{array}{l}.71 \\
.76 \\
.92 \\
.72 \\
.92 \\
.69 \\
.48 \\
.69 \\
.64 \\
.66\end{array}$ & $\begin{array}{l}66 \\
67 \\
67 \\
68 \\
67 \\
64 \\
64 \\
64 \\
64 \\
64\end{array}$ & $\begin{array}{l}.86 \\
.88 \\
.92 \\
.76 \\
.67 \\
.74 \\
.73 \\
.88 \\
.92 \\
.91\end{array}$ & $\begin{array}{l}58 \\
58 \\
56 \\
58 \\
58 \\
59 \\
58 \\
58 \\
58 \\
55\end{array}$ & $\begin{array}{l}31.92 \\
32.00 \\
32.04 \\
31.91 \\
31.88 \\
31.88\end{array}$ & $\begin{array}{l}54 \\
54 \\
54 \\
54 \\
54 \\
55 \\
54 \\
54 \\
53 \\
52\end{array}$ & $\begin{array}{r}32.09 \\
.10 \\
- \\
.09 \\
.23 \\
.49 \\
.15 \\
.30 \\
.30 \\
.16\end{array}$ & $\begin{array}{l}48 \\
48 \\
48 \\
45 \\
48 \\
47 \\
47 \\
47 \\
48 \\
48\end{array}$ & .. \\
\hline $\begin{array}{l}21 \\
22 \\
23 \\
24 \\
25 \\
26 \\
27 \\
28 \\
29 \\
30 \\
31\end{array}$ & $\begin{array}{l}64 \\
64 \\
64 \\
66 \\
61 \\
66 \\
67 \\
68 \\
68 \\
67 \\
67\end{array}$ & $\begin{array}{l}.57 \\
.64 \\
.66 \\
.58 \\
.59 \\
.71 \\
.62 \\
.65 \\
.73 \\
.64 \\
.67\end{array}$ & $\begin{array}{l}64 \\
63 \\
63 \\
63 \\
64 \\
63 \\
61 \\
62 \\
59 \\
60 \\
-\end{array}$ & $\begin{array}{r}.88 \\
.95 \\
31.98 \\
32.01 \\
31.82 \\
.57 \\
.77 \\
.67 \\
.98 \\
31.95 \\
-\end{array}$ & $\begin{array}{l}57 \\
58 \\
60 \\
58 \\
48 \\
55 \\
54 \\
58 \\
59 \\
55 \\
58\end{array}$ & $\begin{array}{l}32.26 \\
32.37 \\
32.21 \\
32.03 \\
32.28 \\
32.21 \\
32.29 \\
32.15 \\
32.12 \\
32.12 \\
32.20\end{array}$ & $\begin{array}{l}54 \\
53 \\
52 \\
50 \\
50 \\
52 \\
52 \\
48 \\
49 \\
48 \\
-\end{array}$ & $\begin{array}{l}.37 \\
.27 \\
.19 \\
.00 \\
.00 \\
.58 \\
.56 \\
.50 \\
.29 \\
.21 \\
.21\end{array}$ & $\begin{array}{l}47 \\
47 \\
47 \\
47 \\
47 \\
46 \\
45 \\
45 \\
45 \\
43 \\
43\end{array}$ & $\begin{array}{l}.2 \\
. ? \\
.7 \\
.6 \\
.3 \\
.3 \\
.1 \\
.2 \\
. ? \\
.1 \\
.2\end{array}$ \\
\hline
\end{tabular}

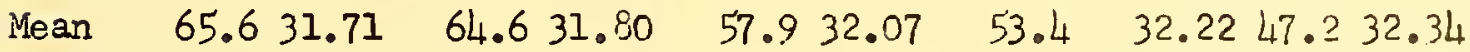

* Moved 11 Jun 500 yds. $220^{\circ}$ from abore position to $41^{\circ} 23^{1} 50^{\prime} \mathrm{N} .71^{\circ} 03^{\prime} 15^{\prime} \mathrm{w}$. 
Kingston, R. I.

Through the kindness of Dr. Steacy Hicks at the Narragansett Marine Laboratory, a temperature record was comenced at the Laboratory Pier on 6 september. This location is within sight of the Brenton Reef Iightship, a location for which we have a particularly fine record for the period 1878-1943.

The mean surface water temperatures for the last quarter 1956 ran a degree to several degrees lower than the Brenton Reef means for the period of record.

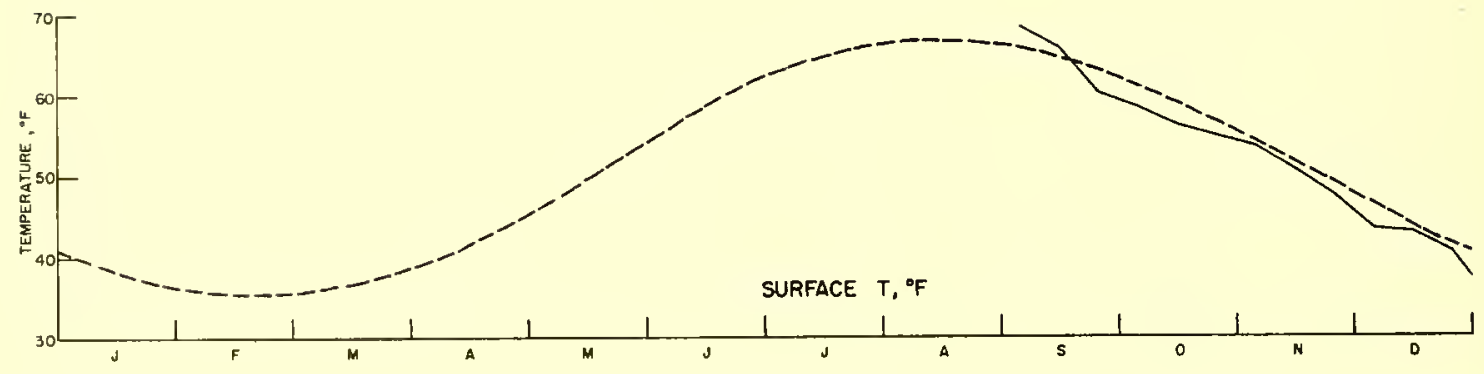

Figure 10. Kingston, R. I., 1956. (Dashed line, mean for Brenton Reef Lightship, 1878-1943) 
Kingston, $R_{\text {. I. }}$

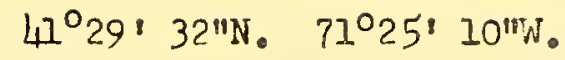

Surface Water Temperature ${ }^{\circ} \mathrm{C}$.

1956

Month

$\overline{\text { Day }}$

September October

November December

Max. Min. Max, Min

Max, Min. Max. Min.

$\begin{array}{rcccccccc}1 & - & - & 15.3 & 14.6 & 13.3 & 12.6 & 7.0 & 6.0 \\ 2 & - & - & 15.2 & 14.2 & 12.9 & 12.8 & 7.0 & 6.0 \\ 3 & - & - & 15.5 & 14.5 & 12.0 & 11.6 & 7.1 & 6.0 \\ 4 & - & - & 15.0 & 14.5 & 12.4 & 11.4 & 7.2 & 6.0 \\ 5 & - & - & 15.0 & 14.5 & 12.1 & 11.6 & 6.2 & 5.4 \\ 6 & 21.9 & 20.2 & 15.0 & 13.6 & 12.1 & 11.4 & 6.9 & 5.6 \\ 7 & 21.4 & 20.0 & 15.0 & 14.4 & 12.1 & 11.3 & 7.4 & 5.0 \\ 8 & 20.6 & 19.2 & 14.9 & 14.1 & 12.3 & 11.7 & 7 \% 0 & 6.1 \\ 9 & 20.0 & 19.2 & 14.6 & 14.0 & 12.0 & 11.3 & 6.1 & 5.4 \\ 10 & 19.9 & 19.0 & 14.2 & 13.5 & 10.4 & 9.6 & 7.0 & 5.0\end{array}$

$\begin{array}{lllll}11 & 19.1 & 18.7 & 13.8 & 13.2\end{array}$

$\begin{array}{llll}10.5 & 9.9 & 7.2 & 5.0\end{array}$

$\begin{array}{lllll}12 & 19.0 & 18.8 & 13.9 & 13.0\end{array}$

$11.0 \quad 10.0 \quad 7.5 \quad 5.4$

$\begin{array}{llll}10.6 & 9.7 & 7.5 & 6.4\end{array}$

$\begin{array}{lllll}13 & 19.9 & 19.2 & 13.2 & 12.4\end{array}$

$\begin{array}{llll}10.9 & 9.6 & 6.9 & 5.5\end{array}$

$\begin{array}{lllll}15 & 19.5 & 18.5 & 14.0 & 13.0\end{array}$

$\begin{array}{llll}11.2 & 10.2 & 5.9 & 5.1\end{array}$

$\begin{array}{lllll}16 & 19.4 & 18.5 & 13.8 & 13.0\end{array}$

$\begin{array}{llll}12.0 & 10.2 & 6.8 & 5.1\end{array}$

$\begin{array}{lllll}17 & 18.6 & 18.4 & 14.4 & 13.3\end{array}$

$\begin{array}{lllll}18 & 18.6 & 18.0 & 13.9 & 13.5\end{array}$

$\begin{array}{llll}17.4 & 10.2 & 7.0 & 5.2\end{array}$

$\begin{array}{lllll}19 & 18.5 & 17.0 & 13.6 & 12.9\end{array}$

$\begin{array}{llll}10.5 & 9.7 & 7.0 & 5.2\end{array}$

$\begin{array}{lllll}20 & 17.6 & 16.8 & 13.6 & 12.2\end{array}$

$\begin{array}{llll}10.1 & 9.3 & 6.5 & 4.6\end{array}$

$21 \quad \begin{array}{lllll}17.4 & 16.2 & 14.0 & 12.2\end{array}$

$\begin{array}{llll}10.0 & 9.1 & 5.9 & 5.4\end{array}$

$22 \quad 16.0 \quad 15.6 \quad 14.0 \quad 13.1$

$\begin{array}{lllll}23 & 17.1 & 15.7 & 13.6 & 13.4\end{array}$

$\begin{array}{lllll}24 & 16.8 & 16.0 & 13.9 & 13.5\end{array}$

$\begin{array}{lllll}25 & 16.5 & 15.5 & 13.2 & 12.1\end{array}$

$\begin{array}{lllll}26 & 16.0 & 15.3 & 12.3 & 11.6\end{array}$

$\begin{array}{lllll}27 & 15.4 & 14.6 & 12.6 & 11.4\end{array}$

$\begin{array}{lllll}28 & 14.8 & 14.0 & 12.3 & 11.7\end{array}$

$\begin{array}{lllll}29 & 14.6 & 13.7 & 12.5 & 11.6\end{array}$

$\begin{array}{lllll}30 & 15.5 & 14.5 & 12.5 & 11.6\end{array}$

31 - $\quad 12.911 .8$

$\begin{array}{llll}10.9 & 9.3 & 6.3 & 5.4\end{array}$

$\begin{array}{llll}10.9 & 10.2 & 5.9 & 5.1\end{array}$

$\begin{array}{llll}10.2 & 8.9 & 5.5 & 4.4\end{array}$

$\begin{array}{llll}9.4 & 8.0 & 5.0 & 4.0\end{array}$

$\begin{array}{llll}8.8 & 7.8 & 5.7 & 3.8\end{array}$

$8.4 \quad 7.5 \quad 5.4 \quad 3.6$

$\begin{array}{llll}8.0 & 6.8 & 5.4 & 4.9\end{array}$

$8.0 \quad 6.9 \quad 5.4 \quad 4.0$

$8.0 \quad 7.0 \quad 5.4 \quad 4.0$

$\begin{array}{llll}8.0 & 6.2 & 5.1 & 2.9\end{array}$

$\begin{array}{lllllllll}\text { Mean } & 18.2 & 17.3 & 13.9 & 13.1 & 10.7 & 9.7 & 6.3 & 5.0\end{array}$

$\begin{array}{llllr}{ }^{\circ} \mathrm{C} & 17.7 & 13.5 & 10.2 & 5.6 \\ { }^{\circ} \mathrm{F} & 63.9 & 56.3 & 50.4 & 42.1\end{array}$

* Narragansett Marine Laboratory Pier. 
Ambrose Lightship

Surface water temperatures during the spring months tended to $\mathrm{lag}$ behind the means for the period of record (1937-1955) (Bumpus, 1957), were equivalent during the summer, commenced to cool off more quickly in late September and early October, then more slowly in late October and November.

Minimum temperatures throughout the whole water column were observed during the last third of March at less than $38^{\circ} \mathrm{F}$. The thermocline commenced development in April. Maximal temperatures at the surface of $720 \mathrm{~F}$ occurred during the first third of September and at the bottom of $59^{\circ} \mathrm{F}$ during the last third of October and first two-thirds of November.

The surface salinity ranged from a maximum of $32.4 \%$ at the beginning of the year to $26.0 \%$ during mid-April and gradually recovered to $32.0 \%$ at the end of the year. Bottom salinities tended to be several parts per thousand salter than surface water except during the fall overturn in October and November. 

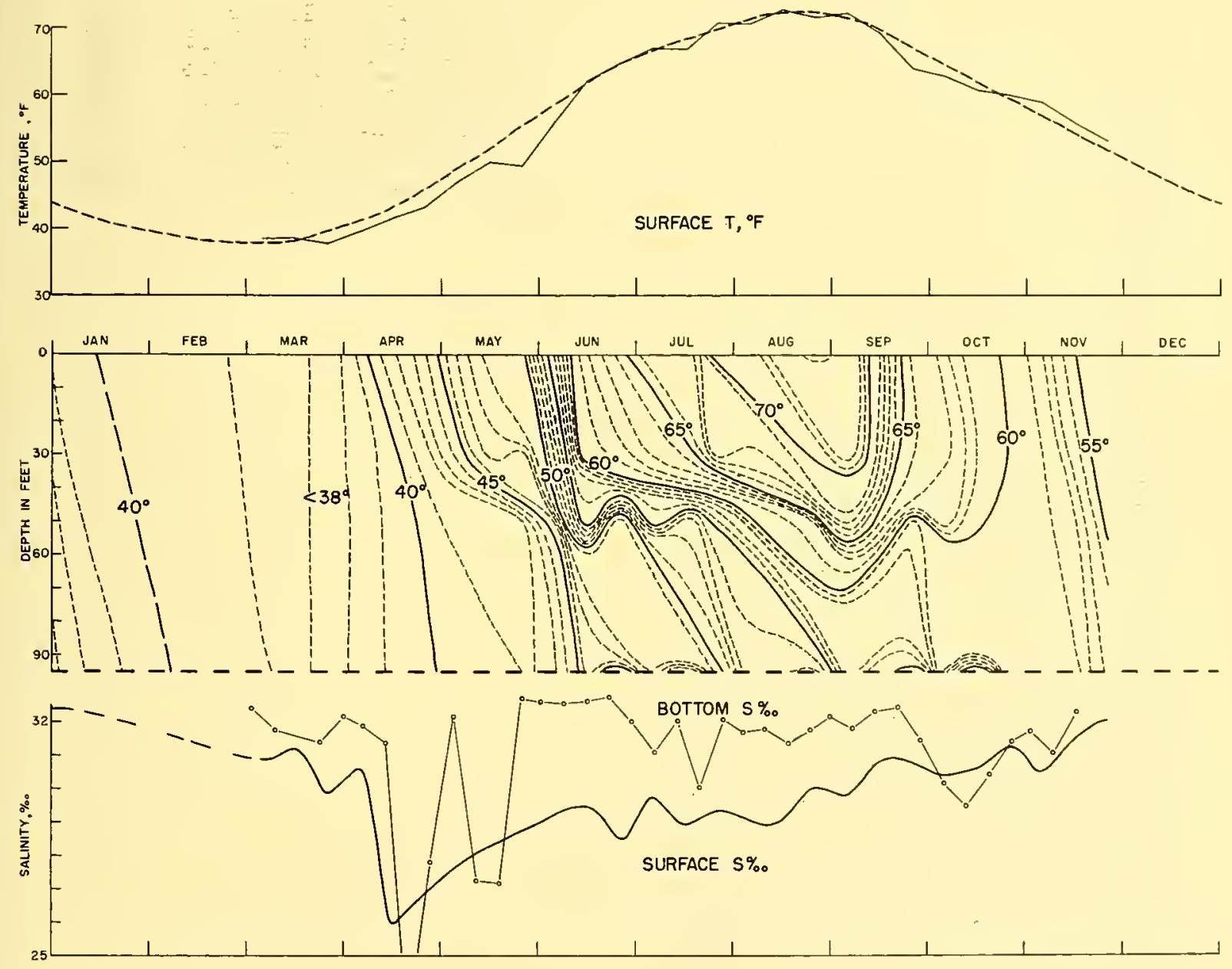
Figure 11. Ambrose Lightship, 1956. (Dashed line in upper diagram, mean for period 
AMBROSE LIGHTSHIP

1955

December

Temperature ${ }^{\circ} \mathrm{F}$

Dept

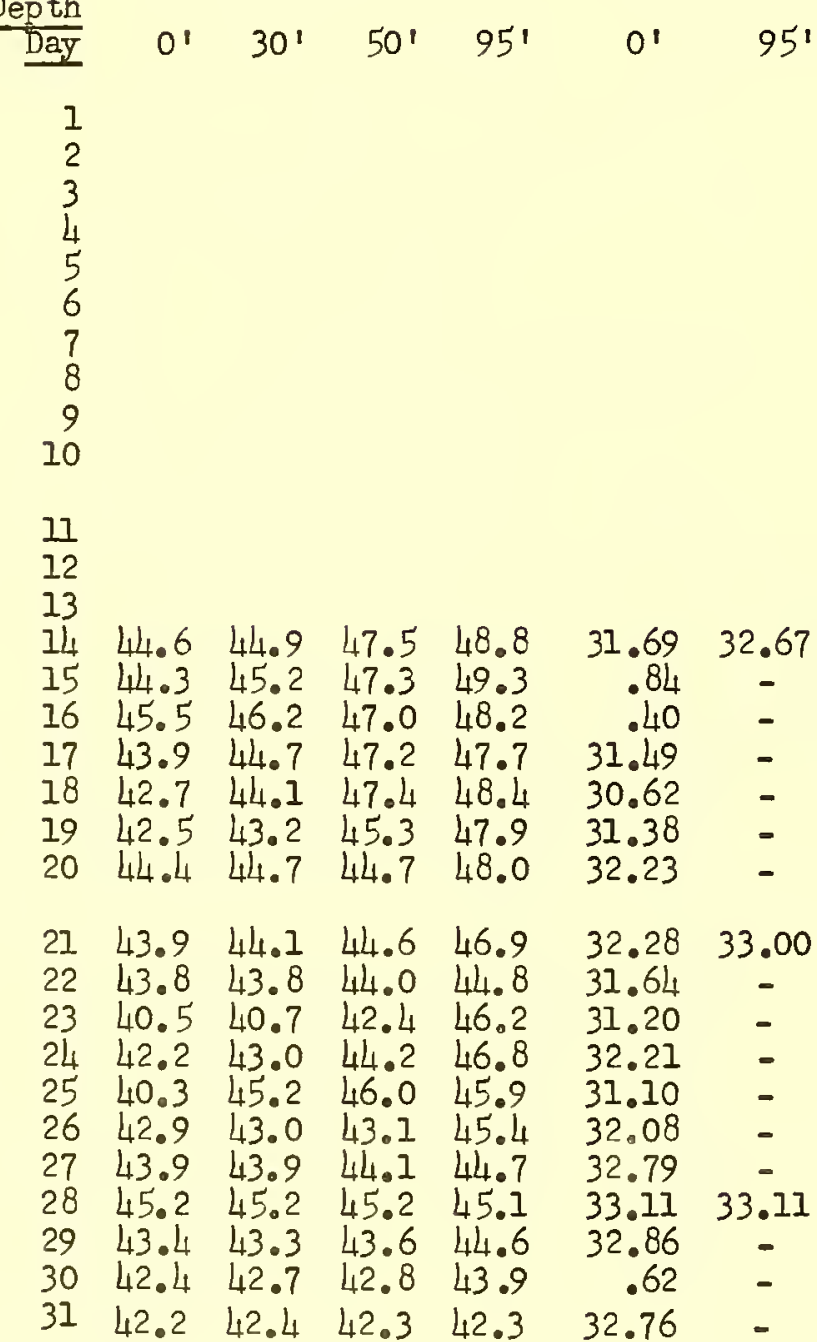

$\begin{array}{llllll}\text { Mean } \quad 43.3 \quad 43.9 & 44.9 & 46.4 & 31.96 & 32.92\end{array}$
Depth of water - 95 feet 1956

January

Temperature $\stackrel{\circ}{F}$ Salinity ${ }^{\circ} / 00$ $\begin{array}{lllll}41.0 & 40.5 & 41.3 & 43.3 & 31.96\end{array}$

$\begin{array}{lllll}40.7 & 40.7 & 41.3 & 42.1 & 32.30\end{array}$

$40.8 \quad 41.7 \quad 42.4 \quad 42.8$

$40.3 \quad 40.9 \quad 41.4 \quad 42.6$

$41.141 .1 \quad 41.1 \quad 42.2$

$41.6 \quad 41.6 \quad 41.6 \quad 41.7$

$\begin{array}{lllll}40.7 & 40.7 & 40.7 & 40.7 & 32.67\end{array}$ 


\section{6}

Temperature ${ }^{\circ} \mathrm{F}$ Salinity $/ 00$ Temperature ${ }_{\mathrm{F}}^{\text {March }}$ Salinity $/ 00$

Depth

Day $0^{\prime} \quad 30^{\prime} \quad 50^{\prime} \quad 95^{\prime} \quad 0^{\prime} \quad 95^{\prime} \quad 0^{\prime} \quad 30^{\prime} \quad 50^{\prime} 95^{\prime} \quad 0^{\prime} \quad 95^{\prime}$

$\begin{array}{lllll}1 & 37.6 & 38.0 & 38.0 & 39.1\end{array}$

$\begin{array}{cc}- & - \\ - & 32.41 \\ - & - \\ 29.40 & - \\ 31.99 & - \\ - & - \\ 31.67 & - \\ 30.47 & 31.77 \\ .81 & -\end{array}$

38.

$\begin{array}{lllll}3 & 38.0 & 37.4 & 37.9 & 39.3\end{array}$

$\begin{array}{lllll}4 & 37.7 & 36.9 & 38.1 & 38.2\end{array}$

$\begin{array}{lllll}5 & 38.8 & 38.8 & 39.0 & 39.6\end{array}$

$\begin{array}{lllll}6 & 38.7 & 38.7 & 38.7 & 39.1\end{array}$

$\begin{array}{lllll}7 & 38.8 & 38.5 & 39.0 & 39.5\end{array}$

$\begin{array}{lllll}8 & 39.0 & 39.0 & 39.1 & 39.1\end{array}$

$\begin{array}{lllll}9 & 38.8 & 38.7 & 38.8 & 38.8\end{array}$

$\begin{array}{lllll}10 & 38.8 & 38.9 & 39.1 & 39.2\end{array}$

$\begin{array}{lllll}11 & 39.8 & 39.4 & 39.0 & 39.8\end{array}$

. .59

$-$

$\begin{array}{lllll}12 & 39.7 & 39.5 & 39.3 & 40.0\end{array}$

30.89
31.00

1439.

$38.8 \quad 38.9$.

$31 . \overline{10}$

$-$

\section{5}

$-\quad-30.64$

$\begin{array}{llll}38.0 & 37.7 & 38.0 & 29.46\end{array}$

$\begin{array}{lllll}38.5 & 38.2 & 37.7 & 38.1 & 31.01\end{array}$

$\begin{array}{lcccc}40.5 & 39.0 & 37.9 & 37.9 & 29.12\end{array}$

$\begin{array}{lllll}42.7 & 38.2 & 37.9 & 37.9\end{array}$

$\begin{array}{lllll}39.0 & 38.8 & 37.3 & 37.3 & 30.33\end{array}$

$\begin{array}{lllll}38.9 & 38.8 & 38.9 & 39.0 & 31.36\end{array}$

$\begin{array}{lllll}39.0 & 38.8 & 39.0 & 39.0 & .33\end{array}$

$\begin{array}{lllll}39.7 & 39.3 & 39.0 & 38.9 & 31.59\end{array}$

$\begin{array}{lllll}40.5 & 39.3 & 38.8 & 38.8 & 30.29\end{array}$

$40 . \quad-\quad-30.31$

$40.839 .539 .7 \quad-28.72$

$\begin{array}{lllll}41.6 & 39.2 & 39.1 & 39.0 & 28.19\end{array}$

31.36

$\begin{array}{lllll}15 & 39.0 & 38.5 & 39.0 & 39.1\end{array}$

$\begin{array}{lllll}16 & 38.8 & 38.8 & 38.8 & 39.1\end{array}$

$\begin{array}{lllll}17 & 38.3 & 38.3 & 38.3 & 38.0\end{array}$

.51

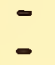

41.5

42.0

39.6

$\begin{array}{lll}39.0 & 39.0 & 23.79\end{array}$

42.3

39.8

$42.0 \quad 39.9$

$39.0-25.40$

$.46=$

$\begin{array}{lllll}19 & 37.5 & 37.5 & 37.5 & 37.4\end{array}$

.28

42.240.

39.5

$39.5 \quad 23.83$

42.5 -

$39.5 \quad 39.5$

23.49

$\begin{array}{lllll}20 & 37.3 & 37.2 & 37.2 & 37.3\end{array}$

$.49 \quad-$

$\begin{array}{lllll}21 & 37.2 & 37.2 & 37.2 & 37.2\end{array}$

$\begin{array}{lllll}22 & 37.5 & 37.3 & 37.2 & 37.2\end{array}$

$\begin{array}{lllll}23 & 37.8 & 37.1 & 37.1 & 37.3\end{array}$

$\begin{array}{lllll}24 & 37.8 & 37.2 & 37.1 & 37.1\end{array}$

$\begin{array}{lllll}25 & 36.8 & 36.8 & 37.2 & 37.4\end{array}$

$\begin{array}{lllll}26 & 37.2 & 37.2 & 37.2 & 38.0\end{array}$

$\begin{array}{lllll}27 & 37.5 & 37.0 & 37.5 & 37.8\end{array}$

$\begin{array}{rc}.50 & - \\ .44 & - \\ 31.11 & 31.39 \\ 26.68 & - \\ 31.28 & = \\ 29.58 & - \\ .74 & - \\ .78 & - \\ .06 & - \\ .06 & 32.14 \\ 29.10 & -\end{array}$

$\begin{array}{lllll}41.5 & 40.6 & 39.4 & 39.5 & 28.70\end{array}$

$\begin{array}{lllll}42.5 & 40.3 & 39.6 & 39.6 & 23.51\end{array}$

43.5

42.2

$\begin{array}{llll}40.3 & 40.0 & 40.2 & 26.91\end{array}$

$\begin{array}{lllll}42.5 & 41.7 & 40.1 & 40.1 & 27.35\end{array}$

$\begin{array}{lllll}42.7 & 42.3 & 40.8 & 40.1 & 27.02\end{array}$

$\begin{array}{lllll}42.1 & 41.7 & 40.2 & 40.1 & 27.49\end{array}$

$43.242 .040 .5-27.76$

$46.041 .840 .6-26.94$

$\begin{array}{lllll}29 & 38.2 & 38.1 & 38.0 & 38.7\end{array}$

$\begin{array}{lllll}30 & 38.2 & 37.6 & 37.8 & 38.2\end{array}$

$\begin{array}{lllll}31 & 38.2 & 38.0 & 38.0 & 38.3\end{array}$ 45.0

$47.9 \quad 40.5$

$-$

27.65

$\begin{array}{lllllll}\text { Mean } & 38.2 & 38.0 & 38.1 & 38.4 & 30.54 & 31.93\end{array}$

$\begin{array}{llllll}41.4 & 40.0 & 39.2 & 39.0 & 27.70 & 28.46\end{array}$ 


\title{
1956
}

\author{
May \\ Temperature ${ }^{\circ} \mathrm{F}$ Salinity $\stackrel{\circ}{100}$ \\ Temperature ${ }^{\circ} \stackrel{\text { June }}{\text { Salinity }} \stackrel{0}{\circ}$
}

Depth

\begin{tabular}{|c|c|c|c|c|c|c|c|c|c|c|c|c|}
\hline Day & 01 & $30^{\prime}$ & $50^{\prime}$ & $95^{\circ}$ & $0^{\prime}$ & $95^{1}$ & $0^{\prime}$ & $30^{\prime}$ & 50.1 & $95^{\prime}$ & $0^{\prime}$ & 951 \\
\hline 1 & 46.6 & 43.2 & 41.4 & 0.5 & 28.83 & - & 53.0 & 46.8 & 2 & 1.2 & 26.74 & 32.61 \\
\hline 2 & 47.5 & 44.2 & 40.7 & 0.4 & 3 & - & 51.6 & 46.0 & 1.4 & & 20. & - \\
\hline 3 & 47.0 & 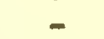 & - & 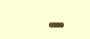 & 28.51 & - & 54.0 & 50.2 & 17.4 & .2 & 27. & - \\
\hline$\overline{4}$ & 47.5 & 44.7 & 40.8 & 40.9 & 29.51 & 32.17 & 56.6 & 51.4 & 3 & & 29.40 & - \\
\hline 5 & 47.1 & 45.0 & 41.0 & 40.7 & 29.45 & - & 57.3 & 53.4 & 42.4 & .9 & 30.06 & - \\
\hline 6 & 46.8 & 44.6 & 41.6 & 40.8 & 5.23 & - & 55.8 & 50 & 46.0 & .4 & 30.48 & - \\
\hline 7 & 47.6 & 44.4 & 40.5 & 40.9 & 8.76 & - & 57.6 & 52 & 48.0 & & 29.15 & - \\
\hline 8 & 46.6 & 45.6 & 42.3 & 40.8 & 29.93 & - & 56.5 & 52.6 & 50.8 & & 29.48 & 32.59 \\
\hline 9 & 46.9 & 46.0 & 42.6 & 40.9 & 8.63 & - & 58.0 & $5 F_{4}$ & 50.4 & .4 & 30.34 & - \\
\hline 10 & 46.4 & 45.5 & 42.0 & 40. & 27.30 & - & 0 & 57 & 兵.0 & .0 & & - \\
\hline 11 & 47 & - & - & - & 27.24 & 7.25 & 58.7 & 57.9 & 52.5 & & & - \\
\hline 12 & 47.5 & & 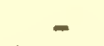 & & 28. & - & & 57 & 52.2 & & 30. & - \\
\hline 13 & 50.0 & 43.1 & 41.3 & 40.8 & 27.31 & - & & 60.0 & 5 & & 29 & $=$ \\
\hline 14 & 51.5 & 42.6 & 40.8 & 40.9 & 27. & - & 6 & 59 & 53.7 & & 29. & - \\
\hline 15 & 51.5 & & & & 3 & - & & 60 & 56.5 & & 28. & 31.64 \\
\hline 16 & 51.5 & 50 & 44.2 & 47.7 & 26 & - & 8 & 62 & 59.6 & & 26. & - \\
\hline 1 & 49. & 49.5 & 43.0 & 41. & 29.50 & - & 63.5 & 60.0 & 56.2 & 45.1 & 29. & - \\
\hline 1 & 49. & 49.2 & 41.3 & 40. & 27.01 & 27.1 & 65 & - & - & - & 30. & - \\
\hline 19 & 51. & 48.8 & 41.1 & 40. & 29. & - & 62.8 & 62.5 & 55.1 & & 30. & - \\
\hline 20 & 48.2 & 46.6 & 41.5 & 40.7 & 30.07 & - & 62.0 & 60.2 & 55.6 & 47.5 & 28. & - \\
\hline 21 & 50.3 & 46.8 & 41.4 & 40. & 27. & - & 63.5 & 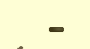 & & - & .67 & - \\
\hline 22 & 51.3 & 46.9 & 40.8 & 40. & 27 & - & 63.6 & 60.7 & 47.7 & 45.0 & .2 & 31.74 \\
\hline 23 & 48.0 & 46.2 & 41.1 & 40.8 & 30 & - & & 61.3 & 45.2 & 42.8 & & - \\
\hline 24 & 48.5 & - & - & - & 27.01 & - & 62.4 & 62.3 & 54.0 & 43.2 & 28.08 & - \\
\hline 2 & 46.5 & & & - & 30.31 & 32.73 & & 61.3 & 48.4 & 44.9 & 29.04 & - \\
\hline 28 & 48.2 & 45.0 & 41.2 & - & 28.45 & - & & 61.8 & 51.8 & 45.0 & 29.28 & - \\
\hline$?$ & 49.0 & 46.0 & 41.6 & 41.0 & 28.77 & - & 65 & $=$ & & & 27.81 & - \\
\hline & 50 & - & - & & 29.51 & 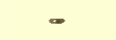 & & 55 & 46. & 44.5 & 28.38 & 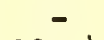 \\
\hline & 48.5 & 47.5 & 41.3 & 41.3 & 30.98 & 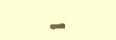 & 64 & 62. & 48. & & 28.80 & 2.04 \\
\hline & 50.5 & 47.7 & 47.2 & 47.4 & 27.93 & - & 65.8 & 67.0 & 49.4 & 43.2 & 27.84 & \\
\hline & & & 4 & 41.1 & 26 & $=$ & & & & & & \\
\hline
\end{tabular}

$\begin{array}{lllllll}\text { Mean } & 48.7 & 46.0 & 41.5 & 40.9 & 28.21 & 29.84\end{array}$

$\begin{array}{llllll}60.8 & 57.3 & 49.7 \quad 44.4 & 29.06 & 32.12\end{array}$ 


\section{6}

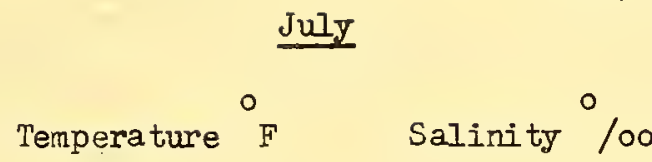

\author{
August \\ Temperature ${ }^{\circ} \mathrm{F}$ \\ Salinity 100
}

Depth

Day

$\begin{array}{lllllll}1 & 68.9 & 60.1 & 48.2 & 44.4 & 28.45 & -\end{array}$

$\begin{array}{lllllll}2 & 65.6 & 60.0 & 47.9 & 43.6 & 27.93\end{array}$

$\begin{array}{lllllll}3 & 67.6 & 58.1 & 48.9 & 43.5 & 29.76 & -\end{array}$

$\begin{array}{llllll}4 & 66.0 & - & - & - & 30.81 \\ 5 & 66.9 & 65.1 & 52.9 & 44.0 & 30.95=\end{array}$

$\begin{array}{llllll}6 & 64.9 & 64.9 & 64.8 & 52.3 & 30.92\end{array}$

$\begin{array}{lllll}7 & 67.3 & 67.0 & 66.9 & 51.8\end{array}$

$\begin{array}{lllll}8 & 68.0 & - & - & -\end{array}$

30.33

31.08

29.66

$\begin{array}{rrrrrrr}9 & 67.1 & 65.5 & 56.8 & 53.4 & .35 & . \\ 10 & 67.0 & 65.8 & 54.2 & 51.0 & .15 & -\end{array}$

$\begin{array}{llllll}11 & 63.8 & 63.5 & 54.3 & 48.3 & 29.75\end{array}$

$\begin{array}{lllll}12 & 65.4 & 64.5 & 54.1 & 45.4\end{array}$

$\begin{array}{lllll}13 & 65.7 & 65.4 & 54.0 & 45.0\end{array}$

$\begin{array}{lllll}14 & 67.8 & 67.5 & 56.2 & 46.6\end{array}$

$\begin{array}{lllll}15 & 65.8 & 65.1 & 51.2 & 45.0\end{array}$

$\begin{array}{lllll}16 & 67.2 & 62.8 & 50.9 & 43.3\end{array}$

$\begin{array}{lllll}17 & 67.4 & 62.2 & 53.0 & 44.1\end{array}$

$\begin{array}{lllll}18 & 66.9 & 60.0 & 52.1 & 44.5\end{array}$

$\begin{array}{lllll}19 & 68.6 & 66.9 & 51.5 & 46.0 \\ 20 & 69.7 & 64.4 & 50.6 & 46.3\end{array}$

28.99

$29.01 \quad 32.06$

$.71=$

$29.30-$

$.48=$

$28.75=$

29.01

$27.79 \quad 30.04$

$69.869 .052 .2-30.63=$

$\begin{array}{llllll}22 & 68.9 & 68.8 & 68.6 & 52.1 & .71\end{array}$

$\begin{array}{lllll}23 & 70.2 & 68.9 & 59.9 & 52.2\end{array}$

$\begin{array}{llllll}24 & 70.5 & 69.8 & 54.9 & 50.9 & 30.17\end{array}$

$\begin{array}{lllllll}25 & 69.5 & 68.8 & 52.1 & 48.1 & 28.71 & -\end{array}$

$\begin{array}{lllllll}26 & 69.8 & 68.1 & 52.3 & 47.9 & .50 & -\end{array}$

$\begin{array}{lllllll}27 & 69.8 & 69.7 & 53.3 & 48.7 & .69 & 32.07\end{array}$

$28 \quad 74.5$

2975.

$\begin{array}{lllll}30 & 70.9 & 70.0 & 52.4 & 48.4\end{array}$

$\begin{array}{lllll}31 & 68.9 & 66.0 & 52.9 & 49.0\end{array}$

$.07 \quad-$

$28.95-$

29.61 -

$28.12-$

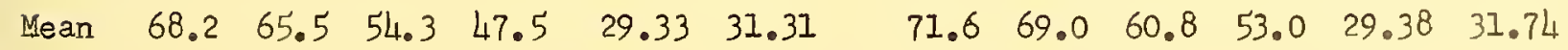


AMBROSE LIGHTSHIP

September

Temperature $F$ $40^{\circ} 27.1 \cdot N .73^{\circ} 49.4 \mathrm{~W}$.

Depth of water - 95 feet
1956

0

Salinity $/ 00$

$0^{\prime} \quad 95^{\prime}$

$\begin{array}{lllll}62.7 & 63.0 & 63.8 & 62.2 & 30.39\end{array}$

$\begin{array}{lllll}63.1 & 63.2 & 63.4 & 63.0 & 31.12\end{array}$

$\begin{array}{lllll}62.8 & 63.8 & 63.7 & 62.8 & 29.79\end{array}$

$62.363 .963 .3-29.30$

$\begin{array}{lllll}62.9 & 63.7 & 63.5 & 56.9 & 29.56\end{array}$

$\begin{array}{lllll}62.5 & 62.7 & 61.6 & 54.9 & 30.87\end{array}$

$63.263 .563 .4-30.59$

$\begin{array}{lllll}62.1 & 62.6 & 63.0 & 60.0 & 30.42\end{array}$

$\begin{array}{lllll}63.0 & 63.0 & 62.8 & 56.2 & 30.75\end{array}$

$62.062 .058 .0-30.84$

$\begin{array}{llllll}10 & 70.2 & 70.3 & 70.1 & - & 31.00\end{array}$

$\begin{array}{llllll}11 & 68.4 & 70.1 & 70.0 & 62.1 & 29.96\end{array}$

$\begin{array}{llllll}12 & 69.8 & 69.7 & 68.3 & 56.1 & 30.86\end{array}$

$\begin{array}{llllll}13 & 69.3 & 69.2 & 55.9 & 52.9 & 30.60\end{array}$

$\begin{array}{llllll}14 & 69.5 & 68.9 & 59.0 & 52.2 & 31.01 \\ 15 & 69.7 & 68.0 & 54.0 & 51.8 & 30.95\end{array}$

$1669.6-31.05$

$\begin{array}{llllll}17 & 69.1 & 69.0 & 68.9 & 58.0 & 30.93\end{array}$

$\begin{array}{llllll}18 & 69.2 & 69.1 & 69.1 & 59.1 & 31.05\end{array}$

19

20

2

\section{$\begin{array}{lllll}68.1 & 68.1 & 68.0 & - & -\end{array}$}

$\begin{array}{llll}66.2 & 66.5 & 65.9 & 49.4\end{array}$

\section{$\begin{array}{lllll}63.9 & 66.1 & 65.8 & 48.2\end{array}$}

$\begin{array}{lllll}65.0 & 66.0 & 55.1 & 48.1\end{array}$

$\begin{array}{lllll}62.9 & 65.0 & 47.3 \quad 47.1\end{array}$

$61.3 \quad 56.9 \quad 47.1 \quad 47.0$

$\begin{array}{llll}64.0 & 63.9 & 53.5 & 48.5\end{array}$

$\begin{array}{llll}64.6 & 64.9 & 64.8 & 64.7\end{array}$

$\begin{array}{llll}63.8 & 63.9 & 63.9 & 63.9\end{array}$

63.063 .0

63.0

63.0

30.85

29.75

30.03

29.99

30.71

31.20

31.36

31.34

31.56

63.0

63.1

63.1

62.8
$61.4 \quad 61.4 \quad 58.8 \quad 52.0 \quad 31.06$

$61.0 \quad 61.0 \quad 60.1 \quad 53.9 \quad 31.05$

$61.0 \quad 61.0 \quad 60.7 \quad 56.4 \quad 31.12$

$\begin{array}{lllll}60.9 & 60.9 & 61.1 & 57.0 & 31.48\end{array}$

$\begin{array}{llllll}60.8 & 60.7 & 60.2 & 55.8 & 30.95\end{array}$

$\begin{array}{lllll}61.0 & 61.6 & 61.5 & 53.1 & 29.27\end{array}$

$\begin{array}{lllll}60.8 & 61.0 & 60.9 & 54.9 & 30.74\end{array}$

$\begin{array}{lllll}59.9 & 60.2 & 59.3 & 52.9 & 29.54\end{array}$

$60.260 .9-\quad-30.47$

$\begin{array}{lllll}59.8 & 60.8 & 60.7 & 57.8 & 30.41\end{array}$

$60.4 \quad 61.0 \quad 60.7 \quad 58.4 \quad 30.61$

$\begin{array}{lllll}60.8 & 60.9 & 60.9 & - & 30.82\end{array}$

$\begin{array}{lllll}60.9 & 60.9 & 60.8 & 59.3 & 31.18\end{array}$

$\begin{array}{lllll}61.5 & 61.2 & 60.7 & 59.4 & 31.09\end{array}$

$\begin{array}{lllll}58.5 & 58.9 & 59.0 & 58.6 & 31.30\end{array}$

$\begin{array}{llllll}59.5 & 59.9 & 59.9 & 59.9 & 31.34 & 31.44\end{array}$

$59.659 .659 .7-31.30$

$\begin{array}{lllll}59.3 & 59.3 & 59.3 & 59.4 & 31.61\end{array}$

$\begin{array}{lllll}59.0 & 59.1 & 59.1 & 59.0 & 31.36\end{array}$

$59.259 .259 .2 \quad-31.37$

$\begin{array}{lllll}59.8 & 59.7 & 59.5 & 59.6 & 31.36\end{array}$
29.49

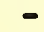

.

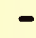

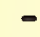

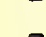

30.41

$-$

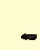

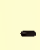

$-$

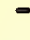

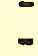

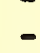

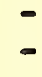

$\begin{array}{lllllllllllll}\text { Mean } & 68.3 & 68.4 & 64.1 & 55.7 & 30.46 & 32.00 & 60.9 & 61.3 & 61.0 & 57.6 & 30.74 & 30.38\end{array}$ 
Table 10 (cont'd)

AMBROSE LIGHTSHIP

1956

November

Temperature $\stackrel{\circ}{F} \quad$ Salinity ${ }^{\circ} / 00$

Depth

Day $0^{\prime} \quad 30^{\prime} 50^{\prime} 95^{\prime} \quad 0^{\prime} 95^{\prime}$

$\begin{array}{llllll}1 & 59.1 & 59.1 & 59.1 & 59.0 & 29.72\end{array}$

$\begin{array}{lllllll}2 & 59.2 & 59.8 & 59.7 & 59.5 & 29.42 & 31.74\end{array}$

$\begin{array}{llllllll}3 & 59.2 & 59.1 & 59.1 & 59.1 & 21.54 & -\end{array}$

$\begin{array}{lllllll}4 & 58.5 & - & - & - & .43 & - \\ 5 & 59.3 & 59.4 & 59.5 & 59.7 & .43\end{array}$

$\begin{array}{llllll}6 & 59.2 & 59.2 & 59.3 & 59.4 & 31.49\end{array}$

$\begin{array}{lllllll}7 & 59.1 & 59.4 & 59.5 & 59.4 & 29.66 & -\end{array}$

$\begin{array}{lllllll}8 & 58.5 & - & - & - & 28.55 & - \\ 9 & 58.4 & 58.8 & 59.4 & 58.5 & 31.12 & 31.08\end{array}$

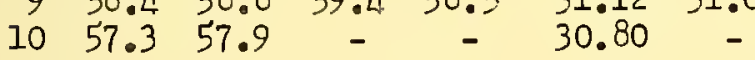

$11 \quad 56.7 \quad 57.6 \quad 58.0 \quad 58.3 \quad 02 \quad-$

$\begin{array}{cccccc}12 & 57.0 & 57.4 & 58.0 & 58.6 & .98 \\ 13 & 56.3 & 56.8 & - & - & 30.64\end{array}$

1456.256 .3 - -31.23 -

$\begin{array}{lllllll}15 & 54 . & - & - & - & 31.27 & - \\ 16 & 57.3 & 57.3 & 58.9 & 59.5 & 32.28 & 32.30\end{array}$

$\begin{array}{lllllll}16 & 57.3 & 57.3 & 58.9 & 59.5 & 32.28 & 32.30\end{array}$

$\begin{array}{lllllll}18 & 55.2 & 56.3 & 58.0 & 60.9 & -\end{array}$

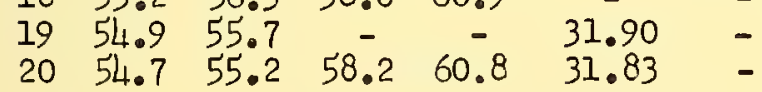

$\begin{array}{lllllll}21 & 55.3 & 55.6 & 58.1 & 60.0 & 30.82 & \text { - }\end{array}$

$2252.0-32.38$ -

$\begin{array}{llllll}23 & 54.8 & 55.7 & 56.3 & - & .47\end{array}$

$24 \quad 53.9 \quad 54.7 \quad 54.9 \quad 59.0 \quad .51$ -

$25 \quad 54.154 .154 .559 .5 \quad .51$ -

$\begin{array}{llllll}26 & 53.3 & 53.3 & 54.0 & 54.9 & .06\end{array}$

$\begin{array}{llllll}27 & 53.4 & 53.4 & 54.9 & 54.9 & 32.59\end{array}$

$\begin{array}{llllll}28 & 52.3 & 53.0 & 53.9 & 54.5 & 31.60\end{array}$

$\begin{array}{lllllll}29 & 51.7 & 53.4 & - & - & .71 & -\end{array}$

$\begin{array}{lllllll}30 & 50.3 & 51.1 & 53.0 & 56.1 & 31.71 & -\end{array}$

31

$\begin{array}{llllll}\text { Mean } \quad 55.9 & 56.4 & 57.5 & 58.6 & 31.32 & 31.71\end{array}$ 
Minimum surface temperature of $38^{\circ} \mathrm{F}$ occurred during the last third of Jamary. Surface temperatures remained less than $40^{\circ}$ until during the first third of April. The vernal warming larged behind the mean for the period of record 1947-1956 until the end of June. Maximum temperatures of $750 \mathrm{~F}$ were observed during the first third of September followed by autumnal cooling virtually identical with the mean for the period of record.

The thermocline commenced development in April, reached its maximum in July. Maximum temperature at the bottom of nearly $64^{\circ} \mathrm{F}$ was reached in early October when the autumn overturn reached the bottom following which the temperature in the water column was essentially isothermal for the remainder of the year.

The surface salinity commenced at the beginning of the year at slightly above $32 \%$, sradually descended to a minimum of just over $30 \%$ during mid-eTune and gradually recovered to just under $32 \%$ by year end. Bottom salinities remained, for the most part, in the vicinity of $32 \%$. Surface and bottom salinities took an unexplained jump in the last third of October to $33^{\circ} / 00$.
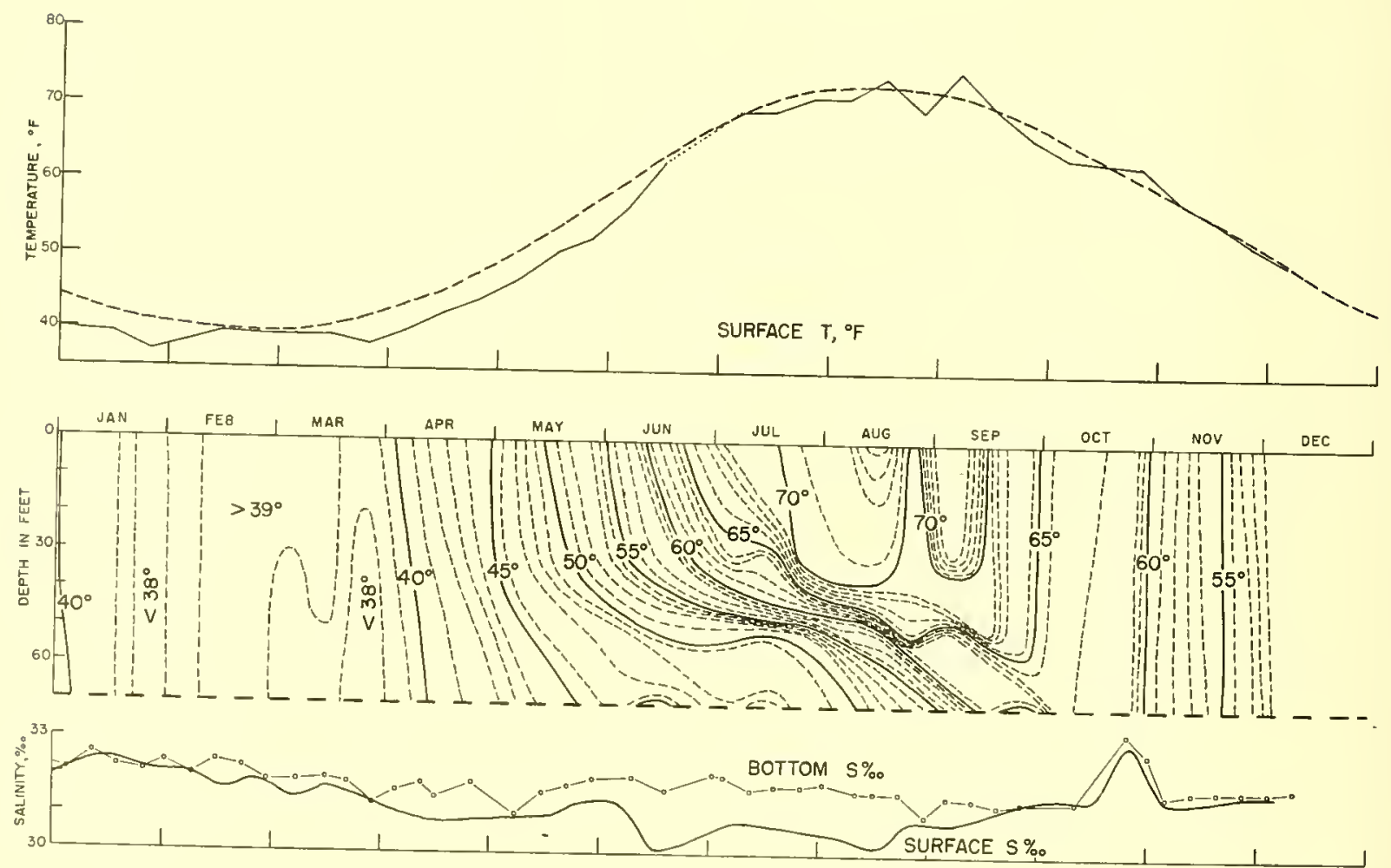
Figure 12. Barnejat Iightship, 1956. (Dashed line in upper diasram, mean for period
1947-1956) 


\begin{tabular}{|c|c|c|}
\hline & & Dece \\
\hline mperature ${ }^{\circ} \mathrm{F}$ & Salinity $\%$ & Temperature ${ }^{\circ} \mathrm{F}$ \\
\hline
\end{tabular}

$\underline{\text { Depth }}$

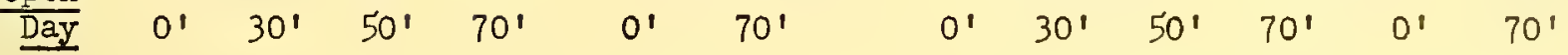

$\begin{array}{llllll}44.9 & 44.8 & 44.8 & 44.8 & 31.16 & 31.22\end{array}$

$\begin{array}{llllll}44.4 & 44.5 & 45.1 & 45.1 & .10 & -\end{array}$ $44.2 \quad 44.2 \quad 4.2 \quad 44.2 \quad .31$ $44.144 .1 \quad 44.144 .1 \quad .46=$ $\begin{array}{llllll}41.5 & 42.4 & 42.8 & 43.9 & .06 & -\end{array}$

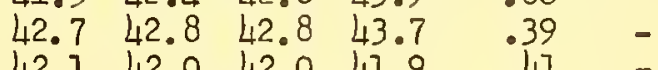


BARNEGAT LIGHTSHIP

$$
\text { Temperature }{ }^{O_{F}}
$$

$\frac{\text { Depth }}{\text { Day }} \quad 0^{\prime} \quad 30^{\prime} \quad 50^{\prime} 70^{\prime} \quad 0^{\prime} 70^{\prime}$

$\begin{array}{llllll}1 & 37.6 & 37.6 & 37.6 & 38.2 & 31.71\end{array}$

$\begin{array}{llllll}2 & 38.3 & 38.3 & 38.7 & 39.1 & 32.02\end{array}$

$\begin{array}{lllll}3 & 37.4 & 37.5 & 38.2 & 39.1\end{array}$

4 43. - - -

$\begin{array}{llllll}6 & 41.2 & 41.3 & 41.7 & 41.8 & 32.64\end{array}$

$\begin{array}{lllll}7 & 41.2 & 41.3 & 41.7 & 41.9\end{array}$

$\begin{array}{lllll}8 & - & - & - & -\end{array}$

10

$\begin{array}{lllll}11 & 40.9 & 40.8 & 40.8 & 40.8\end{array}$

12

13

14

15

16

17

$\begin{array}{lllll}18 & 38.1 & 38.1 & 38.7 & 38.7\end{array}$

$\begin{array}{lllll}19 & 37.9 & 37.9 & 37.9 & 37.9\end{array}$

$\begin{array}{lllll}20 & 37.9 & 38.0 & 38.0 & 38.0\end{array}$

$\begin{array}{lllll}21 & 37.7 & 37.7 & 37.7 & 37.7\end{array}$

$\begin{array}{lllll}22 & 37.7 & 37.7 & 37.7 & 37.7\end{array}$

$\begin{array}{lllll}23 & 36.7 & 36.7 & 37.8 & 37.8\end{array}$

$\begin{array}{lllll}24 & 37.2 & 37.2 & 37.2 & 37.0\end{array}$

$\begin{array}{lllll}25 & 37.1 & 37.1 & 37.1 & 37.1\end{array}$

$\begin{array}{lllll}26 & 37.3 & 37.3 & 37.3 & 37.3\end{array}$

$\begin{array}{lllll}27 & 35.4 & 35.4 & 35.5 & 35.5\end{array}$

$\begin{array}{lllll}28 & 37.9 & 37.9 & 37.9 & 37.9\end{array}$

29

$\begin{array}{lllllll}30 & 36.5 & 36.6 & 38.0 & 38.3 & 31.84 & - \\ 31 & 37.6 & 37.0 & 37.0 & 38.0 & 32.14 & -\end{array}$

$\begin{array}{lllllll}\text { Mean } & 38.6 & 38.3 & 38.5 & 38.7 & 32.25 & 32.28\end{array}$

\section{February}

Temperature ${ }^{\circ} F \quad$ Salinity $\%$

$0^{\prime} \quad 30^{\prime} \quad 50^{\prime} \quad 70^{\prime} \quad 0^{\prime} \quad 70^{\prime}$

$\begin{array}{llllll}37.7 & 37.7 & 37.7 & 38.3 & 32.10 & 32.35\end{array}$

$\begin{array}{llllll}37.9 & 37.7 & 38.0 & 38.4 & .19 & -\end{array}$

$\begin{array}{llllll}37.5 & 37.9 & 37.5 & 38.1 & .04 & -\end{array}$

$\begin{array}{llllll}38.0 & 38.0 & 38.0 & 38.2 & .24 & -\end{array}$

$\begin{array}{lllll}38.2 & 38.2 & 38.2 & 38.2 & .21\end{array}$

$\begin{array}{llllll}38.1 & 38.0 & 38.0 & 38.1 & .16 & -\end{array}$

$\begin{array}{llllll}38.8 & 38.5 & 38.2 & 38.5 & 32.14 & - \\ 38.5 & 38.3 & 38.2 & 38.3 & 31.97 & 32.05\end{array}$

$\begin{array}{lllllll}38.9 & 38.9 & 38.9 & 38.9 & .98 & =\end{array}$

$\begin{array}{lllll}39.2 & 39.0 & 39.0 & 39.0 & 31.95\end{array}$

$\begin{array}{llllllll}.60 & 32.59 & 39.2 & 39.2 & 39.2 & 39.1 & 32.19 & -\end{array}$

$\begin{array}{llllll}39.2 & 39.2 & 39.2 & 39.1 & 32.19 & - \\ 39.9 & 39.5 & 39.3 & 39.2 & 32.02 & -\end{array}$

$\begin{array}{llllll}39.5 & 39.9 & 39.7 & 39.3 & 31.70 & -\end{array}$

$\begin{array}{lllll}39.0 & 38.8 & 39.0 & 39.0 & 31.60\end{array}$

$\begin{array}{llllll}39.9 & 39.7 & 39.8 & 40.0 & 29.84 & 32.43\end{array}$

$\begin{array}{lllllll}40.1 & 40.0 & 39.8 & 40.1 & 31.93 & =\end{array}$

$\begin{array}{lllll}39.9 & 39.9 & 39.9 & 39.9 & 32.00\end{array}$

$40.0 \quad 40.0 \quad 40.0 \quad 40.0 \quad 31.85 \quad-$

$\begin{array}{lllll}40.0 & 40.0 & 40.0 & 39.9 & 32.03\end{array}$

$\begin{array}{lllll}39.8 & 40.1 & 40.1 & 40.2 & 31.71\end{array}$

$\begin{array}{lllll}39.8 & 39.9 & 40.1 & 40.2 & 31.89\end{array}$

$40.0 \quad 40.0 \quad 40.0 \quad 39.8 \quad 32.22 \quad 32.28$

$\begin{array}{lllllll}39.1 & 39.3 & 39.0 & 39.1 & .27 & \text {. }\end{array}$

$\begin{array}{llllllll}.08 & - & 39.1 & 39.0 & 39.0 & 39.4 & 32.48 & -\end{array}$

$\begin{array}{llllll}38.8 & 38.9 & 38.9 & 38.9 & 31.05 & -\end{array}$

$\begin{array}{llllll}39.0 & 39.0 & 39.0 & 39.0 & 31.14 & -\end{array}$

$\begin{array}{llllll}\overline{39.3} & 39.3 & 39.2 & 39.2 & 31.94 & 31.92\end{array}$

$\begin{array}{llllll}39.1 & 39.1 & 39.0 & 39.1 & 31.88 & 32.20\end{array}$ 
Day $0^{\prime} \quad 30^{\prime} 50^{\prime} 70^{\prime} \quad 0^{\prime} 70^{\prime}$

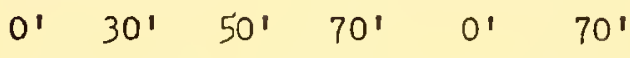

$\begin{array}{lllllll}1 & 39.2 & 39.0 & 38.9 & 38.8 & 32.09 & -\end{array}$

$2 \begin{array}{llllll}2 & 38.5 & 38.6 & 38.5 & 38.4 & 31.61\end{array}$

$\begin{array}{lllllll}3 & 39.4 & 38.5 & 38.2 & 38.4 & 30.95 & - \\ 4 & 39.0 & 39.0 & 38.9 & 38.8 & 31.62 & -\end{array}$

$540 . \quad-\quad-31.24$ -

$\begin{array}{lllllll}6 & 40.0 & 39.4 & 39.2 & 39.1 & 31.86 & 31.94\end{array}$

$\begin{array}{lllllll}8 & 39.0 & 39.1 & 39.1 & 39.0 & 31.93 & -\end{array}$

$\begin{array}{llllll}9 & 39.0 & 39.0 & 38.9 & 38.9 & 30.97\end{array}$

$\begin{array}{llllll}10 & 40.0 & 39.0 & 39.0 & 39.0 & 30.86\end{array}$

$\begin{array}{lllllll}11 & 39.8 & 39.6 & 39.1 & 39.0 & 31.24 & -\end{array}$

$\begin{array}{lllllll}12 & 40.1 & 40.0 & 39.1 & 39.2 & 31.45 & -\end{array}$

$\begin{array}{lllllll}13 & 39.5 & 39.3 & 39.2 & 39.2 & 32.08 & -\end{array}$

$\begin{array}{lllllll}14 & - & - & - & - & - & -\end{array}$

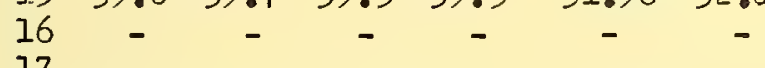

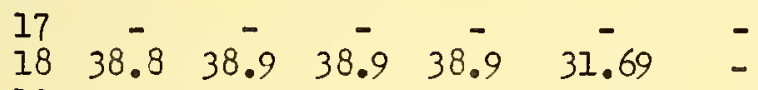

$\begin{array}{lcccccc}19 & - & - & - & - & - & - \\ 20 & 37.9 & 37.9 & 37.9 & 37.9 & 31.92 & -\end{array}$

$2137.038 .8 \quad-\quad-\quad 31.8731 .88$

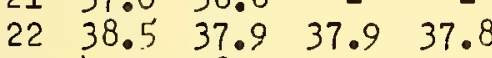

$\begin{array}{lllll}23 & 40.0 & 38.0 & 37.9 & 37.9\end{array}$

$\begin{array}{lcccc}24 & - & - & - & - \\ 25 & 37.9 & 37.5 & 37.8 & 37.8\end{array}$

$\begin{array}{lllll}25 & 37.9 & 37.5 & 37.8 & 37.8 \\ 26 & 38.0 & 37.5 & 37.8 & 37.8\end{array}$

$27 \quad 38.8 \quad 38.0 \quad 37.8 \quad 37.8$

$\begin{array}{lllll}28 & 38.0 & 38.0 & 38.0 & 38.0\end{array}$

$\begin{array}{lllll}29 & 37.2 & 37.2 & 37.2 & 37.2\end{array}$

$\begin{array}{lllll}30 & 38.3 & 38.0 & 38.0 & 37.8\end{array}$

$\begin{array}{lllll}31 & 38.1 & 38.0 & 37.9 & 37.9\end{array}$ $\begin{array}{rl}31.87 & 31.88 \\ .82 & -\end{array}$

$.33-$

$.30 \quad-$

.28 -

. 31 31. 30

$.32=$
$.26=$
$31.29=$ $\begin{array}{llllll}39.5 & 38.0 & 38.0 & 37.9 & 31.28 & -\end{array}$

$\begin{array}{llllll}38.8 & 38.4 & 38.0 & 37.9 & 31.04 & -\end{array}$

$\begin{array}{lllll}39.5 & 39.0 & 38.2 & 38.0 & 30.96\end{array}$

$\begin{array}{llllll}40.9 & 39.0 & 38.0 & 38.0 & .61 & 31.68\end{array}$

$\begin{array}{llllll}40.0 & 39.5 & 38.0 & 38.0 & .75 & \text { - }\end{array}$

$\begin{array}{llllll}41.1 & 39.9 & 38.1 & 38.1 & 30.65 & -\end{array}$

- - - - -

$\begin{array}{llllll}39.8 & 39.7 & 39.5 & 39.5 & 31.77 & -\end{array}$

$\begin{array}{llllll}117.0 & 39.9 & 39.5 & 39.4 & .78\end{array}$

$\begin{array}{llllll}40.0 & 40.1 & 40.0 & 39.4 & 31.82 & 31.86\end{array}$

43.4 $40 \overline{-} \quad 40 \overline{2} \quad 40 \overline{2} \quad 29.71=$

$\begin{array}{llllll}42.9 & 41.1 & 40.9 & 40.8 & 28.35 & 31.52\end{array}$

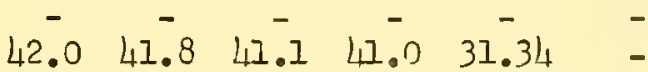

$\begin{array}{lllll}42.0 & 41.6 & 40.8 & 40.8 & .38\end{array}$

44. - - - .37 -

$\begin{array}{lllll}43.4 & 42.7 \quad 41.0 & 40.9 & 31.83\end{array}$

$45.0042 .5 \quad 47.4 \quad 47.0 \quad 30.01$ -

$\begin{array}{llllll}43.2 & 42.4 & 41.2 & 41.1 & 31.19 & -\end{array}$

$\begin{array}{llllrr}43 . & - & - & - & 30.84 & - \\ 43.3 & 42.0 & 41.1 & 41.1 & .99 & 31.86\end{array}$

$\begin{array}{llllll}43.7 & 43.6 & 41.4 & 41.3 & .75 & =\end{array}$

$\begin{array}{lllll}44.5 & 44.0 & 41.5 \quad 41.2 & 30.75 & -\end{array}$

$44.5 \quad 44.0 \quad 41.5 \quad 41.131 .24 \quad-$

$\begin{array}{llllll}46.0 & 44_{.1}^{-} & 42.8 & 41.8 & 31.23 & -\end{array}$

$\begin{array}{llllll}42.2 & 41.1 & 40.1 & 39.9 & 30.94 & 31.73\end{array}$ 
Table 11 (cont'd)

BARINGAT LIGHTSHIP

May

Temperature ${ }^{\circ} \mathrm{F}$

$\frac{\text { Depth }}{\text { Day }} \quad 0^{\prime} \quad 30^{\prime} \quad 50^{\prime} 70^{\prime} \quad 0^{\prime} 70^{\prime}$

1

2

3

4

6

$47.3 \quad 47.7 \quad 41.9 \quad 41.9$

$\begin{array}{llll}47.8 & 47.4 & 43.1 & 42.1\end{array}$

$46.0 \quad 45.9 \quad 45.3 \quad 47.3$

31.00

$\begin{array}{lllll}46.5 & 46.4 & 46.1 & 44.0 & 29.79\end{array}$

$\begin{array}{llllll}10 & 46.5 & 46.2 & 46.0 & 43.2 & 31.34\end{array}$

\section{1}

1

1

15

16

17

18

19

20

$21 \quad 52.5 \quad 52.0 \quad 44.7 \quad 44.4$

$\begin{array}{lllll}22 & 53.0 & 52.5 & 51.0 & 44.1\end{array}$

$\begin{array}{lllll}23 & 52.8 & 52.6 & 44.4 & 44.1\end{array}$

$24 \quad 53.1 \quad 53.0 \quad 44.2 \quad 44.2$

$\begin{array}{lllll}25 & 53.0 & 53.0 & 45.0 & 44.2\end{array}$

$\begin{array}{lllll}2.6 & 51.5 & 51.7 & 44.5 & 44.3\end{array}$

$\begin{array}{lllll}27 & 52.5 & 52.5 & 50.8 & 44.0\end{array}$

$\begin{array}{lllll}28 & 51.5 & 49.4 & 46.3 & 46.4\end{array}$

$\begin{array}{lllll}29 & 52.4 & 51.9 & 47.0 & 46.4\end{array}$

$\begin{array}{llll}30 & 51.5 & 51.5 & 51.0 \quad 47.0\end{array}$

$3151.0 \quad 50.0 \quad 48.0 \quad 46.9$

Mean $39^{\circ} 45.8$ N. $75^{\circ} 56.0$ w.

Depth of water - 72 feet

1956

June

Temperature ${ }^{\circ} \mathrm{F}$

Salinity $\%$

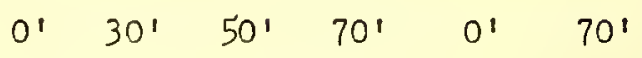

$53.2 \quad 51.0 \quad 47.8 \quad 46.9 \quad 31.58 \quad-$

$\begin{array}{llllll}54.2 & 53.4 & 47.1 & 46.9 & .29 & - \\ 55.3 & 54.0 & 48.3 & 47.0 & .49 & -\end{array}$

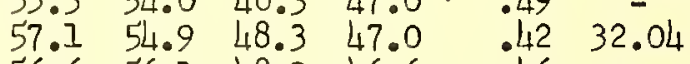

$\begin{array}{llllll}56.6 & 56.1 & 48.9 & 46.6 & .46 & - \\ 58.0 & 56.6 & 47.3 & 46.6 & .46 & -\end{array}$

$\begin{array}{lllll}58.0 & 55.2 & 47.2 & 47.0 & 31.44\end{array}$

$\begin{array}{llllll}58.8 & 54.2 & 47.0 & 46.9 & 30.25 & =\end{array}$

$\begin{array}{lllll}57.8 & 55.2 & 47.7 & 47.0 & 31.51\end{array}$

$\begin{array}{llllll}58.1 & 57.9 & 56.8 & 47.4 & 31.56 & -\end{array}$

$\begin{array}{llllll}58.1 & 57.3 & 48.1 & 47.5 & 30.48 & -\end{array}$

$60.9 \quad 51.0 \quad 46.9 \quad 45.0 \quad 29.65$ -

$\begin{array}{llllll}62.8 & 53.0 & - & - & 30.10 & - \\ 61.2 & 55.0 & 45.8 & 45.9 & 31.00 & -\end{array}$

$\begin{array}{lllll}65.2 & 58.6 & 46.8 & 43.9 & 29.74\end{array}$

$\begin{array}{llllll}62.1 & 55.4 & 48.0 & 40.0 & 29.42 & -\end{array}$

$\begin{array}{llllll}64.9 & 59.6 & 55.0 & 44.5 & 28.86 & \text {.. }\end{array}$

$\begin{array}{llllll}64.9 & 62.0 & 56.0 & 44.8 & 30.76 & 31.68\end{array}$

$\begin{array}{lccccc}62 . & - & - & - & .96 & - \\ 62.9 & 63.5 & 53.9 & 45.1 & .25 & -\end{array}$

$\begin{array}{rrrrrr}65.1 & 63.1 & 51.7 & 44.2 & .10 & - \\ 64.3 & 63.7 & 49.9 & 44.8 & 30.48 & -\end{array}$ 
BARNEGA T LICHTSHIP

$$
39^{\circ} 45.8 \mathrm{~N} .75^{\circ} 56.0 \mathrm{w} \text {. }
$$

Depth of water -72 feet

$$
1956
$$

July

Temperature ${ }^{\circ} \mathrm{F}$

Salinity $\%$

Depth

Day $0^{\prime} \quad 30^{\prime} \quad 50^{\prime} 70^{\prime} \quad 0^{\prime} 70^{\prime}$

$\begin{array}{lllllll}1 & 68.6 & 61.0 & 46.8 & 45.1 & 30.73 & 32.12\end{array}$

$\begin{array}{lllllll}2 & 67.2 & 54.7 & 49.1 & 46.0 & 31.06\end{array}$

$\begin{array}{lllll}3 & 69.3 & 56.4 & 47.2 & 46.0\end{array}$

$\begin{array}{lllll}4 & 70.9 & 67.0 & 47.0 & 46.0\end{array}$

$\begin{array}{ll}30.93 & - \\ 30.91 & 32.08\end{array}$

5

6

\section{(8)}

Temperature ${ }^{\circ}$ F Salinity $\%$

Temperature ${ }^{\circ}$ F Salinity $\%$

\author{
August
}

$\begin{array}{llllll}71.5 & 71.4 & 49.8 & 48.7 & - & .57\end{array}$

\section{0}

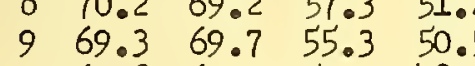

\section{1}

12

\section{il}

15

$15 \quad 68.5 \quad 59.7 \quad 47.7 \quad 46$.

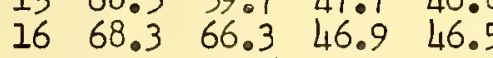

$17 \quad 69.6 \quad 62.5 \quad 46.946 .5$

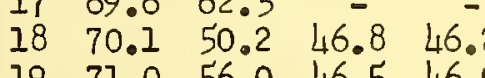

$1971.0 \quad 56.0 \quad 46.5 \quad 46.0$

$\begin{array}{lllll}20 & 70.1 & 60.2 & 47.6 & 46.2\end{array}$

$\begin{array}{lccccc}21 & - & - & - & - & - \\ 22 & 66.1 & 65.7 & 49.0 & 48.2 & .97 \\ 23 & 70.2 & 68.3 & 50.7 & 48.9 & .91\end{array}$

$\begin{array}{lllllll}24 & 70.3 & 68.7 & 49.7 & 48.8 & -\end{array}$

25

26

27

28

30

30

$\begin{array}{lllll}72.2 & 72.2 & 49.7 & 49.0\end{array}$

$50.2 \quad 48.4$

$.95 \quad 31.76$

$\begin{array}{llll}72.2 & 70.4 & 50.8 & 48.6\end{array}$

$.64-$

$52.4 \quad 47.6$

$.51 \quad$

73.072 .5

48.047 .6

.57

.48

30.73

Mean $0^{\prime} 30^{\prime} 50^{\prime} 70^{\prime} \quad 0^{\prime} 70^{\prime}$

$\begin{array}{llllll}70.6 & 70.2 & 47.5 & 46.9 & 30.54 & 31.88\end{array}$

$\begin{array}{llllll}72.0 & 71.3 & 50.0 & 49.0 & .66 & -\end{array}$

$\begin{array}{cccccc}- & - & - & - & - & - \\ - & - & - & - & - & - \\ - & - & - & - & - & - \\ 70.3 & 70.0 & 69.8 & - & -.84 & - \\ 71.7 & 70.8 & 69.0 & - & .39 & - \\ 72.1 & 71.5 & 66.8 & 52.1 & 30.31 & 30.66\end{array}$

$\begin{array}{llllll}72.5 & 70.8 & 67.8 & 52.0 & 29.92 & -\end{array}$

$\begin{array}{cccccc}-72.8 & 71.0 & 65.0 & 51.2 & 29.79= \\ .0 & -\end{array}$

74. $-\quad-\quad 30.13-$

$\begin{array}{llllll}75.9 & 72.3 & 54.3 & 51.1 & .13 & 31.67\end{array}$

$74.1 \quad 72.3 \quad 54.2 \quad 51.1 \quad .16$ -

$\begin{array}{llllll}74.8 & 70.6 & 53.0 & - & .19 & -\end{array}$

$\begin{array}{llllll}74.7 & 72.0 & 51.3 & 51.1 & .21 & -\end{array}$

$\begin{array}{cccccc}75.8 & - & - & - & .61 & - \\ 72.8 & 72.8 & 57.7 & 52.3 & .69 & -\end{array}$

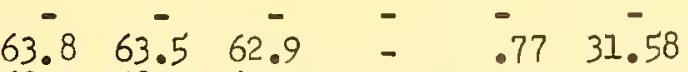

$68.368 .167 .0 \quad-\quad .91$ -

$\begin{array}{llllll}69.8 & 69.7 & 69.2 & 53.0 & 30.99 & -\end{array}$

$70.5-3-5-31.04=$

$70.570 .369 .4-30.91=$

$70.270 .069 .1-.93=$

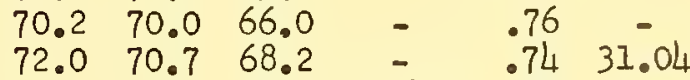

$\begin{array}{lllllll}71.0 & 70.2 & 63.6 & 54.4 & .81 & -\end{array}$

$\begin{array}{llllll}71.5 & 70.3 & 66.0 & 53.6 & 30.81 & -\end{array}$ 
BARNECAT LIGHTSHIP

1956

September

October

Temperature ${ }^{{ }_{F}}$

Salinity $\%$

Temperature ${ }^{\circ}$

Salinity $\%$

Depth

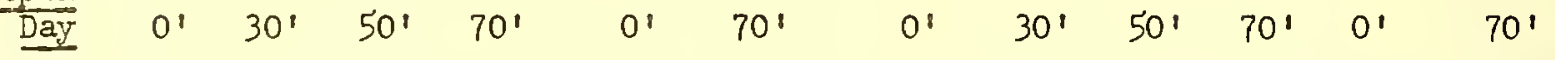

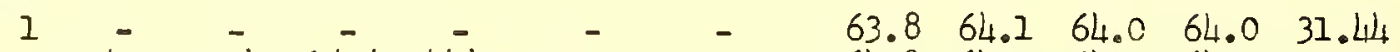

$275.373 .465 .5 \quad 55.4 \quad 30.90 \quad-\quad 64.864 .064 .064 .032 .00$ -

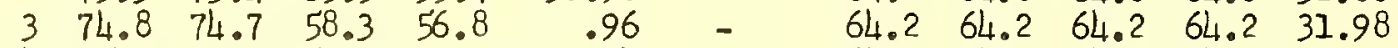

$475.273 .959 .0-94-64.064 .164 .164 .1$ -

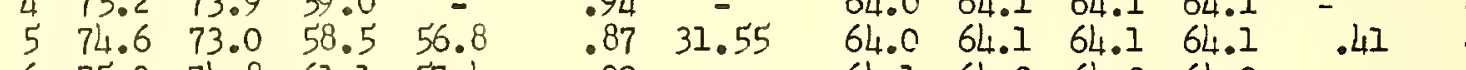

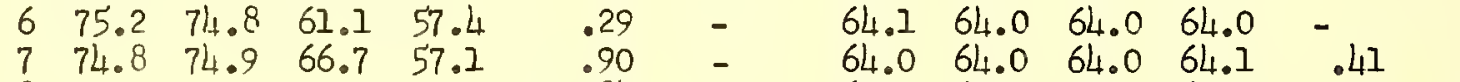

$\begin{array}{ccccccccccccc}7 & 74.8 & 74.9 & 66.7 & 57.1 & .90 & - & 64.0 & 64.0 & 64.0 & 64.1 & .41 & - \\ 8 & - & - & - & - & 30.84 & - & 63.1 & 63.0 & 63.0 & 63.1 & .21 & - \\ 9 & - & - & - & - & - & - & 63.0 & 63.0 & 62.9 & 62.9 & .54 & \end{array}$

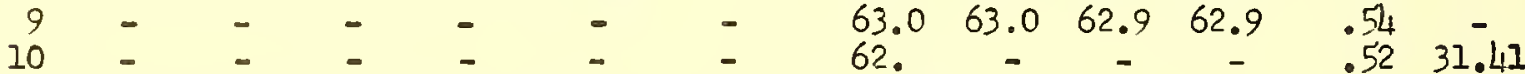

$\begin{array}{llllllllllll}11 & 71.0 & 70.9 & 70.8 & 70.8 & 31.06 & - & 63.0 & 63.0 & - & - & .52\end{array}$

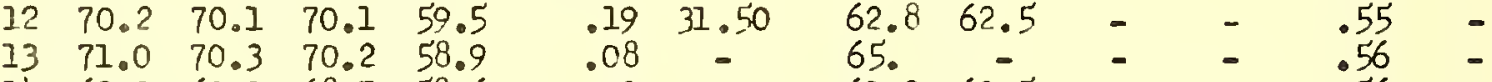

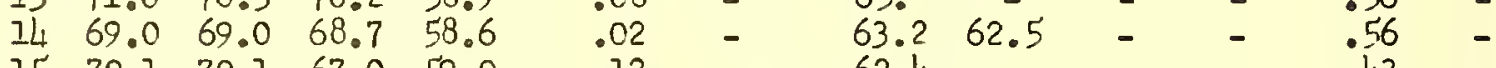

$1570.170 .167 .059 .0 \quad .12-62.4-63-.43-$

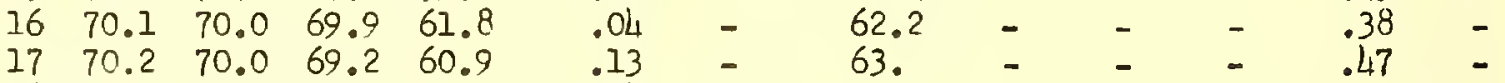

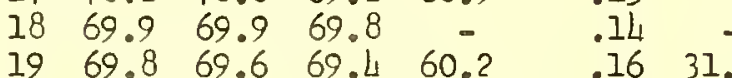

$\begin{array}{llllll}21 & 67.7 & 67.8 & 67.6 & 59.0 & .29\end{array}$

$2266.266 .266 .1 \quad-\quad .18$

$\begin{array}{llllll}23 & - & - & - & - & .34\end{array}$

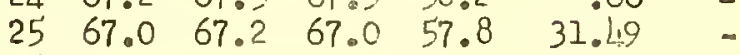

26 - - - -

$\begin{array}{lll}27 & - & -\end{array} 28-$

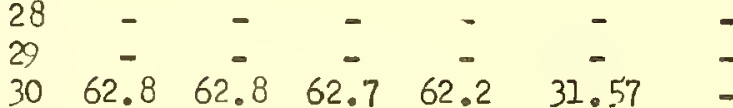

$\begin{array}{ccccccc}30 & 62.8 & 62.8 & 62.7 & 62.2 & 31.57 & -\end{array}$

$\begin{array}{llllll}\text { Mean } \quad 70.5 & 70.2 & 66.4 & 59.4 & 31.11 & 31.46\end{array}$

63. $-\quad-\quad-\quad-\quad-\quad-47=$

63. - - - $-31.77=$

64. - - - $33.25=$

63. - - - 62 - $\quad-.58=$

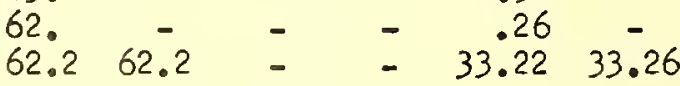

$\begin{array}{ccccc}62.2 & 62.2 & - & 33.22 & 33.26 \\ - & - & - & - & -\end{array}$

$\begin{array}{llllll}- & - & - & - & - & - \\ - & - & - & - & - & -\end{array}$

- - - - - - -

- $\quad$ - $\quad$ - $\quad$ - -100 -

- - - $\quad-31.7832 .70$

- $\quad$ - $\quad-31.9032 .46$ 
BARNEGAT LIGH TSHIP

$$
39^{\circ} 45.8 \mathrm{r} \cdot 75^{\circ} 56.0 \mathrm{r} \cdot
$$

1956

November

$$
\text { Temperature }{ }^{\circ} \mathrm{F}
$$

Depth

Day $0^{\prime} \quad 30^{\prime} 50^{\prime} 70^{\prime} \quad 0^{\prime} 70^{\prime}$

1

2

3

5

$\begin{array}{llll}6 & 58.5 & 58.3\end{array}$

$\begin{array}{lllll}7 & 58.8 & 58.7 & 58.6 & 58.6\end{array}$

$\begin{array}{lllll}8 & 57.9 & 58.7 & 58.7 & 58.7\end{array}$

$\begin{array}{rrrrr}9 & 58.1 & 58.1 & 58.1 & 58.1 \\ 10 & 57.8 & 57.8 & 57.8 & 57.8\end{array}$

$\begin{array}{rrrrrrr}9 & 58.1 & 58.1 & 58.1 & 58.1 & 31.59 & - \\ 10 & 57.8 & 57.8 & 57.8 & 57.8 & .74 & -\end{array}$

$\begin{array}{lllll}11 & 57.1 & 57.1 & 57.1 & 57.1\end{array}$

$\begin{array}{lllll}12 & 57.0 & 56.9 & 56.9 & 56.9\end{array}$

$\begin{array}{lllll}13 & 55.9 & 55.9 & 55.9 & 55.9\end{array}$

$\begin{array}{lllll}14 & 56.2 & 55.7 & 55.7 & 55.7\end{array}$

$\begin{array}{lllll}15 & 55.2 & 55.2 & 55.7 & 56.0\end{array}$

$\begin{array}{lllll}16 & 56.4 & 55.1 & 55.7 & 56.1\end{array}$

$\begin{array}{lllll}17 & 55.1 & 55.2 & 55.2 & 56.7\end{array}$

$\begin{array}{lllll}18 & 55.1 & 55.1 & 55.2 & 56.3\end{array}$

$\begin{array}{lllll}19 & 55.7 & 55.2 & 55.2 & 55.2\end{array}$

$\begin{array}{lllll}20 & 55.1 & 55.1 & 55.2 & 55.2\end{array}$

$\begin{array}{lllll}21 & 54.3 & 54.3 & 55.0 & 55.0\end{array}$

$22 \quad 54.9 \quad 54.9 \quad 54.9 \quad 54.9$

$\begin{array}{lllll}23 & 53.9 & 53.9 & 53.9 & 53.9\end{array}$

$\begin{array}{lllll}24 & 52.9 & 52.9 & 52.9 & 52.9\end{array}$

$\begin{array}{lllll}25 & 52.9 & 52.9 & 52.9 & 52.8\end{array}$

$\begin{array}{lllll}26 & 52.7 & 52.8 & 52.8 & 52.8\end{array}$

$\begin{array}{lllll}27 & 52.4 & 52.4 & 52.4 & 52.4\end{array}$

$\begin{array}{lllll}28 & 51.9 & 51.9 & 51.9 & 51.9\end{array}$

2951

30

31

$.18=$

$.79 \quad-$

$.77=$
Salinity $\%$

$31.65 \quad 31.63$

.68

31.66 -

30.75 -

.81

.7931 .75

$.37 \quad-$

$.45 \quad-$

$.26 \quad-$

$.37 \quad-$

.8131 .79

.81

.50

.56

.48 -

$.48 \quad-$

$\begin{array}{ll}.59 & - \\ .74 & 31.77\end{array}$

$.74 \quad-$

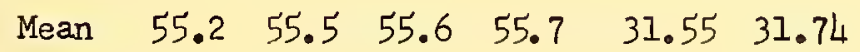


Surface temperatures at Five Fathom Bank Iightship were somewhat lower, month by month, during the first half of the year than the mean for 1947-1955 (Bumpus, 1957), were comparable during the autumn, and significantly warmer at year end. The annual mean was a degree lower than the 1947-1955 mean.

Minimum temperatures of less than $39^{\circ} \mathrm{F}$ occurred surface to bottom during the last third of January and first two-thirds of February and again during the last third of Iarch. A winter maximum of $4 I^{\circ} \mathrm{F}$ was observed during the sècond third of March. The thermocline began to develop in early April. Maximum temperatures of greater than $74^{\circ} \mathrm{F}$ at the surface were observed in mid-August. Maximum temperatures at the bottom were not observed until the fall overturn began during the first third of October. The year ended with the water about $7{ }^{\circ} \mathrm{F}$ warmer throughout the whole column than at the beginning.

As at. Winter Quarter and Chesapeake Iightships the summer warming throughout the water column appears interrupted from time to time. This is most conspicuous during mid-JuIy and late september.

The salinity at the surface commenced between $32.6 \%$ $33 \%$ in January, gradually declined to $30.5 \%$ oo in early June and recovered to $32.5 \%$ at year end. Salinity at the bottom tended to follow the salinity at the surface during the winter and spring months until the thermocline began to develop, following which the bottom salinity tended to keep 10/00 more saline than the surface. 

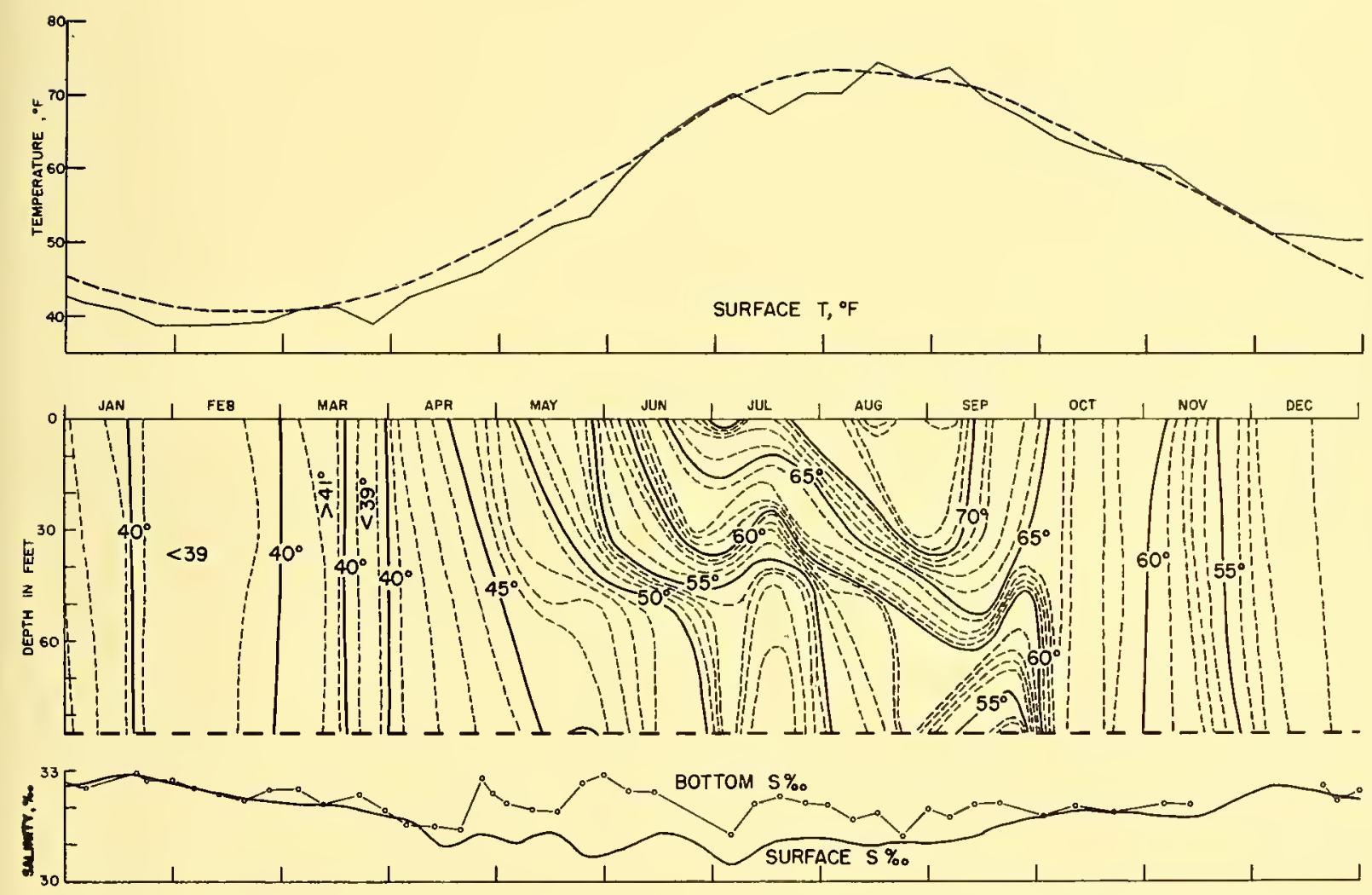

Figure 13. Five Fathom Bank Lightship, 1956. (Dashed Iine in upper diagram, mean for period 1947-1956) 
FIVE FATHOM BANK LIGHTSHIP

$38^{\circ} 47.3 \mathrm{~N} \cdot 74034.6 \mathrm{~W}$ 1955

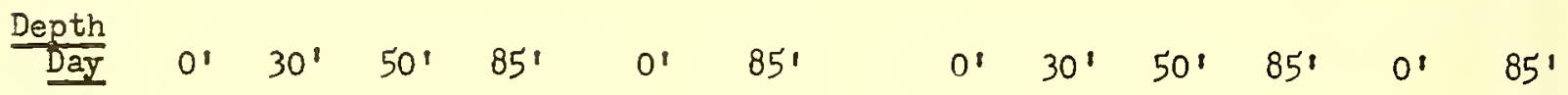

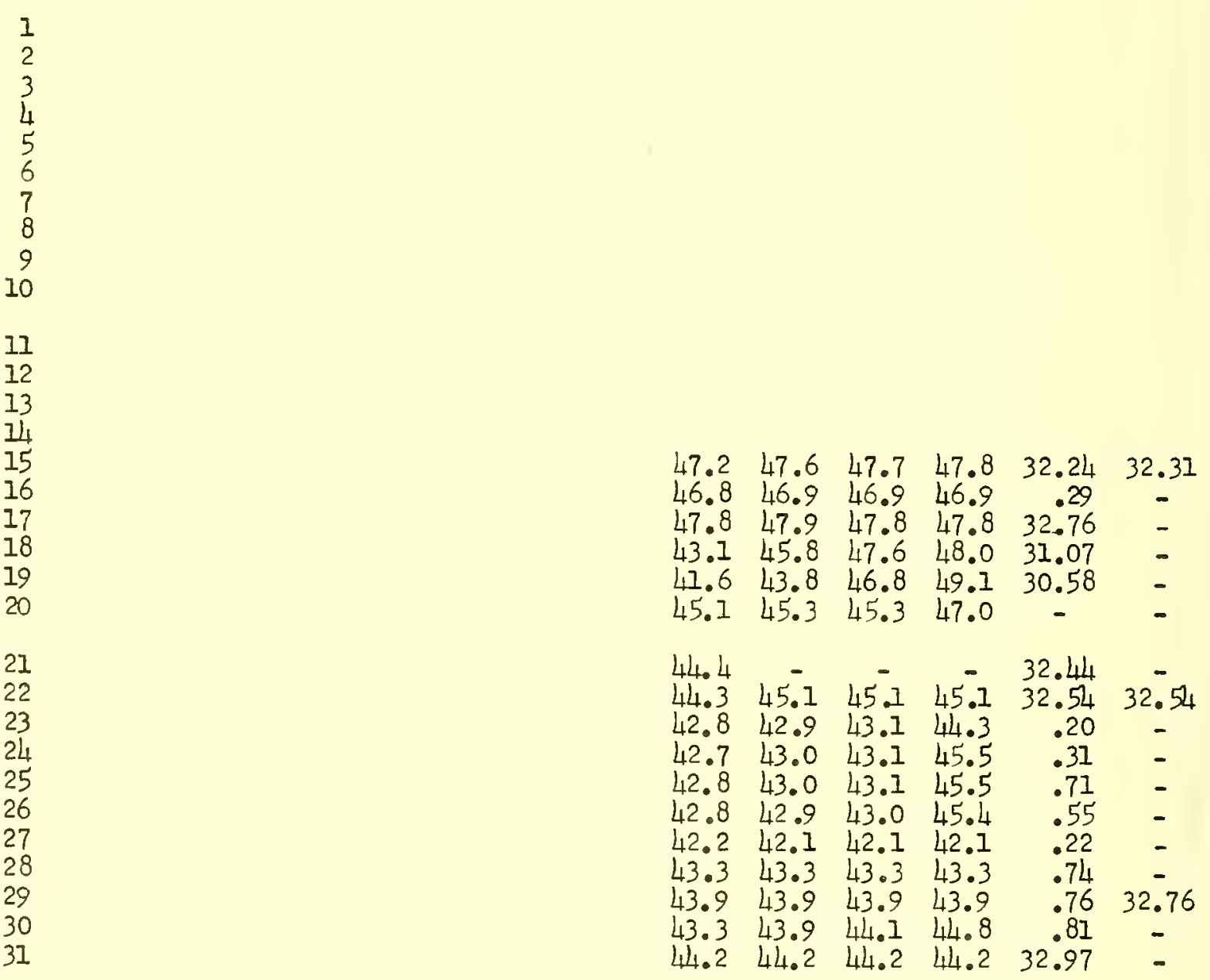


FIVE FATHOM BANK LIGHISHIP

January $38^{\circ} 47.3 \mathrm{~N} \cdot 74^{\circ} 34.6 \mathrm{~W}$. 1956
Salinity $\%$

$0^{\prime} \quad 85^{\prime}$
Da

$\begin{array}{lllll}2 & 41 . & - & - & - \\ 3 & 42.2 & 42.1 & 42.1 & 42.2\end{array}$

$\begin{array}{lllll}4 & 42.6 & 42.7 & 42.8 & 42.8\end{array}$

5

$\begin{array}{lllll}6 & 40.3 & 40.3 & 40.4 & 41.8\end{array}$

$\begin{array}{lllll}7 & 40.1 & 40.1 & 40.2 & 41.8\end{array}$

$$
\begin{aligned}
& 7 \\
& 8
\end{aligned}
$$$$
9
$$

9
10

17

12

13

14

15

16

$\begin{array}{lllll}17 & - & - & - & - \\ 18 & 41.2 & 41.3 & 41.2 & 41.2 \\ 19 & 39.4 & 40.9 & 40.9 & 40.9\end{array}$

$20 \quad 42$

$21 \quad 40.6 \quad 40.3 \quad 40.3 \quad 40.3$

$22 \quad 40.0 \quad 40.0 \quad 40.0 \quad 40.0$

$\begin{array}{llllll}23 & 39.7 & 39.7 & 39.8 & 39.8\end{array}$

$24 \quad 38.6 \quad 38.6 \quad 38.6 \quad 38.6$

$\begin{array}{llllll}25 & 38.9 & 38.9 & 38.9 & 38.9\end{array}$

$\begin{array}{llllll}26 & 38.9 & 38.9 & 38.9 & 38.9\end{array}$

$27 \quad 37.9 \quad 37.9 \quad 38.0 \quad 38.0$

$\begin{array}{lllll}28 & 37.3 & 37.3 & 37.4 & 37.4\end{array}$

$\begin{array}{llllll}29 & 37.6 & 37.6 & 37.6 & 37.6\end{array}$

$\begin{array}{lllll}30 & 38.3 & 38.3 & 38.3 & 38.4\end{array}$

$\begin{array}{llllll}31 & 38.3 & 38.3 & 38.3 & 38.4\end{array}$

\section{$\underline{\text { February }}$}

Salinity $\%$

Depth of water - 87 feet

Salinity $\%$

Temperature ${ }^{\circ} \mathrm{F}$

$0^{\prime} \quad 30^{\prime} \quad 50^{\prime} \quad 85^{\prime} 0^{\prime} 85^{\prime}$

$\begin{array}{llllll}38.5 & 38.5 & 38.6 & 38.6 & 32.74 & -\end{array}$

$\begin{array}{llllll}38.8 & 38.9 & 38.9 & 38.9 & .71 & - \\ 38.0 & 38.0 & 38.0 & 38.5 & .68 & -\end{array}$

$\begin{array}{lllll}39.2 & 38.9 & 38.8 & 38.7 & .67\end{array}$

$\begin{array}{llllll}36.8 & 36.9 & 37.0 & 37.7 & .50 & -\end{array}$

$\begin{array}{llllll}38.7 & 38.7 & 38.7 & 38.8 & .50 & 32.54\end{array}$

$\begin{array}{llllll}39.0 & 39.0 & 39.0 & 39.0 & .52 & -52\end{array}$

$\begin{array}{llllll}40.0 & 39.1 & 39.2 & 39.4 & .56 & -\end{array}$

$\begin{array}{llllll}39.0 & 39.0 & 39.0 & 39.0 & .40 & - \\ 38.6 & 38.9 & 38.9 & 38.9 & .29 & -\end{array}$

$\begin{array}{lllllll}39.1 & 39.1 & 39.1 & 39.1 & .55 & -\end{array}$

$\begin{array}{llllll}38.9 & 38.9 & 38.9 & 38.9 & .34 & - \\ 39.1 & 39.0 & 39.0 & 39.0 & .27 & 32.40\end{array}$

$\begin{array}{llllll}35.0 & 35.0 & 35.0 & 35.0 & .41 & -\end{array}$

$\begin{array}{llllll}39.5 & 39.5 & 39.5 & 39.5 & .35 & -\end{array}$

$\begin{array}{llllll}39.9 & 39.7 & 39.7 & 39.7 & .48 & -\end{array}$

$\begin{array}{llllll}39.3 & 39.5 & 39.5 & 39.5 & .34 & -\end{array}$

40. - - - .26 -

$\begin{array}{llllllll}.92 & 32.92 & 39.1 & 39.1 & 39.1 & 39.2 & .26 & - \\ .98 & 32.98 & 39.4 & 39.1 & 39.1 & 39.1 & .17 & 32.22\end{array}$

$\begin{array}{cccccccc}.82 & - & 39.0 & 39.0 & 39.0 & 39.1 & 32.02 & - \\ .77 & - & 38.7 & 38.7 & 38.7 & 38.8 & 31.96 & - \\ .86 & 32.78 & 38.1 & 38.1 & 38.2 & 38.5 & 32.01 & - \\ .81 & - & 38.1 & 38.0 & 38.0 & 39.0 & 31.89 & - \\ .84 & - & 38.5 & 38.4 & 38.4 & 38.4 & 32.37 & - \\ .82 & - & 39.1 & 39.2 & 39.3 & 39.5 & .36 & - \\ .89 & - & 40.0 & 40.0 & 40.0 & 40.0 & .47 & 32.51 \\ .57 & - & 40.5 & 40.5 & 40.6 & 40.8 & .36 & - \\ .63 & - & 39.6 & 39.5 & 39.5 & 39.5 & .29 & - \\ .75 & 32.76 & & & & & & \end{array}$
32.81 -

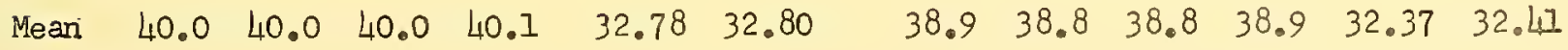


Table 12 (cont'd)

FIVE FATHOM BANK LIGHTSHIP $\quad 38^{\circ} 47.3^{\prime} \mathrm{N} \cdot 74^{\circ} 34.6^{\prime} \mathrm{W}$.

Depth of water - 87 feet 1956

March

April

Temperature ${ }^{\circ} F$

Salinity $\%$ Temperature ${ }^{\circ} \mathrm{F}$

Salinity $\%$

Depth

Day $0^{\prime} \quad 30^{\prime} \quad 5^{\prime} \quad 85^{\prime} \quad 0^{\prime} \quad 85^{\prime} \quad 0^{\prime} \quad 30^{\prime} \quad 50^{\prime} 85^{\prime} \quad 0^{\prime} \quad 85^{\prime}$

$\begin{array}{lllll}5 & 41.3 & 41.2 & 41.1 & 41.1\end{array}$

$\begin{array}{lllll}6 & 41.5 & 47.5 & 47.4 & 41.3 \\ 7 & 41.0 & 40.8 & 40.8 & 40.8\end{array}$

$8 \quad 41.2 \quad 41.2 \quad 41.1 \quad 41 . ?$

$941.0 \quad 41.0 \quad 41.0 \quad 41.1$

$\begin{array}{lllll}10 & 47.1 & 40.7 & 40.7 & 40.7\end{array}$

18

19

20

27 $\begin{array}{lllll}30 & 38.5 & 38.5 & 38.3 & 38.3 \\ 31 & 38.8 & 38.5 & 38.5 & 38.4\end{array}$

$42.0 \quad 42.0 \quad 47.9 \quad 41.9$ $41.0 \quad 41.0 \quad 41.0 \quad 41.0$ $\begin{array}{llll}41.5 & 41.5 & 41.5 & 41.5\end{array}$ $47.5 \quad 47.4 \quad 47.5 \quad 47.4$ $\begin{array}{llll}41.1 & 41.1 & 41.0 & 41.1\end{array}$ $\begin{array}{llll}39.8 & 39.8 & 39.8 & 39.9\end{array}$

$\begin{array}{llll}38.7 & 38.4 & 38.2 & 38.3\end{array}$

$\begin{array}{lllll}48.7 & 38.9 & 39.0 & 39.0\end{array}$

$\begin{array}{lllll}5 & 38.8 & 38.8 & 38.8 & 38.8\end{array}$

$\begin{array}{lllll}26 & 38.5 & 38.6 & 38.6 & 38.6\end{array}$

$\begin{array}{lllll}27 & 39.0 & 38.8 & 38.8 & 38.8\end{array}$

$\begin{array}{lllll}8 & 38.8 & 38.9 & 38.9 & 38.9\end{array}$

$\begin{array}{lllll}38.6 & 38.6 & 38.6 & 38.6\end{array}$ 
FIVE FATHOM BANK LIGHTSHTP

May $38^{\circ} 47.3^{\mathrm{N}} \cdot 74^{\mathrm{O}} 34.6^{\mathrm{W}} \mathrm{W}$. 1956
Salinity $\%$

Day o' $30^{\prime} \quad 50^{\prime} \quad 85^{\prime} \quad 0^{\prime} \quad 85^{\prime}$

$1 \quad 44.0 \quad 43.8 \quad 39.9 \quad 38.6 \quad 31.05$

249 - -30.81

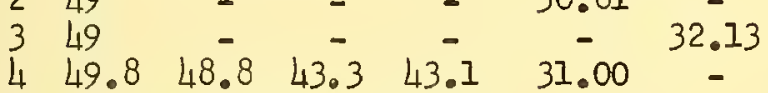

$\begin{array}{lllll}5 & 49.7 & 49.3 & 46.9 & 43.2\end{array}$

$\begin{array}{lllll}6 & 49.7 & 49.1 & 45.0 & 43.2\end{array}$

$\begin{array}{lllll}7 & 50.5 & 49.6 & 46.8 & 43.3\end{array}$

$\begin{array}{lllll}8 & 48.7 & 48.7 & 48.5 & 45.3\end{array}$

$9 \quad 51.0 \quad 48.2 \quad 48.2 \quad 48.0$

$\begin{array}{lllllll}10 & 49.5 & 48.5 & 46.0 & 45.8 & 30.90 & 31.98\end{array}$

$\begin{array}{lllllll}11 & 49.9 & 49.9 & 47.9 & 45.4 & 30.89\end{array}$

$\begin{array}{llllll}12 & 50.6 & 49.8 & 49.0 & 45.3 & 31.15\end{array}$

$\begin{array}{llllll}13 & 51.2 & 45.9 & 45.1 & 45.1 & 31.26\end{array}$

$1454.0 \quad 46.1 \quad 45.0 \quad 44.8$

$\begin{array}{lllll}15 & 52.8 & 46.9 & 46.8 & 46.8\end{array}$

$\begin{array}{lllll}16 & 56.0 & 48.0 & 47.2 & 46.8\end{array}$

$\begin{array}{lllll}17 & 51.5 & 50.6 & 50.2 & 46.4 \\ 18 & 50.4 & 50.2 & 46.8 & 46.0\end{array}$

$\begin{array}{lllll}19 & 51.9 & 50.0 & 47.1 & 45.3\end{array}$

$20 \quad 52.4 \quad 52.0 \quad 45.3 \quad 44.9$

$\begin{array}{lllll}21 & 54.0 & 52.0 & 47.7 & 44.8\end{array}$

$\begin{array}{lllll}22 & 53.2 & 52.9 & 45.0 & 44.8\end{array}$

$\begin{array}{lllll}23 & 53.1 & 45.9 & 44.1 & 43.8\end{array}$

$24 \quad 54.5 \quad 54.0 \quad 51.5 \quad 45.0$

$\begin{array}{lllll}25 & 53.9 & 53.9 & 48.7 & 44.0\end{array}$

$\begin{array}{lllll}26 & 54.7 \quad 54.5 \quad 47.2 \quad 45.0\end{array}$

27 - - - - - -

$\begin{array}{lllllll}28 & 52.8 & 46.3 & 45.0 & 45.0 & 30.97 & -\end{array}$

$\begin{array}{llllll}29 & 52.5 & 52.2 & 46.7 & 45.8 & 30.87\end{array}$

$\begin{array}{llllll}30 & 52.9 & 51.9 & 46.7 & 45.3 & 30.83\end{array}$

$\begin{array}{lllllll}31 & 53.4 & 51.1 & 45.7 & 45.2 & 30.61 & 32.89\end{array}$

31.47

$31.64 \quad 31.90$

31.63 -

$31.60=$

$30.55=$

$30.51=$

$30.91 \quad 32.67$

$-$

$-$

$-$

$-$
June

Temperature ${ }^{\circ} \mathrm{F}$

Salinity $\%$

$0^{\prime} \quad 30^{\prime} \quad 50^{\prime} 85^{\prime} \quad 0^{\prime} \quad 85^{\prime}$

$\begin{array}{lllll}56.8 & 55.1 & 46.2 & 45.5 & 30.81\end{array}$

$\begin{array}{llllll}57.5 & 56.5 & 47.6 & 45.7 & .82 & =\end{array}$

$\begin{array}{lllllll}58.9 & 55.7 & 47.0 & 46.7 & .82 & \text { - }\end{array}$

$\begin{array}{llllll}59.3 & 55.6 & 47.9 & 47.0 & .89 & -\end{array}$

$\begin{array}{llllll}59.9 & 55.1 & 48.8 & 47.0 & 30.86 & -\end{array}$

$\begin{array}{llllll}60.8 & 57.6 & 48.8 & 47.5 & 31.00 & - \\ 60.8 & 57.6 & 48.3 & 48.0 & 30.84 & 32.49\end{array}$

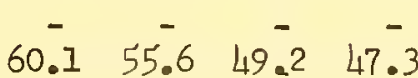

$\begin{array}{lllll}63.7 & 53.5 & 48.2 & 47.8 & 31.00\end{array}$

$\begin{array}{lllll}62.5 & 54.0 & 48.2 & 48.0 & 31.79\end{array}$

$\begin{array}{lllll}62.0 & 54.8 & 48.3 & 48.1 & .22\end{array}$

$63.762 .3-0.26$

$\begin{array}{llllll}64.9 & 54.3 & 49.6 & 49.2 & 31.18 & \end{array}$

$\begin{array}{llllll}64.8 & 56.0 & 49.9 & 49.8 & 31.09 & -\end{array}$

$\begin{array}{lllll}65.5 & 53.4 & 50.7 & 49.4 & .24\end{array}$

$\begin{array}{lllll}64.2 & 63.8 & 51.8 & 50.5 & .46\end{array}$

$\begin{array}{lllll}64.4 & 63.8 & 51.0 & 50.2 & .53\end{array}$

$\begin{array}{llllll}63.9 & 53.8 & 50.0 & 50.0 & .56 & -\end{array}$

$\begin{array}{lllll}65.1 & 64.2 & 50.0 & 49.0 & 31.53\end{array}$

$\begin{array}{lllll}66.9 & 64.1 & 52.5 & 49.5 & .90\end{array}$

$\begin{array}{lllllll}67.6 & 64.9 & 51.7 & 50.3 & .73 & \text { - }\end{array}$

$\begin{array}{llllll}68.5 & 65.6 & 54.4 & 51.0 & 30.82 & -\end{array}$

$\begin{array}{lllll}69.6 & 63.3 & 52.3 & 50.9 & 31.25\end{array}$

$72 \quad 60.0 \quad 57.7 \quad 50.7 \quad 69$

$\begin{array}{lllll}69.0 & 66.2 & 51.7 & 49.5 & 31.36\end{array}$

67.264 .2 5. $649.1 \quad 30.52=$ $\begin{array}{llllll}65.9 & 64.9 & 50.9 & 47.7 & 30.79 & -\end{array}$

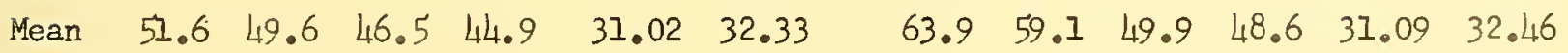


1956

July

Temperature ${ }^{O F}$
Salinity $\%$

$0^{\prime} \quad 85^{\prime}$

\section{August}

Depth

Day

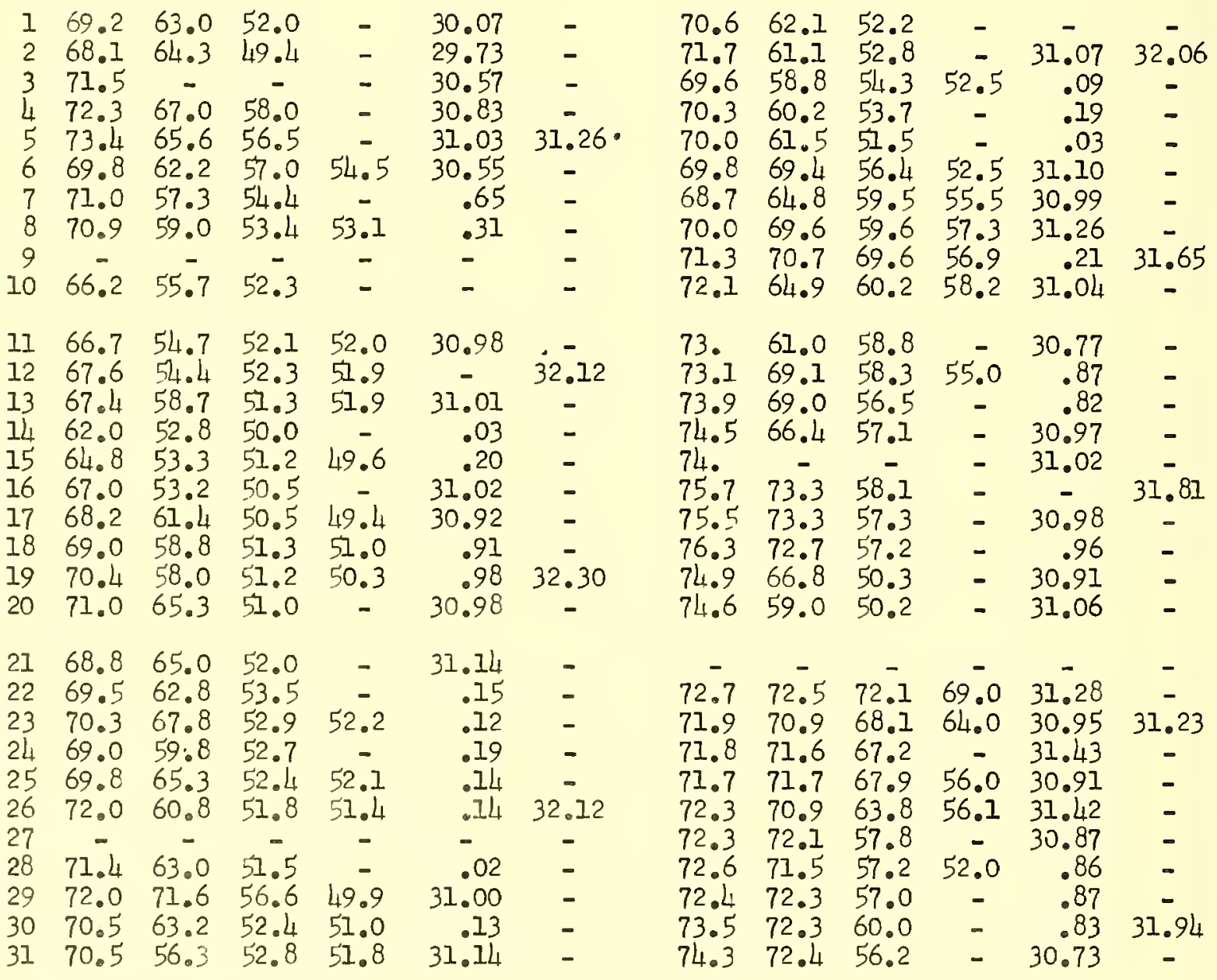

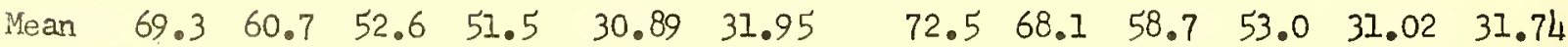


FIVT FA THOM BANK IIGH TSHIP $\quad 38^{\circ} 47.3^{\prime} \mathrm{N} .74^{\circ} 34.6^{\mathrm{w}}$. Depth of water - 87 feet 1956

September

Temperature ${ }^{\mathrm{F}}$ $\begin{array}{lllllll}1 & 74.0 & 73.3 & 59.0 & 51.0 & 30.82 & -\end{array}$

$\begin{array}{lllll}2 & 75.0 & 68.9 & 57.7 & - \\ 3 & 75.0 & 68.3 & 63.2 & 54.0\end{array}$

$\begin{array}{lllll}4 & 74.1 & 72.6 & 62.2 & 55.0\end{array}$

$\begin{array}{lllll}5 & 75.0 & 73.7 & 69.3 & 55.5\end{array}$

$\begin{array}{lllll}6 & 74.5 & 73.7 & 66.0 & 57.0\end{array}$

$\begin{array}{lllll}7 & 75.0 & 74.3 & 65.0 & 55.0\end{array}$

$\begin{array}{lllll}8 & 72.9 & 72.7 & 72.7 & 54.0\end{array}$

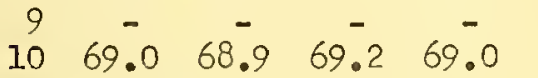

$\begin{array}{llll}11 & 69.0 & 68.6 \quad 68.0\end{array}$

$\begin{array}{lllll}12 & 70.0 & 69.0 & 69.0\end{array}$

$13 \quad 69.5 \quad 67.7 \quad 66.9$

$\begin{array}{llll}14 & 70.0 & 67.7 & 61.6 \\ 15 & 70.0 & 69.4 & 64.3 \\ 16 & 70.5 & 70.4 & 67.4\end{array}$

$16 \quad 70.5 \quad 70.4 \quad 67.4$

$17 \quad 70.5 \quad 69.4 \quad 66.3$

$\begin{array}{lll}18 & 69.3 & 69.3\end{array}$

$\begin{array}{lll}19 & 69.6 & 69.5\end{array}$

$\begin{array}{llll}69.5 & 60.7 & 56.5\end{array}$ 20 22

23

25

26

26
27

27

29

30

31
Salinity $\%$
October

Temperature ${ }^{\mathrm{O}} \mathrm{F}$

Salinity $\% / 00$

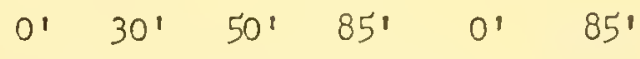

$\begin{array}{rrrrrr}64.5 & 64.9 & 64.8 & 64.8 & 31.73 & - \\ 64.8 & 64.8 & 64.6 & 64.5 & .82 & 31.74\end{array}$

$64.4 \quad 64.2 \quad 64.264 .1 \quad .83 \quad-$

$\begin{array}{cccccccc}30.94 & - & 65.6 & 65.2 & 65.2 & 64.6 & .75 & - \\ 31.16 & - & - & - & - & - & - & -\end{array}$

$\begin{array}{lllllll}.27 & 31.72 & 64.1 & 63.9 & 63.8 & 63.8 & .82\end{array}$

.03 - $64.364 .264 .064 .0 \quad .76$ -

$63.4 \quad 63.4 \quad 63.4 \quad 63.4 \quad .81 \quad-$

$\begin{array}{lllll}63.3 & 63.4 & 63.7 & 63.8 & .89\end{array}$

$63.4 \quad 63.4 \quad 63.2 \quad 63.0 \quad-$

$\begin{array}{lllllll}62.5 & 62.4 & 62.4 & 62.4 & .91 & 32.00\end{array}$

$\begin{array}{llllll}62.6 & 62.1 & 62.1 & 62.1 & .84 & -\end{array}$

$\begin{array}{lllll}62.2 & 62.2 & 62.3 & 62.3 & .90\end{array}$

$\begin{array}{llllll}62.7 & 62.7 & 62.6 & 62.6 & .91 & -\end{array}$

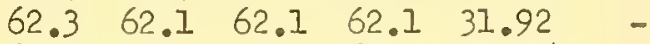

$62.9 \quad 62.1 \quad 62.1 \quad 62.0 \quad 32.15 \quad-$

$\begin{array}{lllll}62.3 & 62.2 & 62.1 & 62.0 & 31.96\end{array}$

$\begin{array}{llllll}62.2 & 62.2 & 62.1 & 62.0 & .97 & -\end{array}$

$\begin{array}{llllll}62.1 & 62.0 & 62.0 & 62.0 & .85\end{array}$

$\begin{array}{cccccc}61.7 & 61.7 & 61.6 & 61.4 & .82 & - \\ - & - & - & - & .87 & - \\ - & - & - & - & - & - \\ - & - & - & - & - & - \\ - & - & - & - & - & - \\ - & - & - & - & - & - \\ 60.5 & 60.3 & 60.3 & 60.2 & .79 & 31.83\end{array}$

$\begin{array}{llllll}63.1 & 63.0 & 62.9 & 62.9 & 31.86 & 31.86\end{array}$ 
Table 12 (cont'd)

FIVE FATHOM BANK LIGHTSHIP $\quad 38^{\circ} 47.3^{\prime} \mathrm{N} \cdot 74^{\circ} 34.6 \mathrm{w}$.

Depth of water - 87 feet 1956

November

December

Temperature ${ }^{\circ} \mathrm{F}$

Salinity $\%$

Temperature ${ }^{\circ} \mathrm{F}$

Salinity $\%$

$\frac{\text { Depth }}{\text { Day }} \quad 0^{\prime} \quad 30^{\prime} 50^{\prime} 85^{\prime} \quad 0^{\prime} 85^{\prime}$

$0^{\prime} \quad 30^{\prime} \quad 50^{\prime} \quad 85^{\prime} \quad 0^{\prime} \quad 85^{\prime}$

$\begin{array}{lllllll}1 & 60.3 & 60.2 & 60.2 & 60.0 & - & 32.09 \\ 2 & 63.9 & 60.5 & 60.2 & 60.1 & 31.72 & \end{array}$

-
-
-
-
.06

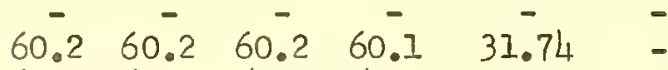

$\begin{array}{lllll}60.1 & 60.1 & 59.9 & 59.9 & .70\end{array}$

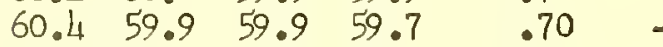

$\begin{array}{rrrrrr}59.2 & 58.9 & 58.9 & 58.8 & .71 & - \\ 59.8 & 59.1 & 59.0 & 59.0 & 31.70 & 32.06\end{array}$

- - - -

$-$

$-$

11

$\begin{array}{lllll}58.7 & 57.9 & 57.9 & 58.1 & 32.12\end{array}$

$-$

13

14

15

19

$\begin{array}{ccccc}58.2 & 57.9 & 57.9 & 58.1 & 32.12 \\ - & - & - & - & -\end{array}$

$\begin{array}{lllll}- & - & - & - & - \\ - & - & - & - & -\end{array}$

$\begin{array}{lllll}57.3 & 56.6 & 57.9 & 58.6 & 31.54\end{array}$

$\begin{array}{llllllll}56.7 & 57.1 & 57.1 & 59.8 & .54\end{array}$

$\begin{array}{llllll}56.0 & 56.0 & 56.1 & 56.0 & - & .04\end{array}$

$\begin{array}{llllll}21 & 56.1 & 56.2 & 56.5 & 56.5 & 31.74\end{array}$

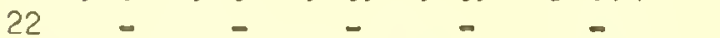

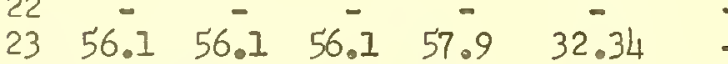

$\begin{array}{llllll}24 & 54.4 & 54.6 & 54.7 & 55.7 & .16\end{array}$

$25 \quad 54.6 \quad 54.1 \quad 54.3 \quad 54.5 \quad 31.95 \quad$

$\begin{array}{llllll}26 & 53.2 & 53.9 & 54.3 & 54.7 & 32.00\end{array}$

$\begin{array}{llllll}27 & 52.8 & 53.2 & 53.2 & 54.6 & .10\end{array}$

28 53. - - - .16 -

$\begin{array}{lllllll}29 & 50.7 & 52.8 & 52.8 & 53.0 & 32.38 & -\end{array}$

31

$\begin{array}{llllll}\text { Mean } & 57.1 \quad 57.1 \quad 57.2 \quad 57.6 \quad 31.89(32.08)\end{array}$

$\begin{array}{lllll}52.2 & 52.2 & 52.2 & 52.2 & 32.49\end{array}$

$\begin{array}{llll}52.3 & 52.3 & 52.3 & 52.9\end{array}$

$\begin{array}{lllll}52.3 & 52.3 & 52.5 & -\end{array}$

$50.8 \quad 52.8 \quad 53.2 \quad 53.6$

$51.551 .4 \quad 51.15 \overline{1} 5$

$50.2 \quad 50.6 \quad 51.7 \quad 51.9$

$51.5 \quad 51.6 \quad 52.0 \quad 52.9$

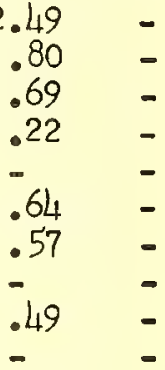

$51.0 \quad 51.0 \quad 51.951 .9$

$52.3 \quad 52.3 \quad 52.6 \quad 52.9$

$\begin{array}{llll}52.8 & 52.9 \quad 53.6 \quad 53.6\end{array}$

.66

$.84 \quad-$

.17

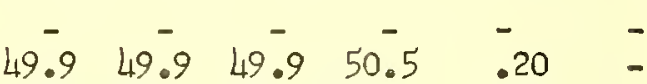

$\begin{array}{llllll}50.4 & 50.2 & 50.2 & 51.1 & .36 & -\end{array}$

$\begin{array}{llllll}50.8 & 50.8 & 50.9 & 51.1 & .38 & -\end{array}$

$\begin{array}{llll}50.9 & 50.9 & 50.9 & 50.7\end{array}$

.69

. 32.59

$51.051 .051 .551 .9 \quad .56$ -

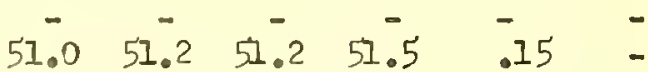

$51.351 .351 .351 .3=32.17$

$51.151 .0 \quad 50.951 .2 \quad .46 \quad$ -

$51.351 .351 .451 .6 \quad .34$ -

$\begin{array}{llllll}50.7 & 50.7 & 50.6 & 50.7 & .13 & -\end{array}$

$\begin{array}{llllll}49.9 & 49.8 & 49.8 & 49.8 & - & - \\ 49.7 & 49.7 & 49.7 & 49.7 & .17 & -\end{array}$

$\begin{array}{llllll}49.8 & 49.8 & 49.7 & 49.8 & .31 & =\end{array}$

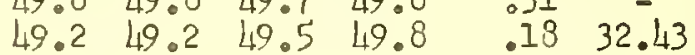

$51.0 \quad 51.1 \quad 51.3 \quad 51.5 \quad 32.44(32.40)$ 
Monthly mean surface temperatures exhibited no significant departure from the mean for the periods $1879-99$ and $1925-40$ (Bumpus, 1957) although the amual mean for $1956\left(56.3^{\circ} \mathrm{F}\right)$ was higher than the period of record amual means (1879-99; 55.4\% F; $\left.1925-40,55.9^{\circ} \mathrm{F}\right)$.

Minimum temperatures throughout the water column occurred during the latter part of January and first-third of February at approximately $40^{\circ} \mathrm{F}$. The thermocline commenced development in April. Maximum temperatures at the surface $\left(76^{\circ} \mathrm{F}\right)$ were reached during the last third of August. Maximum temperatures may have been missed at the bottom during September. By the first-third of October the autumn overturn had commenced whereupon the temperature declined at a regular rate throushout the water column. The year ended with the temperatures about $7^{\circ} \mathrm{F}$ warmer than at the end of 1955. The intrusion of off-shore water during mid-July chilled the whole column of water several degrees. This is apparent in the temperature time profile, as it was also at Five Fathom Lightship.

Surface salinities remained near $32.5 \%$ during the first $22 / 3$ months, dropped to $31 \%$ in early April, recovered to $32 \%$ during May, gradually declined to $31 \%$ in August and slowly recovered to $32.5 \%$ at year end. Bottom salinities tended to remain fairly close to the surface salinities during the first quarter of the year, became higher by 1 to $1 \frac{1}{2}$ parts per thousand while the thermocline was developed then returned to only slightly higher than surface values during the last quarter of the year. 

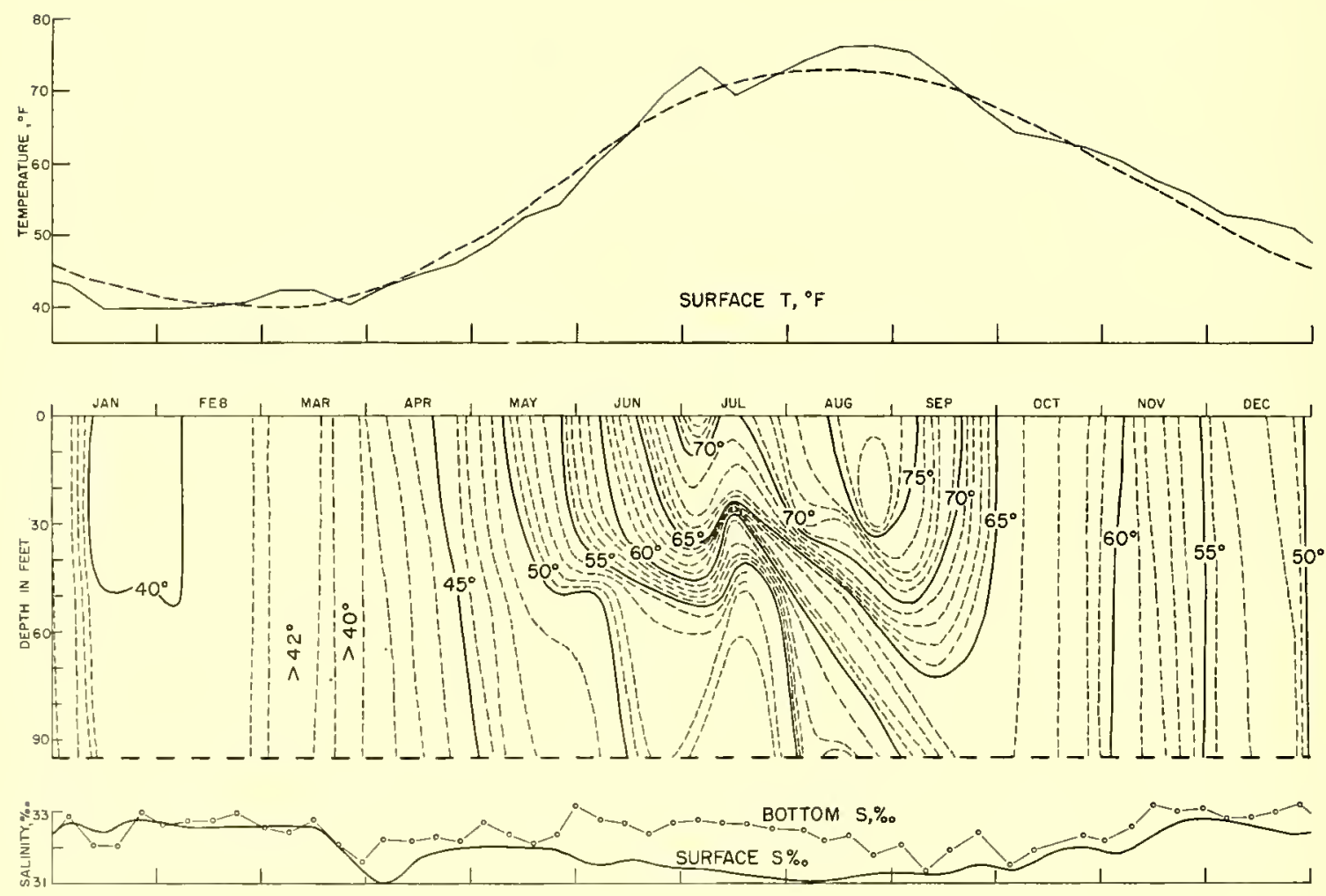

Figure 14. Winter Quarter Lightship, 1956. (Dashed line in unper diagran, mean for period 1925-19L.0) 


\section{December}

Temperature ${ }^{\circ}$ F Salinity $\%$

$\frac{\text { Depth }}{\text { Day }}$

$0^{\prime} 30^{\prime} 50^{\prime} \quad 96^{\prime} \quad 0^{\prime} \quad 96^{\prime}$

$\begin{array}{llllll}53.6 & 53.6 & 53.6 & 53.6 & 32.58 & 32.41\end{array}$ $\begin{array}{llllll}53.0 & 53.0 & 53.0 & 53.0 & .26 & -\end{array}$ $\begin{array}{llllll}52.5 & 52.5 & 52.9 & 52.9 & .28 & -\end{array}$ $\begin{array}{llllll}52.4 & 52.6 & 52.8 & 52.8 & .22 & -\end{array}$ $\begin{array}{llllll}52.1 & 52.1 & 52.1 & 52.6 & .10 & -\end{array}$ $\begin{array}{llllll}52.2 & 52.3 & 52.3 & 52.3 & .30 & -\end{array}$

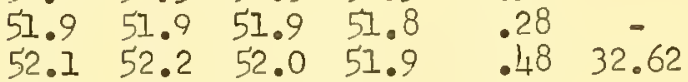
$51.251 .2 \quad 51.2 \quad 51.1 \quad .28$ $\begin{array}{lllll}50.3 & 50.3 & 50.3 & 50.3 & .16\end{array}$

11

12

13

14

15

16

17

18

19

20

21

22

23

24

25

26

27

28

29

30

31

Mean $\begin{array}{lllllll}49.2 & 49.4 & 49.2 & 49.3 \quad .90 & -\end{array}$

$\begin{array}{llllll}48.8 & 49.3 & 49.6 & 50.5 & .20 & -\end{array}$

$\begin{array}{llllll}48.7 & 48.8 & 49.0 & 50.6 & 32.14 & -\end{array}$

$\begin{array}{lllll}46.4 & 46.9 & 46.8 & 48.2 & 31.42\end{array}$

$\begin{array}{lllll}48.4 & 48.7 & 49.0 & 49.0 & 32.12\end{array}$

$\begin{array}{lllll}48.5 & 48.9 & 48.7 & 48.5 & 32.32\end{array}$

$\begin{array}{lllll}46.0 & 47.3 & 48.2 & 48.8 & 31.82\end{array}$

$\begin{array}{lllll}47.8 & 47.9 & 48.4 & 49.0 & 32.39\end{array}$

$\begin{array}{lllll}47.2 & 47.2 & 48.5 & 49.1 & .30\end{array}$

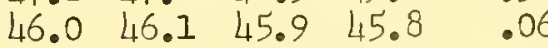

$\begin{array}{llllll}45.1 & 45.1 & 45.1 & 45.0 \quad 04\end{array}$

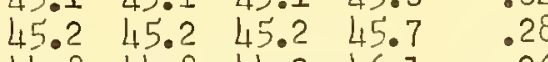

$\begin{array}{lllll}44.8 & 44.8 & 44.9 & 46.1 & .26\end{array}$

$44.844 .844 .9 \quad-\quad .22$

45.5 - $-\quad$ - .16

$\begin{array}{lllll}44.6 & 44.6 & 44.6 & 46.7 & .12\end{array}$

$44.3 \quad 44.4 \quad 44.4 \quad 44.5 \quad 32.22 \quad-$

$\begin{array}{llllll}42.7 & 42.7 & 42.7 & 42.7 & 31.72 & -\end{array}$

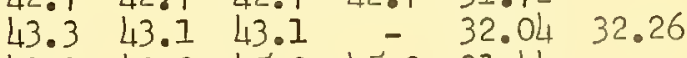

$\begin{array}{llllll}41.1 & 43.2 & 45.0 & 45.0 & 31.44 & - \\ 44.2 & 44.3 & 44.8 & 44.9 & 32.50 & -\end{array}$

$\begin{array}{llllll}47.9 & 48.1 & 48.3 & 49.0 & 32.18 & 32.40\end{array}$ 


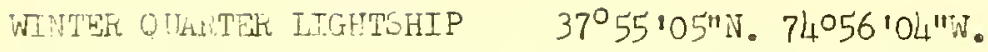

Deptri of water - 96 feet

1956

January

Temperature ${ }_{F}$ Salinity $\%$ Temperature $O_{T}$

February

Denth

$0^{\prime} \quad 30^{\prime} \quad 50^{\prime} \quad 96^{\prime} \quad 0^{\prime} \quad 96^{\prime}$

$0^{\prime} \quad 30^{\prime} \quad 50^{\prime} \quad 96^{\prime} \quad 0^{\prime} \quad 96^{\prime}$

$1 \quad 41.1 \quad 42.0 \quad 44.2 \quad 45.2 \quad 31.50 \quad-$

$\begin{array}{lllll}43.8 & 43.8 & 43.8 & 43.8 & 32.46=\end{array}$

$43.1 \quad 43.0 \quad 43.0 \quad 43.0$

$\begin{array}{lllll}43.1 & 43.1 & 43.4 & 43.1\end{array}$

$\begin{array}{lllll}43.3 \quad 43.2 & 43.1 & 43.1\end{array}$

$\begin{array}{lllll}6 & 44.7 & 44.8 & 44.7 & 44.7\end{array}$

$\begin{array}{lllll}7 & 44.9 & 44.9 & 44.9 & 44.9\end{array}$

$\begin{array}{lllll}8 & 42.3 & 42.4 & 42.4 & 42.4\end{array}$

$9 \quad 43.1 \quad 43.0 \quad 43.1 \quad 43.1$

$\begin{array}{lllll}10 & 42.3 & 42.3 & 42.2 & 42.2\end{array}$

.45

$32.86 \quad 32.87^{\circ}$

$\begin{array}{llll}39.2 & 39.3 & 39.6 \quad 40.2\end{array}$

$\begin{array}{llll}39.7 & 39.4 & 39.3 & 37.3\end{array}$

$\begin{array}{lllll}30.8 & 39.6 & 39.8 & 40.1\end{array}$

$33.14 \quad-$

$\begin{array}{llll}39.8 & 39.7 & 39.8 & 39.8\end{array}$

.15

$.07 \quad-$

$33.06=$

$\begin{array}{llll}39.6 & 39.5 & 39.7 & 39.7\end{array}$

$\begin{array}{llll}39.9 & 39.7 & 39.7 & 39.7\end{array}$

$\begin{array}{llll}39.5 & 39.5 & 39.5 \quad 40.0\end{array}$

$\begin{array}{llll}40.0 & 39.8 & 40.3 \quad 40.5\end{array}$

$\begin{array}{lllll}39.9 & 39.7 & 40.3 & 40.3\end{array}$

$32.87-$

$40.4 \quad 40.1 \quad 40.0 \quad 40.0$

32.67

$.65 \quad 32.65$

$1147.5 \quad 41.3 \quad 41.3 \quad 41.3$

$12 \quad 40.0 \quad 40.0 \quad 40.0 \quad 40.0$

$.48 \%$

$13 \quad 38.6 \quad 38.4 \quad 38.5 \quad 38.5$

$32.08 \quad 32.0$

31.9].

$\begin{array}{llll}39.7 & 39.7 & 39.7 & 39.5\end{array}$

$\begin{array}{lllll}39.9 & 39.8 & 39.8 & 39.8\end{array}$

$\begin{array}{llll}39.5 & 40.0 \quad 40.0 \quad 40.0\end{array}$

$\begin{array}{llll}39.2 & 39.7 & 39.7 & 39.9\end{array}$

$\begin{array}{llll}39.1 & 39.4 & 39.9 & 40.2\end{array}$

$\begin{array}{lllll}15 & 39.0 & 39.1 & 39.3 & 39.7\end{array}$

$32.34=$

$-$

.31

40.0

39.

40.3

$40.2 \quad 40.0$

$40.7-40.5$ L0.5 40.0

Salinity $\% / 00$

$\begin{array}{lllll}17 & 30.9 & 39.0 & 39.0 & 40.9\end{array}$

$\begin{array}{lllll}18 & 39.9 & 40.0 & 40.0 & 40.0\end{array}$

$2047.4 \quad 47.3 \quad 47.3 \quad 47.3$

$32.86=$

$33.05 \quad 32.04$

$214 I .2 \quad 41.1 \quad 41.1 \quad 1.1$

$\begin{array}{lllll}22 & 41.0 & 40.9 & 40.9 & 40.9\end{array}$

$23 \quad 39.2 \quad 40.8 \quad 41.0 \quad 41.1$

$\begin{array}{lllll}24 & 39.9 & 39.9 & 39.9 & 39.9 \\ 25 & 39.8 & 39.8 & 39.9 & 40.9\end{array}$

$\begin{array}{ccccccc}26 & 39.8 & 39.9 & 39 . ? & - & 32.75 & 32.4\end{array}$

$.08=$

41.3 LI.2 4I.I 41.0

.60

.59

.68

.52

.57

$.43 \quad 32.72$

$2840.1 \quad 40.0 \quad 40.1 \quad 40.1 \quad 33.0 ?=$

$\begin{array}{lllllll}29 & 38.8 & 38.6 & 39.8 & 40.6 & 32.56 & -\end{array}$

$\begin{array}{llllll}30 & 38.7 & 38.6 & 40.2 & 40.5 & .66\end{array}=$

$41.0 \quad 40.9 \quad 40.9 \quad 40.9$

$\begin{array}{llll}40.0 & 40.0 & 0.2 \quad 40.2\end{array}$

$\begin{array}{llll}39.4 & 39.5 & 40.3 & 40.8\end{array}$

$39.2 \quad 39.2 \quad 39.4 \quad 40.3$

$40.2 \quad 40.1 \quad 40.0 \quad 40.0$

.55

$\begin{array}{lllll}40.5 & 40.5 & 40.2 \quad 40.2 \quad .45 & -4\end{array}$

$41.541 .7 \quad 41.7 \quad 41.6 \quad .70=$

$\begin{array}{llllll}42.8 & 42.9 & 42.7 & 42.7 \quad 0.85 & -\end{array}$

$\begin{array}{llllll}31 & 39.0 & 39.0 & 39.1 & 39.7 & 32.69\end{array}$

$\begin{array}{lllllllllllll}\text { Mean } & 40.9 & 41.0 & 41.2 & 41.5 & 32.66 & 32.50 & 40.1 & 40.1 & 40.2 & 40.3 & 32.56 & 32.78\end{array}$ 
1956

Temperature ${ }^{\circ} \stackrel{\text { March }}{\text { Salinity } \% 00}$ Temperature ${ }_{T}^{\text {April }}$ Salinity $\% / 00$

$\frac{\text { Depth }}{\text { Day }} \quad 0^{\prime} \quad 30^{\prime} \quad 50^{\prime} 96^{\prime} \quad 0^{\prime} \quad 96^{\prime}$

$0^{\prime} 30^{\prime} 50^{\prime} 96^{\prime} \quad 0^{\prime} 96^{\prime}$

$\begin{array}{lllll}1 & 40.7 & 40.4 & 40.4 & 40.2\end{array}$

$\begin{array}{llll}2 & 41.5 & 41.5 \quad 41.5 & 41.5\end{array}$

$32.47 \quad 32.54$

$\begin{array}{lllll}3 & 42.0 & 42.0 & 42.4 & 42.4\end{array}$

$\begin{array}{ll}.65 & - \\ .72 & = \\ .76 & - \\ .73 & = \\ .62 & = \\ .41 & - \\ .42 & 32.42 \\ .42 & - \\ .75 & -\end{array}$

$41.0 \quad 40.2$

$41.7 \quad 41.1$

42.

41.7

40.1

$40.4 \quad 30.95$

42.

40.340 .5

43.

41.6

$40.9 \quad 40.8$

$\begin{array}{llllll}5 & 42.5 & 42.5 & 42.5 & 42.6\end{array}$

44.2

42.8

$43.9 \quad 42.7$

41.

$43.2 \quad 43.1$

41.2

$\begin{array}{llllll}7 & 42.5 & 42.2 & 42.1 & 42.1\end{array}$

$842.4 \quad 42.4 \quad 42 \% 4 \quad 42.0$

$\begin{array}{lllll}9 & 42.3 & 42.2 & 42.2 & 42.2\end{array}$

$10 \quad 44.2 \quad 43.8 \quad 44.0 \quad 44.0$

$42.7 \quad 42.3$

47.3

43.0

.99

.77

.90

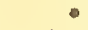

$\begin{array}{llllll}11 & 44.0 & 43.9 & 43.8 & 43.8 & .82\end{array}$

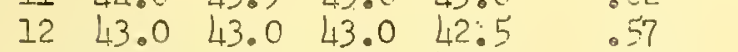

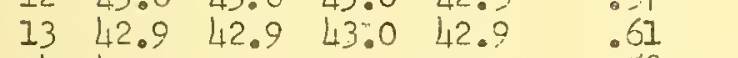

$1443.0 \quad-\quad-\quad-\quad .58$

$\begin{array}{lllll}15 & 42.7 & 42.7 & 42.7 & 42.5\end{array}$

16

$\begin{array}{lllll}17 & 42.1 & 42.0 & 42.0 & 42 .\end{array}$

.58

32.7

$-$

$42.6 \quad 42$.

$42.7 \quad 42.7$

$.47 \quad 32.21$

.8

.82

.35

.56

$19 \quad 41.2 \quad 41.0 \quad 47.0 \quad 41.0$

.25

42.9

42,4

42.

$43.7 \quad 42.7$

42.

$12^{-} 2$

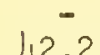

31.54

$-$

42.6

45.1

43.2

$44.4 \quad 43.7$

$42.3 \quad 42.1$

32.22

$47.1 \quad 44.9 \quad 43.9$

44.15

42.8

.10

.17

0.71

31.85

.47

$43.5 \quad 31.42$

$45.9 \quad 45.4 \quad 44.9$

44.

$45.6 \quad 44.9$

44.0

43.9

.05

$20 \quad 40.1 \quad 40.2,40.1 \quad 40.0$

$21 \quad 40.0 \quad 40.0 \quad 40.0 \quad 39.9$

17

\section{2}

23

(2)

$24 \quad 40.0 \quad L O . O \quad L O .0 \quad L O: 0$

$\therefore .00 \quad 32.05$

$\begin{array}{llll}45.1 & 45.0 & 44.7 \quad 43.9\end{array}$

$.14 \quad-$.

$\begin{array}{llllll}25 & 40.2 & 40.2 & 40.7 & 40.0\end{array}$

$\begin{array}{lllll}26 & 40.0 & 39.8 & 39.9 & 40.0\end{array}$

$\begin{array}{lllll}27 & 41.7 & 40.8 & 40.5 & 40.4 \\ 28 & 41.2 & 41.0 & 41.0 & 40.3\end{array}$

$29 \quad 40.2140 .0 \quad 40.0 \quad 40.4$

$\begin{array}{lllll}30 & L 0.3 \quad 40.0 \quad L 0.0 & 39.9\end{array}$

31.95

32.08

31.66

$3 . .1 .6$

$31 . ? 8$

$3 . \div 2$

$31.47 \quad 31.60$

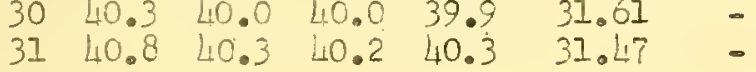

$45.7 \quad 44.9 \quad 44.7 \quad 44.7$

$45.7 \quad 44.6 \quad 44.1 \quad 43.9$

$45.2 \quad 45.3 \quad 44.6 \quad 44.3$

.72

$45.0 \quad 44.9 \quad 44.9 \quad 44.8 \quad 32.11$

$\begin{array}{lllll}45.1 & 44.9 & 44.9 & 44.8 \quad .70\end{array}$

$\begin{array}{llllll}45.6 & 45.1 & 45.0 & 44.9 & 32.20 & 32.18\end{array}$

$\begin{array}{llllll}46.2 & 45.8 & 45.1 & 44.8 & 31.73 & -\end{array}$

$\begin{array}{lllll}47.7 & 46.3 & 44.0 & 43.9 & 31.83\end{array}$

$47.4 \quad 45.9 \quad 44.1 \quad 44.1 \quad 32.22$

32.16

Mean 42.7 LI.5 41.5 L1.4 $32.31 \quad 32.27$

$44.4 \quad 43.7 \quad 43.1 \quad 43.0 \quad 31.55 \quad 32.21$ 
$37^{\circ} 55^{\prime} 05^{\prime \prime N} .74^{\circ} 56^{\prime} 04^{\prime \prime w}$. 1956
Depth of water - 96 feet May 75 feet June
Temperature ${ }^{o_{F}} \stackrel{\text { May }}{ }$

$\frac{\text { Depth }}{\text { Day }} \quad 0^{\prime} \quad 30^{\prime} 50^{\prime} \quad 96^{\prime} \quad 0^{\prime} 96^{\prime}$

$\begin{array}{lllll}1 & 47.8 & 47.7 & 45.6 & 44.4\end{array}$

$248.2 \quad 47.9 \quad 46.2 \quad 44.7$

$\begin{array}{ccccc}3 & 48.7 & 47.6 & 45.9 & 44.9 \\ 4 & 49 . & - & 46.9 & 44.9\end{array}$

$\begin{array}{lllll}5 & 48.4 & 47.9 & 46.4 & 45.3\end{array}$

$\begin{array}{lllll}6 & 49.0 & 48.5 & 47.5 & 45.1\end{array}$

$\begin{array}{llllll}7 & 49.5 & 48.2 \quad- & -\end{array}$

$8 \quad 47.8 \quad 47.8 \quad 47.8 \quad-$

$\begin{array}{lllll}9 & 49.2 & 48.2 & 47.9 & 47.1\end{array}$

$10 \quad 48.4 \quad 48.4 \quad 47.8 \quad 47.5$

11

12

\section{1 \\ 1}

\section{1}

16

22

\section{3}

\section{4}

\section{$49.249 .2 \quad 48$.}

50.8 4..

$\begin{array}{lll}49.4 & 48.2\end{array}$

$\begin{array}{llll}50.2 & 48.2 & 48.1 & 48.1\end{array}$

$\begin{array}{lllll}53.4 & 49.1 & 48.5 \quad 48.0\end{array}$

$52.5 \quad 51.1 \quad 48.4 \quad 48.5$

$\begin{array}{lllll}54.5 & 49.0 & 48.7 & 48.5\end{array}$

$\begin{array}{lllll}53.0 & 50.2 & 49.5 \quad 48.9\end{array}$

$\begin{array}{llll}53.5 & 52.7 & 51.9 & 49.0\end{array}$

$54.7 \quad 5.0 \quad 19.0 \quad 47.3$

$\begin{array}{llll}52.5 & 52.1 & 50.8 & 46.6\end{array}$

$\begin{array}{lllll}52.7 & 52.0 & 50.7 & 47.6\end{array}$

$54.0 \quad 52.0 \quad 51.9 \quad 47.5$

$\begin{array}{llll}54.5 & 53.7 & 47.5 & 47.1\end{array}$

$53.5 \quad 53.2 \quad 52.3 \quad 48.1$

$54.0 \quad 53.5 \quad 5.0 \quad 48.8$

$\begin{array}{lllll}54.2 & 53.7 & 48.7 & 48.3\end{array}$

$54.2 \quad 54.0 \quad 47.6 \quad 47.1$

54.0 5. $148.0 \quad 48.1$

$\begin{array}{lllll}54.3 & 53.9 & 52.9 & 49.2\end{array}$

$54.3 \quad 54.0 \quad 52.1 \quad 48.5$

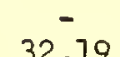

32.19

$32.16 \quad 32.72$

32.08

31.90

32.03

31.96

31.74

32.38

$.01 \quad 32.37$

$.40 \quad-$

32.23

31.80

32.25

31.85

32.16

$31.54 \quad 32.12$

31.64

32.33

32.40

31.7

.80

.84

$$
.74
$$

.77

31.84

32.20

31.92

32.12

31.83

-

-

$-$

$-$

-

$-$

-

-

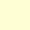

$-$

$-$

$-$

$-$

$-$

$-$

32.35

$-$

$-$

$-$

-

$-$

33.16

\author{
June \\ Temperature ${ }^{\circ}$ F Salinity $\%$
} $0^{\prime} 30^{\prime} 50^{\prime} 75^{\circ} \quad 0^{\prime} \quad 75^{\circ}$

$\begin{array}{lllll}57.9 & 55.8 & 48.5 & 47.9\end{array}$

$\begin{array}{llllll}58.6 & 57.9 & 50.7 & 48.7 & 30.29\end{array}$

$\begin{array}{llllll}58.5 & 56.0 & 49.4 & 48.1 & 31.50\end{array}$

$60.3 \quad 57.2 \quad 49.7 \quad 47.8$

$60.1 \quad 57.2 \quad 49.7 \quad 48.1$

$61.0 \quad 58.3 \quad 5.0 \quad 48.4$

$57.7 \quad 54.6 \quad 46.2 \quad 45.4$

$.56=$

- $\quad$ -

$\begin{array}{ll}.83 & 32.77\end{array}$

$\begin{array}{lllll}-51.5 & 61 . \overline{4} & 49.4 & 49.1 & 31.84\end{array}$

$\begin{array}{lllll}61.8 & 59.8 & 50.9 & 50.6 & 32.09\end{array}$

$\begin{array}{lllll}62.3 & 61.6 & 54.9 & 50.2 & .01\end{array}$

$61.5 \quad 60.6 \quad 51.0 \quad 49.0 \quad 32.00$

$\begin{array}{llllll}64.5 & 61.3 & 51.7 & 49.8 & 31.83\end{array}$

$\begin{array}{llllll}68.0 & 62.3 & 53.8 & 50.2 & 30.72 & 32.66\end{array}$

$\begin{array}{llllll}63.8 & 52.6 & 48.8 & 48.2 & 30.88 & -\end{array}$

$\begin{array}{cccccc}- & - & - & - & - & - \\ 64.1 & 64.1 & 54.9 & 54.5 & 32.19 & -\end{array}$

$\begin{array}{lllll}63.9 & 63.5 & 55.7 & 54.2 & 32.10\end{array}$

$\begin{array}{llllll}64.5 & 64.2 & 54.4 & 52.5 & 31.98 & 32.39\end{array}$

$\begin{array}{llllll}66.3 & 65.7 & 52.8 & 52.0 & .28 & -\end{array}$

$\begin{array}{llllll}68.7 & 65.6 & 53.0 & 52.1 & .22 & -\end{array}$

$\begin{array}{llllll}68.0 & 65.6 & 57.0 & 52.9 & .26 & -\end{array}$

$\begin{array}{lllll}71.0 & 61.0 & 53.0 & 52.6 & .36\end{array}$

$\begin{array}{llllll}71.3 & 61.0 & 54.0 & 52.5 & .63 & -\end{array}$

$\begin{array}{lllll}71.5 & 62.6 & 52.9 & 51.3 & .37\end{array}$

$\begin{array}{llllll}73.5 & 65.3 & 55.0 & 51.9 & .28 & 32.70\end{array}$

$\begin{array}{llll}70.6 & 63.2 & 55.5 & 52.0\end{array}$

$\begin{array}{llll}70.1 & 68.5 & 52.8 & 52.5\end{array}$

.71

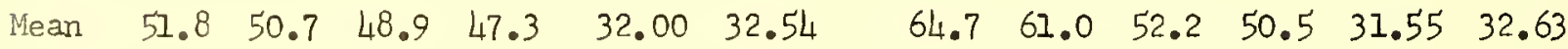


WINTER QUARTER LIGHTSHIP $\quad 37^{\circ} 55^{\prime} 05^{\prime \prime N}$. 74 $54^{\circ} 04^{\prime \prime W}$.

Depth of water - 75 feet

1956

Jury

Temperature ${ }^{\circ} \mathrm{F}$

Salinity $\%$

August

Depth Day $0^{\prime} \quad 30^{\prime} \quad 50^{\prime} \quad 75^{\prime} \quad 0^{\prime} \quad 75^{\prime} \quad 0^{\prime} \quad 30^{\prime} \quad 50^{\prime} 75^{\prime}$ o'

$\begin{array}{llllll}1 & 72.3 & 69.2 & 57.2 & 51.4 & 30.83\end{array}$

$\begin{array}{llllll}2 & 72.1 & 67.3 & 52.1 & 51.7 & 31.72\end{array}$

$\begin{array}{llllll}3 & 73.9 & 70.8 & 67.0 & 52.1 & .54 \\ 4 & 73.9 & 70.3 & 59.1 & 52.5 & .57\end{array}$

$\begin{array}{lllll}4 & 73.9 & 70.3 & 59.1 & 52.5 \\ 5 & 74.8 & 69.0 & 54.8 & 52.7\end{array}$

.51

$-$

73.5

Temperature ${ }^{\circ} \mathrm{F}$

Salinity $\%$

$\begin{array}{llllll}6 & 74.7 & 68.9 & 54.8 & 52.5\end{array}$

$0.55 \quad 32.76$

$\begin{array}{lllll}75.9 & 75.7 & 64.2 & 57.2 & .28\end{array}$

$\begin{array}{ccccc}75.3 & 75.1 & 59.0 & 56.2 & - \\ 73.25\end{array}$

$\begin{array}{llll}73.4 & 70.1 & 56.1 & 53.9\end{array}$

$72.4 \quad 71.4 \quad 59.9 \quad 55.5$

$.00 \quad 32.47$

$\begin{array}{lllll}7 & 73.6 & 65.2 & 53.9 & 53.8\end{array}$

$.34=$

$73.6 \quad 66.4 \quad 62.4$

.19

$\begin{array}{lllll}8 & 73.2 & 60.9 & 54.1 & 53.9\end{array}$

$\begin{array}{lllll}9 & 70.3 & 69.1 & 52.9 & 52.5\end{array}$

.26

$.54 \quad-$

72.

$62.3 \quad 31.09$

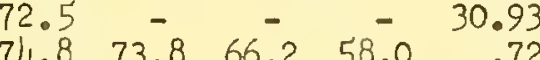

10

$11 \quad 67.3 \quad 60.9 \quad 52.6 \quad 51.5$

- $\quad-$

75.8

58.0

75.60 .68

67.9

58.1

.72

$\begin{array}{lllll}12 & 68.7 & 54.3 & 51.0 & 51.0\end{array}$

.58

$-$

75.4

62.5

56.8

31.11

$13 \quad 68.1 \quad 56.1 \quad 51.1 \quad 51.0$

$\begin{array}{lllll}14 & 67.9 & 58.2 & 51.2 & 59.9\end{array}$

$.75 \quad-$

77.4

71.4

75.5

74.9

60.2

76.4

$68.4-67.6$

56.4

$\begin{array}{lllll}15 & 67.7 & 64.0 & 50.9 & 49.0\end{array}$

$\begin{array}{lllll}16 & 70.9 & 57.5 & 51.7 & 50.9\end{array}$

$\begin{array}{lllll}17 & 70.1 & 59.8 & 5.7 & 51.0\end{array}$

$.38-$

.11

77.2

71.3

60.0

56.4

30.87

31.48

75.

$\begin{array}{lr}56.4 & .92 \\ 57.1 & 30.87\end{array}$

$\begin{array}{lllll}18 & 70.5 & 58.9 & 51.9 & 51.0\end{array}$

$1972.1 \quad 54.2 \quad 51.6 \quad 51.1$

30.65

$.62 \quad 32.67$

80.376.

2070

.95

72.

$\begin{array}{llllll}21 & 71.2 & 67.1 & 52.3 & 51.9 & 30.96\end{array}$

$\begin{array}{llllll}22 & 73.1 & 63.9 & 54.4 & 52.1 & 31.06\end{array}$

$\begin{array}{lllll}23 & 71.2 & 70.0 & 50.7 & 50.2\end{array}$

$\begin{array}{lllll}24 & 70.6 & 69.9 & 52.0 & 50.0\end{array}$

.07

$\begin{array}{lllll}25 & 71.8 & 70.6 & 63.5 & 52.0\end{array}$

$\begin{array}{lllll}26 & 72.2 & 70.2 & 63.0 & 52.2\end{array}$

$\begin{array}{lllll}27 & 72.8 & 56.1 & 52.8 & 52.2\end{array}$

$\begin{array}{rrrrr}28 & 72 . & - & - & - \\ 29 & 72.4 & 56.5 & 52.9 & 51.0\end{array}$

$\begin{array}{lllll}30 & 70.1 & 68.8 & 52.6 & 51.0\end{array}$

$\begin{array}{lllll}31 & 72.7 & 65.2 & 53.1 & 51.7\end{array}$

.14

.27

.18

.36
.40

.16

$.24 \quad-$

73

$\begin{array}{cccccc}- & - & - & - & - & - \\ 73 . & - & - & - & .26 & - \\ 72.5 & - & - & - & .09 & - \\ 77.0 & - & - & - & .18 & - \\ 74 . & - & - & - & .57 & 31.79 \\ 74 . & - & - & - & .20 & - \\ 79.7 & - & - & - & .27 & - \\ 75.5 & - & - & - & .37 & - \\ 77.9 & 76.8 & 69.6 & 58.6 & .49 & - \\ 78.3 & 78.1 & 71.5 & 57.8 & - & - \\ 80.4 & 77.6 & 66.6 & 58.8 & - & -\end{array}$

Mean

$71.4 \quad 64.0 \quad 54.251 .6$

$31.28 \quad 32.66$

$\begin{array}{llllll}75.5 & 73.0 & 64.0 & 57.2 & 31.14 & 32.19\end{array}$ 
WINTER QUARTER LIGHTSHIP

$37^{\circ} 55^{\prime} 05^{\prime \prime N} .74^{\circ} 56^{\prime} 04^{\prime \prime W}$.

Depth of water - September $75 \mathrm{ft}$ 1956

October $95 \mathrm{ft}$.

\section{September}

Temperature ${ }^{\circ} \mathrm{F} \quad$ Salinity $\%$
October

Temperature ${ }^{\circ}$

Salinity $\%$

$0^{\prime} 30^{\prime} 50^{\prime} \quad 95^{\prime} \quad 0^{\prime} \quad 951$

$\begin{array}{lllll}64.2 & 64.2 & 64.1 & 63.8 & 31.24\end{array}$

$64.264 .1 \quad 64.0 \quad 64.1$

$\begin{array}{llll}65.2 & 64.9 & 64.7 & 64.5\end{array}$

$65.1 \quad 65.1 \quad 65.1 \quad 64.2$

$65.165 .1 \quad 65.1 \quad 64.5$

$65.065 .1 \quad 65.0 \quad 65.0$

$\begin{array}{llll}64.6 & 64.7 & 64.7 & 64.5\end{array}$

$64.3 \quad 64.3 \quad 64.2 \quad 64.2$

$60.260 .2 \quad 60.1 \quad 60.1$

$\begin{array}{llll}64.7 & 64.7 & 64.8 & 64.9\end{array}$

$\begin{array}{cc}31.24 & - \\ .22 & - \\ .26 & - \\ .32 & 31.51 \\ .46 & - \\ .39 & - \\ .36 & - \\ .45 & - \\ .54 & - \\ .55 & -\end{array}$

$64.1 \quad 64.1 \quad 64.1 \quad 64.1$

$\begin{array}{llll}63.2 & 63.6 & 63.7 & 63.7\end{array}$

$63.163 .1 \quad 63.1 \quad 63.2$

$\begin{array}{llll}63.1 & 63.1 & 63.2 & 63.2\end{array}$

$\begin{array}{llll}63.6 & 63.1 & 63.0 & 63.0\end{array}$

$\begin{array}{lllll}63.4 & 63.4 & 63.2 & 62.3\end{array}$

$\begin{array}{llll}63.7 & 63.8 & 63.8 & 62.8\end{array}$

$.56 \quad 31.93$

$\begin{array}{lllll}63.4 & 63.5 & 63.5 & 63.6 & 31.94\end{array}$

$\begin{array}{lllll}63.0 & 63.0 & 63.0 & 62.9 & 32.16\end{array}$

$63.163 .1 \quad 63.1 \quad 62.9 \quad .15 \quad-$

$\begin{array}{llllll}63.0 & 63.0 & 63.1 & 63.1 & 32.20 & -\end{array}$

$\begin{array}{lllll}63.3 & 63.1 & 62.9 & 62.9 & 31.97\end{array}$

$\begin{array}{lllll}62.7 & 62.9 & 62.9 & 62.8 & 32.28\end{array}$

$\begin{array}{lllll}61.7 & 61.8 & 61.9 & 61.9 & .33\end{array}$

$\begin{array}{lllll}61.9 & 61.9 & 61.9 & 61.9 & .09\end{array}$

$\begin{array}{lllll}61.2 & 61.3 & 61.3 & 61.3 & .11\end{array}$

$\begin{array}{lllll}60.9 & 61.1 & 61.1 & 61.2 & 32.20\end{array}$

$\begin{array}{lllll}61.1 & 61.2 & 61.5 & 61.5 & 31.93\end{array}$

$61.8 \quad 61.9 \quad 61.9$

62.5

$.93=$
$-\quad 31.65=$

$\begin{array}{lllllll}30 & 64.6 & 64.3 & 64.3 & 64.3 & 31.33 & =\end{array}$

Mean

$71.7 \quad-\quad-$

$31.33 \quad 31.92$

$\begin{array}{llllll}63.2 & 63.2 & 63.2 & 63.1 & 31.77 & 31.92\end{array}$ 
WINTER QUARTER LIGHTSHIP $37^{\circ} 55^{\prime} 05^{\prime \prime N}$. 74056'OL"W. 1956

November
Salinity $\%$

$0^{\prime} \quad 95^{\prime}$

$\begin{array}{lllllll}1 & 61.8 & 61.2 & 61.0 & 60.4 & 31.52 & 32.18\end{array}$

$\begin{array}{lllll}2 & 61.9 & 60.8 & 60.7 & 60.2\end{array}$

$362-1-2$

$\begin{array}{lllll}4 & 61.5 & - & - & - \\ 5 & 60.9 & 60.8 & 60.9 & 60.9\end{array}$

$\begin{array}{lllll}7 & 60.1 & 59.9 & 59.8 & 59.9\end{array}$

$\begin{array}{lllll}8 & 59.6 & 59.9 & 59.5 & 59.6\end{array}$

$\begin{array}{lllll}9 & 59.0 & 59.1 & 59.2 & 59.6\end{array}$

$\begin{array}{lllll}10 & 58.5 & 58.3 & 58.3 & 58.9\end{array}$

$\begin{array}{lllll}11 & 57.9 & 58.1 & 58.5 & 59.0\end{array}$

$\begin{array}{lllll}12 & 58.2 & 58.2 & 58.3 & 58.9\end{array}$

$\begin{array}{lllll}13 & 57.9 & 58.0 & 58.1 & 59.1\end{array}$

$\begin{array}{lllll}14 & 57.9 & 57.9 & 58.1 & 58.4 \\ 15 & 58.1 & 58.1 & 58.9 & 59.1\end{array}$

$\begin{array}{lllll}15 & 58.1 & 58.1 & 58.9 & 59.1 \\ 16 & 58.1 & 58.1 & 58.8 & 60.3\end{array}$

$\begin{array}{lllll}17 & 58.0 & 58.1 & 58.1 & 59.2\end{array}$

$\begin{array}{lllll}18 & 57.8 & 57.9 & 58.1 & 58.2\end{array}$

$\begin{array}{lllll}19 & 57.2 & 57.2 & 57.3 & 57.5\end{array}$

$\begin{array}{lllll}20 & 57.3 & 57.2 & 57.4 & 57.6\end{array}$

$\begin{array}{lllll}21 & 57.3 & 57.4 & 57.9 & 58.0\end{array}$

$\begin{array}{lllll}22 & 57.5 & 57.3 & 57.5 & 57.8\end{array}$

$\begin{array}{lllll}23 & 56.4 & 56.9 & 57.1 & 57.4\end{array}$

$\begin{array}{lllll}24 & 56.3 & 56.6 & 56.8 & 56.9\end{array}$

$\begin{array}{lllll}25 & 55.8 & 55.9 & 55.9 & 56.0\end{array}$

$\begin{array}{lllll}26 & 55.7 & 55.6 & 55.7 & 56.2\end{array}$

$\begin{array}{lllll}27 & 55.8 & 55.7 & 55.9 & 56.0\end{array}$

$28 \quad 54.4 \quad 54.9 \quad 55.1 \quad 56.0$

$29 \quad 54.3 \quad 54.9 \quad 54.9 \quad 55.0$

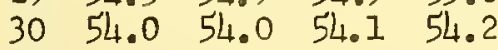

31
$.63-$

$.84 \quad-$

.84

32.01

.06

32.00

$31.93 \quad 32.57$

.93

31.64

32.13

.28

$.24 \quad-$

.5133 .19

.19 -

$.43 \quad-$

.68 -

.84

$.85=$

.6033 .00

.89

.80

.62

.76

.67
.78

.85
$.22 \quad-$
Temperature ${ }^{\circ} \mathrm{F}$

$0^{\prime} \quad 30^{\prime} 50^{\prime} 95^{\prime}$

$53.0 \quad 53.2 \quad 53.6 \quad 53.8$

$\begin{array}{llll}53.0 & 53.2 & 53.4 & 54.0\end{array}$

53.353 .3

53.753 .7

$53.6 \quad 53.7$

$52.5 \quad 53.2$

$52.7 \quad 52.9$

52.853 .0

52.052 .1

$\begin{array}{llll}52.0 & 52.0 & 52.1 & 52.1\end{array}$

$52.0 \quad 52.0 \quad 52.0 \quad 52.1$

$\begin{array}{llll}53.0 & 53.0 & 53.1 & 53.2\end{array}$

$\begin{array}{llll}52.4 & 52.5 & 52.7 & 52.7\end{array}$

$\begin{array}{llll}52.2 & 52.2 & 52.4 & 52.5\end{array}$

$\begin{array}{llll}52.0 & 52.9 & 53.0 \quad 53.2\end{array}$

$53.0 \quad 53.0 \quad 53.1 \quad 53.2$

51.

52.

51.

52.

53.0

53.3

53.0

它. 2 .

52 .

51.5

52.6

2.

52.2

52.0

51.0

50.8

52.1

50.7

50.5

50.7

$\begin{array}{ll}49.8 & 50.1 \\ 50.0 & 50.0\end{array}$

$\begin{array}{llllll}52.0 & 52.3 & 52.7 & 53.2 & 32.52 & 32.94\end{array}$

Salinity $\%$

$$
0^{\prime} \quad 95^{\prime}
$$

$32.60-$

$.68-$

$32.89-$

32.94

$.60 \quad 32.80$

$.47-$

$.079 \quad-$

$.55-$

$\begin{array}{cc}.51 & - \\ .60 & - \\ .90 & 32.82 \\ .60 & - \\ .42 & - \\ .30 & - \\ 32.85 & - \\ 31.81 & - \\ 32.92 & - \\ .27 & 32.98\end{array}$

$52.2 \quad 53.3$

$.15-$

$.14 \quad-$

$.04 \quad-$

$.73-$

.5750 .78

.31

.51 32.37

$\begin{array}{llllll}\text { Mean } \quad 58.0 \quad 57.8 & 57.9 & 58.2 & 32.31 & 32.81\end{array}$ 
Winter surface temperatures tended to be several derrees lower than the 1947-1956 mean. The spring warming lagged behind the mean. Summer surface temperatures tended hi her than the mean except for a depression in mid-July. Autumnal cooling preceeded the mean except for a sudden retardation of cooling; in December.

Minimum temperatures, less than $38^{\circ} \mathrm{F}$, occurred during the last-third of January. During February and March surface temperatures were usually only a fraction of a degree warmer than the bottom temperatures at the surface of reater than $77^{\circ} \mathrm{F}$ were reached in mid-fugust. Maximum temperatures at the bottom of sreater than $72^{\circ} \mathrm{F}$ were reached in mid-Septernber at the commencement of the autumnal overturn.

Surface salinity ransed from $32 \%$ at the beginning of the Jear to less than $26 \%$ in late June followed by an irrezular recovery to $32 \%$ at year end. Bottom salinities tended to be several parts per thousand more saline during most of the year, but during the period of marked turnover in the water column surface and bottom salinities were nearly identical.

The depression in surface temperature in mid-July resulted from an intrusion of cold saline water from offshore which influenced the whole water column. The intrusion appears to follow a period of preponderant strong southerly winds. The winds observed at the lightship were predominantly strons southerly for the first two thirds of July and averared to very light westerlies during the third-third when the deeper water became much warmer. Inasmuch as the mean surface pressure map for the month of July 1956 is almost an exact replica of the long-time normel it may be that the condition which produced the encroachment of cold water is a normal phenomenon. We have no previous data on this subject other than the daily surface water temperature records for previous years. The one-third monthly means for previous years indicate a tendency for a surface water temperature depression at some tine during the summer. This was particularly marked in 1954 when the usual maximum surface temperatures were not reached.

Comparison of the day to day . Muctuations in the temperature at all depths during the summor of 1956 with the wind systems indicated, in eneral, that colder temperatures at the middle and lower depths are assoc'ated with southwesterly winds, while warming occurs with nor th easterly or easterly winds. Presumably the strong southwesterly and westerly winds move the surface water offshore and allow the encroachment of colder more saline water from offshore. Northeasterly and easterly winds on the other hand press the coastal water against the shore and force the deeper water off shore. This is similar to the phenomenon reported by Jongard and Banks (1952) at Sambro Lightship but requires less wind for a shorter duration. Here we have only a two layered system whereas they had a three layered system. 
The influence of other than purely local winds is apparent as a better correlation between water movements and wind is obtained from observing the position of fronts on the sequence of daily weather maps than from the purely local observations on the lightship. This intrusion of offshore water is apparent at Winter Quarter and Five Fathom Bank Lightships to a lesser degree, but at the same times as at Chesapeake Lightship, indicating that this intrusion and regression occurred 211 along this stretch of the coast.
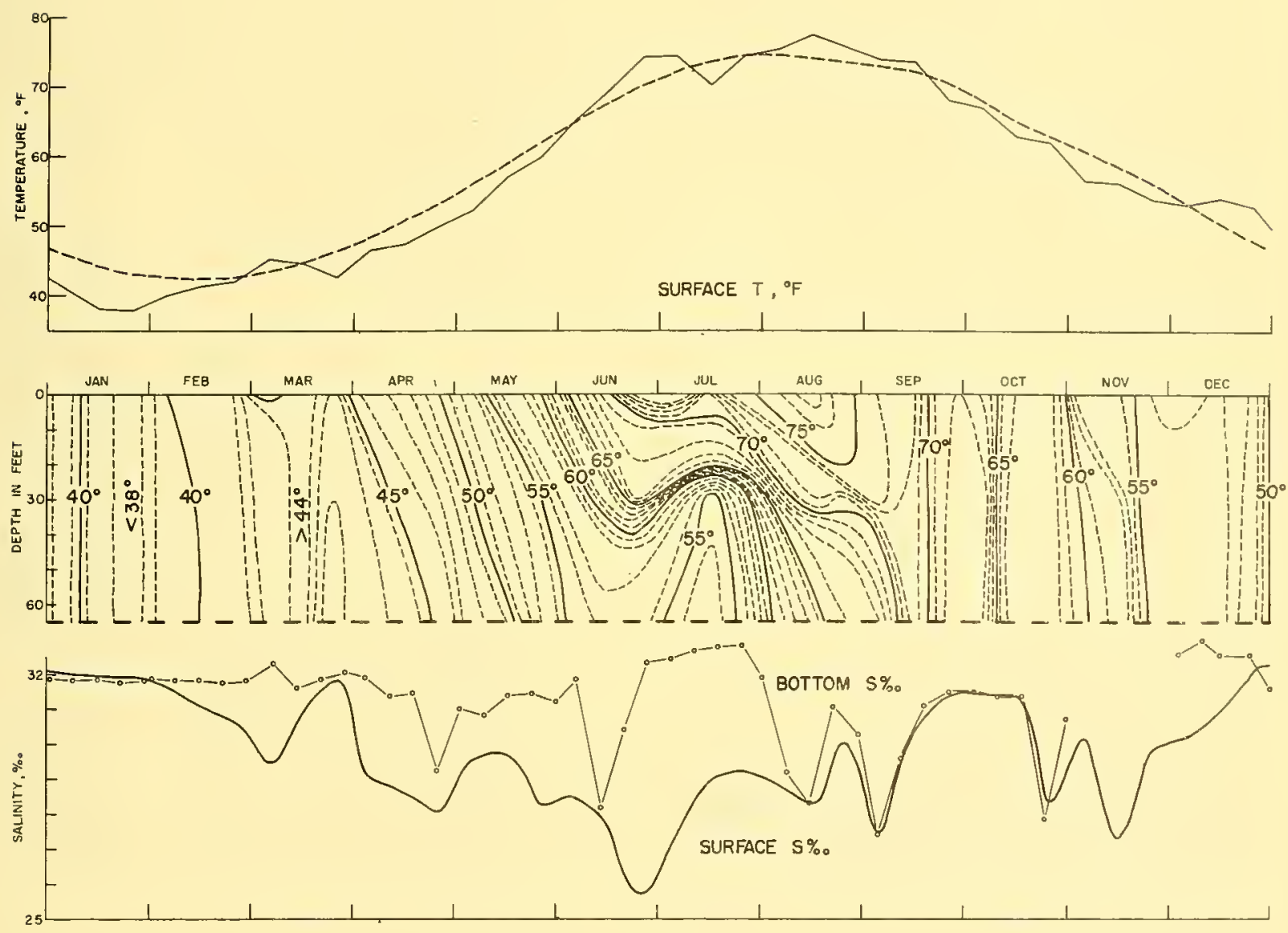

Figure 15. Chesapeake Lightship, 1956. (Dashed line in upper diagram, mean for period 1947-1956) 
Table $\nu_{1}$

CHESAPEAKE LIGHTSHIP

$$
\text { November } 1955
$$

Temperatare ${ }^{\mathrm{O}} \mathrm{F}$

Salinity $\%$

Day

1

2

3

5

6

7
8

9

10

11

12

13

14

15

16

17

18

19

20

21

22

23

24

25

26

27

28

Mean
Temperature ${ }^{\mathrm{O}} \mathrm{F}$

Salinity $\%$

Depth of water - 65 feet

\section{December}

$\begin{array}{llllll}0^{\prime} & 30^{\prime} & 50^{\prime} & 65^{\prime} & 0^{\prime} & 65^{\prime}\end{array}$

$\begin{array}{llllll}50.7 & 52.3 & 52.7 & 52.8 & 30.75 & -\end{array}$

$\begin{array}{llllll}51.1 & 51.9 & 52.7 & 52.8 & 29.70 & -\end{array}$

$\begin{array}{cccccc}51.7 & 52.4 & 52.8 & 52.8 & 28.69 & - \\ 52.2 & 52.7 & 52.9 & 53.0 & 29.65 & 32.19\end{array}$

$\begin{array}{llllll}51.9 & 52.2 & 53.0 & 53.0 & 31.59 & -\end{array}$

$51.8 \quad 51.951 .951 .9 \quad 32.21$ -

$\begin{array}{llllll}50.9 & 51.3 & 51.3 & 51.3 & .32 & -\end{array}$

$50.151 .0 \quad 51.1 \quad 51.1 \quad 0.53 \quad-$

$\begin{array}{lllll}50.1 & 50.1 & 50.1 & 50.2 & .22\end{array}$

$\begin{array}{lllllll}48.8 & 49.5 & 49.7 & 49.7 & .18\end{array}$

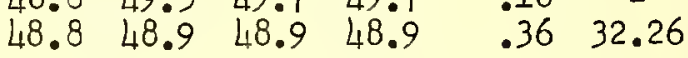

$\begin{array}{llllll}48.3 & 48.2 & 48 . \overline{-} & 48 . \overline{-} & 32 . \overline{-} & 32.16\end{array}$

$\begin{array}{llllll}48.8 & 48.5 & 48.5 & 48.4 & .21 & \text { - }\end{array}$

$\begin{array}{lllll}47.8 & 47.7 & 47.6 & 47.6 & 32.19\end{array}$

$\begin{array}{llllll}43.4 & 47.7 & 48.0 & 48.1 & 29.77 & -\end{array}$

$\begin{array}{lllll}47.0 & 46.8 & 46.8 & 46.8 & -\end{array}$

$\begin{array}{lllll}46.9 & 45.8 & 46.8 & 46.8 & 32.28\end{array}$

$\begin{array}{lllll}46.7 & 46.8 & 46.8 & 46.6 & .28\end{array}$

$\begin{array}{llllll}45.0 & 45.0 & 45.0 & 44.9 & 32.17 & 32.18\end{array}$

$44.9 \quad 44.1 \quad 44.1 \quad 44.1 \quad 33.22 \quad-$

$45.5 \quad 44.9 \quad 44.9 \quad 44.9 \quad 32.22=$

$\begin{array}{llllll}44.6 & 44.2 & 44.1 & 44.1 & .18 & -\end{array}$

$44.944 .9 \quad 45.0 \quad 45.0 \quad .01$ -

$\begin{array}{lllll}44.9 & 44.8 & 44.8 & 44.7 & .28\end{array}$

$\begin{array}{lllll}43.7 & 43.3 & 43.3 & 43.4 & .18\end{array}$

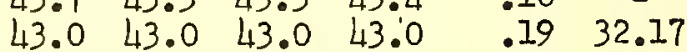

$\begin{array}{llllllll}43.2 & 43.1 & 43.1 & 43.1 & .23 & -\end{array}$

$\begin{array}{lllllll}43.0 & 43.0 & 43.1 & 43.1 & .20 & -\end{array}$

$\begin{array}{lllll}42.6 & 42.5 & 1+2.5 & 1,2.6 & .32\end{array}$

$\begin{array}{llllll}47.4 & 47.7 & 47.8 & 47.8 & 31.76 & 32.25\end{array}$

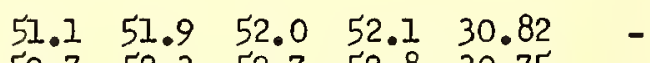


Table 14 ( cont'd)

CHESAPEAKE LIGHTSHIP

January

Temperature ${ }^{\circ}$ $36^{\circ} 58^{\prime} 07^{\prime N} \mathrm{~N} .75^{\circ} 42^{\prime} 02^{\prime \prime w . ~}$

1956
Salinity $\%$

$0^{\prime} 65^{\prime}$

Depth

Day

$\begin{array}{lllll}1 & 41.9 & 42.4 & 42.9 & 42.8\end{array}$

$\begin{array}{lllll}2 & 42.5 & 42.2 & 42.2 & 42.2\end{array}$

$\begin{array}{lllll}3 & 41.9 & 41.9 & 42.0 & 42.1\end{array}$

$\begin{array}{lllll}4 & 42.1 & 42.1 & 42.1 & 42.1\end{array}$

$\begin{array}{lllll}5 & 39.3 & 39.2 & 39.4 & 39.8\end{array}$

$\begin{array}{lllll}6 & 42.5 & 42.9 & 43.0 & 43.0\end{array}$

$\begin{array}{lllll}7 & 42.5 & 42.2 & 42.3 & 42.4 \\ 8 & 40.9 & 40.9 & 40.9 & 40.8\end{array}$

$\begin{array}{lllll}9 & 38.8 & 38.8 & 38.8 & 38.8\end{array}$

$\begin{array}{lllll}10 & 39.4 & 39.1 & 39.1 & 39.1\end{array}$

$\begin{array}{lllll}17 & 39.5 & 39.1 & 39.3 & 40.0\end{array}$

$\begin{array}{lllll}12 & 39.0 & 39.0 & 39.2 & 39.3\end{array}$

$\begin{array}{lllll}13 & 38.5 & 38.1 & 38.1 & 38.2\end{array}$

$\begin{array}{lllll}14 & 37.9 & 37.9 & 37.9 & 37.9\end{array}$

$\begin{array}{lllll}15 & 38.2 & 38.3 & 39.0 & 39.0\end{array}$

$\begin{array}{lllll}16 & 37.6 & 38.0 & 38.5 & 38.6\end{array}$

$\begin{array}{lllll}17 & 37.8 & 37.8 & 37.8 & 37.8\end{array}$

$\begin{array}{lllll}18 & 37.8 & 37.8 & 37.8 & 37.8\end{array}$

$\begin{array}{lllll}19 & 37.7 & 37.7 & 37.7 & 37.8\end{array}$

$\begin{array}{lllll}20 & 37.4 & 37.6 & 37.7 & 37 .\end{array}$

$\begin{array}{lllll}21 & 37.6 & 37.6 & 37.6 & 37.7\end{array}$

$\begin{array}{lllll}22 & 38.0 & 37.2 & 37.5 & 37.4\end{array}$

$\begin{array}{lllll}23 & 38.0 & 37.5 & 37.5 & 37.5\end{array}$

$24 \quad 38.0 \quad 38.0 \quad 38.0 \quad 38.0$

$\begin{array}{lllll}25 & 37.5 & 37.3 & 37.3 & 37.5\end{array}$

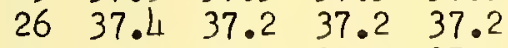

$\begin{array}{lllll}27 & 37.7 & 37.0 & 37.0 & 37.0\end{array}$

$\begin{array}{lllll}28 & 37.5 & 36.8 & 36.8 & 36.9\end{array}$

$\begin{array}{lllll}29 & 37.5 & 37.3 & 37.2 & 37.2\end{array}$

$\begin{array}{lllll}30 & 38.6 & 38.0 & 38.0 & 38.0\end{array}$

$\begin{array}{lllll}31 & 38.2 & 38.2 & 38.2 & 38.2\end{array}$
$32.00-$

$.42=$

.27

32.30

31.95

32.25

32.18

31.84

.37

31.95

32.01

31.80

31.75

32.36

31.87

32.12

31.98

32.00

31.95

$\begin{array}{cc}.93 & - \\ .82 & - \\ 31.95 & - \\ 32.11 & - \\ 31.93 & - \\ 31.98 & - \\ - & 31.93 \\ 31.72 & - \\ .94 & - \\ .70 & - \\ 31.90 & -\end{array}$

February
Temperature ${ }^{\circ} \mathrm{F}$

o' $30^{\prime} 50^{\prime} 65^{\prime}$

$\begin{array}{lllll}39.0 & 38.8 & 38.7 & 38.6\end{array}$

$\begin{array}{llll}39.2 & 39.1 & 39.0 & 38.9\end{array}$

$\begin{array}{llll}39.7 & 39.1 & 39.1 & 39.1\end{array}$

$\begin{array}{llll}39.1 & 38.9 & 38.9 & 38.9\end{array}$

$\begin{array}{llll}39.7 & 39.3 & 39.3 & 39.3\end{array}$

$\begin{array}{llll}39.9 & 39.4 & 39.3 & 39.3\end{array}$

$\begin{array}{llll}39.3 & 39.1 & 39.1 & 39.1\end{array}$

$\begin{array}{llll}41.6 & 39.7 & 39.6 & 39.6\end{array}$

$\begin{array}{llll}41.2 & 39.8 & 39.6 & 39.6\end{array}$

$40.9 \quad 39.6 \quad 39.4 \quad 39.4$

$\begin{array}{llll}40.9 & 39.4 & 39.5 & 39.5\end{array}$

$\begin{array}{llll}39.9 & 39.2 & 39.2 & 39.2\end{array}$

$\begin{array}{llll}41.5 & 39.6 & 39.3 & 39.2\end{array}$

$40.3 \quad 39.7 \quad 39.3$

$44.8 \quad 40.0 \quad 39.9$

39.3

40.8

40.8

40.7
40.2

40.

39.8

40.1

40.8 40.8 $40.7 \quad 40.1$

47.47 .7 4. 40.6

41.

47.1

41.

$\begin{array}{llll}4.8 & 41.3 & 41.3 & 41.2\end{array}$

$\begin{array}{llll}41.4 & 41.2 & 41.3 & 41.2\end{array}$

$41.0 \quad 40.9$

$42.3 \quad 41.3 \quad 41.1$

42.

$42.9 \quad 41.4 \quad 41.1 \quad 41.1 \quad 30.18$

$43.0 \quad 41.8 \quad 41.8 \quad 41.8 \quad 30.51$

42. $\begin{array}{cc}31.90 & 31.89 \\ 31.94 & - \\ 30.27 & - \\ 31.97 & - \\ .89 & - \\ .89 & - \\ .89 & - \\ .83 & 31.85 \\ .62 & - \\ .55 & -\end{array}$

$\begin{array}{cc}.51 & - \\ .55 & - \\ .56 & - \\ 31.56 & - \\ 26.77 & 31.82 \\ 31.47 & - \\ .60 & - \\ .66 & - \\ .73 & - \\ .66 & -\end{array}$

.59

$.72 \quad 31.78$

.78

31.71

$-$

-

$28.75-$
$31.74 \quad 31.81$

Mean

$\begin{array}{llll}39.0 & 38.9 & 39.0 \quad 39.0\end{array}$

$31.97 \quad 32.02$

$\begin{array}{lllll}41.0 & 40.2 \quad 39.1 \quad 40.0 \quad 31.18\end{array}$

31.83 
CHESAPEAKE IIGHTSHIP $36^{\circ} 58^{\prime} 07^{\prime \prime N} .75^{\circ} 42^{\prime} 02^{\prime \prime W}$.

Depth of water - 65 feet

1956

\author{
March \\ Temperature $\mathrm{O}_{\mathrm{F}}$ \\ April \\ Temperature ${ }^{\circ}$ F Salinity $\%$
}

\begin{tabular}{|c|c|c|c|c|c|c|c|c|c|c|c|c|}
\hline$\frac{e p t h}{\text { Day }}$ & $0^{\prime}$ & $30^{\prime}$ & $50^{\prime}$ & $65^{\prime}$ & $0^{\prime}$ & $65^{1}$ & $0^{\prime}$ & $30^{\prime}$ & $50^{\prime}$ & $65^{\prime}$ & $0^{1}$ & $65^{\prime}$ \\
\hline $\begin{array}{r}1 \\
2 \\
3 \\
4 \\
5 \\
6 \\
7 \\
8 \\
9 \\
10\end{array}$ & $\begin{array}{l}42.7 \\
43.4 \\
44.0 \\
43.2 \\
44.4 \\
46.1 \\
47.4 \\
46.0 \\
46.5 \\
47.6\end{array}$ & $\begin{array}{l}42.0 \\
42.0 \\
42.3 \\
43.0 \\
43.1 \\
44.0 \\
46.0 \\
44.3 \\
44.9 \\
44.5\end{array}$ & $\begin{array}{l}41.5 \\
42.0 \\
42.2 \\
42.8 \\
42.9 \\
43.6 \\
45.1 \\
44.0 \\
44.1 \\
44.5\end{array}$ & $\begin{array}{l}41.5 \\
41.9 \\
42.1 \\
42.7 \\
42.8 \\
43.5 \\
43.3 \\
43.5 \\
44.1 \\
44.4\end{array}$ & $\begin{array}{c}31.16 \\
28.57 \\
28.48 \\
30.38 \\
29.78 \\
30.47 \\
.44 \\
30.58 \\
- \\
25.37\end{array}$ & $\begin{array}{c}- \\
- \\
- \\
- \\
- \\
- \\
32.31 \\
- \\
- \\
-\end{array}$ & $\begin{array}{l}43.5 \\
46.1 \\
44.8 \\
47.9 \\
50.5 \\
50.8 \\
48.1 \\
45.0 \\
45.0 \\
45.1\end{array}$ & $\begin{array}{l}41.9 \\
43.1 \\
42.9 \\
42.9 \\
43.0 \\
42.9 \\
42.7 \\
45.0 \\
44.6 \\
43.9\end{array}$ & $\begin{array}{l}41.8 \\
42.7 \\
42.8 \\
42.7 \\
42.8 \\
42.7 \\
42.7 \\
42.9 \\
43.8 \\
43.7\end{array}$ & $\begin{array}{l}47.9 \\
42.7 \\
42.8 \\
42.6 \\
42.8 \\
42.8 \\
42.6 \\
42.9 \\
43.3 \\
43.6\end{array}$ & $\begin{array}{l}32.06 \\
26.114 \\
28.59 \\
29.15 \\
29.04 \\
24.80 \\
27.67 \\
30.27 \\
31.22 \\
31.35\end{array}$ & $\begin{array}{c}- \\
- \\
31.91 \\
- \\
- \\
- \\
- \\
- \\
-\end{array}$ \\
\hline $\begin{array}{l}11 \\
12 \\
13 \\
14 \\
15 \\
16 \\
17 \\
18 \\
19 \\
20\end{array}$ & $\begin{array}{l}45.0 \\
45.9 \\
46.0 \\
46.0 \\
44.8 \\
44.8 \\
44.5 \\
43.9 \\
43.0 \\
43.0\end{array}$ & $\begin{array}{l}44.1 \\
45.0 \\
45.2 \\
45.1 \\
44.8 \\
44.5 \\
43.9 \\
43.8 \\
43.0 \\
43.0\end{array}$ & $\begin{array}{l}44.1 \\
44.9 \\
44.9 \\
44.9 \\
44.8 \\
44.4 \\
43.9 \\
43.7 \\
42.9 \\
43.0\end{array}$ & $\begin{array}{l}44.1 \\
44.9 \\
44.9 \\
44.8 \\
44.8 \\
44.3 \\
43.9 \\
43.7 \\
42.8 \\
42.9\end{array}$ & $\begin{array}{r}27.08 \\
31.06 \\
30.07 \\
30.07 \\
31.79 \\
32.00 \\
.17 \\
.26 \\
.02 \\
32.25\end{array}$ & $\begin{array}{c}- \\
- \\
- \\
31.61 \\
- \\
- \\
- \\
- \\
- \\
-\end{array}$ & $\begin{array}{l}44.4 \\
44.5 \\
44.5 \\
48.6 \\
47.9 \\
49.0 \\
48.0 \\
49.4 \\
48.9 \\
48.1\end{array}$ & $\begin{array}{l}43.7 \\
43.7 \\
43.4 \\
43.7 \\
43.3 \\
46.4 \\
46.5 \\
46.6 \\
47.7 \\
46.8\end{array}$ & $\begin{array}{l}43.3 \\
43.3 \\
43.3 \\
43.6 \\
43.2 \\
43.9 \\
44.0 \\
43.9 \\
44.1 \\
43.9\end{array}$ & $\begin{array}{l}43.2 \\
43.2 \\
43.1 \\
43.5 \\
43.2 \\
43.8 \\
43.9 \\
43.9 \\
44.0 \\
43.9\end{array}$ & $\begin{array}{l}31.25 \\
31.25 \\
31.27 \\
28.10 \\
27.51 \\
27.08 \\
27.08 \\
27.24 \\
27.22 \\
28.15\end{array}$ & $\begin{array}{c}31.34 \\
- \\
- \\
- \\
- \\
- \\
- \\
31.48 \\
- \\
-\end{array}$ \\
\hline $\begin{array}{l}21 \\
22 \\
23 \\
24 \\
25 \\
26 \\
27 \\
28 \\
29 \\
30 \\
31\end{array}$ & $\begin{array}{l}43.0 \\
43.0 \\
43.0 \\
44.9 \\
42.1 \\
42.2 \\
42.0 \\
41.9 \\
41.5 \\
42.0\end{array}$ & $\begin{array}{l}42.8 \\
42.0 \\
42.5 \\
42.1 \\
42.0 \\
41.8 \\
41.8 \\
41.9 \\
41.5 \\
41.7 \\
41.8\end{array}$ & $\begin{array}{l}42.8 \\
42.0 \\
42.0 \\
42.1 \\
41.9 \\
41.6 \\
41.8 \\
41.9 \\
41.5 \\
41.5 \\
41.8\end{array}$ & $\begin{array}{l}42.8 \\
41.9 \\
41.9 \\
42.1 \\
41.8 \\
41.6 \\
41.8 \\
41.9 \\
41.5 \\
47.5 \\
41.8\end{array}$ & $\begin{array}{r}31.91 \\
.86 \\
31.87 \\
29.80 \\
32.24 \\
.22 \\
32.47 \\
- \\
31.93 \\
.92 \\
31.93\end{array}$ & $\begin{array}{c}31.86 \\
- \\
- \\
- \\
- \\
- \\
32.06 \\
- \\
- \\
-\end{array}$ & $\begin{array}{l}48.3 \\
49.0 \\
48.2 \\
47 . \\
48.5 \\
48.0 \\
49.5 \\
54.2 \\
53.7 \\
53.5\end{array}$ & $\begin{array}{r}45.9 \\
45.4 \\
48.0 \\
-5 \\
46.8 \\
45.9 \\
46.1 \\
46.2 \\
46.2 \\
46.5\end{array}$ & $\begin{array}{c}44.2 \\
44.7 \\
45.2 \\
- \\
45.9 \\
45.7 \\
45.7 \\
46.3 \\
45.5 \\
46.5\end{array}$ & $\begin{array}{c}44.2 \\
44.7 \\
45.2 \\
- \\
- \\
45.7 \\
45.7 \\
45.9 \\
45.5 \\
46.5\end{array}$ & $\begin{array}{l}29.28 \\
26.97 \\
28.93 \\
28.89 \\
30.92 \\
29.72 \\
29.13 \\
27.15 \\
25.13 \\
24.39\end{array}$ & $\begin{array}{c}- \\
- \\
- \\
- \\
29.25 \\
- \\
- \\
- \\
- \\
-\end{array}$ \\
\hline
\end{tabular}

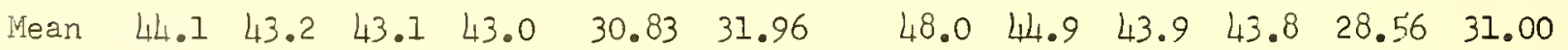




\section{Tale $1 / 4$ (cont'd)}

CHESAPEAKE TIGHTSHIP

$36^{\circ} 58^{\prime} 07^{\prime \prime N}$. 75042'02'W.

Depth of water - 65 feet
May
Salinity $\%$

$0^{\prime}, 65^{\prime}$

Depth

3

5

5

$\begin{array}{lllll}6 & 52.9 & 49.7 & 47.8 & 47.8\end{array}$

$\begin{array}{lllll}7 & 50.7 & 50.6 & 50.3 & 50.2 \\ 8 & 50.5 & 50.5 & 50.3 & 50.2\end{array}$

$9 \quad 52.2 \quad 52.2 \quad 50.4$

1056

$\begin{array}{llllll}17 & 54.0 & 49.6 & 49.1 & 49.1\end{array}$

$\begin{array}{lllll}12 & 59.0 & 50.0 & 49.2 & 49.2\end{array}$

$\begin{array}{lllll}13 & 57.0 & 51.3 & 49.3 & 49.2\end{array}$

$\begin{array}{llll}14 & 61.0 & 51.5 & 49.2 \quad 49.2\end{array}$

$\begin{array}{lllll}15 & 56.0 & 53.6 & 50.7 & 50.5\end{array}$

$\begin{array}{lllll}16 & 56.5 & 51.3 & 50.9 & 50.9\end{array}$

$\begin{array}{lllll}17 & 55.7 & 54.5 & 54.1 & 54.1\end{array}$

$\begin{array}{lllll}18 & 57.0 & 54.2 & 54.1 & 54.1\end{array}$

$\begin{array}{lllll}19 & 58.0 & 54.9 & 54.1 & 54.1\end{array}$

20

\section{2}

$22 \quad 59.8 \quad 53.8$

2364.052 .2

$24 \quad 59.0 \quad 56.3$

$25 \quad 58.358$.

$\begin{array}{lll}26 & 58.8 \quad 57.9\end{array}$

$2760.5 \quad 53$.

$28 \quad 60.3 \quad 53.7$

$\begin{array}{llllll}29 & 58.8 & 53.8 & 53.1 & 53.1\end{array}$

$\begin{array}{lllllll}30 & 58.8 & 55.0 & 53.5 & 53.5 & 29.78 & 31.20\end{array}$

$\begin{array}{lllllll}31 & 60.3 & 53.1 & 52.9 & 52.9 & 28.39 & \end{array}$
1956

June
Temperature ${ }^{\circ} \mathrm{F}$

$0^{\prime} 30^{\prime} \quad 50^{\prime} 65^{\prime}$ o' $65^{\prime}$
Salinity $\%$

$\begin{array}{llllll}66.8 & 55.1 & 54.1 & 54.1 & 24.88 & -\end{array}$

$\begin{array}{lllll}65.1 & 55.1 & 54.1 & 54.1 & 27.23\end{array}$

$63.954 .354 .154 .1 \quad 27.04$ -

$\begin{array}{lllll}65.0 & 54.1 & 54.0 & 54.0 & 28.60\end{array}$

$\begin{array}{lllll}66.7 & 54.9 & 54.9 & 54.8 & 28.85\end{array}$

$\begin{array}{llllll}65.1 & 55.4 & 55.0 & 55.0 & 30.30 & 31.82\end{array}$

$64.9 \quad 62.7 \quad 55.0 \quad 55.029 .43$

$\begin{array}{lllll}65.6 & 56.9 & 55.6 & 55.5 & 28.53\end{array}$

$\begin{array}{lllll}65.0 & 63.1 & 58.2 & 57.5 & 29.84\end{array}$

$\begin{array}{lllll}65.1 & 60.9 & 58.3 & 58.1 & 29.87\end{array}$

$\begin{array}{lllll}66.8 & 59.0 & 56.6 & 56.6 & 30.04\end{array}$

$69 . \quad-\quad-\quad 30.34$

$69.1 \quad 64.4 \quad 55.8 \quad 55.4 \quad 28.45$

$\begin{array}{lllll}71.9 & 58.2 & 55.1 & 55.1 & 26.67\end{array}$

$\begin{array}{lllll}73.0 & 60.3 & 53.9 & 53.5 & 26.49\end{array}$

$68.3 \quad 56.1 \quad 54.5 \quad 54.0 \quad 25.50$

$\begin{array}{lllll}71.4 & 55.1 & 53.3 & 53.3 & 25.79\end{array}$

$\begin{array}{lllll}68.9 & 68.9 & 59.9 & 59.0 & 23.04\end{array}$

$\begin{array}{lllll}68.8 & 58.9 & 59.8 & 59.0 & 30.23\end{array}$

$\begin{array}{lllll}68.1 & 67.7 & 66.8 & 66.5 & 30.25\end{array}$

$\begin{array}{lllll}70.5 & 66.2 & 64.1 & 61.8 & 26.36\end{array}$

$\begin{array}{lllll}71.8 & 65.3 & 59.2 & 58.5 & 23.92\end{array}$

$\begin{array}{lllll}74.9 & 66.5 & 54.7 & 56.6 & 23.42\end{array}$

$\begin{array}{lllll}73.6 & 67.3 & 56.1 & 56.1 & 25.48\end{array}$

$\begin{array}{lllll}77.4 & 67.5 & 56.3 & 56.2 & 24.52\end{array}$

$\begin{array}{lllll}75.8 & 65.6 & 55.8 & 54.8 & 27.71\end{array}$

$\begin{array}{lllll}76.6 & 66.8 & 55.7 & 55.5 & 24.45\end{array}$

75. $\quad-55.4 \quad 55.029 .09$

$\begin{array}{lllll}72.9 & 69.7 & 55.1 & 54.9 & 22.12\end{array}$

$\begin{array}{lllll}73.8 & 70.5 & 56.1 & 55.9 & 28.92\end{array}$
28.16

-

$-$

$-$

$-$

$-$

$-$

30.39

-

$-$

-

$-$

32.30

-

$-$

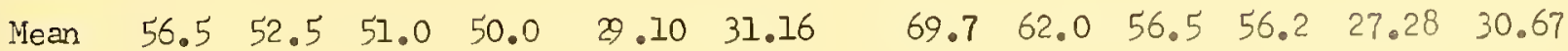


Table I4 (cont'd)

CHSAPEAKE LIGHTSHIP $36^{\circ} 58^{\prime} 07^{\prime \prime N} .75^{\circ} 42^{1} 02^{\prime w}$.

1956

\begin{tabular}{|c|c|c|}
\hline & & Aus \\
\hline Temperature ${ }^{\circ} F$ & Salinity $\% / 00$ & Temperature ${ }^{\circ} F$ \\
\hline
\end{tabular}

Depth

Day $0^{\prime} \quad 30^{\prime} \quad 50^{\prime} 65^{\prime} \quad 0^{\prime} \quad 65^{\prime}$

$\begin{array}{lllllll}1 & 70.3 & 66.2 & 53.1 & 52.6 & 29.43 & \text { - }\end{array}$

$\begin{array}{llllll}2 & 79.3 & 57.8 & 56.1 & 55.8 & 24.15\end{array}$

$\begin{array}{llllll}3 & 79.1 & 60.0 & 56.6 & 55.9 & 23.95\end{array}$

$\begin{array}{lllll}4 & 78.8 & 64.254 .8 & -23.76\end{array}$

$\begin{array}{llllll}5 & 78.1 & 66.1 & 55.3 & 54.9 & 27.49\end{array}$

$\begin{array}{llllll}6 & 75.5 & 57.7 & 55.1 & 55.0 & 28.93\end{array}$

$\begin{array}{llllll}7 & 75.0 & 56.0 & 55.7 & 55.7 & 27.88\end{array}$

$\begin{array}{llllll}8 & 75.7 & 56.3 & 56.0 & 56.0 & 28.39\end{array}$

$\begin{array}{llllll}9 & 66.2 & 54.7 & 54.5 & 54.4 & 29.16\end{array}$

$\begin{array}{llllll}10 & 66.0 & 54.4 & 54.1 & 54.1 & 29.71\end{array}$

$\begin{array}{llllll}11 & 69.8 & 54.6 & 53.2 & 53.2 & 30.08\end{array}$

$\begin{array}{llllll}12 & 70.0 & 54.3 & 53.3 & 53.3 & 28.52\end{array}$

$\begin{array}{lllll}13 & 72.2 & 54.8 & 53.6 & 53.4\end{array}$

$14 \quad 65.3 \quad 54.8 \quad 54.1 \quad 54.1$

$\begin{array}{lllll}15 & 69.6 & 55.1 & 53.9 & 53.1\end{array}$

$\begin{array}{llllll}16 & 71.4 & 53.6 & 53.1 & 53.0\end{array}$

$\begin{array}{llllll}17 & 70.7 & 53.8 & 53.8 & 53.8\end{array}$

$\begin{array}{lllll}18 & 70.0 & 53.6 & 53.3 & 53.3\end{array}$

$\begin{array}{llllll}19 & 72.0 & 55.0 & 54.6 & 54.5\end{array}$

$\begin{array}{lllll}20 & 73.2 & 55.7 & 55.1 & 55.0\end{array}$

$27 \quad 71$

2270.3

$23 \quad 71.5$

2473.7

2572.8

$\begin{array}{lllll}26 & 74.4 & 54.3 & 54.0 & 54.1\end{array}$

$\begin{array}{llllll}27 & 75.3 & 55.0 & 55.1 & 55.0 & 26.38\end{array}$

$\begin{array}{lllllll}28 & 76.9 & 59.6 & 58.2 & 58.0 & 30.09\end{array}$

$\begin{array}{llllll}29 & 76.8 & 62.0 & 58.5 & 58.4 & .06\end{array}$

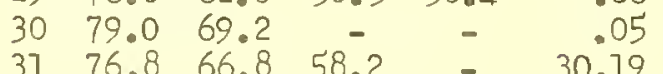

$65^{\prime}$

$-$

2.43

-

$-$

- $\quad 75.7$

$-$

$-$

$-$

32.66

27.62

30.16

27.37

27.32

30.77
28.85

28.96

29.00

29.18

29.09

28.86

28.34

30.19
-
-
-
-
-
32.75
-
-

$-$

$-$

$-$

$-$

$-$

$-$

$-$

$-$

$-$
32.81 $0^{\prime} 30^{\prime} 50^{\prime} 65^{\prime} \quad 0^{\prime} 65^{\prime}$

$75.166 .057 .6-29.1031 .87$

$75.361 .259 .0-30.56-$

$\begin{array}{llllll}75.4 & 60.6 & 59.2 & - & .54 & - \\ 74.9 & 64.2 & - & - & .44 & -\end{array}$

$75.560 .759 .5=30.52=$

$\begin{array}{lccccc}75.4 & - & - & - & 29.20 & - \\ 75.7 & 72.4 & 69.7 & 64.1 & 26.88 & -\end{array}$

$75.973 .9 \quad-\quad-27.54 \quad 29.16$

$\begin{array}{llllll}75.8 & 72.0 & 69.6 & 69.1 & 27.21 & =\end{array}$

$75 . \quad-\quad-26.89$ -

76. - - - .85 -

76. - - $-27.85-$

$77.074 .167 .6-31.95 \quad-$

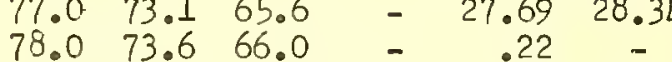

$\begin{array}{llllll}79.1 & 70.7 & 66.9 & 65.5 & .58 & .58\end{array}$

$\begin{array}{lllll}79.4 & 70.365 .3 & - & .58 & -\end{array}$

$79.074 .4-\quad-27.79-$

$77.168 .062 .4-29.27=$

$\begin{array}{cccccc}77.1 & 66.9 & 63.0 & 62.6 & .24 & - \\ 75.8 & 72.0 & 70.2 & - & 29.75 & 31.09\end{array}$

$75.172 .1-2-30.08-$

$\begin{array}{llllll}75.9 & 72.1 & 71.0 & 70.9 & 29.56 & -\end{array}$

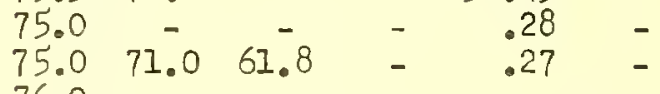

$\begin{array}{llllll}76.0 & - & - & - & - & - \\ 76.0 & 67.0 & 64.8 & - & .30 & 30.28\end{array}$

$76.0-\quad-\quad-\quad .27$

$75.5-\quad-\quad-30.30$
76. - - - $-27.82-$

$75.371 .0-\quad-30.45-$

Mean $73.1 \quad 57.4 \quad 54.8 \quad 54.5 \quad 28.48 \quad 32.66 \quad 76.2 \quad 69.4 \quad 64.7 \quad-\quad 29.07 \quad 30.15$ 
Table $14(\operatorname{con} t a)$

CHESAPEAKE LIGHTSHIP

$36^{\circ} 58^{\prime} 07^{\prime \prime N} \mathrm{~N} .75^{\circ} 42^{\prime} 02^{\prime \prime}$ 沙。

Depth of water - 65 feet

1956

September

Temperature ${ }^{\circ} \mathrm{F}$
Salinity $\%$

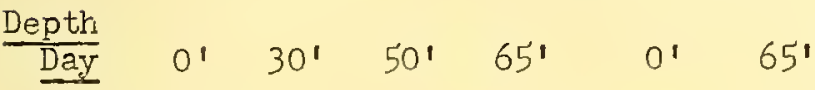

$\begin{array}{rrrcccc}1 & 74.0 & 74.0 & - & - & 27.42 & - \\ 2 & 73.9 & 74.0 & - & - & 27.45 & - \\ 3 & 74.0 & 74.0 & 74.0 & - & 27.32 & - \\ 4 & 74.0 & 74.0 & - & - & 27.38 & - \\ 5 & 74.0 & - & - & - & 27.48 & 27.46 \\ 6 & 74.0 & - & - & - & 27.56 & - \\ 7 & 74.0 & 74.0 & - & - & 27.39 & - \\ 8 & 73.5 & - & - & - & 27.48 & - \\ 9 & 74.5 & 74.3 & - & - & 27.47 & - \\ 10 & 74.2 & 74.1 & 73.8 & - & 27.38 & -\end{array}$

$1774.0 \quad-\quad-\quad-\quad-27.39$

$\begin{array}{llllll}12 & 73.3 & 73.5 & 73.1 & 73.1 & 29.69\end{array}$

$1374.0 \quad-\quad-\quad-\quad 27.44$

$\begin{array}{llllll}14 & 75.3 & 73.1 & 73.0 & 72.8 & 30.72\end{array}$

$15 \quad 71.5 \quad 71.171 . ? 71.1 \quad 31.26$

16 - $\quad$ - $\quad$ - 31.25

17 - $\quad$ - $\quad$ - 31.24

18 - $-\quad-\quad-31.24$

1

20

2

22

23

.

$24 \quad 68.168 .068 .068 .0 \quad 31.08$

$\begin{array}{llllll}25 & 68.1 & 68.0 & 68.0 & 68.0 & 31.49\end{array}$

$26 \quad 68.1 \quad 68.1 \quad 68.1 \quad 68.1 \quad 31.49$

$\begin{array}{llllll}27 & 68.5 & 68.1 & 68.1 & 68.0 & 31.5 ?\end{array}$

$\begin{array}{llllll}28 & 68.0 & 67.8 & 67.9 & 67.9 & 31.50\end{array}$

$29-31.51$

$\begin{array}{llllll}30 & 68.1 & 68.1 & 68.1 & 68.1 & 31.51\end{array}$

31
29.60

$-$

$-$

$-$

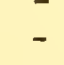

$-$

31.08

$-$

$-$

$-$

$-$

$-$

$3 \overline{1.48}$

$-$

$-$

$-$

$-$

Mean

$\begin{array}{lllll}72.0 & 71.3 & 70.7-69.3-29.69 & 29.90\end{array}$

October

Temperature ${ }^{\circ}$

Salinity $\%$ $\begin{array}{cccccc}68.9 & 68.8 & 68.1 & 68.0 & 31.40 & - \\ 68.9 & 68.9 & 68.1 & 68.0 & .39 & - \\ 68.9 & 68.9 & 68.1 & 68.0 & .38 & 31.48 \\ 67 & - & - & - & .41 & - \\ 67 & - & - & - & .39 & - \\ 67 & - & - & - & .46 & - \\ 66 & - & - & - & .54 & - \\ 66 & - & - & - & .45 & - \\ 67 & - & - & - & .41 & - \\ 63 & - & - & - & .39 & 31.39\end{array}$

$\begin{array}{llllll}62.8 & 62.7 & 62.7 & 62.5 & .36 & -\end{array}$

$\begin{array}{llllll}62.7 & - & - & - & .100 & = \\ 61 . & - & - & - & .36 & - \\ 63 . & - & - & - & .37 & - \\ 62.6 & 62.6 & - & - & .37 & - \\ 62.7 & 62.7 & - & - & .37 & = \\ 64 & - & - & - & .39 & 31.37 \\ 62.8 & 62.8 & - & - & .39 & - \\ 63 & - & - & - & .45 & - \\ 62.9 & - & - & - & .64 & -\end{array}$

64 - $\quad-\quad 31.73$ -

64 - $-\quad-27.92$ -

$\begin{array}{cccccc}62.8 & 62.5 & - & - & .92 & - \\ 61 & - & - & - & .86 & 27.85\end{array}$

$62.8 \quad-\quad-\quad-\quad-\quad-8827.85$

61 - -27.92 -

61 - $-\quad-28.19$ -

$61-\quad-28.06$ -

$62-\quad-27.99-$

62.8 - $-\quad-28.01$ -

62 - $\quad-\quad 28.0330 .73$ 
Table 14 (cont'd)

CHESAPEAKE LIGHTSHIP

$36^{\circ} 58^{\prime} 07^{\prime \prime N} .75^{\circ} 4^{\prime} 02^{\prime \prime W}$.

Depth of water -65 feet 1956

November

December

Temperature ${ }^{\circ} \quad$ Salinity $\%$

Temperature ${ }^{\circ} \mathrm{F}$

Salinity $\%$

$\frac{\text { Depth }}{\text { Day }} \quad 0^{\prime} \quad 30^{\prime} \quad 50^{\prime} \quad 65^{\prime} \quad 0^{\prime} \quad 65^{\circ} \quad 0^{\prime} \quad 30^{\prime} \quad 50^{\prime} \quad 65^{\prime} \quad 0^{\prime} \quad 65^{\prime}$

$\begin{array}{rrrrrrr}1 & 57 . & - & - & - & 30.37 & - \\ 2 & 56 . & - & - & - & .26 & - \\ 3 & 55 . & - & - & - & .10 & - \\ 4 & 59.4 & 61.7 & 60.2 & - & .09 & - \\ 5 & 58.0 & - & - & - & .09 & - \\ 6 & 56 . & - & - & - & .01 & - \\ 7 & 55 . & - & - & - & .08 & - \\ 8 & 58.2 & 58.1 & - & - & .00 & - \\ 9 & 55.0 & - & - & - & .07 & - \\ 10 & 57.3 & - & - & - & .07 & -\end{array}$

$\begin{array}{lllllll}11 & 58.0 & 58.2 & - & - & .06 & - \\ 12 & 58.0 & - & - & - & .07\end{array}$

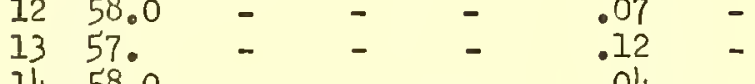

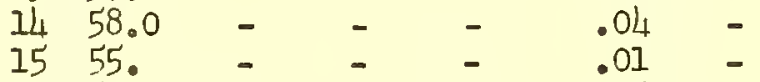

$1656 . \quad-\quad-16=$

$1755 . \quad-\quad-30.14$

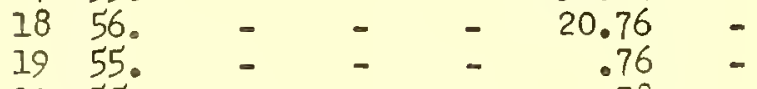

20 55. - - - $\quad$ -

21 55. - - - 20.72 -

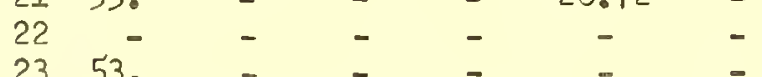

24 53. - - -

$\begin{array}{lllllll}25 & 55.2 & 55.1 & 55.1 & 55.1 & - & -\end{array}$

$\begin{array}{lllllll}26 & 55.7 & 55.9 & 55.9 & 55.9 & - & - \\ 27 & 54.9 & 55.1 & 55.2 & 55.2 & -\end{array}$

$\begin{array}{lllllll}28 & 53.8 & 53.6 & 53.8 & 53.8 & -\end{array}$

$\begin{array}{lllllll}29 & 52.0 & 54.8 & 55.3 & 55.4 & - & - \\ 30 & 53.8 & 53.8 & 53.8 & 53.8 & - & -\end{array}$

31

Mean $55.7 \quad 56.3$ -

$53.5 \quad 54.0 \quad 54.7 \quad 54.8$

52.953 .054 .054 .0 -

52.054 .154 .254 .2

$53.0 \quad 54.4 \quad 54.4 \quad 54.4$

53.554 .254 .154 .2

$53.0 \quad 54.4 \quad 54.4 \quad 54.4$

$53.0 \quad 54.9 \quad 54.9 \quad 54.8$

$\begin{array}{llll}54.0 & 54.1 & 55.1 & 55.2\end{array}$

$53.8 \quad 55.0 \quad 55.5 \quad 55.5$

54.254 .454 .354 .3 -

$\begin{array}{llll}54.0 & 54.7 & 54.7 & 54.7\end{array}$

$54.0 \quad 54.8 \quad 54.954 .9$

$53.0 \quad 54.5 \quad 54.5 \quad 54.5$

$53.7 \quad 55.0 \quad 54.9 \quad 54.9$

54.554 .354 .354 .3

$\begin{array}{llll}54.9 & 54.5 & 54.5 & 54.5\end{array}$

$54.5 \quad 54.6 \quad 54.6 \quad 54.6$

$54.0 \quad 53.9 \quad 54.0 \quad 53.9$

$54.0 \quad 54.7 \quad 54.154 .1$

$\begin{array}{llll}54.8 & 54.8 & 54.8 \quad 54.8\end{array}$

$54.754 .8 \quad 54.5 \quad 54.5$

$54.5 \quad 54.5 \quad 54.4 \quad 54.4$

$\begin{array}{llll}58.4 & - & - & -\end{array}$

$\begin{array}{llll}52.2 & 52.3 & 52.3 & 52.2\end{array}$

51.251 .251 .251 .2

51.251 .951 .951 .9

$\begin{array}{llll}50.1 & 50.2 & 50.3 & 50.3\end{array}$

$\begin{array}{lllll}50.0 & 50.0 & 50.0 & 50.1 & -\end{array}$

$53.4 \quad 53.8 \quad 53.9 \quad 53.9 \quad-$ 
Temperature and salinity fluctuations from day to day are extreme at this location, owing to the fact that the western edge of the Florida Current tends to creep in over the shelf at this point. Meanders in the current sweep to and fro across the position of the lightship.

One-third monthly means of surface temerature indicate that temperatures were lower during the first four months of the year than for the period 1923-56 except for the mid-and last-thirds if February. Autum temperatures were also below the record mean. In other words coastal water tended to extend out across Diamond Shoals during the winter, early spring and autumn to a broader extent than indicated by the average for the period of record for these periods durin the year.

Due to the large range in temperature changes, the depth. time profile of temperature is drawn with five derree isotherms, rather than one degree isotherms as in the other figures. This ficure may be very misleading, however, because the sequence of encroachments of warm saline water sweep in and out again wi th great rapidity. Further, the penetrations of warm water may occur at the surface, midedepth or bottom. Averaging the data for ten days at the other stations simplifies the presentation wi thout severely hiding the significant facts, but may do so at this location. The reader, if interested in the phenomena at Diamond Shoals would do well to draw his own plot from the tabulated daily data.

Surface temperatures ranged from $40-85^{\circ} \mathrm{F}$, bottom temperatures from 42 to $79^{\circ} \mathrm{F}$.

Surface salinity ranged from $29.6 \%$ to $36.3 \%$ and bottom salinity from $32.6 \%$ to $36.4 \%$. 

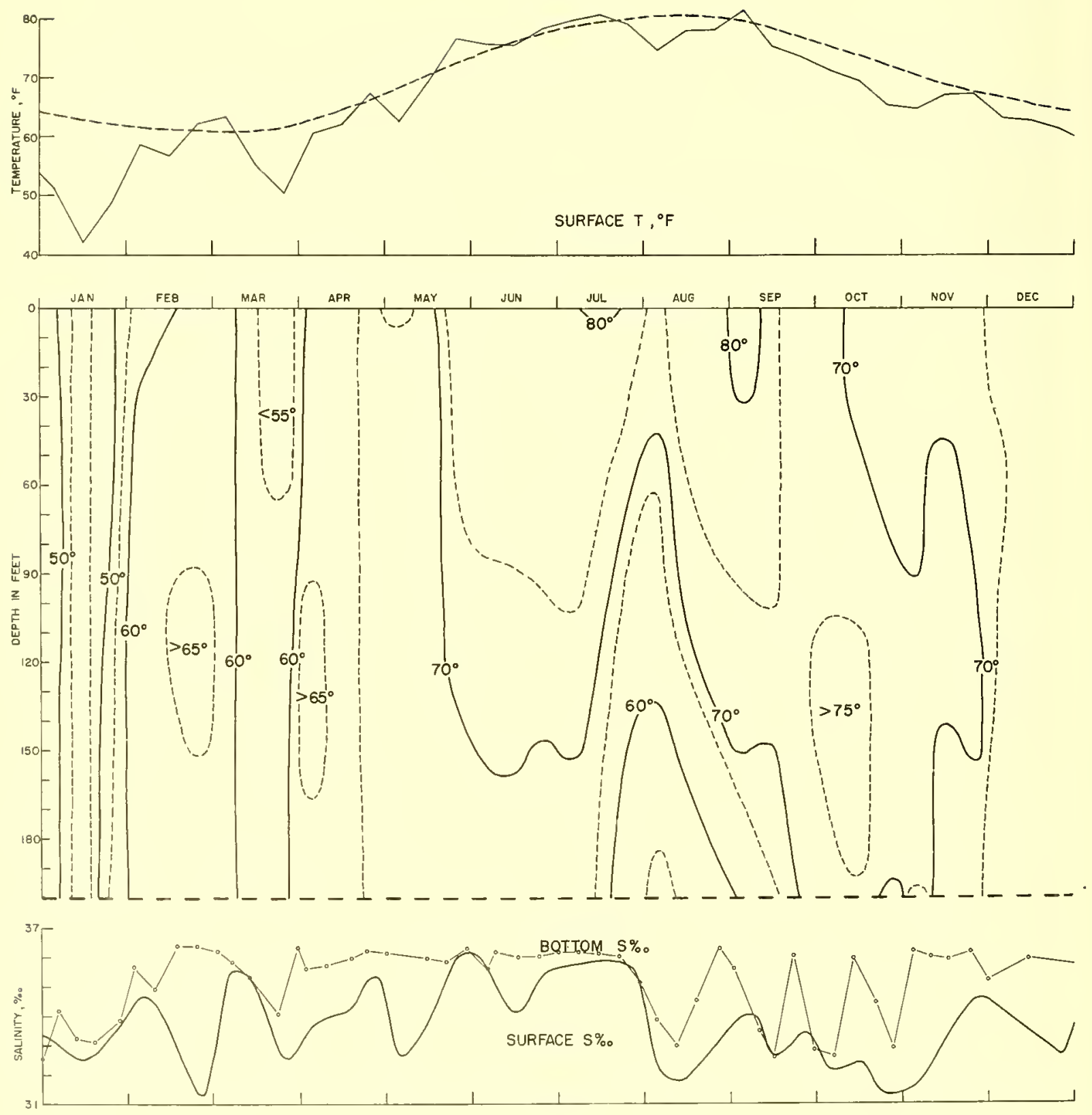

Figure 16. Diamond Shoals Iightship, 1956. (Dashed line in upper diagram, mean for period 1923-1956) 
DIAMOND SHOALS LIGHTSHIP $35^{\circ} 05^{1} 30^{\prime \prime N}$. $75^{\circ} 19^{1} 30^{\prime \prime}$. Depth of water December, 1955

200 feet

Temperature OF

Depth

Day $0^{\prime} 30^{\prime} 50^{\prime} 100^{\prime} 150^{\prime} 200^{\prime}$

$\begin{array}{lllllll}1 & 72.1 & 72.1 & 72.1 & 72.0 & 70.3 & 68.8\end{array}$

$\begin{array}{lllllll}2 & 70.2 & 70.2 & 70.0 & 68.7 & 68.3 & 68.2\end{array}$

$\begin{array}{lllllll}3 & 75.1 & 74.3 & 72.4 & 70.7 & 68.0 & 67.2\end{array}$

$\begin{array}{lllllll}4 & 74.4 & 74.3 & 72.0 & 70.7 & 69.4 & 68.9\end{array}$

$\begin{array}{lllllll}5 & 69.6 & 69.7 & 69.7 & 69.3 & 66.8 & 66.5\end{array}$

$\begin{array}{lllllll}6 & 67.2 & 67.3 & 67.7 & 68.4 & 69.6 & 69.6\end{array}$

$\begin{array}{lllllll}7 & 60.2 & 60.2 & 60.2 & 66.4 & 67.4 & 67.7\end{array}$

$\begin{array}{lllllll}8 & 59.2 & 59.2 & 59.3 & 59.9 & 64.3 & 65.3\end{array}$

$\begin{array}{lllllll}9 & 77.2 & 77.2 & 77.2 & 77.2 & 76.4 & 66.2\end{array}$

$\begin{array}{lllllll}10 & 76.8 & 76.8 & 76.8 & 76.7 & 75.9 & 75.9\end{array}$

$\begin{array}{lllllll}11 & 75.1 & 75.1 & 73.7 & 72.3 & 71.3 & 71.1\end{array}$

$\begin{array}{lllllll}12 & 73.1 & 73.1 & 73.0 & 72.8 & 71.9 & 70.0\end{array}$

$\begin{array}{lllllll}13 & 70.9 & 63.5 & 64.3 & 70.2 & 70.9 & 67.5\end{array}$

$\begin{array}{lllllll}14 & 66.1 & 66.1 & 66.1 & 66.4 & 67.4 & 67.5\end{array}$

$\begin{array}{lllllll}15 & 60.2 & 64.3 & 69.8 & 70.2 & 69.9 & 69.9\end{array}$

$\begin{array}{lllllll}16 & 55.2 & 55.3 & 55.7 & 62.2 & 67.8 & 68.0\end{array}$

$\begin{array}{lllllll}17 & 49.8 & 57.4 & 61.9 & 62.3 & 68.1 & 68.1\end{array}$

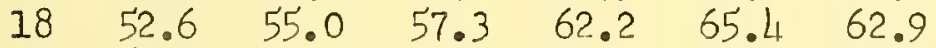

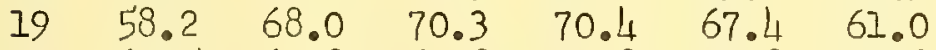

$\begin{array}{lllllll}20 & 60.5 & 60.8 & 60.8 & 60.8 & 60.8 & 60.8\end{array}$

$\begin{array}{lllllll}21 & 52.0 & 52.0 & 52.3 & 56.0 & 59.1 & 60.1\end{array}$

$\begin{array}{lllllll}22 & 50.6 & 50.8 & 51.2 & 52.0 & 57.3 & 60.5\end{array}$

$\begin{array}{lllllll}23 & 52.2 & 54.1 & 54.1 & 63.7 & 64.7 & 64.9\end{array}$

$\begin{array}{lllllll}24 & 51.8 & 56.7 & 59.0 & 59.9 & 66.4 & 66.0\end{array}$

$\begin{array}{lllll}25 & 73.8 & 73.2 & 73.1 & 70.8\end{array}$

$\begin{array}{lllll}26 & 73.5 & 73.7 & 73.8 & 73.9\end{array}$

69.5

73.4

68.8

68.8

$72.1 \quad 72.1 \quad 72.1$

72.1

28

29 30 31
71.5

$47.377^{-} 3 \quad$ -

48.4

55.6

167

57.4

46.4

68.6

46.4

58.3

$\begin{array}{lll}47.5 & 47.5 \quad 47.4\end{array}$
$47.3 \quad 47.3$
Salinity $\%$

$0^{\prime} \quad 200^{\prime}$

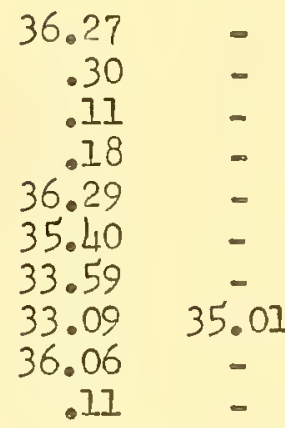

$\begin{array}{lc}.14 & = \\ 36.19 & = \\ 34.10 & = \\ 35.03 & 35.53 \\ 33.66 & = \\ 32.65 & = \\ 31.36 & = \\ 31.81 & = \\ 33.41 & = \\ 34.14 & =\end{array}$

$32.50 \quad 34.23$

32.30 -

$32.37=$

$32.08-$

36.13 -

$.15=$

$36.13=$

$32.40 \quad 32.56$

31.82 -

33.37 -

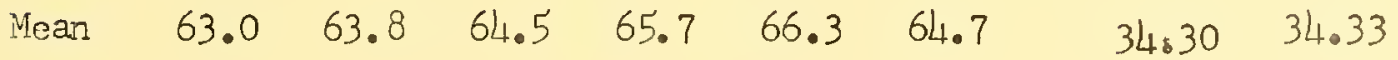


Table 15 (cont'd)

DIAMOND SHOALS LICHTSHIP $35^{\circ} 05^{\prime} 30^{\prime \prime N} \cdot 7^{\circ} 19^{1} 30^{\prime \prime} \mathrm{W}$. Depth of water -

January, 1956

Temperature ${ }^{\circ} \mathrm{F}$

Salinity $\%$

Depth

Day $\quad 0^{\prime} \quad 30^{\prime} \quad 50^{\prime} 100^{\prime} 150^{\prime} 200^{\prime} \quad 0^{\prime} 200^{\prime}$

$\begin{array}{lllllll}1 & 43.7 & 48.1 & 47.8 & 50.7 & 53.2 & 53.3\end{array}$

$\begin{array}{llllll}51.9 & 64.1 & 63.7 & 63.0 & 54.8 & 54.0 \\ 48.1 & 48.1 & 50.1 & 51.3 & 50.9 & 50.0\end{array}$

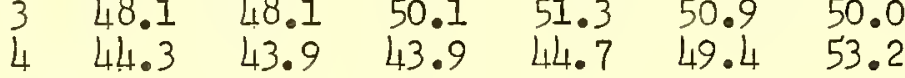

$\begin{array}{ccccccc}5 & - & - & - & - & - & -\end{array}$

$\begin{array}{lllllll}7 & 71.1 & 71.1 & 66.4 & 59.2 & 55.8 & 51.9\end{array}$

8

$\begin{array}{lllllll}9 & 44.8 & 44.7 & 44.7 & 44.8 & 44.9 & 44.9\end{array}$

$\begin{array}{lllllll}10 & 43.6 & 43.3 & 43.4 & 43.9 & 45.4 & 45.7\end{array}$

$\begin{array}{lllllll}11 & 43.4 & 43.4 & 43.4 & 43.7 & 44.0 & 44.1\end{array}$

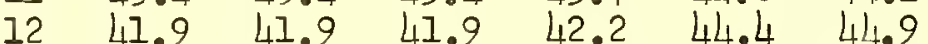

$\begin{array}{lllllll}13 & 41.1 & 41.0 & 41.1 & 41.7 & 43.1 & 43.9\end{array}$

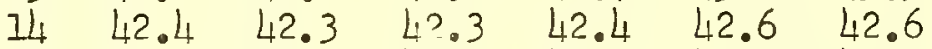

$\begin{array}{lllllll}15 & 43.2 & 42.9 & 43.0 & 42.9 & 43.0 & 43.0\end{array}$

$\begin{array}{lllllll}16 & 43.9 & 43.9 & 43.8 & 43.8 & 43.7 & 43.3\end{array}$

$\begin{array}{lllllll}17 & 40.8 & 40.7 & 41.9 & 43.1 & 43.4 & 43.3\end{array}$

$\begin{array}{lllllll}18 & 42.1 & 41.9 & 41.9 & 41.9 & 42.3 & 44.2\end{array}$

$\begin{array}{lllllll}19 & 43.5 & 43.2 & 43.2 & 43.2 & 43.1 & 43.1\end{array}$

$\begin{array}{lllllll}20 & 40.8 & 40.7 & 41.3 & 43.4 & 43.7 & 43.5\end{array}$

$\begin{array}{lllllll}21 & 41.8 & 41.8 & 41.9 & 42.6 & 42.9 & 43.1\end{array}$

$\begin{array}{lllllll}22 & 42.1 & 42.1 & 42.2 & 42.3 & 42.3 & 42.3\end{array}$

23 - $\quad$ - $\quad$ - $\quad$ - $\quad$ -

$24 \quad \begin{array}{llllll}24 & - & - & - & - & -\end{array}$

$\begin{array}{lllllll}26 & 69.0 & 69.0 & 69.0 & 66.2 & 63.1 & 61.0\end{array}$

$\begin{array}{lllllll}27 & 41.4 & 41.3 & 43.9 & 52.8 & 55.1 & 53.9\end{array}$

$\begin{array}{lllllll}28 & 40.7 & 40.8 & 47.2 & 43.8 & 47.8 & 55.0\end{array}$

$\begin{array}{lllllll}29 & 39.1 & 40.2 & 42.9 & 57.1 & 57.8 & 57.8\end{array}$

$\begin{array}{lllllll}30 & 64.9 & 64.8 & 64.7 & 64.3 & 63.6 & 63.3\end{array}$

$\begin{array}{lllllll}31 & 61.2 & 61.2 & 61.2 & 61.3 & 61.1 & 64.6\end{array}$

$\begin{array}{cc}32.97 & - \\ 32.29 & 33.25 \\ 31.98 & - \\ 32.84 & - \\ 33.16 & - \\ 33.13 & - \\ 31.16 & - \\ 33.72 & 33.14 \\ 33.06 & - \\ 31.92 & -\end{array}$

$32.52-$

$32.88-$

$\begin{array}{cc}- & - \\ 31.55 & - \\ 36.28 & - \\ 32.02 & 33.91 \\ 32.27 & - \\ 32.12 & - \\ 35.73 & - \\ 35.33 & -\end{array}$

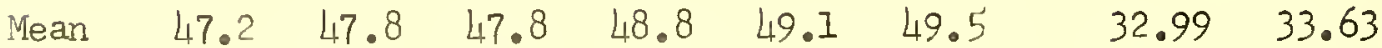


DIAMOND SHOALS LIGHTSHIP $35^{\circ} 05^{\prime} 30^{\prime \prime N}$. $75^{\circ} 19^{\prime} 30^{\prime \prime} \mathrm{W}$. Depth of water -200 feet

February, 1956

Temperature OF

Salinity $\%$

Depth

Day $0^{\prime} \quad 30^{\prime} \quad 50^{\prime} 100^{\prime} 150^{\prime} 200^{\prime} \quad 0^{\prime} 200^{\prime}$

$\begin{array}{lllllll}1 & 66.0 & 66.1 & 66.1 & 66.0 & 62.7 & 61.5\end{array}$

$\begin{array}{lllllll}2 & 60.8 & 60.7 & 60.7 & 60.7 & 60.2 & 58.1\end{array}$

$\begin{array}{lllllll}3 & 47.7 & 55.5 & 61.9 & 62.5 & 62.9 & 62.9\end{array}$

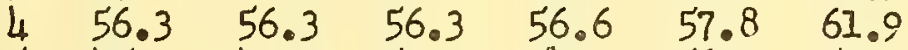

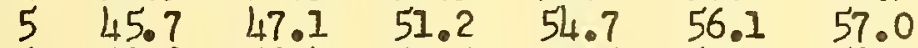

$\begin{array}{lllllll}6 & 68.8 & 68.4 & 67.6 & 61.6 & 56.9 & 58.0\end{array}$

$\begin{array}{lllllll}7 & 50.8 & 57.0 & 62.0 & 62.6 & 61.6 & 61.2\end{array}$

$\begin{array}{lllllll}8 & 67.5 & 67.4 & 67.4 & 65.0 & 62.3 & 62.3\end{array}$

$\begin{array}{lllllll}9 & 60.1 & 66.0 & 66.1 & 66.1 & 65.1 & 64.9\end{array}$

$\begin{array}{lllllll}10 & 64.1 & 64.3 & 65.2 & 65.1 & 64.7 & 63.8\end{array}$

$\begin{array}{lllllll}11 & 71.3 & 71.3 & 71.3 & 69.9 & 55.7 & 55.5\end{array}$

$\begin{array}{lllllll}12 & 46.2 & 50.2 & 64.1 & 66.9 & 61.8 & 58.6\end{array}$

$\begin{array}{lllllll}13 & 43.9 & 43.9 & 44.2 & 46.1 & 52.5 & 58.1\end{array}$

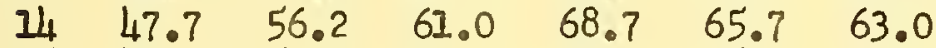

$\begin{array}{lllllll}15 & 45.8 & 57.9 & 63.7 & 68.0 & 65.1 & 66.0\end{array}$

$\begin{array}{lllllll}16 & 64.1 & 64.1 & 64.2 & 64.6 & 67.0 & 67.5\end{array}$

$\begin{array}{lllllll}17 & 50.0 & 61.1 & 61.9 & 66.2 & 66.7 & 66.8\end{array}$

$\begin{array}{lllllll}18 & 66.9 & 66.9 & 66.9 & 67.2 & 67.2 & 66.9\end{array}$

$\begin{array}{lllllll}19 & 69.2 & 69.1 & 69.1 & 67.8 & 66.4 & 66.0\end{array}$

$\begin{array}{lllllll}20 & 62.9 & 63.0 & 63.7 & 65.1 & 65.7 & 66.5\end{array}$

$\begin{array}{lllllll}21 & 44.9 & 43.8 & 50.0 & 64.4 & 65.7 & 66.5\end{array}$

$\begin{array}{lllllll}22 & 73.1 & 73.2 & 73.1 & 72.8 & 65.6 & 66.1\end{array}$

$\begin{array}{lllllll}23 & 71.6 & 71.7 & 71.7 & 71.4 & 70.1 & 66.8\end{array}$

$\begin{array}{lllllll}24 & 70.1 & 70.2 & 70.3 & 70.0 & 66.7 & 65.1\end{array}$

$\begin{array}{lllllll}25 & 56.2 & 63.7 & 67.0 & 66.5 & 62.0 & 62.2\end{array}$

$\begin{array}{lllllll}26 & 57.9 & 58.0 & 58.0 & 58.1 & 63.0 & 64.0\end{array}$

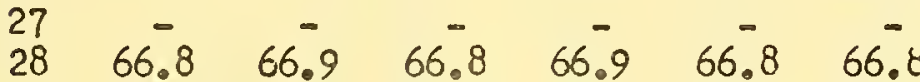
$\begin{array}{lllllll}29 & 57.1 & 57.1 & 57.4 & 60.1 & 61.1 & 62.0\end{array}$

30

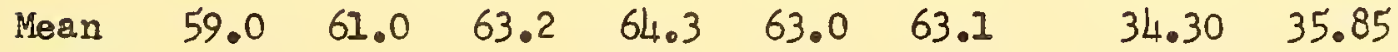


DIAMOND SHOAIS LIGHTSHIP $35^{\circ} 05^{\prime} 30^{\prime \prime}$. $75^{\circ} 19^{1} 30^{\prime \prime}$ W. Depth of water - 200 feet

March 1956

Temperature ${ }^{\circ} \mathrm{F}$

Salinity $\%$

Depth

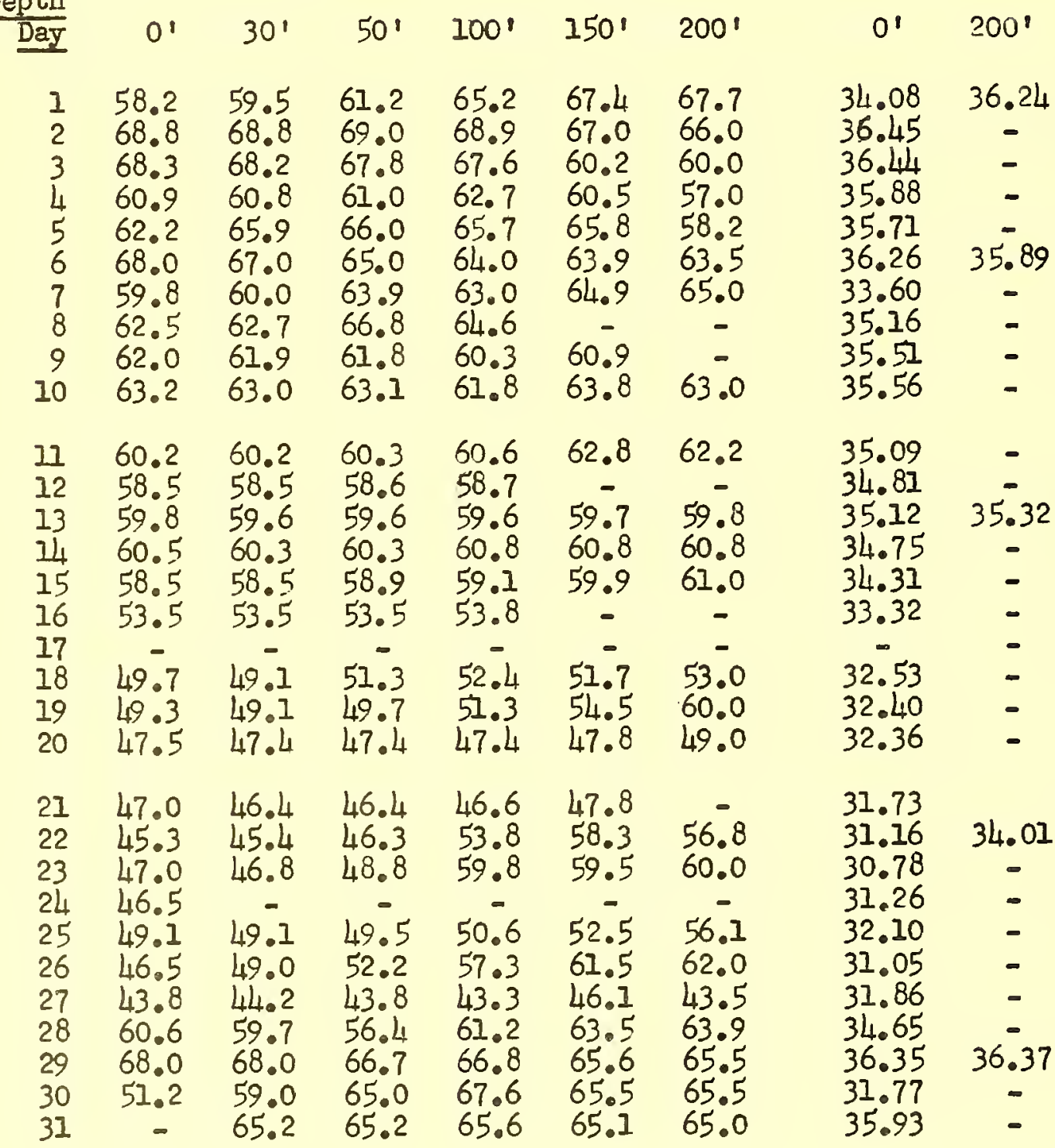

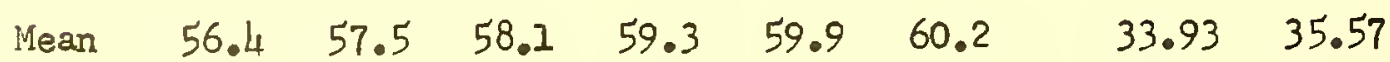


DIAMOND SHOALS LIGHTSHIP $35^{\circ} 05^{8} 30^{\prime \prime N}$. $75^{\circ} 19^{3} 30^{\prime \prime}$. Depth of water - 200 feet

\section{April 1956}

Temperature ${ }^{\circ} \mathrm{F}$

Salinity $\%$

Depth

Day $0^{\prime} \quad 30^{\prime} 50^{\prime} 100^{\prime} 150^{\prime} 200^{\prime} \quad 0^{\prime} 200^{\prime}$

$\begin{array}{lllllll}1 & 66.2 & 66.0 & 66.2 & 64.2 & 63.5 & 63.3\end{array}$

$\begin{array}{lllllll}2 & 71.3 & 66.2 & 64.2 & 67.1 & 66.0 & 65.8\end{array}$

$\begin{array}{lllllll}3 & 68.3 & 68.6 & 68.3 & 65.7 & 65.2 & 65.1\end{array}$

$\begin{array}{lllllll}4 & 55 . & 55.5 & 61.2 & 65.8 & 65.1 & 64.2\end{array}$

$\begin{array}{llllll}5 & 60.0 & 57.9 & 58.2 & 65.2 & 65.1\end{array}=$

$\begin{array}{lllllll}6 & 58.2 & 58.3 & 61.8 & 66.5 & 67.2 & 64.8\end{array}$

$\begin{array}{lllllll}7 & 60.1 & 59.7 & 59.9 & 65.9 & 66.2 & 64.5\end{array}$

$\begin{array}{lllllll}8 & 52.9 & 53.4 & 55.1 & 57.9 & 60.9 & 64.0\end{array}$

$\begin{array}{lllllll}9 & 57.1 & 61.3 & 64.9 & 68.6 & 65.4 & 64.4\end{array}$

$\begin{array}{lllllll}10 & 57.9 & 60.7 & 66.2 & 68.9 & 67.1 & 67.0\end{array}$

$\begin{array}{lllllll}11 & 58.2 & 58.3 & 58.6 & 61.9 & 63.3 & 64.0\end{array}$

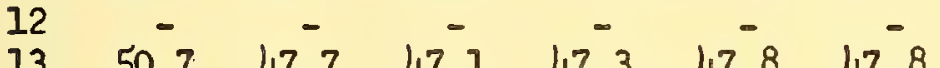

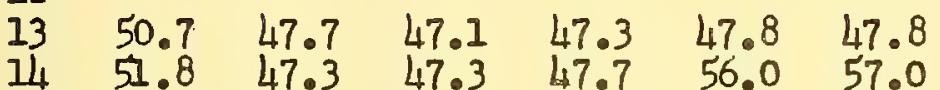

$\begin{array}{lllllll}15 & 70.7 & 70.7 & 70.4 & 71.1 & 69.3 & 68.9\end{array}$

16

17

$18 \quad 63.2 \quad 62.2 \quad 64.8$

$1970.60 .1 \quad 69.9$

- - -

$\begin{array}{lllllll}20 & 70.6 & 70.7 & 70.6 & 69.1 & 67.3 & 65.1\end{array}$

$\begin{array}{lllllll}21 & 61.8 & 61.5 & 61.4 & 62.1 & 62.0 & 65 .\end{array}$

$22-0-07.0-$

$\begin{array}{lllllll}23 & 62.1 & 67.1 & 67.9 & 67.8 & 67.8 & 67.8\end{array}$

$\begin{array}{lllllll}24 & 69.3 & 69.4 & 69.3 & 69.0 & 67.9 & 67.5\end{array}$

$\begin{array}{lllllll}25 & 69.1 & 69.3 & 69.3 & 67.9 & 67.9 & 67.4\end{array}$

$\begin{array}{lllllll}26 & 69.1 & 69.0 & 68.9 & 67.6 & 66.1 & 66.2\end{array}$

$\begin{array}{lllllll}27 & 69.8 & 68.8 & 68.7 & 67.1 & 65.3 & 64.0\end{array}$

$\begin{array}{lllllll}28 & 68.2 & 68.1 & 68.1 & 67.9 & 65.9 & 63.9\end{array}$

$\begin{array}{lllllll}29 & 68.3 & 68.4 & 68.1 & 67.9 & 65.2 & 64.8\end{array}$

$\begin{array}{lllllll}30 & 69.7 & 68.9 & 69.0 & 68.4 & 63.2 & 63.0\end{array}$

31

Mean

$\begin{array}{llllll}63.4 & 63.3 & 64.0 & 65.2 & 64.7 & 62.0\end{array}$

$\begin{array}{cc}36.14 & 35.67 \\ 36.20 & - \\ 32.21 & = \\ 33.51 & - \\ 32.80 & = \\ 33.48 & = \\ 32.28 & - \\ 33.57 & 35.75 \\ 33.50 & -\end{array}$

$34.19-$

$31.69=$

30.17 -

$36.05=$

- -

$34.31 \quad 36.01$

$36.13=$

36.58 -

$35.60=$

$35.54 \quad 36.25$

36.28 -

$36.33=$

$36.30=$

$36.31=$

33.25

36.29

$33.57 \quad 36.19$

$34.45 \quad 35.97$ 
DIAMOND SHOALS LIGHTSHIP $35^{\circ} 05^{\prime} 30^{\prime \prime} \mathrm{N} .7^{\circ} 19^{\prime} 30^{\prime \prime} \mathrm{W}$. Depth of water 200 feet

\section{May 1956}

$$
\text { Teniperature OF }
$$

Salinity $\%$

Depth

Day $0^{\prime} \quad 30^{\prime} 50^{\prime} 100^{\prime} 150^{\prime} 200^{\prime}$

$\begin{array}{lllllll}1 & 69.0 & 69.9 & 69.2 & 66.6 & 66.5 & 63.4\end{array}$

$\begin{array}{lllllll}2 & 72.8 & 71.7 & 70.9 & 68.1 & 66.2 & 66.1\end{array}$

$\begin{array}{lllllll}3 & 68.0 & 70.9 & 70.2 & 69.3 & 67.5 & 56.1\end{array}$

$\begin{array}{lllllll}4 & 57.4 & 62.8 & 66.2 & 67.0 & 67.0 & 65.1\end{array}$

$\begin{array}{lllllll}5 & 63.1 & 64.1 & 63.6 & 54.5 & 63.7 & 67.0\end{array}$

$\begin{array}{llcllll}6 & 64.9 & - & - & - & - & - \\ 7 & 57.0 & 53.8 & - & 65.9 & 66.2 & 64.0\end{array}$

$\begin{array}{ccccccc}7 & 57.0 & 53.8 & - & 65.9 & 66.2 & 64.0 \\ 8 & - & - & - & - & - & \end{array}$

9 53. - 50 - -50 -

$1060.0 \quad-\quad 63.1 \quad 70.0 \quad 67.3 \quad 66.6$

$\begin{array}{lllllll}11 & 58.8 & 57.5 & 53.7 & 68.6 & 65.0 & 63.1\end{array}$

$\begin{array}{llllll}12 & 62.7 & 57.4 & 56.4 & 56.5 & 63.7\end{array}$

$\begin{array}{lllllll}13 & 60.2 & 63.0 & 68.0 & 68.7 & 67.9 & 67.0\end{array}$

$14 \quad 64.5 \quad 64.2 \quad 63.4$

$\begin{array}{lll}15 & 65.7 & 65.7\end{array}$

61.7

68.2

67.9

64.8

67.2

67.2

73.070 .0

68.368 .3

74.0

70.9

68.2

$\begin{array}{lllll}17 & 76.5 & 76.7 & 76.2 & 740 \\ 18 & 75.6 & 74.8 & 74.1 & 70 \\ 19 & 81.0 & 81.5 & 81.3 & 7 \\ 20 & 76.0 & 77.8 & 77.7 & 70 .\end{array}$

70.0

68.7

68.2

$\begin{array}{lllll}17 & 76.5 & 76.7 & 76.2 & 74.0 \\ 18 & 75.6 & 74.8 & 74.1 & 70.0 \\ 19 & 81.0 & 81.5 & 81.3 & 77.3 \\ 20 & 76.0 & 77.8 & 77.7 & 70.5\end{array}$

70.6

69.2

$\begin{array}{lllll}17 & 76.5 & 76.7 & 76.2 & 740 \\ 18 & 75.6 & 74.8 & 74.1 & 70 \\ 19 & 81.0 & 81.5 & 81.3 & 7 \\ 20 & 76.0 & 77.8 & 77.7 & 70 .\end{array}$

$\begin{array}{lllll}17 & 76.5 & 76.7 & 76.2 & 740 \\ 18 & 75.6 & 74.8 & 74.1 & 70 \\ 19 & 81.0 & 81.5 & 81.3 & 7 \\ 20 & 76.0 & 77.8 & 77.7 & 70 .\end{array}$

$\begin{array}{lllll}17 & 76.5 & 76.7 & 76.2 & 740 \\ 18 & 75.6 & 74.8 & 74.1 & 70 \\ 19 & 81.0 & 81.5 & 81.3 & 7 \\ 20 & 76.0 & 77.8 & 77.7 & 70 .\end{array}$

70.5

$69.8 \quad 67.2$

21

22

2

2

2

\section{6}

\section{7}

\section{2}

29

30

$\begin{array}{lllllll}30 & 77.0 & 77.5 & 77.6 & 75.7 & 71.0 & 66.8\end{array}$

$\begin{array}{lllllll}31 & 73.3 & 73.0 & - & 71.3 & 66.0 & 62.8\end{array}$

68.968 .2

$68.0 \quad 67.1$

65.2

75.075 .4

76.3

71.2

66.5

$-$

78.7

78.8

77.2

78.9

$-$

$-$

75.175 .2

75.

68.

$-$

64.8

65.2
73.0
71.0
66.0

66.7

Mean

69.970 .4

70.7

69.0

67.6

66.3

$34.27 \quad 36.07$

$31.71=$

31.26 -

$\begin{array}{ll}31.81 & - \\ 31.82 & 36.00\end{array}$

32.79

35.56

36.11

36.06

36.07

36.07

$36.14 \quad 35.89$

$36.15=$

$35.69=$

36.10 -

36.08 -

$\begin{array}{ll}36.27 & - \\ 36.14 & 36.31\end{array}$

$36.15=$

$36.08=$ 
DIAMOND SHOAI LIGHTSHIP $35^{\circ} 05^{\prime} 30^{\prime \prime N}$. $75^{\circ} 19^{1} 30^{\prime \prime} \mathrm{W}$. Depth of water - 200 feet June 1956

Temperature ${ }^{\circ} \mathrm{F}$

Salinity $\%$

$\frac{\text { Depth }}{\text { Day }} \quad 0^{\prime} \quad 30^{\prime} \quad 50^{\prime} \quad 100^{\prime} 150^{\prime} 200^{\prime} \quad 0^{\prime} 200^{\prime}$

$\begin{array}{rrrrrrrrr}1 & - & - & - & - & - & - & - & - \\ 2 & 79 . & - & - & - & - & - & 36.08 & - \\ 3 & 77.6 & 77.0 & 77.0 & 76.3 & 71.5 & 64.2 & 36.17 & - \\ 4 & 77.8 & 77.3 & 77.0 & 76.5 & 71.6 & 64.6 & .17 & - \\ 5 & 76.7 & 76.3 & 76.0 & 74.1 & 67.5 & - & .06 & = \\ 6 & 75.8 & 75.7 & 75.7 & 71.3 & - & - & 36.11 & - \\ 7 & 75.8 & 75.8 & 75.5 & 75.0 & - & - & 35.91 & - \\ 8 & 73.0 & 73.2 & 73.4 & 74.8 & 74.3 & 65.6 & .44 & 36.23 \\ 9 & 79.0 & 77.2 & 76.2 & 75.0 & 69.8 & 65.8 & 35.92 & - \\ 10 & 67.5 & - & - & - & - & - & 33.13 & -\end{array}$

$\begin{array}{llllllllllll}11 & 67.0 & 67.0 & 66.1 & 68.3 & 72.9 & - & 29.64 & \text { - }\end{array}$

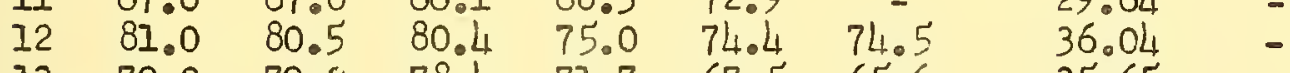

$\begin{array}{llllllll}13 & 79.0 & 79.0 & 78.4 & 71.7 & 67.5 & 65.6 & 35.65\end{array}$

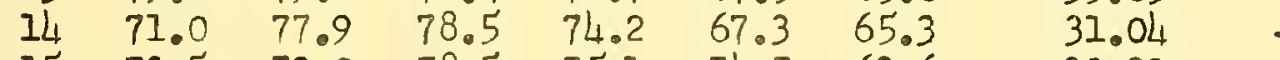

$\begin{array}{lllllllll}15 & 73.5 & 79.0 & 78.5 & 75.1 & 74.7 & 63.6 & 32.29 & - \\ 16 & 77.5 & 77.7 & 77.5 & 76.6 & 71.5 & 65.6 & 34.02 & 36.0\end{array}$

$\begin{array}{lllllllll}17 & 80.1 & 78.0 & 77.5 & 73.4 & 68.0 & 67.3 & 36.20 & -\end{array}$

$\begin{array}{llcccccc}18 & 79.0 & - & - & - & - & - & .09 \\ 19 & 78.0 & 77.8 & 77.7 & 77.5 & 77.3 & - & 36.17\end{array}$

$\begin{array}{llllllll}20 & 70.3 & 70.2 & 72.9 & 74.0 & 74.6 & 68.0 & -\end{array}$

$\begin{array}{lllllllll}21 & 71.3 & 74.8 & 75.8 & 76.3 & 69.3 \quad & - & 32.88 & -\end{array}$

$\begin{array}{lllllllll}22 & 75.3 & 74.0 & 74.9 & 75.6 & 69.4 & 66.2 & 34.69 & -\end{array}$

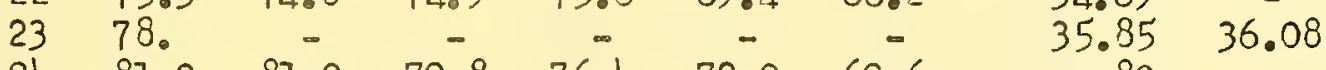

$\begin{array}{llllllll}24 & 81.0 & 81.0 & 79.8 & 76.4 & 72.0 & 69.6 & .80\end{array}$

$\begin{array}{llllllll}25 & 79.0 & 77.7 & 78.3 & 77.3 & 73.7 & 70.4 & 35.70\end{array}$

$\begin{array}{llllllll}26 & 82.4 & 80.7 & 79.3 & 73.3 & 69.0 & 67.3 & 36.05\end{array}$

$\begin{array}{lllllllll}27 & 80.5 & 80.3 & 77.7 & 68.6 & 65.5 & 63.8 & 36.14 & -\end{array}$

$\begin{array}{lllllllll}28 & 77.7 & 77.3 & 76.2 & 70.0 & 65.4 & 62.9 & 35.86 & - \\ 29 & 79.6 & 79.7 & 79.6 & 77.7 & 67.2 & 64.0 & .86 & =\end{array}$

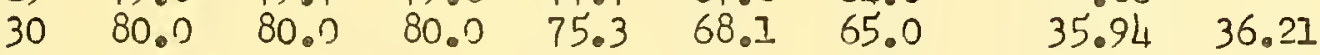

31

$\begin{array}{lllllllll}\text { Mean } & 76.5 & 77.0 & 75.8 & 74.4 & 70.6 & 66.3 & 35.10 & 36.15\end{array}$ 
DIAIOND SHOAL LIGHTSHIP $35^{\circ} 05^{\prime} 30^{\prime \prime N}$. $75^{\circ} 19^{\prime} 30^{\prime \prime}$. Depth of water - 200 feet

$$
\text { July } 1956
$$

$$
\text { Temperature oF }
$$

Salinity $\%$

Depth

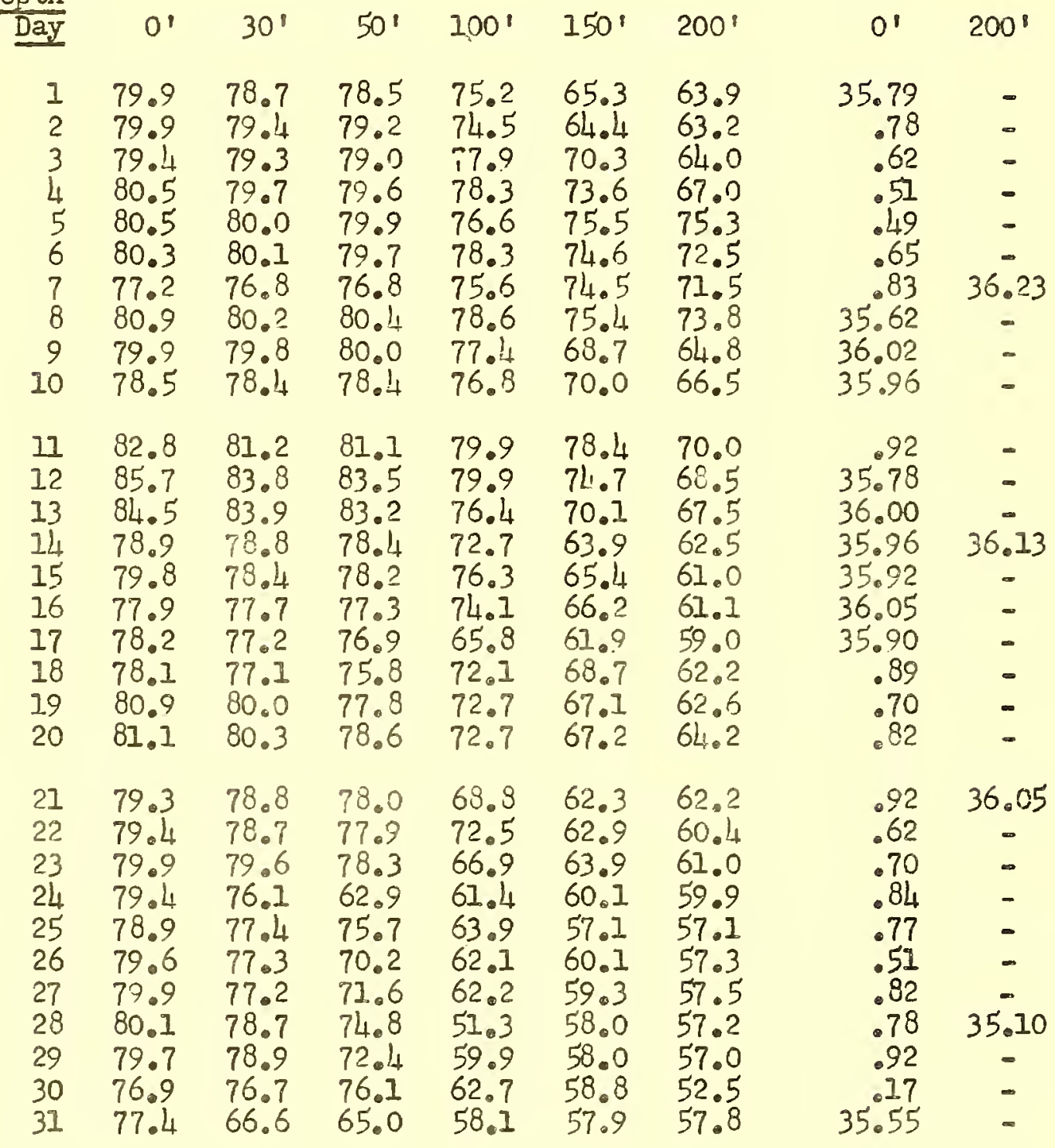

$\begin{array}{lllllllll}\text { Mean } & 79.9 & 78.6 & 76.9 & 71.0 & 66.3 & 63.3 & 35.77 & 35.88\end{array}$ 
DIAMOND SHOALS LIGHTSHIP $35^{\circ} 05^{\prime} 30^{\prime \prime} \mathrm{N} .75^{\circ} 19^{1} 30^{\prime \prime} \mathrm{W}$. Depth of water -200 feet August 1956

$$
\text { Temperature }{ }^{\circ} \mathrm{F}
$$

Depth
Salinity $\%$

$0^{\prime} \quad 200^{\prime}$

170

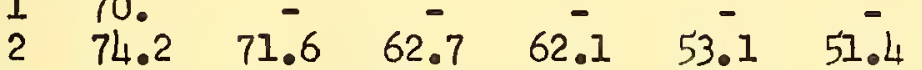

$\begin{array}{lllllll}3 & 74.7 & 72.6 & 66.7 & 60.7 & 60.1 & 57.5\end{array}$

$\begin{array}{lllllll}4 & 75.2 & 74.2 & 66.2 & 59.3 & 55.4 & 58.5\end{array}$

$\begin{array}{lllllll}5 & 75.2 & 73.9 & 68.9 & 59.8 & 57.1 & 55.0\end{array}$

$\begin{array}{lllllll}6 & 73.8 & 71.8 & 70.1 & 64.7 & 51.9 & 52.7\end{array}$

$\begin{array}{lllllll}7 & 75.8 & 74.8 & 74.7 & 64.2 & 60.7 & 54.5\end{array}$

$\begin{array}{lllllll}8 & 75.9 & 73.1 & 63.7 & 60.9 & 60.1 & 57.1\end{array}$

$\begin{array}{lllllll}9 & 76.2 & 71.8 & 65.2 & 62.0 & 56.8 & 49.6\end{array}$

$\begin{array}{lllllll}10 & 77.4 & 70.7 & 62.8 & 58.9 & 57.1 & 55.5\end{array}$

$\begin{array}{lllllll}11 & 78.8 & 71.7 & 69.6 & 61.2 & 54.0 & 52.0\end{array}$

$\begin{array}{lllllll}12 & 81.7 & 82.1 & 81.2 & 73.9 & 54.2 & 52.5\end{array}$

$\begin{array}{lllllll}13 & 78.3 & 80.8 & 78.7 & 72.3 & 61.9 & 52.0\end{array}$

$\begin{array}{lllllll}14 & 76.5 & 76.9 & 76.8 & 70.1 & 54.0 & 53.9\end{array}$

$\begin{array}{lllllll}15 & 77.6 & 76.2 & 74.3 & 73.6 & 67.1 & 59.1\end{array}$

$\begin{array}{lllllll}16 & 76.6 & 82.4 & 82.6 & 72.7 & 59.6 & 60.0\end{array}$

$\begin{array}{lllllll}17 & 76.3 & 75.3 & 71.6 & 70.0 & 66.7 & 58.6\end{array}$

$\begin{array}{lllllll}18 & 77.9 & 81.1 & 80.8 & 68.3 & 64.7 & 58.0\end{array}$

$\begin{array}{lllllll}19 & 77.3 & 79.4 & 75.8 & 68.9 & 61.1 & 60.2\end{array}$

$\begin{array}{lllllll}20 & 80.3 & 79.7 & 80.0 & 76.0 & 65.2 & 61.7\end{array}$

$\begin{array}{lllllll}21 & 78.9 & 83.3 & 82.3 & 78.1 & 66.9 & 61.6\end{array}$

$\begin{array}{lllllll}22 & 74.1 & 71.7 & 69.2 & 65.3 & 62.4 & 61.2\end{array}$

$\begin{array}{lllllll}23 & 75.6 & 75.1 & 74.9 & 69.9 & 59.7 & 59.1\end{array}$

$\begin{array}{lllllll}24 & 75.8 & 74.1 & 73.9 & 69.6 & 59.9 & 56.1\end{array}$

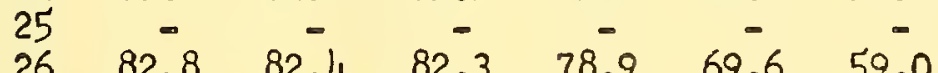

27

28

29

30

31

77.6

82.4

82.3

78.9

73.7

75.6

67.0

60.7

60.960 .1

$62.4 \quad 59.5$

$63.2 \quad 58.0$

81.0

77.1

78.7

77.6

72.5

$68.7 \quad 61.0$

Mean

77.

83.9

$83.1 \quad 79.9$

\begin{abstract}
79.9
\end{abstract}
$60.7-57.1$

$\begin{array}{cc}30.09 & - \\ 31.47 & - \\ 33.23 & 34.48 \\ 32.31 & - \\ 31.46 & - \\ 36.68 & - \\ 31.86 & - \\ 31.21 & - \\ 31.53 & =\end{array}$

$\begin{array}{cc}30.93 & 33.49 \\ 32.47 & - \\ 31.66 & - \\ 31.26 & - \\ 31.71 & - \\ 32.14 & - \\ 32.57 & - \\ 31.55 & 34.51 \\ 31.86 & - \\ 33.30 & -\end{array}$

32.84

32.30

31.19

30.98

$35.81 \quad 36.30$

35.85 -

32.73

32.08

32.46

33.86

$32.39 \quad 34.70$ 
DIAMOND SHOAL IIGHTSHIP $35^{\circ} 05^{\prime} 30^{\prime \prime} \mathrm{N} .75^{\circ} 19^{1} 30^{\prime \prime} \mathrm{W}$. Depth of water - 200 feet

September 1956

Tenperature OF

Salinity $\%$

Depth

Day $0^{\prime} \quad 30^{\prime} 50^{\prime} 100^{\prime} 150^{\prime} 230^{\prime}$

$0^{\prime} \quad 200^{\prime}$

$\begin{array}{lllllll}1 & 81.0 & 80.8 & 80.5 & 80.1 & 77.2 & 62.5\end{array}$

$33.13 \quad 35.61$

$284.3 \quad 84.2$

$83.7 \quad 80.5$

74.4

62.4

$\begin{array}{llll}83.3 & 82.1 & 81.9 & 75.4\end{array}$

65.060 .0

$4 \quad 81.5 \quad 80.7$

$80.0 \quad 78.1$

$77.7 \quad 69.0$

$84.8 \quad 83.8$

82.378 .2

71.064 .0

$84.0 \quad 83.3$

$76.3 \quad 67.0$

65.061 .4

$80.9 \quad 77.2 \quad 71.0 \quad 62.2 \quad 61.0 \quad 60.1$

8

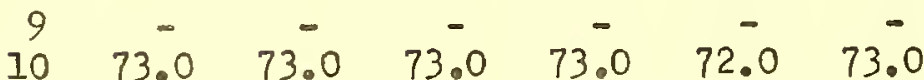

$\begin{array}{lllllll}11 & 73.7 & 72.4 & 71.4 & 71.8 & 72.2 & 72.2\end{array}$

$\begin{array}{lllllll}12 & 74.6 & 73.8 & 72.9 & 73.0 & 73.3 & 64.5\end{array}$

$\begin{array}{lllllll}13 & 74.0 & 73.5 & 72.7 & 76.2 & 66.0 & 63.8\end{array}$

$\begin{array}{lllllll}14 & 75.5 & 75.9 & 78.6 & 82.5 & 81.0 & 69.0\end{array}$

15

79.5

$81.0 \quad 76.3$

66.9

61.8

60.0

16

$76.4 \quad 76.8 \quad 76.0$

77.3

63.560 .1

77.0

78.7

78.9

77.8

71.3

62.0

18

19

20

$\begin{array}{lll}76.0 & 76.7 & 76.4\end{array}$

$78.2 \quad 67.9$

61.3

$69.0 \quad 61.9$

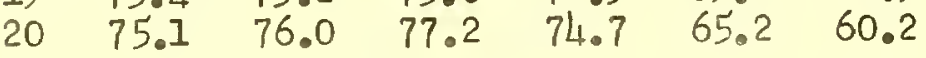

$\begin{array}{lllllll}21 & 73.3 & 73.0 & 73.0 & 74.0 & 74.8 & 71.5\end{array}$

22

23

24

24

2

26

27

28

29

30

74.3

82.0

74.3

81.8

74.5

80.4

74.

79.4

77.7

77.0

68.0

73.0

76.5

77.5

69.5

77.3

69.5

75.0

69.1

69.3

$68.4 \quad 70.0 \quad 70.8$

68.8

$\begin{array}{llll}68.1 & 67.1 & 69.0 & 73.8\end{array}$

68.9

69.5

68.7

31

$\begin{array}{lllllll}\text { Mean } & 76.6 & 76.5 & 76.0 & 74.1 & 70.7 & 66.5\end{array}$

$33.30 \quad 34.31$ 
DIAMOND SHOALS LIGHTSHIP $35^{\circ} 05^{1} 30^{\prime \prime N}$. $75^{\circ} 29130^{\prime \prime}$. Depth of water - 200 feet

\title{
October 1956
}

\author{
Temperature ${ }^{\circ} \mathrm{F}$
}

Salinity $\%$

Depth

Day $0^{\prime} \quad 30^{\prime} \quad 50^{\prime} \quad 100^{\prime} 150^{\prime} 200^{\prime}$

$\begin{array}{lllllll}1 & 70.7 & 69.7 & 71.6 & 68.2 & 75.0 & 75.5\end{array}$

$\begin{array}{lllllll}2 & 71.5 & 71.3 & 71.1 & 72.1 & 75.7 & 75.5\end{array}$

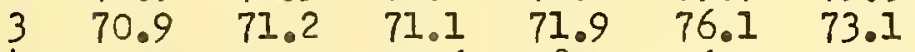

$\begin{array}{lllllll}4 & 71.9 & 70.9 & 71.6 & 78.3 & 76.9 & 73.1\end{array}$

$\begin{array}{lllllll}5 & 71.1 & 70.9 & 71.8 & 75.9 & 76.0 & 70.1\end{array}$

$\begin{array}{lllllll}6 & 72.0 & 71.9 & 72.3 & 75.1 & 76.7 & 68.0\end{array}$

$\begin{array}{lllllll}7 & 71.2 & 71.2 & 76.2 & 78.2 & 74.2 & 67.6\end{array}$

$\begin{array}{lllllll}8 & 71.2 & 71.1 & 70.9 & 74.0 & 75.9 & 71.6\end{array}$

$\begin{array}{lllllll}9 & 72.3 & 71.0 & 71.4 & 75.8 & 72.1 & 62.4\end{array}$

10

$\begin{array}{lllllll}11 & 69.0 & 68.9 & 69.0 & 69.6 & 70.4 & 74.8\end{array}$

$\begin{array}{lllllll}12 & 68.8 & 68.8 & 69.2 & 74.7 & 77.8 & 75.2\end{array}$

$\begin{array}{lllllll}13 & 67.4 & 67.2 & 67.1 & 67.1 & 74.7 & 76.5\end{array}$

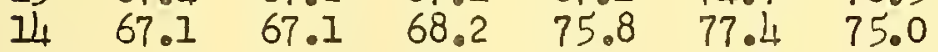

$\begin{array}{lllllll}15 & 73.9 & 75.2 & 77.1 & 78.4 & 75.1 & 72.3\end{array}$

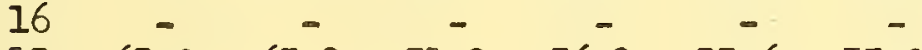

$\begin{array}{lllllll}17 & 67.0 & 67.3 & 71.9 & 76.3 & 77.6 & 77.0\end{array}$

$\begin{array}{lllllll}18 & 73.9 & 74.4 & 75.6 & 77.9 & 76.1 & 72.4\end{array}$

19 - $\quad$ - $\quad$ - $\quad$ - $\quad$ -

$\begin{array}{lllll}21 & 65.9 & 65.9 & 67.4 & 75.4\end{array}$

$\begin{array}{lllll}22 & 67.1 & 78.2 & 78.3 & 78.3\end{array}$

$\begin{array}{lllllll}23 & 67.2 & 67.8 & 67.9 & 77.9 & 77.1 & 67.5\end{array}$

$\begin{array}{lllllll}24 & 66.9 & 69.3 & 70.7 & 76.6 & 77.1 & 70.0\end{array}$

$25=-$ - - - -

$\begin{array}{lcccccc}26 & - & - & - & - & - & -\end{array}$

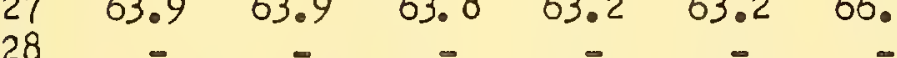

$\begin{array}{lllllll}29 & 63.9 & 63.8 & 63.8 & 63.3 & 63.1 & -\end{array}$

$\begin{array}{lllllll}30 & 63.9 & 63.9 & 63.4 & 63.2 & 63.1 & 68.0\end{array}$

$\begin{array}{lllllll}31 & 65.1 & 63.9 & 63.8 & 63.9 & 76.9 & -\end{array}$

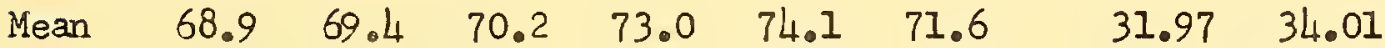


DIA 10 ND SHOALS LIGHTSHP $35^{\circ} 05^{\prime} 30^{\prime \prime N} .75^{\circ} 19^{\prime} 30^{\prime \prime}$. D. Depth of water - 200 feet

November 1956

Temperature oF

Salinity $\%$

Depth

Day $0^{\prime} \quad 30^{\prime} 50^{\prime} 100^{\circ} 150^{\prime} 200^{\prime} \quad 0^{\prime} 200^{\prime}$

$\begin{array}{ccccccccc}1 & 66.0 & 65.2 & 65.0 & 64.7 & 74.0 & 76.0 & 31.51 & - \\ 2 & 67.0 & 57.0 & 67.2 & 69.5 & 73.7 & 75.9 & 31.47 & - \\ 3 & 68.5 & 69.2 & 75.0 & 78.2 & 77.6 & 79.2 & 32.74 & - \\ 4 & 65.1 & 65.1 & 65.0 & 66.8 & 77.0 & 77.5 & 31.63 & 36.24 \\ 5 & 63.9 & 63.9 & 63.8 & 66.5 & 73.6 & 77.0 & .22 & - \\ 6 & 63.8 & 63.5 & 63.3 & 63.4 & 62.8 & 69.0 & .36 & - \\ 7 & 64.0 & - & - & - & - & - & 31.52 & - \\ 8 & 63.0 & 75.8 & 77.6 & 77.8 & 77.1 & 76.0 & 30.92 & - \\ 9 & - & - & - & - & - & - & - & - \\ 10 & 64.0 & 65.3 & 67.3 & 73.2 & 73.9 & 73.4 & 32.22 & 36.01\end{array}$

$\begin{array}{llllllllll}17 & 65.0 & 76.5 & 76.4 & 76.2 & 75.7 & 74.1 & 30.92 & \text { - }\end{array}$

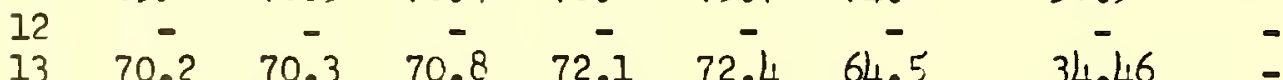

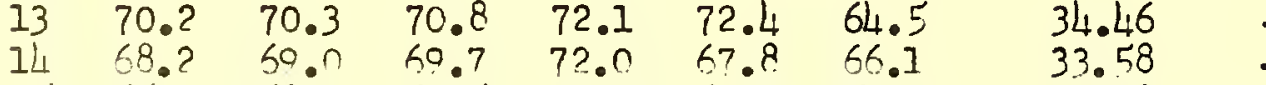

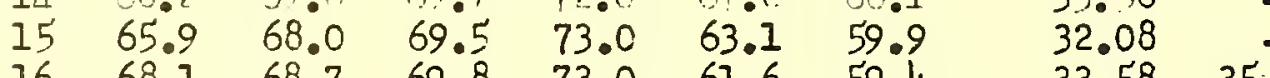

$\begin{array}{lllllllll}16 & 68.1 & 68.7 & 69.8 & 73.0 & 61.6 & 59.4 & 33.58 & 35.92 \\ 17 & 67.8 & 68.5 & 69.3 & 69.8 & 62.5 & 58.5 & 32.99 & \end{array}$

$\begin{array}{lllllllll}17 & 67.8 & 68.5 & 69.3 & 69.8 & 62.5 & 58.5 & 32.99 & -\end{array}$

$\begin{array}{ccccccccc}18 & 67.1 & 67.1 & 67.3 & 69.0 & 69.1 & 69.0 & 33.85 & - \\ 20 & 67 . & - & - & - & - & - & 33.47 & -\end{array}$

$\begin{array}{llllllll}21 & 67.2 & 67.2 & 68.1 & 75.9 & 71.7 & 71.6 & 33.76\end{array}$

$\begin{array}{llllllll}22 & 71.1 & 71.0 & 71.0 & 71.9 & 74.0 & - & 34.87\end{array}$

$\begin{array}{lllllllll}23 & 76.3 & 76.2 & 76.1 & 75.8 & 75.1 & - & 36.21 & - \\ 24 & 74.0 & 74.0 & 74.0 & 74.0 & 73.5 & 71.3 & \end{array}$

$\begin{array}{lllllll}25 & 73.5 & 73.3 & 73.2 & 72.9 & 72.1 & 69.8\end{array}$

$\begin{array}{lllllll}26 & 64.0 & 64.4 & 65.6 & 71.0 & 71.5 & 66.9\end{array}$

$\begin{array}{lllllll}27 & 64.6 & 64.6 & 64.6 & 67.5 & 69.5 & 69.9\end{array}$

$\begin{array}{lllllll}28 & 59.0 & 59.0 & 60.9 & 68.5 & 69.1 & 69.1\end{array}$

$\begin{array}{lllllll}29 & 59.6 & 59.7 & 62.1 & 66.3 & 69.9 & 64.8\end{array}$

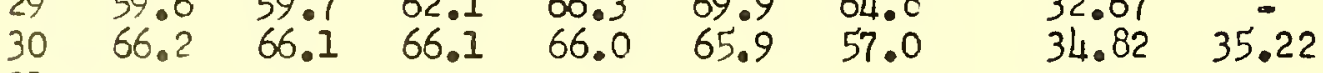

31

$\begin{array}{lllllll}\text { Mean } & 66.7 & 67.9 & 68.7 & 71.0 & 71.0 & 69.4\end{array}$

$33.14 \quad 35.92$ 
Table 15 (cont'd)

DIAMOND SHOALS LIGHTSHIP $35^{\circ} 05^{1} 30^{\prime \prime N} \mathrm{~N} .75^{\circ} 19^{1} 30^{\prime \prime}$. Depth of water -200 feet

\section{December 1956}

Temperature ${ }^{\circ} \mathrm{F}$

Depth

Day

270.3

365.5

59.8

66.8

59.6

60.3

65.2

9
10 60.6

1168.3

1263.8

1361.0

14 68.8

$15 \quad 63.6$

65.0

$17 \quad 59.3$

$18 \quad 59.6$

$19 \quad 59.2$

$20 \quad 61.0$

2171.9

2260.8

2364.2

$24 \quad 60.0$

$25 \quad 58.2$

$26 \quad 57.0$

$27 \quad 59.3$

$28 \quad 61.3$

2962.1

30

31

$30^{\prime}$

$50^{\circ}$

$70.2 \quad 69.9$

65.5

66.1

61.2

60.2

65.8

65.0

62.5

69.2

69.1

68.8

71.0

60.6

60.6

68.5

65.3

68.4

60.6

70.3

61.3

69.0

68.8

63.5

65.3

65.2

59.2

59.1

59.5

59.6

59.1

59.2

59.8

67.7

60.8

71.9

59.9

67.3

59.4

59.359 .2

61.3

62.7 $100^{\prime}$

$150^{\prime} 200^{\circ}$

62.3

62.0

66.3

62.7

61.8

62.7

61.0

59.0

63.2

63.9

63.7

69.4

63.2

62.9

66.5

61.3

$-$

62.4

66.9

63.3

64.1

69.2

63.0

61.7

60.4

60.6

59.6

68.6

63.4

62.3

64.2

62.3

63.1

63.9

62.0

63.0

60.6

64.8

58.0

61.3

67.6

61.3

58.9

62.9

63.1

62.2

62.5

61.9

62.9

63.6

63.8

64.0

63.5

64.2

$65.0 \quad 62.9$

71.5

65.0

63.5

$68.5 \quad 67.4 \quad 66.0$

$60.7 \quad 67.8 \quad 66.9$

62.8

62.7

66.5

58.3

63.0

65.2

62.8

64.3

65.2

62.8

65.0

64.4

62.5

Mean

62.7

63.1

64.0

64.4

$63.6 \quad 62.8$
$33.48 \quad(35.95)$

Salinity $\%$

$0^{\prime} 200^{\prime}$

$32.64=$

$35.33=$

$34.33=$

$34.84=$

$33.29=$

$35.46=$

$\begin{array}{cc}34.16 & - \\ 32.94 & - \\ 34.15 & 3.95 \\ 33.54 & - \\ 32.65 & - \\ 32.04 & = \\ -0 & - \\ 32.82 & - \\ 33.13 & -\end{array}$

$\begin{array}{cc}32.68 & = \\ 33.72 & - \\ 33.09 & = \\ 32.42 & - \\ 31.91 & = \\ 32.40 & = \\ = & - \\ - & - \\ - & -\end{array}$
$34.82=$ 
Surface temperatures tended to lag below the mean for the period of record (1947-1956) during the first half of the year except for mid-February. The latter two-thirds of November tended well below the mean and the latter two-thirds of December tended well above.

Minimum temperatures of less than $59^{\circ} \mathrm{F}$ were observed in January. Maximum at the surface, reater than $81^{\circ} \mathrm{F}$ was reached in late August and early September, at the bottom, $81{ }^{\circ} \mathrm{F}$ at the same time. The thermocline did not become as well developed at this location as it did north of Cape Hatteras. The maximum gradient, surface to bottom (70 feet) was never more than $9^{\circ} \mathrm{F}$. The thernocline comenced development in late April, yet during the first-third of June the water column was again virtually isothermal. The strong thermocline during the latter part of July was apparently due to a transgression of colder water from offshore.

The surface and bottom salinities seldom differed by more than $1 \%$ and remained very close to $36 \%$ oxcept during November when the salinity of the whole water column dropped to about $34 \%$. This was coupled wi th a depression in the temperature of the whole water column, followed by a return to higher salinity and temperature in December. It appears that two unusual phenomena were involved here at nearly the same time. First there was a transgression of less saline water followed by colder than normal weather causing a depression in water temperatures. The extremely warm air temperatures enjoyed by the south eastern seaboard were reflected in the rise in water temperature in December.

It is difficult to believe that the depression in salinity throughout the whole water column was due to the off-shore movement of coastal water, as a relatively shailow volume of such water when mixed with a deeper, larger volume in the proportions available would not produce such a marked salinity depression. In consideration of the wind systems prevalent at the time, we suspect this to be a parcel of coastal water from north of Cape Hatteras carried southwesterly by a Iong period of predominantiy northerly and northeasterly winds. The speed of movement of this $p$ recel from Hatteras to the lightship may have been on the order of 7 - 8 miles per day. This phenomenon has previously been postulated by Bumpus and Pierce (1955, pp. 95 and 96). 

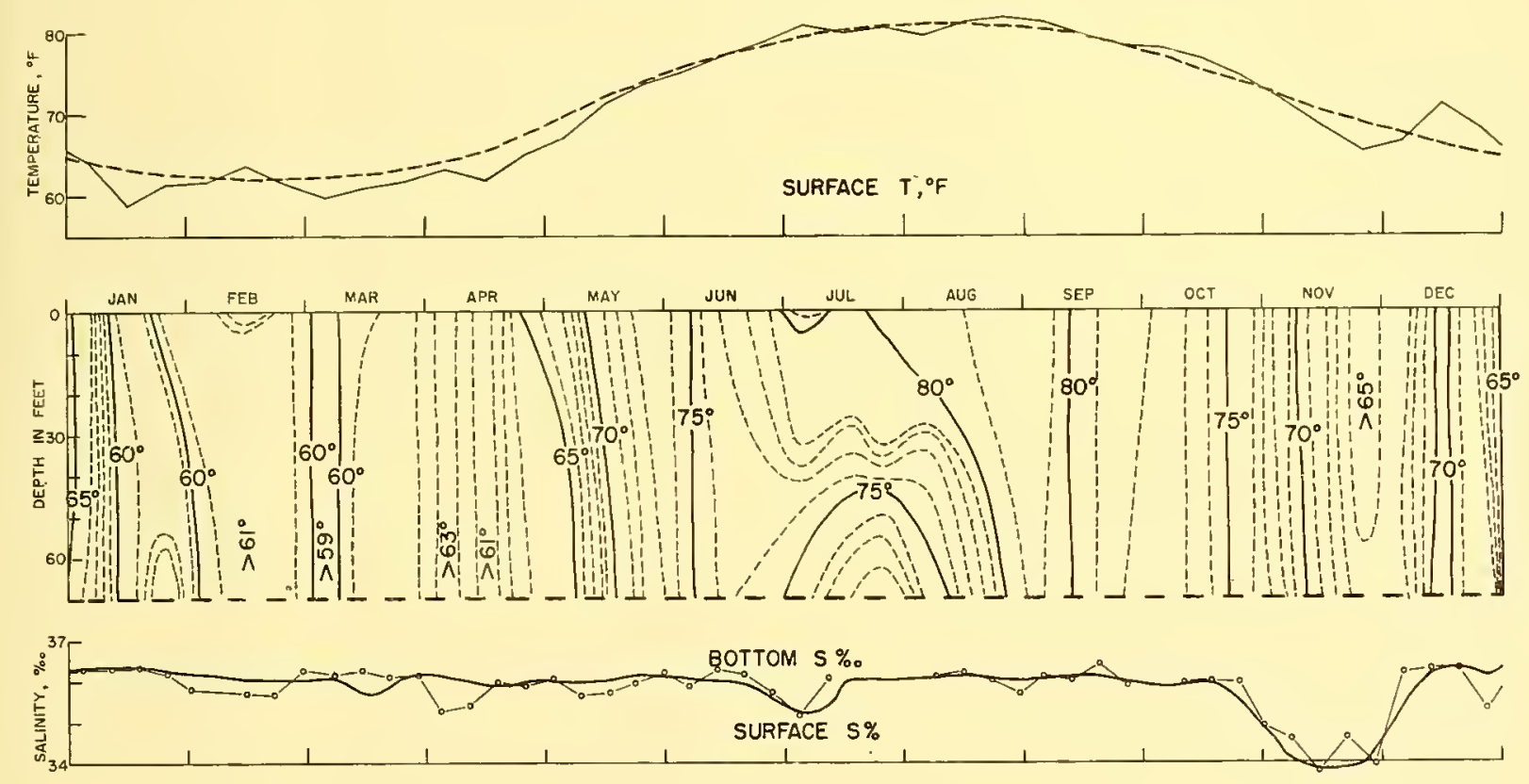

Migure 17. Frying Pan Shoals Lightship, 1956. (Dashed line in upper diagram, mean for period 1947-1956) 


\section{5}

\section{November}

Temperature ${ }^{\circ}$ Salinity $\%$

$\frac{\text { Depth }}{\text { Day }} \quad 0^{\prime} \quad 30^{\prime} 50^{\prime} 70^{\prime} \quad 0^{\prime} \quad 70^{\prime}$

1

2

3

4

5

6

?

9

10

11

12

13

14

15

16

17

18

19

20

21

22

23

24

25

26

27

28

29

$\begin{array}{lllllll}30 & 63.2 & 63.0 & 63.0 & 63.0 & 35.88 & 35.85\end{array}$

31

Mean
December

Temperature ${ }^{\circ} \mathrm{F} \quad$ Salinity $\%$

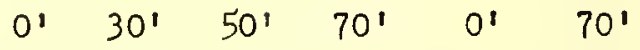

$\begin{array}{llllll}62.2 & 62.2 & 62.2 & 62.2 & 35.88 & -\end{array}$

$\begin{array}{llllll}69.0 & 67.6 & 67.1 & 66.9 & 36.27 & -\end{array}$

$\begin{array}{llllll}73.1 & 73.1 & 73.1 & 73.1 & .24 & -\end{array}$

$\begin{array}{llllll}73.9 & 73.7 & 73.7 & 73.7 & .24 & -\end{array}$

$74.4 \quad 74.1 \quad 74.0 \quad 74.0 \quad .19 \quad-$

$\begin{array}{lllllc}73.5 & 73.5 & 73.4 & 73.3 & .20 & - \\ 73.9 & 73.6 & 73.6 & 73.0 & .12 & 36.14\end{array}$

$\begin{array}{lllll}75.1 & 75.1 & 70.9 & 66.6 & .10\end{array}$

$\begin{array}{lllll}74.6 & 74.6 & 72.3 & 66.3 & .07\end{array}$

$\begin{array}{llllll}67.2 & 67.2 & 67.1 & 67.1 & .06 & -\end{array}$

$\begin{array}{llllll}68.8 & 68.8 & 67.2 & 65.9 & .20 & -\end{array}$

$\begin{array}{lllll}64.9 & 64.7 & 64.7 & 64.6 & .31\end{array}$

$\begin{array}{llllll}62.6 & 62.5 & 62.5 & 62.6 & .08 & -\end{array}$

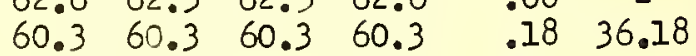

$\begin{array}{llllll}70.3 & 70.2 & 62.0 & 60.9 & .19 & \text { - }\end{array}$

$\begin{array}{llllll}63.4 & 63.6 & 63.6 & 63.6 & .21 & -\end{array}$

$\begin{array}{llllll}66.9 & 66.7 & 66.4 & 65.9 & .24 & -\end{array}$

$\begin{array}{llllll}69.3 & 69.1 & 67.9 & 67.4 & .24 & -\end{array}$

$\begin{array}{llllll}66.2 & 66.2 & 66.2 & 66.2 & .26 & -\end{array}$

$\begin{array}{lllll}66.3 & 66.3 & 66.3 & 66.3 & .28\end{array}$

$\begin{array}{llllll}72.7 & 72.6 & 72.6 & 68.3 & .10 & 36.23\end{array}$

$\begin{array}{llllll}70.7 & 70.7 & 70.5 & 70.5 & .16 & -\end{array}$

$\begin{array}{llllll}71.7 & 71.7 & 71.7 & 71.7 & .13 & -\end{array}$

$\begin{array}{llllll}70.3 & 70.3 & 70.3 & 70.3 & .18 & -\end{array}$

$\begin{array}{llllll}68.5 & 68.3 & 68.3 & 68.3 & .26 & -\end{array}$

$\begin{array}{llllll}64.7 & 64.7 & 64.7 & 64.7 & .19 & -\end{array}$

$\begin{array}{llllll}65.6 & 65.6 & 65.5 & 65.5 & .24 & -\end{array}$

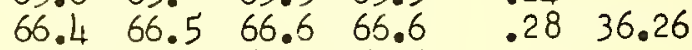

$\begin{array}{llllll}60.1 & 60.1 & 59.6 & 59.6 & .31 & -\end{array}$

$\begin{array}{llllll}61.1 & 61.0 & 60.9 & 60.9 & .33 & -\end{array}$

$\begin{array}{lllll}63.1 & 63.1 & 62.7 & 62.0 & .26\end{array}$

$\begin{array}{llllll}68.0 & 68.0 & 67.3 & 66.7 & 36.19 & 36.20\end{array}$ 
FRYING PAN SHOALS LIGHTSHIP $33^{\circ} 28^{\prime} 00^{\prime \prime N} \cdot 77^{\circ} 33^{\prime} 08^{\prime \prime}$.

Depth of water - 72 feet

1956

$\underline{\text { January }}$

February

Temperature ${ }^{\circ} \mathrm{F}$

Salinity $\%$

Temperature ${ }^{\circ} \mathrm{F}$

Salinity $\%$

Depth

Day $0^{\prime} 30^{\prime} 50^{\prime} 70^{\prime} \quad 0^{\prime} 70^{\prime}$

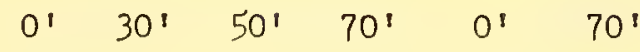

$\begin{array}{llllll}1 & 66.0 & 68.1 & 68.0 & 68.0 & 36.26\end{array}$

$\begin{array}{lllllll}2 & 66.9 & 66.8 & 66.8 & 66.7 & .32 & - \\ 3 & 66.0 & 65.9 & 66.0 & 66.0 & .32 & -\end{array}$

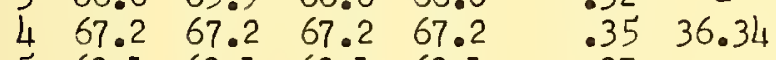

$\begin{array}{lllll}5 & 63.1 & 63.1 & 63.1 & 63.1\end{array}$

$\begin{array}{lllll}6 & 60.2 & 58.8 & 58.3 & 58.3\end{array}$

$.37 \quad-$

$\begin{array}{lllll}7 & 68.8 & 66.4 & 63.2 & 60.9\end{array}$

$\begin{array}{lllll}8 & 67.5 & 67.5 & 67.4 & 67.4\end{array}$

$\begin{array}{llllll}9 & 55.8 & 58.2 & 58.2 & 58.2 & .37\end{array}$

$\begin{array}{llllll}10 & 62.4 & 64.1 & 64.2 & 63.2 & .34\end{array}$

$11 \quad 63.0 \quad 63.0 \quad 63.0 \quad 63.1 \quad 31 \quad .36 .30$

$\begin{array}{lllll}12 & 64.9 & 64.9 & 64.9 & 64.9\end{array}$

$\begin{array}{lllll}13 & 60.9 & 61.9 & 62.0 & 62.0\end{array}$

$\begin{array}{lllll}14 & 61.9 & 61.9 & 61.9 & 61.9\end{array}$

$\begin{array}{lllll}15 & 57.0 & 58.7 & 57.7 & 57.6\end{array}$

$\begin{array}{lllll}16 & 60.3 & 60.2 & 60.1 & 60.1\end{array}$

$\begin{array}{lllll}17 & 56.9 & 58.1 & 58.1 & 58.0\end{array}$

$\begin{array}{lllll}18 & 60.0 & 59.9 & 59.8 & 59.8\end{array}$

$\begin{array}{lllllll}19 & 49.1 & 50.0 & 51.1 & 52.9 & 35.63 & - \\ 20 & 53.8 & 53.7 & 53.3 & 52.9 & 36.35 & -\end{array}$

$.30-$

$.56 \quad-$

$.34 \quad-$

36

.42

36.46

$\begin{array}{llllll}55.2 & - & - & - & 35.85 & 35.84 \\ 64.0 & - & - & - & 36.14 & - \\ 54.5 & - & - & - & 36.65 & - \\ 55 . & - & - & - & 35.83 & - \\ 65.1 & - & - & - & 36.17 & - \\ 67.0 & - & - & - & .30 & - \\ 64.5 & - & - & - & .20 & - \\ 65 . & - & - & - & .16 & - \\ 63 . & - & - & - & .21 & - \\ 64 . & - & - & - & .29 & -\end{array}$

$2151.3 \quad 51.1 \quad 51.050 .5 \quad .17$ -

$\begin{array}{ccccccc}22 & - & - & - & - & .178 \\ 23 & 68.9 & 68.8 & 68.1 & 58.5 & .38 & -\end{array}$

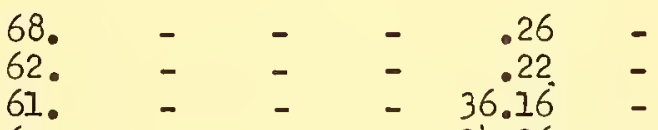

60. - - - 34.36

56. - - $\quad-35.64$

65. - - - $\quad-36.32$

35.71

63.

73. - - -

$63 . \quad-\quad-\quad-24$

65.

$-\quad 36.35$

$\begin{array}{lccccc}60 . & - & - & - & 35.60 & - \\ 59.8 & 60.0 & 60.0 & 60.0 & .69 & 35.69\end{array}$

$\begin{array}{lllllll}59.0 & 59.0 & 59.1 & 59.0 & 35.42 & -\end{array}$

$\begin{array}{lllll}24 & 61.2 & 61.2 & 61.2 & 61.2\end{array}$

$25 \quad 57.8 \quad 54.8 \quad 54.0 \quad 54.1$

$\begin{array}{lllll}26 & 56.8 & 56.8 & 56.7 & 56.7\end{array}$

27

$\begin{array}{llllll}28 & 65 . & - & - & - & - \\ 29 & 64 . & - & - & - & -\end{array}$

3064.5 - - -

.220.

$\begin{array}{lllll}62.8 & 62.8 & 62.8 & 62.8 & 36.16\end{array}$

$\begin{array}{llll}67.2 & 67.2 & 67.2 & 67.2\end{array}$

$61.9 \quad 62.0 \quad 62.0 \quad 62.1$

.47

$\begin{array}{lllll}61.7 & 61.7 & 61.8 & 61.8 & .30\end{array}$

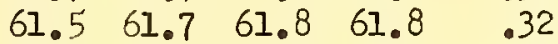

$.32-$

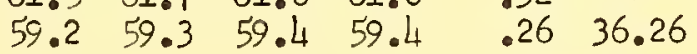

$3163 . \quad-\quad-\quad .31$

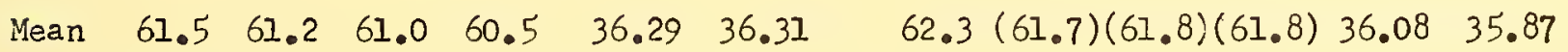


FRYING PAN SHOAIS LIGHTSHIP

March

Temperature ${ }^{\circ} \mathrm{F}$ $33^{\circ} 28^{\prime} 00^{\prime \prime N} \cdot 77^{\circ} 33^{\prime} 08^{\prime \prime W}$. 1956
Depth of water - 72 feet

\begin{tabular}{|c|c|c|c|c|c|c|c|c|c|c|c|c|}
\hline Day & $0^{\prime}$ & $30^{\prime}$ & $50^{\prime}$ & $70^{\prime}$ & $0^{1}$ & $70^{\prime}$ & $0^{\prime}$ & $30^{\prime}$ & $50^{\prime}$ & $70^{\prime}$ & $0^{\prime}$ & $70^{\prime}$ \\
\hline $\begin{array}{l}1 \\
2 \\
3 \\
4 \\
5 \\
6 \\
7 \\
8 \\
9 \\
10\end{array}$ & $\begin{array}{l}57.0 \\
57.0 \\
59.2 \\
60.5 \\
58.8 \\
60.7 \\
60.5 \\
62.8 \\
59.9 \\
60.0\end{array}$ & $\begin{array}{l}56.8 \\
57.1 \\
58.8 \\
60.5 \\
58.0 \\
60.4 \\
60.5 \\
62.8 \\
60.0 \\
60.0\end{array}$ & $\begin{array}{l}56.8 \\
57.2 \\
58.6 \\
60.5 \\
59.0 \\
60.3 \\
60.5 \\
62.9 \\
60.0 \\
60.0\end{array}$ & $\begin{array}{c}56.8 \\
57.2 \\
58.6 \\
60.5 \\
59.0 \\
- \\
60.5 \\
62.9 \\
60.0 \\
-\end{array}$ & $\begin{array}{r}35.96 \\
- \\
36.22 \\
.21 \\
.36 \\
.20 \\
.14 \\
36.29 \\
35.79 \\
35.69\end{array}$ & $\begin{array}{l}- \\
- \\
- \\
- \\
- \\
- \\
36.14 \\
- \\
- \\
-\end{array}$ & $\begin{array}{l}64.2 \\
66.7 \\
66.6 \\
60.5 \\
64.0 \\
61.0 \\
63.8 \\
62.5 \\
61.5 \\
61.7\end{array}$ & $\begin{array}{l}64.1 \\
66.2 \\
66.6 \\
60.9 \\
64.0 \\
61.3 \\
63.9 \\
62.7 \\
61.5 \\
61.6\end{array}$ & $\begin{array}{l}64.0 \\
66.1 \\
66.5 \\
60.9 \\
64.0 \\
62.2 \\
63.9 \\
62.7 \\
61.5 \\
61.6\end{array}$ & $\begin{array}{l}63.9 \\
66.1 \\
66.5 \\
60.9 \\
64.0 \\
62.6 \\
63.9 \\
62.7 \\
61.5 \\
61.6\end{array}$ & $\begin{array}{r}36.21 \\
.49 \\
.37 \\
- \\
36.00 \\
35.52 \\
36.12 \\
.10 \\
36.06 \\
35.88\end{array}$ & $\begin{array}{c}- \\
- \\
- \\
35.27 \\
- \\
- \\
- \\
- \\
- \\
-\end{array}$ \\
\hline $\begin{array}{l}11 \\
12 \\
13 \\
14 \\
15 \\
16 \\
17 \\
18 \\
19 \\
20\end{array}$ & $\begin{array}{l}61.0 \\
60.2 \\
63.1 \\
64.2 \\
64.2 \\
60.7 \\
59.9 \\
57.2 \\
59.9 \\
57.5\end{array}$ & $\begin{array}{l}61.9 \\
62.0 \\
63.1 \\
64.2 \\
64.2 \\
60.1 \\
60.0 \\
57.2 \\
59.9 \\
57.6\end{array}$ & $\begin{array}{l}62.2 \\
62.3 \\
63.1 \\
64.2 \\
64.2 \\
59.5 \\
60.0 \\
57.4 \\
60.0 \\
57.5\end{array}$ & $\begin{array}{l}62.3 \\
62.4 \\
63.1 \\
64.2 \\
64.2 \\
59.0 \\
60.0 \\
60.0 \\
60.0 \\
57.5\end{array}$ & $\begin{array}{r}36.24 \\
34.50 \\
36.15 \\
.24 \\
.33 \\
35.16 \\
.53 \\
.03 \\
.70 \\
35.30\end{array}$ & $\begin{array}{c}- \\
- \\
- \\
36.26 \\
- \\
- \\
- \\
- \\
- \\
-\end{array}$ & $\begin{array}{l}60.0 \\
60.1 \\
62.8 \\
63.0 \\
62.5 \\
62.7 \\
62.5 \\
62.2 \\
61.3 \\
61.9\end{array}$ & $\begin{array}{l}60.0 \\
60.2 \\
62.3 \\
62.8 \\
62.5 \\
62.6 \\
62.5 \\
62.1 \\
61.4 \\
61.9\end{array}$ & $\begin{array}{l}60.1 \\
60.2 \\
62.3 \\
62.9 \\
62.5 \\
62.5 \\
62.6 \\
62.1 \\
61.4 \\
61.9\end{array}$ & $\begin{array}{l}60.1 \\
60.2 \\
62.5 \\
62.9 \\
62.6 \\
62.7 \\
62.7 \\
62.1 \\
61.5 \\
61.9\end{array}$ & $\begin{array}{l}.41 \\
.66 \\
.70 \\
.88 \\
.98 \\
.97 \\
.96 \\
.82 \\
.97 \\
.97\end{array}$ & $\begin{array}{c}35.40 \\
- \\
- \\
- \\
= \\
- \\
= \\
35.98 \\
- \\
-\end{array}$ \\
\hline $\begin{array}{l}21 \\
22 \\
23 \\
24 \\
25 \\
26 \\
27 \\
28 \\
29 \\
30 \\
31\end{array}$ & $\begin{array}{l}62.4 \\
59.7 \\
50.3 \\
61.3 \\
59.8 \\
59.8 \\
58.6 \\
61.2 \\
68.4 \\
64.8 \\
62.0\end{array}$ & $\begin{array}{l}62.5 \\
59.5 \\
50.1 \\
61.3 \\
59.9 \\
59.9 \\
59.0 \\
61.2 \\
68.3 \\
61.9 \\
62.0\end{array}$ & $\begin{array}{l}62.5 \\
59.5 \\
60.1 \\
61.3 \\
59.9 \\
59.9 \\
59.0 \\
51.2 \\
62.0 \\
64.9 \\
62.0\end{array}$ & $\begin{array}{l}62.5 \\
59.5 \\
60.1 \\
61.3 \\
60.0 \\
59.9 \\
59.0 \\
61.2 \\
60.7 \\
64.9 \\
62.0\end{array}$ & $\begin{array}{r}36.17 \\
.02 \\
.07 \\
.09 \\
.16 \\
36.12 \\
35.88 \\
36.15 \\
.22 \\
.28 \\
.20\end{array}$ & $\begin{array}{c}36.11 . \\
- \\
- \\
- \\
- \\
- \\
36.14 \\
- \\
- \\
-\end{array}$ & $\begin{array}{l}61.5 \\
61.5 \\
62.3 \\
61.8 \\
61.9 \\
70.2 \\
69.8 \\
66.9 \\
67.5 \\
66.8\end{array}$ & $\begin{array}{l}61.7 \\
61.5 \\
62.3 \\
61.9 \\
62.0 \\
68.7 \\
67.9 \\
66.8 \\
66.6 \\
66.2\end{array}$ & $\begin{array}{l}61.7 \\
61.5 \\
62.3 \\
61.9 \\
62.0 \\
64.0 \\
67.5 \\
66.8 \\
66.3 \\
66.2\end{array}$ & $\begin{array}{l}61.7 \\
61.5 \\
62.3 \\
61.9 \\
62.0 \\
62.5 \\
67.4 \\
66.8 \\
66.3 \\
66.2\end{array}$ & $\begin{array}{r}.93 \\
- \\
.87 \\
.78 \\
35.77 \\
36.33 \\
.31 \\
.15 \\
36.18 \\
35.94\end{array}$ & $\begin{array}{c}- \\
- \\
- \\
- \\
35.85 \\
- \\
- \\
- \\
- \\
-\end{array}$ \\
\hline
\end{tabular}


FRYING PAN SHOALS LTGHTSHIP
Depth of water - 72 feet

$$
1956
$$

May

$$
\text { Temperature OF }
$$

Salinity $\%$

Temperature ${ }^{\circ} \mathrm{F}$

June

Day $0^{\prime} \quad 30^{\prime} \quad 50^{\prime} \quad 70^{\prime} \quad 0^{\prime} \quad 70^{\prime}$

$\begin{array}{llllll}1 & 64.2 & 63.8 & 63.7 & 63.7 & 36.07\end{array}$

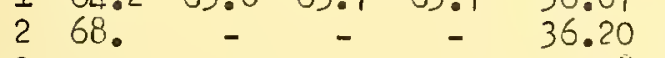

$\begin{array}{lcccccc}3 & - & - & - & - & .18 & -\end{array}$

$\begin{array}{lllllll}4 & 68.0 & 67.0 & 65.1 & 65.2 & .12 & - \\ 5 & 65.0 & 65.0 & 64.9 & 64.8 & .05 & -\end{array}$

$670 . \quad-\quad-\quad-$

768 .

$864.3 \quad 64.5 \quad 64.5 \quad 64.5$

.20

36.06

$9 \quad 63.4 \quad 63.5 \quad 63.4 \quad 63.4$

.65

$\begin{array}{llllll}10 & 71.9 & 66.3 & 65.4 & 65.4 & 36.13\end{array}$

$11 \quad 73.0 \quad 73.0 \quad 73.0 \quad 73.0$

$.17 \quad-$
$.10 \quad-$

$\begin{array}{lllll}12 & 70.0 & 69.2 & 69.1 & 69.0 \\ 13 & 69.2 & 69.2 & 69.2 & 69.2\end{array}$

$\begin{array}{lllll}14 & 70.5 & 70.7 & 70.7 & 70.7\end{array}$

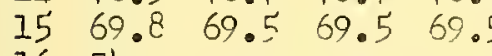

1674.

36.16

35.94

.78

$\begin{array}{lllll}17 & 68.2 & 67.8 & 67.5 & 67.5\end{array}$

.66

35.83

36.12

1971.5

$68.967 .0 \quad 67.0$

$\begin{array}{lll}70.3 & 68.9 & 68.9\end{array}$

35.95

20

$\begin{array}{lllll}21 & 70.2 & 69.4 & 67.8 & 67.9\end{array}$

$2270.5-\quad-\quad 36.22$

2378.

2474.

2573.5

2673.

2773.

$\begin{array}{ll}28 & 76 . \\ 29 & 71 .\end{array}$

3073.

3174.

(7)

$74.3 \quad 74 . \overline{1} 73.8$

.22

.06

.14

.60

$\begin{array}{lll}73.5 & 73.5 & 73.7\end{array}$

$\begin{array}{lll}73.5 & 73.5 & 73.5\end{array}$

.13

$\begin{array}{cccc}- & - & - & 36.16 \\ 71.2 & 71.2 & 71.2 & 35.86\end{array}$

73.573 .5

73.5

36.16

36.20

$-$

-

35.65

$0^{\prime} \quad 30^{\prime} 50^{\prime} 70^{\prime}$

$0^{\prime}$

$70^{\prime}$

$\begin{array}{lllll}74.7 & 74.3 & 74.1 & 74.0 & 36.00\end{array}$

$75 . \quad-\quad-\quad 35.92$

$\begin{array}{lllll}75.5 & 75.2 & 75.1 & 75.1 & 36.07\end{array}$

$\begin{array}{lllll}75.1 & 75.0 & 75.0 & 75.0 & 36.00\end{array}$

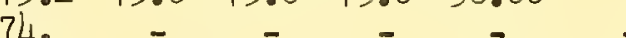

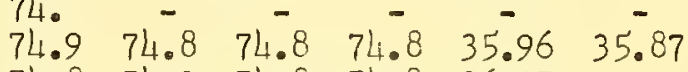

$\begin{array}{lllll}74.8 & 74.9 & 74.8 & 74.8 & 36.07\end{array}$

$\begin{array}{lllll}74.1 & 74.0 & 73.9 & 73.9 & 35.91\end{array}$

$\begin{array}{lllll}75.1 & 74.5 & 74.4 & 74.4 & 35.98\end{array}$

76.

$-\quad 36.13$

$\begin{array}{lccc}76.0 & - & - & - \\ 77.2 & 76.4 & 76.4 & 76.3\end{array}$

$\begin{array}{llll}77.5 & 77.2 & 77.1 & 77.1\end{array}$

77.2

$77.9 \quad 77.4 \quad 77.3$

$35^{\circ} .74$

78.0

77.

$\begin{array}{lllll}76.0 & 75.9 & 75.8 & 75.5 & 35.88\end{array}$

$77.977 .977 .9-36.1$

36.16

$\begin{array}{ccccc}77 . & - & - & - & .17 \\ 78.0 & 77.8 & 77.7 & 77.7 & .18\end{array}$

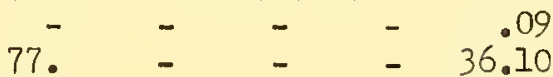

$78 . \quad-\quad-35.44$

$\begin{array}{cccccc}79.0 & 77.0 & - & - & .62 & - \\ 79.3 & 79.2 & 78.3 & 77.4 & .48 & 35.68\end{array}$

$\begin{array}{llll}79.3 & 77.8 & 75.4 & 75.2\end{array}$

.13

$79.576 .3 \quad 74.574 .4$

35.03

36.19

80.

$\begin{array}{llllll}76.9 & 76.2 & 75.8 & 75.5 & 35.86 & 36.00\end{array}$
77.276 .0 


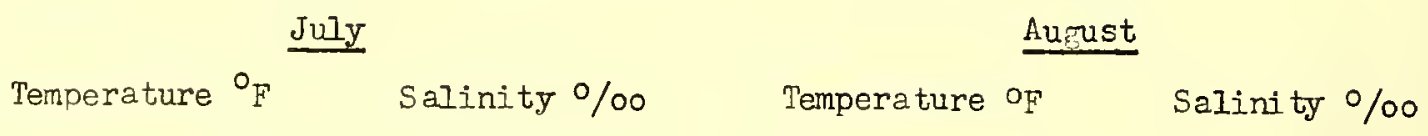

Depth $\quad 0^{\prime} \quad 30^{\prime} 50^{\prime} 70^{\prime} \quad 0^{\prime} 70^{\prime}$

$179.8 \quad 79.3 \quad 75.2 \quad 74.5 \quad 34.44=$

$\begin{array}{llllll}2 & 82.1 & 81.8 & 75.7 & 75.2 & 34.98\end{array}$

$\begin{array}{llllll}3 & 80.9 & 80.1 & 75.9 & 73.8 & 35.10\end{array}$

$\begin{array}{lllllll}4 & 80.7 & 80.5 & 75.9 & 73.9 & .12 & 35 .\end{array}$

$\begin{array}{llllll}5 & 81 . & - & - & - & .14 \\ 6 & 81 . & - & - & - & .46\end{array}$

$\begin{array}{llcccc}7 & 81.1 & 77.8 & 75.3 & 74.8 & .36 \\ 8 & 80 . & . & - & - & .36\end{array}$

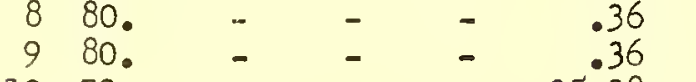

$1079 . \quad-\quad-\quad-35.28$

II 80. - $\quad-\quad-\quad 36.0836 .0$

$\begin{array}{lllllll}12 & 80.7 & 79.4 & 77.1 & 76.6 & 35.52 & -\end{array}$

$1380 . \quad$ - $\quad$ - $\quad$ - 35.56

I4 $80 . \quad-\quad-\quad-35.84$

$\begin{array}{llllll}15 & 78.2 & 77.0 & 74.5 & 71.0 & 36.06\end{array}$

$\begin{array}{llllll}16 & 77.7 & 76.3 & 73.2 & 71.0 & .06\end{array}$

$1780.0-16$ - $\quad-\quad .14$

$1879 . \quad-\quad-\quad-16.16$

$\begin{array}{llcccc}19 & 80 . & - & - & - & 36.14 \\ 20 & 80.9 & 78.2 & 72.7 & 72.4 & 35.95\end{array}$

$2180 . \quad-\quad-\quad \quad-\quad .86$

$\begin{array}{llllll}22 & 79.3 & 78.9 & 75.0 & - & 35.96\end{array}$

$\begin{array}{llllll}23 & 80.5 & 79.9 & 74.9 & 73.7 & 36.08\end{array}$

$\begin{array}{llllll}24 & 81.1 & 79.3 & 72.9 & 72.5 & .00\end{array}$

$\begin{array}{llllll}25 & 80.6 & 80.5 & 71.6 & 71.0 & .02\end{array}$

$\begin{array}{llllll}26 & 81.1 & 81.0 & 80.6 & - & .08\end{array}$

$\begin{array}{llllll}27 & 80.9 & 79.9 & 72.9 & 70.5 & 36.06\end{array}$

$\begin{array}{llllll}28 & 81.1 & 73.9 & 71.0 & 70.9 & 35.82\end{array}$

$\begin{array}{cccccc}29 & 79 . & - & - & - & .93 \\ 30 & 80.9 & 79.7 & 73.0 & - & .92\end{array}$

3180.0 - $-\quad$ - 35.95

$\begin{array}{llllll}\text { Mear: } \quad 80.2 & 79.0 & 74.6 & 73.0 & 35.70\end{array}$

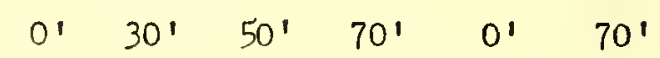

$\begin{array}{llllll}80.0 & 73.2 & 69.8 & 69.9 & 35.90 & 36.18\end{array}$

$\begin{array}{cccccc}80.6 & 80.1 & 75.5 & 72.9 & 35.76 & -\end{array}$

$\begin{array}{llllll}79.8 & 78.3 & 74.2 & 74.0 & 36.12 & -\end{array}$

$\begin{array}{lllll}79.3 & 79.0 & 74.1 & 73.9 & .10\end{array}$

$\begin{array}{llllll}77.9 & 77.9 & 77.9 & 77.9 & .26 & -\end{array}$

$\begin{array}{lllllll}78.1 & 78.0 & 78.0 & 78.0 & 36.11 & -\end{array}$

$\begin{array}{llllll}78.9 & 78.8 & 76.7 & 76.1 & 35.98 & 36.08\end{array}$

$\begin{array}{llllll}79.9 & 75.1 & 73.9 & 73.8 & 36.00 & -\end{array}$

$\begin{array}{llllll}79.8 & 76.4 & 76.1 & 76.0 & .13 & -\end{array}$

$\begin{array}{llllll}79.9 & 78.9 & 75.7 & 75.1 & .14 & -\end{array}$

$\begin{array}{llllll}79.9 & 78.8 & 77.1 & 75.0 & .14 & -\end{array}$

$\begin{array}{llllll}81.4 & 80.2 & 77.6 & 76.1 & .12 & -\end{array}$

$\begin{array}{llllll}80.8 & 80.3 & 77.8 & 76.0 & .04 & -\end{array}$

$\begin{array}{llllll}81.1 & 80.0 & 78.3 & 75.9 & 36.00 & 36.18\end{array}$

$\begin{array}{lllllll}80.8 & 80.3 & 78.0 & 78.0 & .02 & \text { - }\end{array}$

$\begin{array}{lllll}82.0 & 80.9 & 77.4 & 77.4 & .12\end{array}$

$\begin{array}{llllll}81.9 & 80.8 & 78.1 & 77.9 & .09 & -\end{array}$

$\begin{array}{rrrrrr}80.2 & 80.1 & 80.0 & 79.9 & .19 & - \\ 81.5 & 79.2 & 77.6 & 77.4 & 36.01 & -\end{array}$

$\begin{array}{lllll}80.8 & 80.2 & 76.3 & 76.2 & 35.78\end{array}$

$78.178 .178 .1=36.0736 .00$

$\begin{array}{llllll}78.9 & 79.0 & 79.0 & 79.0 & .12 & -\end{array}$

$\begin{array}{llllll}81.2 & 79.8 & 79.1 & 79.0 & 36.32 & -\end{array}$

$83.182 .380 .8-35.88-$

$83.082 .980 .2-.94-$

$\begin{array}{llllll}83.9 & 83.8 & 83.1 & 83.1 & .57 & -\end{array}$

$\begin{array}{llllll}83.2 & 83.1 & 82.4 & 82.1 & .95 & -\end{array}$

$\begin{array}{llllll}83.1 & 82.8 & 82.7 & 81.1 & .61 & 35.66\end{array}$

$\begin{array}{ccccccc}- & 81.9 & 81.5 & 79.9 & 79.5 & 35.95 & - \\ - & - & - & - & - & 36.12 & -\end{array}$ 
FRYING PA: SHOALS LIGHTSHIP $33^{\circ} 28^{\circ} 00^{\prime \prime N}, 77^{\circ} 33^{\prime} 08^{\prime \prime} \mathrm{W}$.

Depth of water - 72 feet

1956

September

Temperature ${ }^{\circ} \mathrm{F}$

Depth Day $0^{\prime} \quad 30^{\circ} \quad 50^{\prime} \quad 70^{\prime} \quad 0^{\prime} \quad 70^{\prime}$

$\begin{array}{lllllll}1 & 81.4 & 81.2 & 81.1 & 81.1 & 36.17\end{array}$

$\begin{array}{lllllll}2 & 81.0 & 80.9 & 80.8 & 80.7 & .04 & -\end{array}$

$\begin{array}{lllllll}3 & 81.2 & 81.0 & 81.0 & 81.0 & .11\end{array}$

$\begin{array}{llllll}4 & 81.8 & 81.4 & 81.3 & 81.2 & .10\end{array}$

$\begin{array}{lllllll}5 & 81.1 & 81.1 & 81.1 & 80.9 & .12 & 36.01\end{array}$

$\begin{array}{lllll}6 & 81.3 & 81.3 & 81.0 & 81.0\end{array}$

$\begin{array}{lllll}7 & 81.2 & 81.0 & 80.9 & 80.9\end{array}$

$\begin{array}{llll}8 & 81.5 & 81.5 & 81.5 \quad-\end{array}$

9

10

17

1

$\begin{array}{llll}80.1 & 80.0 & 80.0 & 79.9\end{array}$

$\begin{array}{llllll}13 & 79.9 & 79.7 & 79.6 & -\end{array}$

$\begin{array}{lllll}14 & 80.4 & 80.1 & 80.0 & 80.0\end{array}$

$\begin{array}{lllll}15 & 80.5 & 80.4 & 80.5 & 80.4\end{array}$

$\begin{array}{lllll}16 & 79.0 & 78.8 & 78.8 & 78.8\end{array}$

$\begin{array}{lllll}17 & 79.0 & 79.0 & 79.0 & 79.1\end{array}$

$\begin{array}{lllll}18 & 78.8 & 78.7 & 78.7 & -\end{array}$

$\begin{array}{lllll}19 & 78.5 & 78.5 & 78.6 & 78.6\end{array}$

$20 \quad 79.0 \quad 78.7 \quad 78.6 \quad 78.6$

$\begin{array}{lllll}21 & 78.5 & 78.5 & 78.5 & 78.5\end{array}$

$\begin{array}{lllll}22 & 78.2 & 78.1 & 78.1 & 78.0\end{array}$

23

$24790 \quad 78-9 \quad 78.8-$

$25 \quad 78.2 \quad 78.2 \quad 78.3 \quad 78.2$

$\begin{array}{lllll}26 & 78.6 & 78.5 & 78.5 & 78.5\end{array}$

$\begin{array}{lllll}27 & 78.5 & 78.5 & 78.4 & 78.3\end{array}$

$\begin{array}{llllll}28 & 78.1 & 77.6 & 77.3 & 77.2\end{array}$

$\begin{array}{llll}29 & 77.7 & 77.7 & 77.5\end{array}$

$\begin{array}{lllll}30 & 77.3 & 77.2 & 77.3 & 77.1\end{array}$

31
Salinity $\%$

.12

.02

.10

36.08

36.09

.02

.03

.07

.04

.13

.17

.18

$.18 \quad 36.38$

.18 -

.26

.15

.02

36.13

35.94

.87

.95

.83

35.91

36.13
$-$

$-$

$-$

$-$

$-$

36.00

$-$

-

$-$

$-$

$-$

$-$

$-$

-

$-$

$-$

35.86

-

$-$

$-$

-

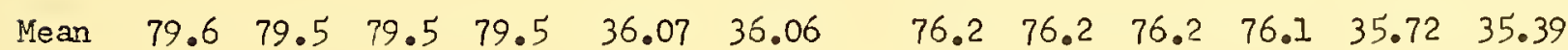
October

Temperature ${ }^{{ }_{F}}$

$0^{\prime} 30^{\prime} 50^{\prime} 70^{\prime}$

78.

$\begin{array}{llll}77.9 & 77.8 & 77.7 & 77.7\end{array}$

$\begin{array}{llll}78.1 & 77.8 & 77.8 & 77.8\end{array}$

$78.5 \quad 78.4 \quad 78.3 \quad 78.3$

$78.178 .178 .1 \quad 78.1$

$\begin{array}{lllll}78.4 & 78.4 & 78.4 & 78.4\end{array}$

$77.9 \quad 77.7 \quad 77.6 \quad 77.6$

$77.4 \quad 77.3 \quad 77.3 \quad 77.3$

$77.5 \quad 77.4 \quad 77.5 \quad 77.5$

$\begin{array}{llll}77.2 & 77.2 & 77.2 & 77.2\end{array}$

$\begin{array}{lllll}76.4 & 76.4 & 76.4 & 76.4 & 35.98\end{array}$

$\begin{array}{llllll}75.9 & 76.0 & 75.9 & 76.0 & 36.09\end{array}$

$\begin{array}{llll}75.6 & 75.8 & 75.7 & 75.7\end{array}$

$\begin{array}{llll}76.1 & 76.0 & 76.0 & 76.0\end{array}$

$\begin{array}{llll}77.3 & 77.2 & 77.2 & 77.0\end{array}$

$77.0 \quad 77.5 \quad 77.5 \quad 77.5$

$77.5 \quad 77.5 \quad 77.6 \quad 77.6$

$78.1 \quad 78.0 \quad 78.1 \quad 78.0$

$77.2 \quad 77.2 \quad 77.2 \quad 77.0$

$\begin{array}{llll}74.3 & 74.2 & 73.1 & 73.0\end{array}$

$\begin{array}{llll}76.0 & 75.8 & 75.5 & 75.0\end{array}$

$\begin{array}{llll}75.7 & 75.1 & 75.1 & 75.0\end{array}$

$\begin{array}{llll}76.7 & 76.7 & 76.7 & 76.5\end{array}$

$\begin{array}{llll}76.4 & 76.5 & 76.5 & 76.4\end{array}$

$75.9 \quad 75.9 \quad 75.9 \quad 75.9$

$72.3 \quad 72.3 \quad 72.4 \quad 72.4$

$\begin{array}{llll}72.9 & 72.9 & 72.9 & 72.9\end{array}$

$\begin{array}{ccccc}74 . & - & - & - & 35.61 \\ 73.4 & 73.4 & 73.5 & 73.5 & 34.06\end{array}$

$\begin{array}{llll}72.5 & 73.1 & 73.1 & 73.1\end{array}$

$\begin{array}{llll}72.0 & 72.1 & 72.3 & 72.5\end{array}$
Salinity $\%$

$0^{\prime} 70^{\prime}$

$\begin{array}{cc}35.95 & - \\ .94 & - \\ .78 & 34.28 \\ .91 & - \\ .80 & - \\ .80 & - \\ .81 & - \\ .83 & - \\ .92 & - \\ .91 & 35.90\end{array}$

$.10-$

$.07 \quad-$

36.02

$35.98 \quad 35.97$

$.99=$

82

$$
.69 \quad-
$$

.80

.78

.92

35.92

.69

.82

5.61
4.06

.64

$33.57 \quad 34.88$ 
FRYING PAN SHOALS LIGHTSHIP $\quad 33^{\circ} 28^{\prime} 00^{\prime \prime N} .77^{\circ} 33^{\prime} 08^{\prime \prime}$. $\quad$ Depth of water - 72 feet 1956

\author{
November \\ Temperature $\mathrm{OF} \quad$ Salinity $\%$
}

Depth

Day $0^{\prime} \quad 30^{\prime} \quad 0^{\prime} \quad 70^{\prime} \quad 0^{\prime} \quad 70^{\prime}$

$\begin{array}{lllll}1 & 72.2 & 72.3 & 72.6 & 72.6\end{array}$

$\begin{array}{lllll}2 & 72.1 & 72.4 & 72.7 & 72.7 \\ 3 & 73.2 & 72.8 & 72.9 & 72.9\end{array}$

$\begin{array}{lllll}4 & 72.3 & 72.3 & 72.4 & 72.4\end{array}$

$\begin{array}{lllll}5 & 72.8 & 72.9 & 73.5 & 73.5\end{array}$

$\begin{array}{lllll}6 & 72.2 & 71.9 & 71.9 & 71.9 \\ 7 & 73.0 & 72.9 & 72.8 & 72.9\end{array}$

$\begin{array}{lllll}8 & 71.1 & 71.1 & 71.2 & 71.2\end{array}$

$968.4 \quad 68.4 \quad 68.8 \quad-$

$\begin{array}{llllll}10 & 68.3 & 69.0 & 69.4 & 69.5 & 33.64\end{array}$

$\begin{array}{lllllll}11 & 69.1 & 69.3 & 69.6 & 69.6 & 34.13 & -\end{array}$

$\begin{array}{llllll}12 & 68.1 & 68.2 & 69.8 & 70.0 & 33.54\end{array}$

$\begin{array}{llllll}13 & 67.5 & 67.6 & 67.8 & - & 33.51\end{array}$

$\begin{array}{lllllll}14 & 68.0 & 68.0 & 67.9 & 68.0 & 33.70 & 33.76\end{array}$

$\begin{array}{lllllll}15 & 68.6 & 68.6 & 68.7 & 69.0 & 33.76 & - \\ 16 & 69.7 & 69.2 & 69.3 & 69.3 & 34.05 & -\end{array}$

$\begin{array}{lllllll}17 & 67.9 & 68.8 & 69.0 & 69.0 & 34.50 & =\end{array}$

$\begin{array}{llllll}18 & 68 . & - & - & - & 33.10 \\ 19 & 68.2 & 68.3 & 68.3 & 68.3 & 34.01\end{array}$

$\begin{array}{lllllll}20 & 68.7 & 68.6 & 68.7 & 68.7 & 33.92 & -\end{array}$

$21 \quad 68.9 \quad 68.8 \quad 68.8 \quad 68.8 \quad 34.54 \quad 34.60$

$2269 .-34.62-$

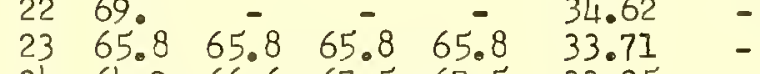

$\begin{array}{lllllll}24 & 64.9 & 66.6 & 67.5 & 67.5 & 33.25 & -\end{array}$

$\begin{array}{lllllll}25 & 62.8 & 62.8 & 64.3 & 65.1 & 32.56 & -\end{array}$

$\begin{array}{llllll}26 & 63.4 & 67.2 & 67.3 & 67.5 & 33.30\end{array}$

$\begin{array}{lllllll}27 & 67.1 & 67.2 & 67.2 & 67.2 & 35.11 & -\end{array}$

$\begin{array}{lllllll}28 & 63.1 & 63.2 & 63.2 & 64.9 & 33.36 & 33.90\end{array}$

$2964.1 \quad 64.1 \quad 64.1 \quad 64.1 \quad 34.24 \quad-$

$\begin{array}{lllllll}30 & 63.8 & 63.6 & 63.5 & 63.6 & 34.41 & -\end{array}$

$\begin{array}{lllllll}\text { Mean } & 68.4 & 68.6 & 68.9 & 69.1 & 33.98 & 34.20\end{array}$
December

Temperature of $\quad$ Salintiy $\%$

$0^{\prime} \quad 30^{\prime} 50^{\prime} 70^{\prime} 0^{\prime} 70^{\prime}$

$\begin{array}{llllll}63.8 & 63.8 & 63.8 & 53.8 & 34.41 & -\end{array}$

$\begin{array}{lllll}63.8 & 63.8 & 63.8 & 63.8 & 34.43\end{array}$

$64.264 .2 \quad 64.2 \quad 64.2 \quad 35.10=$

$67.167 .1 \quad 67.1 \quad 67.1 \quad 36.01 \quad$

$\begin{array}{llllll}66.0 & 66.4 & 66.6 & 66.4 & 35.89 & 36.14\end{array}$

$\begin{array}{lllll}63.0 & 66.4 & 66.6 & 66.5 & 35.02\end{array}$

$\begin{array}{llllll}62.5 & 66.0 & 66.0 & 66.0 & 34.60\end{array}$

$72.871 .570 .6-36.28$

$\begin{array}{lllll}73.4 & 73.4 & 73.4 & 73.4 & 36.27\end{array}$

$\begin{array}{llllll}68.0 & 68.0 & 67.5 & 67.1 & .27 & -\end{array}$

$\begin{array}{llllll}70.5 & 70.1 & 68.6 & 68.5 & .10 & -\end{array}$

$\begin{array}{llllll}69.1 & 69.0 & 69.0 & 69.0 & .21 & 36.28\end{array}$

$\begin{array}{llllll}71.2 & 71.2 & 71.2 & 71.2 & .39 & -\end{array}$

$\begin{array}{llllll}68.0 & 68.0 & 68.0 & 67.9 & .31 & -\end{array}$

$\begin{array}{llllll}69.6 & 69.6 & 69.4 & 69.4 & .30 & -\end{array}$

$\begin{array}{llllll}70.5 & 70.5 & 70.5 & 70.5 & .36 & -\end{array}$

$\begin{array}{llllll}72.9 & 72.9 & 72.9 & 72.9 & .34 & -\end{array}$

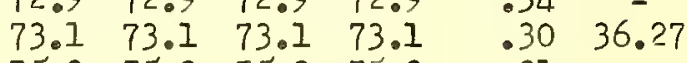

$\begin{array}{llllll}75.2 & 75.2 & 75.2 & 75.2 & .21 & -\end{array}$

$\begin{array}{llllll}74.8 & 74.8 & 74.8 & 74.8 & .25 & -\end{array}$

$\begin{array}{llllll}73.2 & 73.2 & 73.2 & 73.2 & .22 & -\end{array}$

$\begin{array}{llllll}72.2 & 72.1 & 72.0 & 71.8 & .35 & - \\ 71.5 & 71.5 & 71.5 & 71.5 & .28 & -\end{array}$

72. - - $-36.31-$

$\begin{array}{llllll}63.8 & 64.2 & 65.2 & 65.2 & 35.05 & 35.27\end{array}$

$\begin{array}{llllll}64.6 & 64.8 & 64.8 & 64.8 & 35.72 & -\end{array}$

$\begin{array}{llllll}65.8 & 65.8 & 65.8 & 65.8 & 36.18 & -\end{array}$

$63.0 .032=$

$\begin{array}{llllll}62.4 & 62.4 & 62.4 & 62.4 & 36.38 & 0 .\end{array}$

63.163 .163 .163 .1 - -

$\begin{array}{llllll}68.4 & 68.7 & 68.6 & 68.2 & 35.94 & 35.99\end{array}$ 


\section{Savannah Ii chtship}

Surface water temperatures at Savannah Iightship exhibited little departure from the mean for the period of record except for a warm March, slow April, cold last two-thirds of November and warm December.

Minimum temperatures of approximately $48^{\circ} \mathrm{F}$ apparently occurred in January (Observations did not start at this station until 18 January) and reached a maximum at the surface of less than $85^{\circ} \mathrm{F}$ in mid-August, and a maximum at the bottom of $84^{\circ} \mathrm{F}$ at the same time. The water was nearly isothemal at this shallow station throughout the year, seldoin having a gradient, surface to bottom ( 48 feet) of greater than $2^{\circ}$ s. Fxcoptions occurred in May when the surfece warmed up temporarily ahead of the underlying water.

The surface salinity was depressed, March - June to anproximately $34 \%$, increased to $36 \%$ in July, gradually leclined to $35 \%$ at year end. Bottom salinj.ty was slightly greater than the surface salinity during the first half of the year, but was essentially identical during the second half.
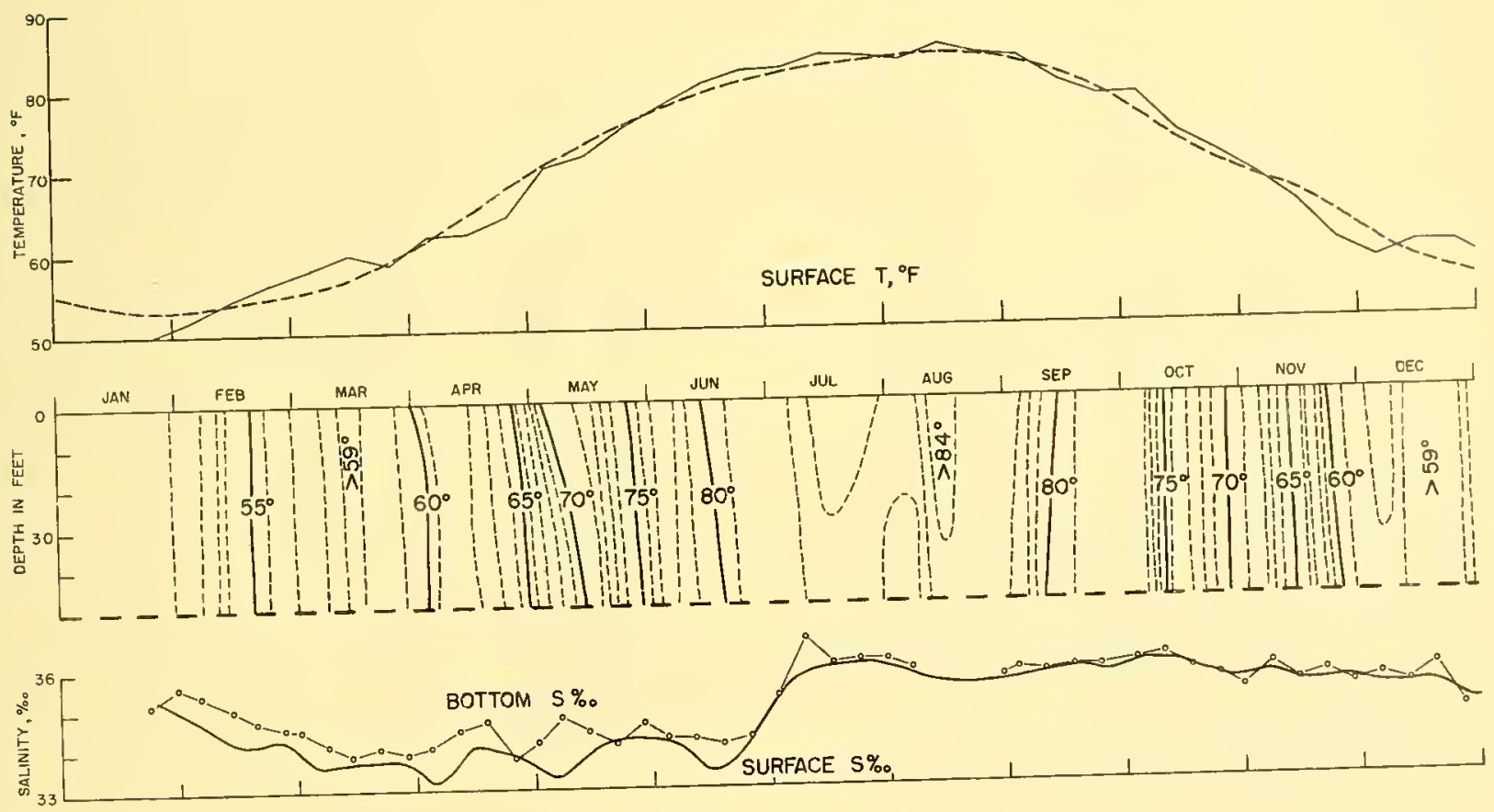

Figure 18. Savannah Iightshio, 1956. (Dashed line in upper diagram, mean for period $1947-1955)$ 
January

Tulosture ${ }_{T}$ Salinity $\%$

Denth $\quad$ Day $\quad 30: 48: 0148$

$\begin{array}{llllll}1 & - & - & - & - & - \\ 2 & - & - & - & - & - \\ 3 & - & - & - & - & - \\ 4 & - & - & - & - & - \\ 5 & - & - & - & - & - \\ 6 & - & - & - & - & - \\ 7 & - & - & - & - & - \\ 8 & - & - & - & - & - \\ 9 & - & - & - & - & - \\ 70 & - & - & - & - & -\end{array}$

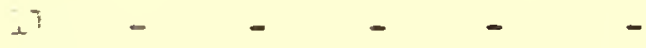

12 - - - - -

13 - - - -5

I5 - - - - - -5

16 - $-\quad=-0$

$\begin{array}{llllll}18 & 47.7 & 49.0 & 49.0 & 34.05 & 34.74\end{array}$

$\begin{array}{llllll}19 & 48.8 & 48.9 & 48.8 & .57 & -\end{array}$

$26 \quad 9.0 \quad-\quad=.08$ -

$\begin{array}{cccccc}21 & 49.0 & - & - & .72 & - \\ 22 & 48.7 & 48.7 & 48.6 & .40 & - \\ 22 & 49.6 & 49.4 & 49.2 & 34.99 & 35.20 \\ 24 & 50.0 & 50.1 & 50.1 & 35.41 & - \\ 25 & 49.3 & 50.8 & 51.3 & .31 & - \\ 26 & 50.1 & 50.1 & 50.1 & .51 & - \\ 27 & 50.3 & 50.3 & 50.8 & .50 & - \\ 28 & 51.1 & 51.2 & 51.2 & .87 & - \\ 29 & 40.4 & 49.5 & 49.7 & .21 & - \\ 30 & 51.1 & 50.8 & 50.9 & .69 & 35.64 \\ 31 & 51.3 & 51.5 & 51.7 & .69 & -\end{array}$

Mean

\section{February}

Temperature ${ }^{\circ}$ F Salinity $\%$

$\begin{array}{lllll}0^{\prime} & 30^{\prime} & 48^{\prime} & 0^{\prime} & 48^{\prime}\end{array}$

$\begin{array}{lllll}50.9 & 50.9 & 50.8 & 35.36 & -\end{array}$

$\begin{array}{lllll}51.0 & 50.8 & 50.8 & .33 & -\end{array}$

51.0 兵.051.0 .10 -

$51.0 \quad 51.351 .5 \quad .07 \quad-$

$51.851 .5 \quad 51.2 \quad 35.72=$

兵.8 51.8 51.5 $34.95 \quad 35.39$

$\begin{array}{lllll}52.0 & 52.0 & 51.9 & 33.49 & -\end{array}$

$\begin{array}{lllll}53.6 & 52.3 & 52.5 & 33.12 & -\end{array}$

$\begin{array}{lllll}52.8 & 52.6 & 52.5 & 34.93 & -\end{array}$

$\begin{array}{lllll}52.8 & 52.6 & 52.7 & .85 & \text { - }\end{array}$

$\begin{array}{lllll}52.8 & 52.7 & 52.5 & .77 & -\end{array}$

$\begin{array}{lllll}53.0 & 52.7 & 53.7 & .38 & -\end{array}$

$\begin{array}{lllll}53.0 & 53.0 & 53.5 & 34.48 & - \\ 54.0 & 54.0 & 53.7 & 33.83 & 35.04\end{array}$

$54.054 .0 \quad 54.7 \quad 34.61$ -

$\begin{array}{lllll}54.7 & 54.7 & 54.7 & 33.32 & -\end{array}$

$\begin{array}{lllll}53.9 & 53.9 & 54.0 & 34.49 & -\end{array}$

$\begin{array}{lllll}54.5 & 53.8 & 53.7 & 34.39 & -\end{array}$

$56.0 \quad 55 . ? \quad 55.7 \quad 33.58$ -

$\begin{array}{lllll}56.9 & 56.1 & 55.7 & 33.91 & 34.72\end{array}$

$55.5 \quad 55.5 \quad 55.8 \quad 34.42=$

$55.0 \quad 54.7 \quad 55.0 \quad 33.76$ -

$\begin{array}{llll}55.4 & 55.2 & 55.2 & 34.21\end{array}$

$55.254 .9 \quad 55.1 \quad .11_{1}-$

$\begin{array}{lllll}56.0 & 56.0 & 55.0 & .20 & -\end{array}$

$\begin{array}{lllll}56.7 & 56.7 & 56.7 & .36 & - \\ 56.8 & 56.8 & 56.8 & .55 & 34.55\end{array}$

$\begin{array}{llll}57.7 & 57.5 & 57.6 & .52\end{array}$

$\begin{array}{lllll}57.4 & 57.4 & 57.4 & .56 & -\end{array}$

$54.0 \quad 53.9 \quad 53.8 \quad 34.42 \quad 34.92$ 
SAVANNAH LIGHTSHIP

$31^{\circ} 56.5 \mathrm{~N} .80^{\circ} 39.6 \mathrm{w} . \quad$ Depth of water -72 feet

1956

March

April

Temperature oF Salinity $\%$

Temperature $O_{F} \quad$ Salinity $\%$

Depth

Day $0^{\prime} \quad 30^{\prime} \quad 48^{\prime} 0^{\prime} \quad 48^{\circ}$

$1 \quad 56.2 \quad 56.4 \quad 56.4 \quad 34.44 \quad 34.49$

$257.0 \quad 57.0 \quad 57.0 \quad 34.28$ -

$357.0-33.25-$

$\begin{array}{llllll}4 & 57.3 & 57.4 & 57.5 & 33.58 & -\end{array}$

$\begin{array}{llllll}5 & 57.5 & 57.5 & 57.5 & .33 & -\end{array}$

$\begin{array}{lllll}6 & 58.0 & 57.4 & 57.4 & 33.47\end{array}$

$\begin{array}{llllll}7 & 59.5 & 57.8 & 57.5 & 32.44 & - \\ 8 & 58.8 & 58.5 & 57.8 & 33.64 & 34.12\end{array}$

$\begin{array}{lllllll}9 & 58.0 & 58.1 & 58.1 & 33.79 & \text { - }\end{array}$

$\begin{array}{lllll}10 & 53.3 & 58.3 & 58.3 & 33.96\end{array}$

$\begin{array}{llllll}11 & 58.5 & 58.3 & 58.3 & 33.75 & \text { - }\end{array}$

$\begin{array}{llllll}12 & 59.0 & 59.0 & 59.0 & .91 & -\end{array}$

$\begin{array}{cccccc}13 & 59.0 & 59.0 & 59.0 & .84 & - \\ 14 & 59.8 & - & - & .85 & 33.87\end{array}$

$\begin{array}{llllll}144 & 59.8 & - & - & .85 & 33.87 \\ 15 & 61.0 & 60.3 & 60.3 & .29 & -\end{array}$

$\begin{array}{lllll}16 & 60.5 & 60.5 & 60.5 & .74\end{array}$

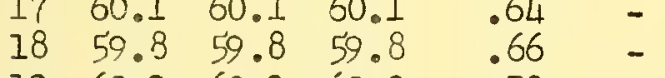

$\begin{array}{lllll}19 & 60.2 & 60.2 & 60.2 & .72\end{array}$

$\begin{array}{llllll}20 & 59.1 & 59.1 & 59.1 & .69 & -\end{array}$

$21 \quad 58.3 \quad 58.3 \quad 58.0 \quad 34.07 \quad 34.06$

2258.0 - -34.10 -

$2358.058 .058 .0 \quad 34.10=$

$\begin{array}{lllll}24 & 58.2 \quad 58.2 & 58.2 & 33.98 & -\end{array}$

$\begin{array}{lllll}25 & 58.0 \quad 58.0 \quad 57.9 \quad 34.10 & \end{array}$

$2658.0 \quad 38.0 \quad 58.0 \quad 33.76$ -

$\begin{array}{llllll}27 & 58.0 & 58.0 & 58.0 & 33.91 & - \\ 28 & 58.5 & 58.3 & 58.3 & 33.11 & 33.90\end{array}$

$2958.5 \quad-\quad-\quad .76$ -

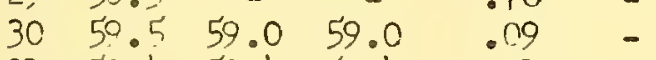

$3159.4 \quad 59.4 \quad 59.4 \quad .31$ -

$0^{\prime} \quad 30^{\prime} \quad 48^{\prime} 0^{\prime} \quad 481$

$\begin{array}{lllll}59.7 & 59.6 & 59.5 & 33.71 & -\end{array}$

$\begin{array}{llll}60.8 & 60.1 & 60.2 & 33.50\end{array}$

$\begin{array}{lllll}62.5 & 59.6 & 59.5 & 32.87\end{array}$

$\begin{array}{rrrrr}62.0 & 60.6 & 60.4 & .20 & 34.07 \\ 62.5 & 60.5 & 60.5 & .66 & \text { - }\end{array}$

$\begin{array}{lllll}63.8 & 60.9 & 60.9 & .02 & -\end{array}$

$\begin{array}{llll}62.8 & 61.3 & 61.2 & 32.23\end{array}$

$\begin{array}{llll}61.8 & 62.1 & 62.3 & 33.94\end{array}$

$61.7 \quad 61.7 \quad 61.7 \quad 34.51$

$\begin{array}{llll}61.6 & 61.6 & 61.6 & .33\end{array}=$

$\begin{array}{lllll}61.5 & - & - & .39 & 34.48\end{array}$

$60.9 \quad 60.8 \quad 60.8 \quad .49 \quad$.

$61.5 \quad 61.1 \quad 61.4 \quad .52$

$\begin{array}{llll}61.6 & 61.5 & 61.5 & .54\end{array}$

$\begin{array}{llll}62.0 & 62.1 & 62.2 & .68\end{array}$

$\begin{array}{llll}61.8 & 61.8 & 61.9 & 34.49\end{array}$

$\begin{array}{lllll}62.1 & 62.2 & 62.5 & 33.81 & -\end{array}$

$\begin{array}{lllll}62.3 & 63.1 & 63.1 & 31.97 & 34.70\end{array}$

$\begin{array}{lllll}63.5 & 62.9 & 63.0 & 34.14 & -\end{array}$

$62.8 \quad 63.0 \quad 63.0 \quad 33.52$

$62.762 .7-34.11-$

$\begin{array}{lllllll}62.9 & 62.8 & 62.8 & - & -\end{array}$

$64.0 \quad-50.53 .05=$

$\begin{array}{rrrrr}63.7 & 63.5 & 63.5 & .43 & - \\ 63.8 & 63.8 & 63.8 & 83.72 & 33.74\end{array}$

$63.5 \quad 63.5 \quad 63.4 \quad 34.48$

$64.164 .0 \quad 64.0 \quad 34.48$

$64.9 \quad 64.264 .2 \quad 33.92$ -

$65.864 .2 \quad-\quad .67$ -

$\begin{array}{lllll}66.0 & 65.1 & 65.1 & 33.53 & .5\end{array}$

$\begin{array}{llllll}\text { Mean } & 58.6 \quad 58.5 & 58.5 & 33.73 & 34.09\end{array}$

$\begin{array}{lllll}62.7 & 62.2 & 62.1 & 33.69 & 34.25\end{array}$ 


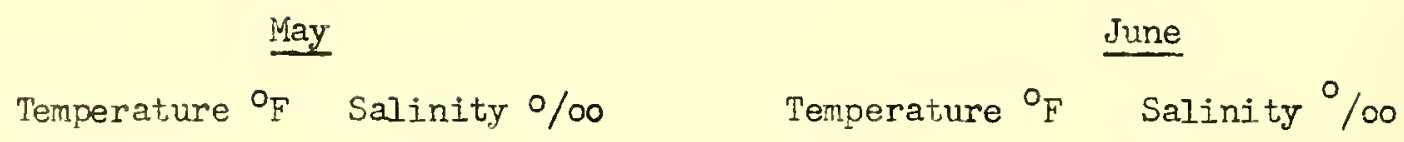

Depth

Day $0^{\prime} \quad 30^{\prime} 48^{\prime} 0^{\prime} 48^{\prime}$

$\begin{array}{llllll}1 & 69.1 & 66.3 & 66.2 & 32.59 & 34.15\end{array}$

$272.7-31.88$ -

$\begin{array}{llllll}3 & 70.2 & 66.9 & 67.0 & 33.04 & -\end{array}$

$471 .-32.73-$

$\begin{array}{llllll}5 & 71.9 & 67.1 & 67.1 & .58 & -\end{array}$

$\begin{array}{llllll}6 & 70.9 & 57.3 & 67.2 & 32.84 & - \\ 7 & 72.4 & 67.4 & 67.4 & 33.04 & 34.78\end{array}$

$\begin{array}{llllll}8 & 58.1 & 68.1 & 68.1 & 34.42 & -\end{array}$

$\begin{array}{llllll}9 & 67.2 & 67.2 & 67.1 & .84 & -\end{array}$

$\begin{array}{lllll}10 & 68.0 & 67.5 & 67.5 & 31.96\end{array}$

$\begin{array}{llllll}11 & 68.3 & 68.0 & 67.9 & 35.02 & -\end{array}$

$\begin{array}{llllll}12 & 69.1 & 68.7 & 68.6 & 34.94 & -\end{array}$

$\begin{array}{llllll}13 & 71.1 & 69.7 & 69.6 & 34.20 & - \\ 11 & 70.8 & 70.1 & 70.1 & 33.70 & 34.52\end{array}$

$\begin{array}{llllll}15 & 73.2 & 70.9 & 70.9 & 32.90 & -\end{array}$

$\begin{array}{llllll}16 & 73.1 & 71.7 & 71.3 & 33.71 & -\end{array}$

$\begin{array}{llllll}17 & 72.4 & 72.3 & 71.9 & .81 & -\end{array}$

$\begin{array}{llllll}10 & 72.1 & 72.0 & 71.9 & .49 & -\end{array}$

$\begin{array}{llllll}19 & - & - & - & - & -\end{array}$

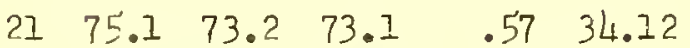

$\begin{array}{lllllll}22 & 74.1 & 73.1 & 73.1 & .94 & -\end{array}$

$\begin{array}{llllll}23 & 74.8 & 74.4 & 7 ! .5 & .96 & -\end{array}$

$\begin{array}{llllll}24 & 76.3 & 75.5 & 75.3 & 33.79 & -\end{array}$

$\begin{array}{llllll}25 & 75.1 & 75.9 & 75.9 & 34.85 & -\end{array}$

$\begin{array}{lllll}26 & 74.0 & 73.9 & 73.8 & .76\end{array}$

$\begin{array}{lllllc}27 & 73.9 & 78.7 & 73.6 & .79 & - \\ 28 & 75.4 & 74.0 & 74.0 & .48 & 34.62\end{array}$

$\begin{array}{lllllll}29 & 75.2 & 74.1 & 74.1 & .07 & -\end{array}$

$\begin{array}{llllll}30 & 74.5 & 73.9 & 73.7 & 34.40 & -\end{array}$

$\begin{array}{llllll}31 & 76.8 & 74.1 & 74.1 & 33.85 & -\end{array}$

$\begin{array}{llllll}\text { Mean } & 72.4 & 73.0 & 70.9 & 33.83 & 34.44\end{array}$ $0^{\prime} \quad 30^{\prime} 48^{\prime} 0^{\prime} \cdot 48^{\prime}$

$\begin{array}{llll}77.3 & 76.9 & 76.8 & 34.14\end{array}$

$\begin{array}{llll}77.8 & 77.5 & 77.4 & 33.68\end{array}$

$\begin{array}{llll}77.7 & 77.2 & 77.3 & 33.76\end{array}$

$\begin{array}{lllll}78.0 & 77.6 & 77.4 & 34.21 & 34.24\end{array}$

$\begin{array}{llllll}77.5 & 77.7 & 77.3 & .21 & -\end{array}$

77.577 .477 .0 -

$\begin{array}{llll}77.2 & 77.0 & 76.9 & .26\end{array}$

$\begin{array}{llll}77.5 & 77.0 & 77.0 & .42\end{array}$

$\begin{array}{llll}78.0 & 77.3 & 77.1 & 34.37\end{array}$

$\begin{array}{lllll}78.0 & 78.0 & 77.9 & 33.84\end{array}$

$78.7 \quad 78.0 \quad 78.0 \quad 31.14 \quad 34.21$

$\begin{array}{lllll}78.8 & 78.1 & 78.7 & 34.20 & =\end{array}$

$80.0 \quad 78.9 \quad 78.7 \quad 33.34 \quad-$

$\begin{array}{lllll}80.1 & 79.2 & 79.1 & .36 & -\end{array}$

$\begin{array}{llll}80.2 & 79.3 & 79.2 & .12\end{array}$

$\begin{array}{lllll}80.7 & 79.6 & 79.5 & .06 & =\end{array}$

$\begin{array}{lllll}81.3 & 79.7 & 79.6 & 33.08 & -\end{array}$

$\begin{array}{lllll}82.5 & 80.0 & 80.0 & 32.53 & 34.08\end{array}$

$81.0 \quad 80.8 \quad 80.6 \quad 33.42 \quad-$

$80.4 \quad 80.4 \quad 80.4 \quad 33.75$ -

$80.5 \quad 80.2 \quad 80.0 \quad 34.03=$

82.0 - -33.79 -

$81.4-33.82=$

$82.0 \quad 81.7 \quad 81.7 \quad 34.03 \quad-$

$\begin{array}{lllll}81.6 & 81.4 & 81.4 & 34.23 & 34.25\end{array}$

$82.0 \quad 81.6 \quad 81.6 \quad 33.82 \quad$ -

$81.7 \quad 81.6 \quad 81.5 \quad 34.22 \quad-$

$81.5 \quad 81.4 \quad 81.4 \quad: 52=$

$82.0 \quad 81.5 \quad 81.5 \quad .98 \quad-$

$82.3 \quad 82.0 \quad 82.0 \quad 34.60$ -

$\begin{array}{lllll}79.9 & 79.2 & 79.2 & 33.89 & 34.20\end{array}$ 


\section{July}

Temperature OF Salinity $\%$

Decth $\begin{array}{lllll}1 & 80.1 & 80.1 & 80.1 & 34.80\end{array}$

$\begin{array}{llllll}2 & 82.0 & 81.1 & 81.1 & 35.18 & 35.23\end{array}$

$\begin{array}{llllll}3 & 81.5 & 81.4 & 81.1 & 35.49 & -\end{array}$

$\begin{array}{lllll}4 & 81.2 & 81.5 & 81.5 & 34.86\end{array}$

$\begin{array}{llllll}5 & 82.1 & 81.7 & 81.6 & 35.17 & -\end{array}$

$\begin{array}{llllll}6 & 82.1 & 81.8 & 81.6 & .39 & -\end{array}$

$\begin{array}{llllll}7 & 82.1 & 82.0 & 81.8 & 35.99 & -\end{array}$

$\begin{array}{llllll}8 & 82.6 & 82.4 & 82.2 & 36.16\end{array}$

$\begin{array}{llllll}9 & 82.5 & 82.5 & 82.3 & .24 & 36.64\end{array}$

$\begin{array}{llllll}10 & 82.4 & 82.3 & 82.1 & 36.24 & \text { - }\end{array}$

$\begin{array}{lllll}11 & 82.3 & 82.1 & 82.1 & 35.78\end{array}$

$\begin{array}{llllll}12 & 83.0 & 82.7 & 82.5 & 36.01 & -\end{array}$

$\begin{array}{llllll}13 & 83.1 & 82.7 & 82.5 & 36.08 & -\end{array}$

$\begin{array}{llllll}14 & 83.1 & 82.9 & 82.6 & 35.82 & -\end{array}$

$\begin{array}{llllll}15 & 83.7 & 83.3 & 83.2 & .82 & -\end{array}$

$\begin{array}{llllll}16 & 83.2 & 83.0 & 83.0 & .25 & 35.98\end{array}$

I7 $84.0 \quad 83.4 \quad 82.9 \quad .91 \quad$ -

$\begin{array}{llllll}18 & 85.2 & 82.7 & 82.6 & 35.82 & -\end{array}$

$\begin{array}{llllll}19 & 83.9 & 83.5 & 82.8 & 36.16 & -\end{array}$

$\begin{array}{llllll}20 & 83.6 & 83.3 & 83.2 & 36.19 & -\end{array}$

$\begin{array}{llllll}21 & 83.3 & 83.7 & 83.4 & 35.83 & -\end{array}$

$\begin{array}{llllll}2.2 & 84.3 & 83.8 & 83.5 & 35.71 & -\end{array}$

$\begin{array}{llllll}23 & 84.7 & 83.6 & 83.4 & 36.08 & 36.07\end{array}$

$\begin{array}{llllll}24 & 85.4 & 84.3 & 83.3 & .07 & -\end{array}$

$\begin{array}{llllll}25 & 84.0 & 83.2 & 83.1 & .14 & =\end{array}$

$\begin{array}{llllll}26 & 83.5 & 82.9 & 82.9 & 36.06 & -\end{array}$

$\begin{array}{llllll}27 & 83.9 & 82.5 & 82.0 & 35.90 & -\end{array}$

$\begin{array}{lllll}28 & 82.8 & 82.1 & 81.5 & 36.00\end{array}$

$\begin{array}{llllll}29 & 82.2 & 81.6 & 80.3 & 35.96 & -\end{array}$

$\begin{array}{llll}30 & 80.7 \quad 80.0-36.00 & 36.08\end{array}$

$\begin{array}{llllll}31 & 82.0 & 81.5 & 80.2 & 35.95 & -\end{array}$

$\begin{array}{llllll}\text { Mean } & 82.9 & 82.5 & 82.2 & 35.81 & 36.00\end{array}$
August

Temperature ${ }^{\circ}$ F Salinity $\%$

$0^{\prime} \quad 30^{\prime} \quad 4^{\prime} 0^{\prime} \quad 48^{\circ}$

82.C $\quad 81.2 \quad 81 . \mathrm{C} \quad 36.08$

$83.5 \quad 81.4 \quad 81.0 \quad 35.22$

$\begin{array}{llll}82.9 & 81.6 & 81.3 \quad .70\end{array}$

$81.8 \quad 81.7 \quad 81.5 \quad .60$

$\begin{array}{llll}81.2 & 80.5 & 80.3 & .91\end{array}$

$\begin{array}{lllll}82.7 & 81.6 & 81.3 & .67 & 35.84\end{array}$

$84.0 \quad 83.4 \quad 82.8 \quad .62 \quad-$

$83.5 \quad 83.0 \quad 82.9 \quad .77=$

$\begin{array}{llll}82.8 & 82.3 & 82.3 & .72\end{array}$

$83.0 \quad 82.5 \quad 82.4 \quad .92 \quad-$

$\begin{array}{lllll}83.7 & 83.0 & 82.9 & .66\end{array}$

$84.1 \quad 84.0 \quad 83.8 \quad .42$

$\begin{array}{llll}34.8 & 84.3 & 83.9 & .34\end{array}$

$84.5 \quad 84.0 \quad 83.8 \quad .45$

$85.0 \quad 84.1 \quad 83.9 \quad 0.52$

$\begin{array}{llll}34.2 & 84.1 & 84.2 & .69\end{array}$

$\begin{array}{llll}84.6 & 84.3 & 84.2 & .60\end{array}$

$84.9 \quad 84.3 \quad 84.0 \quad 35.53$ -

$84.9 \quad 84.7 \quad 84.3 \quad 34.71$ -

$\begin{array}{llll}85.2 & 84.3 \quad 84.1 \quad 35.42 & -\end{array}$

$\begin{array}{llll}84.1 & 84.1 & 83.9 & .82\end{array}$

$83.4 \quad 83.3 \quad 83.3 \quad .74 \quad-$

$83.0 \quad 83.0 \quad 83.0 \quad .82$

$83.5 \quad 83.1 \quad 83.0 \quad .82=$

$\begin{array}{llll}83.8 & 83.3 & 83.2 & .66\end{array}$

$\begin{array}{llll}83.5 & 83.2 & 83.2 & .44\end{array}$

$\begin{array}{llll}84 . \mathrm{C} & 83.7 & 83.7 & .52\end{array}$

$83.2 \quad 83.0 \quad 83.0 \quad .47 \quad-$

$\begin{array}{lllll}83.0 & 83.0 & 82.9 & 35.30 & 35.62\end{array}$

$\begin{array}{llllll}82.7 & 82.7 & 82.7 & 33.81 & \text { - }\end{array}$

$\begin{array}{lllll}83.1 & 82.8 & 82.5 & 35.38\end{array}$

$\begin{array}{llll}83.6 & 83.1 & 82.9 & 35.52\end{array}$ 


\section{Septumber}

Temperature 0 Sating $\%$
Qctober

Temperature os Salinity $\%$

$0^{\circ} \quad 30^{\circ} \quad 48^{\circ} \quad 0^{\circ} \quad 49^{\circ}$

$\begin{array}{ccccc}77.2 & 77.1 & 77.2 & 35.87=\end{array}$

$\begin{array}{llll}77.9 & 77.9 & 77.9 & 35.90\end{array}$

$\begin{array}{llll}78.2 & 78.1 & 78.1 & 36.01\end{array}$

$\begin{array}{lllll}78.5 & 78.2 & 78.0 & 35.99\end{array}$

$79.0 \quad 79.079 .0 \quad 36.00$ -

$79.079 .179 .135 .87=$

$\begin{array}{lllll}77.9 & 77.9 & 77.9 & 35.90 & \text { - }\end{array}$

$73.178 .1 \quad 72.2 \quad 36.05$

$\begin{array}{lllll}77.9 & 72.9 & 77.9 & 36.09 & 36.09\end{array}$

$\begin{array}{lllll}76.8 & 76.9 & 76.9 & 35.99 & -\end{array}$

$\begin{array}{lllll}75.5 & 75.7 & 75.7 \quad 35.77 & -\end{array}$

74.874 .874 .836 .14 -

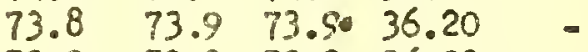

$\begin{array}{lllll}73.2 & 73.3 & 73.2 & 36.03 & =\end{array}$

$\begin{array}{llll}72.5 & 72.6 & 72.6 & 35.75\end{array}$

$\begin{array}{lllll}11.9 & 71.9 & 71.9 & 35.72 & 35.72\end{array}$

$\begin{array}{lllll}71.6 & 71.7 \quad 71.8 & 35.53 & -\end{array}$

$\begin{array}{lllll}72.0 & 71.9 & 71.9 & 35.67 & -\end{array}$

$71.9 \quad 72.0 \quad 72.0 \quad 35.67$ -

$71.771 .7 \quad 71.8 \quad 35.67 \quad-$

$\begin{array}{lllll}72.7 & 72.7 & 71.7 & 35.62 & -\end{array}$

$\begin{array}{lllll}72.1 & 71.8 & 71.8 & 35.69 & - \\ 71.5 & 71.5 & 71.5 & 35.56 & 35.57\end{array}$

$\begin{array}{lllll}71.4 & 71.3 & 71.5 & 35.47 & \end{array}$

$71.0 \quad 72.0 \quad 71.1 \quad 35.51$ -

$70.4 \quad 70.4 \quad 70.6 \quad 35.47$ -

$70.170 .170 .1 \quad 35.38$ -

$\begin{array}{cccccc}- & - & - & 35.16 & -\end{array}$

$\begin{array}{lllll}69.2 & 59.2 & 60.2 & 35.24 & 35.24\end{array}$

$74.0 \quad 74.0 \quad 74.0 \quad 35.74 \quad 35.72$ 
November

Temperature ${ }^{\circ}$ F Salinity $\%$

\section{December}

Temperature ${ }^{\circ} \mathrm{F} \quad$ Salinity $\%$

Depth

Day $0^{\prime} \quad 30^{\prime} \quad 48^{\prime} 0^{\prime} \quad 48^{\prime}$

$\begin{array}{lllllll}1 & 68.7 & 68.7 & 68.7 & 35.24 & -\end{array}$

$\begin{array}{rrrrrr}2 & 69.1 & 69.1 & 69.0 & .32 & - \\ 3 & 68.9 & 68.7 & 68.7 & .56 & -\end{array}$

$\begin{array}{llllll}4 & 68.9 & 68.8 & 68.8 & .80 & -\end{array}$

$\begin{array}{llllll}5 & 68.3 & 68.3 & 68.3 & .72 & -\end{array}$

$\begin{array}{llllll}6 & 68.2 & 68.2 & 68.1 & .77 & - \\ 7 & 68.2 & 68.1 & 68.2 & .78 & 35.76\end{array}$

$\begin{array}{llllll}8 & 67.5 & 67.9 & 67.9 & .68 & -\end{array}$

$\begin{array}{lllll}9 & 67.0 & 67.0 & 67.1 & .39\end{array}$

$1065.465 .866 .0 \quad 35.73$ =

$1763.4 \quad 63.965 .4 \quad 34.04=$

$1264 . ? 64.264 .2 \quad 35.32$ -

$\begin{array}{llllll}13 & 63.8 & 63.6 & 63.8 & .40 & -\end{array}$

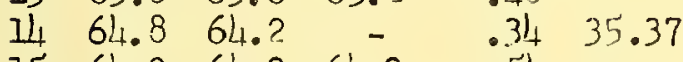

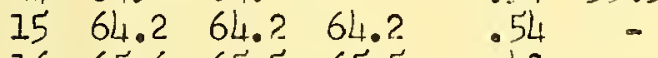

$\begin{array}{lllll}16 & 65.6 & 65.5 & 65.5 & .43\end{array}$

$\begin{array}{llllll}17 & 66.2 & 65.9 & 65.8 & .111 & - \\ 1.8 & 65.0 & 65.1 & 65.2 & .144 & -\end{array}$

$\begin{array}{lllll}19 & 64.3 & 64.5 & 64.6 & .60\end{array}$

$2063.964 .1 \quad 64.7 .58$ -

$\begin{array}{cccccc}21 & 63.7 & 63.7 & 63.6 & .60 & 35.59 \\ 22 & 63.7 & 63.8 & 63.8 & .45 & - \\ 23 & 62 . ? & 62.3 & 62.3 & .149 & - \\ 24 & 61.0 & 61.0 & 61.5 & - & - \\ 25 & 60.6 & 60.6 & 60.6 & .11 & - \\ 26 & 60.0 & 60.0 & 60.0 & .13 & - \\ 27 & 52.4 & 53.1 & 53.3 & 35.58 & - \\ 28 & 57.0 & 57.7 & - & 34.98 & 35.26 \\ 29 & 58 . ? & 58.2 & 58.2 & 35.55 & - \\ 30 & 58.4 & 58.1 & 58.4 & 35.79 & - \\ 31 & - & - & - & - & -\end{array}$

Mean 64.1 64.? 64.5 35.44 35.50

$\begin{array}{lllll}0^{\prime} & 30^{\prime} & 481 & 0^{\prime} \quad 481\end{array}$

$58.3 \quad 58.4 \quad 58.5 \quad 35.92=$

$\begin{array}{lllll}58.0 & 58.1 & 58.3 & 35.79\end{array}$

$56.257 .4 \quad 58.0 \quad 34.93=$

$\begin{array}{rrrrr}56.8 & 57.3 & 57.3 & 35.44 & - \\ 55.3 & 56.1 & 56.1 & .31 & 35.45\end{array}$

$\begin{array}{lllll}56.7 & 57.8 & 57.8 & .05 & -\end{array}$

$\begin{array}{llll}58.1 & 58.1 & 58.2 & .56\end{array}$

$\begin{array}{llll}58.2 \quad 58.2 & 58.3 & 35.32 & -\end{array}$

$\begin{array}{llll}57.7 & 58.3 & 58.3 & 33.70\end{array}$

$58.7 \quad 59.0 \quad 60.2 \quad 35.25$ -

$\begin{array}{lllll}58.1 & 58.4 & 58.5 & .37 & -\end{array}$

$\begin{array}{lllll}58.3 & 58.4 & 58.6 & .15 & 35.22\end{array}$

$\begin{array}{llllll}59.0 & 58.5 & 58 . l_{1} & .1 l_{t} & -\end{array}$

$\begin{array}{llll}59.3 & 59.2 & 59.2 \quad .02 & -\end{array}$

$59.0 \quad 58.9 \quad 58.9 \quad .02 \quad-$

$\begin{array}{llll}59.3 & 59.3 & 59.3 & .17\end{array}$

$59.4 \quad 59.4 \quad 50.14 \quad .14$ -

59.000 .21 .70

$\begin{array}{lllll}59.9 & 59.9 & 59.9 & .88 & -\end{array}$

$59.959 .960 .0 \quad 35.80$ -

$\begin{array}{llll}60.2 & 60.1 & 60.1 & 34.91\end{array}$

$60.260 .2-35.07=$

$\begin{array}{llll}60.1 & 60.1 & 60.1 & 34.89\end{array}$

$\begin{array}{lllll}60.3 & 60.2 & 60.2 & .77\end{array}$

$\begin{array}{lllll}59.3 & 59.8 & - & .64 & 34.62\end{array}$

$\begin{array}{lllll}58.3 & 58.6 & 59.3 & .15 & -\end{array}$

$\begin{array}{lllll}58.9 & 59.0 \quad 59.0 \quad .84 & =\end{array}$

$\begin{array}{lllll}58.3 \quad 58.7 \quad 58.7 \quad 34.85 & \text { - }\end{array}$

$\begin{array}{lllll}56.8 & 57.1 & 57.1 & 35.09 & -\end{array}$

$\begin{array}{llll}57.5 & 57.2 \quad 57.3 \quad 35.06\end{array}$

$\begin{array}{lllll}58.6 & 58.7 & 58.8 & 35.13 & 35.25\end{array}$ 


\section{Literature Cited}

Bumpus, D. F.

1957. Surface water temperatures along the Atlantic and Gulf coasts of the United States. U. S. Department of the Interior, Fish and Wildlife Service, Special Scientific Report--Fisheries No. 214, $153 \mathrm{pp}$.

Bumpus, D. F., and E. L. Pierce

1957. Hydrograph $y$ ard the distribution of chaetogonaths over the continent shelf off North Carolina. Papers in Marine Biology and Oceanography, Deep-Sea Research, supplement to vol. 3, pp. 92-109.

Hachey, $\mathrm{F}_{1}$. B.

1939. Surface water temperatures of the Canadian Atlantic Coast. Jourmal of the Fisheries Research Board of Canada, vol. 4, no. 15, pp. 378-391.

Longard, J. R., and R. E. Banks

1952. Wind-induced vertical movement of the water on an open coast. Transactions of the American Geophysical Union, vol. 33, no. 3, pp. 377-380.

Parn, A. E.

1933. Geographical-ecological analysis of the seasonal changes in temperature conditions in shallow water along the Atlantic Coast of the United States. Bulletin of the Bingham Oceanographic Collection, vol. 4, article 3, $90 \mathrm{pp}$.

Rathbun, R.

1887. Ocean temperatures of the eastem coast of the United States, from observations made at twenty four lighthouses and lightships. Fisheries and Fishing Industries of the United States, Appendix to Section III. Washington. 
